\title{
Die Pest in Augsburg um 1500. Die soziale Konstruktion einer Krankheit
}

\author{
Dissertation \\ zur Erlangung des philosophischen Doktorgrades \\ an der Philosophischen Fakultät der Georg-August-Universität Göttingen
}

vorgelegt von

Mariusz Horanin

aus Kołobrzeg, Polen

Göttingen 2019 


\section{Inhaltsverzeichnis}

Verzeichnis der Abbildungen und Tabellen im Text ............................................................. 5

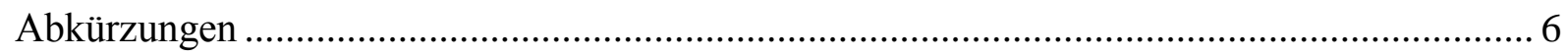

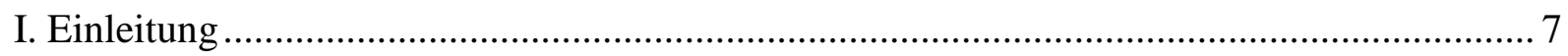

1. Das Phänomen der Pest in Gegenwart und Vergangenheit - das Problem der

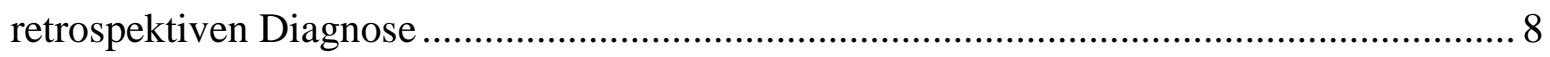

2. Die soziale Konstruktion der Pest - methodische Grundlagen, Fragestellung und

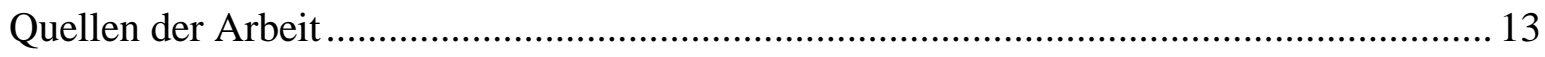

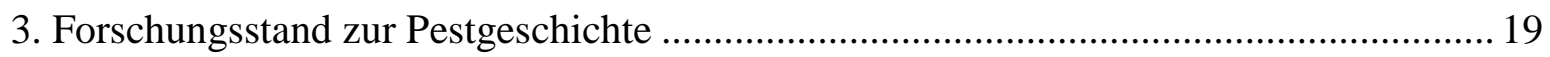

II. Das Pestgeschehen im spätmittelalterlichen Augsburg ................................................... 24

1. „Pestilentz“, „Brechen“ und „großes Sterben“- Augsburger Quellenbegriffe für die Pest

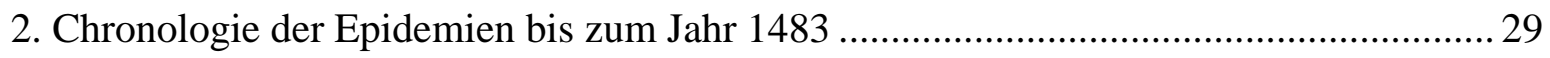

3. Grundzüge der Pestwahrnehmung und -bewältigung im Spätmittelalter........................ 37

4. Das städtische Medizinalwesen und der medizinische Diskurs in der Pestzeit ............... 39

III. Religion und Medizin: Augsburger Pestdrucke bis zur Mitte des 16. Jahrhunderts .......... 43

1. Religiöse Pestblätter: Schutzheilige in der Pestzeit .................................................... 45

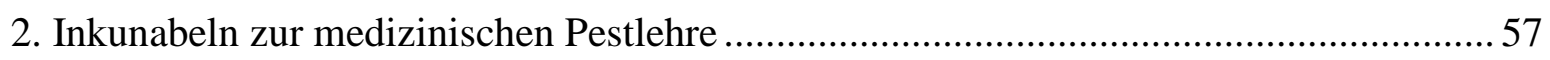

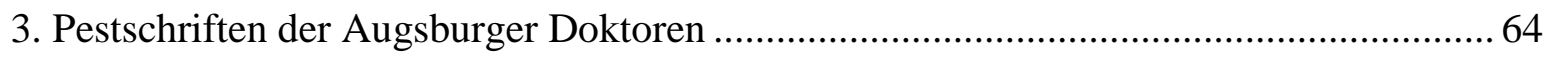

4. Augsburg als Druckort für die Pestschriften auswärtiger Autoren ............................... 70

5. Lesepublikum der medizinischen Buchdrucke ...................................................... 71

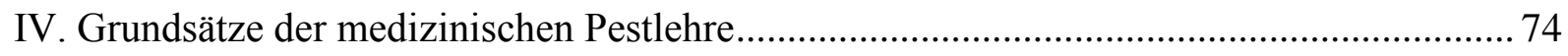

1. Das antike Medizinkonzept: Hippokrates, Galen und ihre arabischen Nachfolger ......... 75

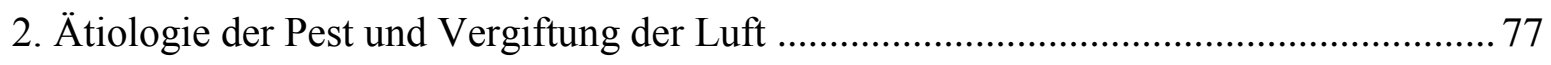

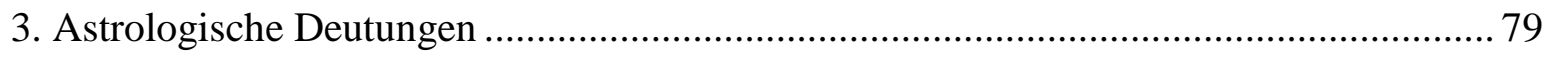

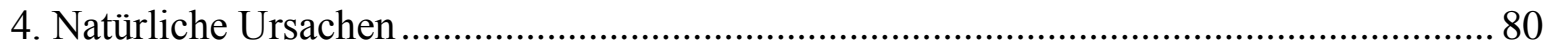




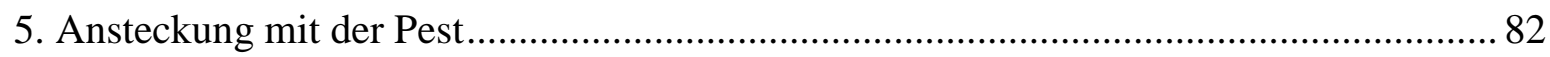

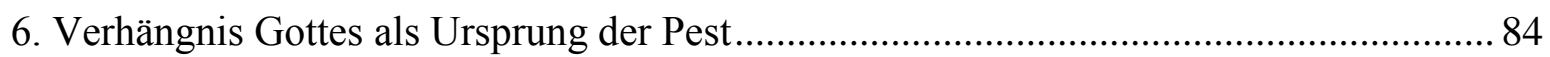

7. Heiligenverehrung in der Reformationszeit im Spiegel der Augsburger Pestschriften ... 86

V. Medizinische Anweisungen in den Pestschriften ............................................................... 95

1. Schutz vor der verdorbenen Luft............................................................................ 96

2. Ansteckungsgefahr, die von den Pestkranken ausgeht................................................... 99

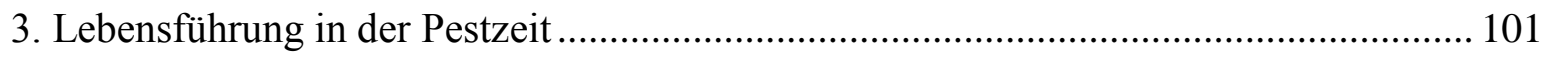

4. Prophylaktische Selbstmedikation ………………………………………………….... 104

5. Belehrungen von protestantischen Theologen zur Flucht und zum Selbstschutz vor der

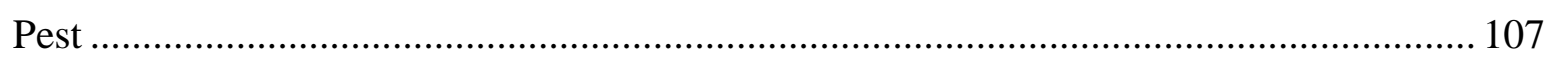

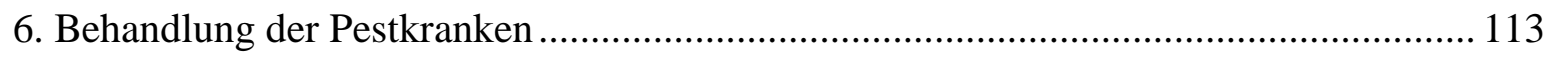

7. Wirksamkeit der medizinischen Pestlehre ……………………………….................. 123

VI. Anfänge der städtischen Pestpolitik in Augsburg zwischen 1494 und 1521 ................... 125

1. Anlegung des Unteren Gottesackers 1494/1495 .......................................................... 125

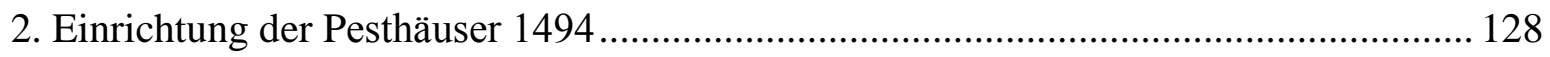

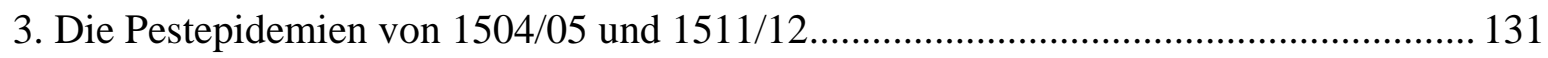

4. Ausbruch der Pest im Jahr 1521 und Anweisungen Augsburger Stadtärzte................... 134

5. Die spezielle Sitzungsordnung des Rates und die Pestflucht der Augsburger im Jahr 1521

6. Gründung des Pesthospitals (,Brechhäuser“) mit einem Friedhof im Jahr 1521 .......... 139

7. Die Ordnung für die Brechhäuser von 1521 .............................................................. 144

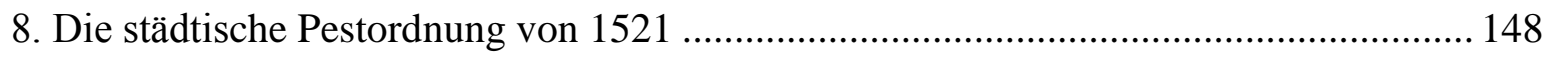

VII. Ausbau der Pestbewältigung in Augsburg bis zur Mitte des 16. Jahrhunderts............... 152

1. Anlegung des Oberen Gottesackers und Vorsorgemaßnahmen in den Jahren 1533-1534

2. Ausbruch der Epidemie und die städtische Pestordnung von 1535 ............................... 156 
3. Die Begräbnisordnung von 1535 und andere Anordnungen zur Eindämmung der Seuche 160

4. Erweiterung des städtischen Dienstpersonals in der Pestzeit 1535 162

5. Die Pestepidemie während des Augsburger Reichstages in den Jahren 1547-1548 166

6. Anordnungen zum Bettelwesen im Kontext der Seuchenbewältigung in der ersten Hälfte des 16. Jahrhunderts

VIII. Die soziale Konstruktion der Pest in Augsburg um 1500 - Einblick in den historischen Hintergrund 172

1. Aufstieg der gelehrten Mediziner zu städtischen Experten. 172

2. Beratungen der Augsburger Stadtärzte zur Regelung des städtischen Medizinalwesens

3. Kommunalisierung der Armen- und Krankenfürsorge 186

IX. Zusammenfassung 191

X. Quellen- und Literaturverzeichnis 203

1. Ungedruckte Quellen. 203

2. Gedruckte Quellen 204

3. Literatur 211 


\section{Verzeichnis der Abbildungen und Tabellen im Text}

Abb. 1: Einblattdruck, Bild des strafenden Gottes (1) [Augsburg: c. 1460-1470] .................. 46

Abb. 2: Einblattdruck, Bild des strafenden Gottes (2) [Augsburg: c. 1470-1480] .................. 48

Abb. 3: Einblattdruck, Bild der Heiligen Sebastian und Rochus,

[Augsburg oder Ulm: c. 1460-1463]

Abb. 4: Einblattdruck, Gebete zu den Heiligen Rochus und Sebastian mit ihrem Bild

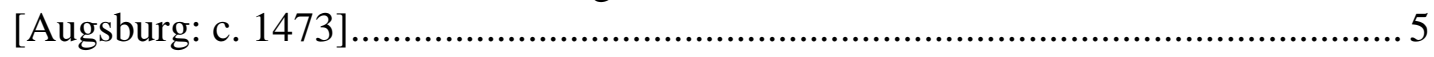

Abb. 5: Einblattdruck, Gebet zum Heiligen Sebastian mit seinem Bild (1)

[vermutlich in Augsburg: um 1475] .................................................................. 52

Abb. 6: Einblattdruck, Gebet zum Heiligen Sebastian mit seinem Bild (2)

[Augsburg, Hans Froschauer: c. 1500] ................................................................... 53

Abb. 7: Einblattdruck für die Pestzeit, [Elsass, um 1500] ....................................................56

Abb. 8: Tormanita, Vil menschen weren der pestilentz frey (1)

[Einbl. Augsburg: Günther Zainer um 1472] .............................................................5

Abb. 9: Tormanita, Vil menschen weren der pestilentz frey (2)

[Einbl. Augsburg: Günther Zainer um 1472]

Abb. 10: Titelblatt in: Jung Ambrosius, Tractatulus perutilis de pestilentia

[Augsburg: Hans Schönsberger, 20. November 1494].

Abb. 11: Abb. 11: Darstellung der tödlichen und heilbaren Apostemata und Blattern

bei einem Pestkranken, in: Böham, Ain nutzliche/

vnnd für den gemainen Man/ genugsam gegründte vnderricht

[Augsburg: gedruckt von Philipp Ulhart und zum Neudruck

aufgelegt von Johannes Vogt dem Jüngeren um 1570;

bearbeitet und ergänzt von Wolfgang Talhauser um 1547]

Abb. 12: Ardea, Ain gut und vast nutzliches Regiment [Einbl. Augsburg: 1536] ............... 91

Abb. 13: Holzschnitt über die Auswirkungen der Pest in: Lobera de Avila, Vanquete de nobles cavalleros...[Augsburg 1530]

Abb. 14: Holzschnitt über die Auswirkungen der Pest in: Petrarca,

Das Glückbuch/ Beydes des Gutten und Boesen [Augsburg 1539]

Abb. 15: Aderlassmännchen für die Pestzeit, Titelblatt in: Anonymus, Ain regiment wider Die Pestilentz [Augsburg: Johannes Schönsberger um 1525]

Abb. 16: Aderlassmännchen für die Pestzeit, Titelblatt in: Veringer,

Ein kurtz nützlichs Regiment [Augsburg: Heinrich Steiner 1533]

Abb. 17: Augsburger Stadtplan um 1550, in: Sebastian Münster, Cosmographia, ca. 1550, Städtische Kunstsammlungen Augsburg

Abb. 18: Bevölkerungstafel (1510-1583), SSBA, $2^{\circ}$ Lw Einblattdrucke nach 1500, Nr. 347 (1511-1584)

Abb. 19: Der Ausschnitt mit der Tabelle aus der Augsburger Bevölkerungstafel (1501-1750) in: Fischer, Gesundheitswesen, Bd. I, S. 67, Abb. 6 
Abb. 20: Augsburger Stadtplan in der ersten Hälfte des 18. Jahrhunderts, in:

Stetten, Geschichte der Heil. Röm. Reichs Freyen Stadt Augsburg, Frankfurt/Leipzig 1743

Abb. 21: Die Pesthospitalanlage in Augsburg (Kupferstich von Raphael Custos) aus dem Einblattdruck Christliche Erinnerung [Augsburg:

Johannes Schultes 1629], in Harms, Flugblätter, Bd. 1, Nr. 236

Tab. 1. Entwicklung der Medizinerzahl im 16. Jahrhundert in Augsburg

(Dieminger 1999, S. 32)

Tab. 2: Versorgung der Augsburger Bevölkerung mit studierten Ärzten

im 16. und beginnenden 17. Jahrhundert (Dieminger 1999, S. 33)

Tab. 3: Versorgung der Augsburger Bevölkerung mit Handwerkschirurgen

im 16. und beginnenden 17. Jahrhundert (Dieminger 1999, S. 37)

Tab. 4: Die medizinische Versorgung der Augsburger Bevölkerung

mit Heilkundigen im 16. und beginnenden 17. Jahrhundert

(Dieminger 1999, S. 37)

\footnotetext{
$\mathrm{BMB}$ - Baumeisterbücher

$\mathrm{CM}$ - Collegium Medicum

StChr - Chroniken der deutschen Städte

StAA - Stadtarchiv Augsburg

SStBA - Stadt- und Staatsbibliothek Augsburg
}

Abkürzungen 


\section{Einleitung}

Als im Jahr 1535 in der Reichstadt Augsburg wieder die Pestepidemie ausgebrochen war, verfassten die Augsburger Stadtärzte Adolph Occo, Johannes Tieffenbach, Gereon Sayler und Wolfgang Talhauser anlässlich dieses Ereignisses gemeinsam eine Druckschrift ${ }^{1}$. Mit ihrer medizinischen Abhandlung traten sie als Pestexperten vor der städtischen Obrigkeit auf, um auf Missstände auf dem Augsburger Gesundheitsmarkt während der Seuchenzeit hinzuweisen. Darüber hinaus forderten sie die hiesige Ärzteschaft zur strengeren Beachtung der galenisch-hippokratischen Medizinlehre bei der Behandlung der Pestkranken auf. Ihr eigentliches Anliegen war aber die Erklärung der Pest, wie schon im Titel ihrer Schrift Was die Pestilentz an jr selbs sey formuliert wurde. Zu diesem Zweck stellten die Stadtärzte in ihrem Werk das damalige Wissen über die Ätiologie, Prophylaxe und Therapie der Pest zusammen. An den Anfang ihrer Ausführungen stellten sie die folgende Definition der Krankheit: Die Pestilentz ist ain fiebrige/ vergiffte/ auch befleckende kranckhait und feülung des hertzens/bluts und gaister so im hertzen seind/ anfengklich und nachfolgend außgetaylt durch die austreybende krefft des hertzens in den gantzen leib ${ }^{2}$. Den Grund für den epidemischen Ausbruch der Pestinfektion sahen die Stadtärzte in der sogenannten Luftvergiftung. Für diese Art der Luftvergiftung waren verschiedene Vorgänge in der Natur verantwortlich. Im Jahr 1535 war nach Ansicht der Ärzte die primäre Ursache in den unregelmäßig verlaufenen Jahreszeiten zu finden. Außer dieser natürlichen Begründung betonten die gelehrten Mediziner ausdrücklich, dass die grundlegende Ursache dieses Krankheitsgeschehens immer im Verhängnis Gottes zu sehen sei: Doch so ist die fürnemst ursach/ hails und widerwertigkait der unerforschlich gerecht will Gottes/der doch zu volziehung seiner hailigen werck, sich dieser und andrer mittel gebraucht/ und kann fruchtbars unfruchtbar/ unfruchtbars aber fruchtbar machen ${ }^{3}$. Daher sollte man neben dem Ergreifen medizinischer Maßnahmen gleichzeitig Gott um die Abwendung des Unheils ersuchen. Diese kurzen Auszüge aus der Augsburger Pestschrift deuten bereits an, dass die Pest in Augsburg des 16. Jahrhunderts gemäß den zeitgenössischen Vorstellungen von Medizin und Religion wahrgenommen und bewertet wurde. Bevor aber das Seuchenphänomen in der Reichstadt Augsburg um 1500 in allen Details analysiert wird, muss man zunächst die Auffassung von der Pest in der modernen Medizin in den Blick nehmen.

\footnotetext{
${ }^{1}$ OCCo/SAYler/TIefFenbach/Talhauser, Was die Pestilentz an jr selbs sey [Augsburg: Philipp Ulhart 1535].

${ }^{2}$ Ebd. S. A III.

${ }^{3}$ Ebd. S. A IV.
} 
Dadurch werden die Analysemöglichkeiten sowie die Grenzlinien der Erkenntnis in der historischen Pestforschung aufgezeigt.

\section{Das Phänomen der Pest in Gegenwart und Vergangenheit - das Problem der retrospektiven Diagnose}

In der modernen Medizinwissenschaft wird mit dem Begriff Pest eine Infektionskrankheit bezeichnet, die vom Bakterium Yersinia pestis ausgelöst wird ${ }^{4}$. Diese Krankheitseinheit stellt dabei eine klassische Zoonose dar, d.h. sie kommt ursprünglich bei einigen Tierarten vor und kann unter bestimmten Bedingungen auf den Menschen übertragen werden. Ein primäres Reservoir der Krankheit bilden nämlich mit dem Bakterium infizierte Nagetiere, darunter hauptsächlich Ratten, die dagegen immun bleiben und nicht erkranken. Kommt es jedoch in ihren Naturherden zu Wanderbewegungen, so können damit auch andere Populationen befallen werden, die eine unzureichende Immunität aufweisen und folglich daran sterben. Die Übertragung des Bakteriums findet dabei durch etwa 30 Floharten statt, worunter der Rattenfloh (Xenopsylla cheopsis) eine besondere Rolle spielt. Bei diesen Flöhen kommt es infolge der sich vermehrenden Pestkultur zu einer Blockade des Mageneingangs, so dass sie dauernd, aber vergeblich Blut zu saugen versuchen und dabei die Pesterreger besonders geballt weitergeben. Falls die infizierten Rattenpopulationen in der Nähe menschlicher Siedlungen aussterben, können die auf ihnen parasitär lebenden Rattenflöhe wegen des Wirtsmangels auf die Menschen überspringen und dabei das Bakterium übertragen. Auch der Menschenfloh (Pulex irritans) ist - wenn auch aufgrund des bei ihnen nicht vorkommenden Blockadephänomens in begrenztem Umfang - in der Lage, die Seuche in der Bevölkerung zu verbreiten.

Nach einer Inkubationszeit von zwei bis sechs Tagen beginnt die Krankheit plötzlich mit hohem Fieber, Kopfschmerzen und Schüttelfrost. An der Stichstelle des Flohbisses, die sich meist an den oberen oder unteren Extremitäten befindet, entwickelt sich ein kleines Bläschen, in dem sich die Pestbakterien in hoher Zahl vermehren. Diese gelangen dann über die afferenten Lymphbahnen zu den nächst gelegenen Lymphknoten der Leiste, der Achselhöhle oder des Halses. Infolge des vermehrten Ausströmens von Blut schwellen die befallenen Lymphknoten schmerzhaft an, wodurch die bläulich verfärbten Beulen entstehen. Damit

\footnotetext{
${ }^{4}$ Gorbach/Bartlett/Blacklow, Infectious Diseases, S. 1568-1574; Groß, Kurzlehrbuch Medizinische Mikrobiologie und Infektiologie, S. 363-365; HAHN/KAUFMANN/Schulz/SuERBAum, Medizinische Mikrobiologie und Infektiologie, S. 264-268; MEYER, Tropenmedizin: Infektionskrankheiten, S. 166-169.
} 
manifestiert sich die erste klinische Form der Krankheit, die Beulenpest, (ca. 75\% aller Erkrankungen), die durch die Übertragungsmodalität des Erregers von Flöhen bestimmt wird. Wenn aber die Filterkapazität der Lymphknoten erschöpft ist, kann diese Abwehrbarriere zusammenbrechen: Die Erreger treten dann in die Blutbahn über und führen folglich zu einer schweren Septikämie. In den infizierten Organen, insbesondere auch in der Haut, kommt es zu starken Blutungen, die zentralnervöse Störungen und Kreislaufversagen verursachen können. Gelangen Erreger über die Blutbahn auch in die Lunge, dann entsteht bei den Kranken die sekundäre Lungenpest, die als die zweite klinische Form der Krankheit definiert ist. Die daraus resultierende Pneumonie bildet eine überaus gefährliche Infektionsquelle, weil die Erreger ausgehustet und nun auch durch Tröpfcheninfektion direkt auf andere Menschen übertragen werden. Die Pestbakterien gelangen auf diese Weise direkt in die Lunge der Kontaktpersonen, bei denen sich entsprechend die primäre Lungenpest entwickelt. Nach einer kurzen Inkubationszeit von einigen Stunden bis zwei Tagen verläuft eine derartige Erkrankung sehr rasch und führt ohne medizinischen Eingriff schon in wenigen Tagen zum Tode. Die Letalität beträgt bei unbehandelten Patienten mit Lungenpest beinahe $100 \%$; bei unbehandelter Beulenpest liegt sie dagegen bei 50-60\%. Deswegen müssen die infizierten Personen nach der Diagnose der Krankheit strikt isoliert und unverzüglich einer medizinischen Behandlung unterzogen werden. Zu diesem Zweck werden therapeutisch bestimmte Antibiotika (u.a. Tetracyclin, Streptomycin, Fluorochinolone und Sulfonamide) eingesetzt, mit denen die Patienten bei der rechtzeitigen Anwendung erfolgreich behandelt werden können. Für Risikopersonen wie medizinisches Personal steht dabei eine Schutzimpfung zur Verfügung.

Diese modernen Therapie- und Prophylaxemethoden beruhen auf den Erkenntnissen der medizinischen Bakteriologie, die sich schrittweise seit der zweiten Hälfte des 19. Jahrhunderts entwickeln konnte. Mit der Anwendung mikrobiologischer Labortechniken wurde die frühere Wahrnehmungsweise der Infektionskrankheiten grundsätzlich verändert, so dass ihre Identität nicht länger über Symptome, sondern ausschließlich über den Erreger bestimmt wurde. Dadurch konnten die einzelnen Krankheitseinheiten genau definiert und dann gezielt mit bestimmten antibiotischen Heilverfahren behandelt werden.

Die wichtigsten Erkenntnisse zur Pest wurden dabei während der großen Pandemie in den asiatischen Ländern gewonnen, die von den 1890er bis in die 1920er nach Schätzungen zehn bis zwölf Millionen Tote forderte ${ }^{5}$. Kurz nach Ausbruch der Seuche wurde das Pestbakterium

\footnotetext{
${ }^{5}$ Dazu ausführlich KUPFERSCHMIDT, Die Epidemiologie der Pest; auch in: CARMICHAEL, Bubonic Plague, S. 628-631; LEVEN, Von Ratten und Menschen, S. 12-16.
} 
1894 von dem Schweizer Tropenarzt Alexandre Yersin (1863-1943) in Hongkong isoliert und nach den Prinzipien der Bakteriologie beschrieben. Der französische Forscher Paul Louis Simond (1858-1947) entdeckte dagegen im Jahr 1898 den Rattenfloh als Hauptüberträger der Krankheit. Nach diesen ersten spektakulären Erkenntnissen benötigte die bakteriologische Forschung noch nahezu 50 Jahre, um die wichtigsten Übertragungswege der Krankheit vollständig aufzuklären und wirksame chemotherapeutische Mittel zu erfinden. Dank diesen langjährigen Anstrengungen der medizinischen Forscher ist die Pest als epidemiologisches Problem heutzutage weitgehend beseitigt, auch wenn das nicht bedeutet, ihre Ursprungsherde unter weiterhin infizierten Nagetieren völlig ausgerottet zu haben.

Die moderne Medizinwissenschaft definiert also die Pest als eine exakt bestimmte Krankheitseinheit, deren Ätiologie, Verlauf sowie mögliche Behandlungsmethoden mikrobiologisch aufgeklärt sind. Diese Tatsache kann leicht zu dem Fehlschluss führen, dass sich dieses biologische Phänomen auch in der Vergangenheit retrospektiv verfolgen lässt ${ }^{6}$. Man muss hier zunächst zwei verschiedene Vorgehensweisen unterscheiden, mit denen die Infektionskrankheiten in der Geschichte erforscht werden können. Eine retrospektive Diagnose im Sinne der modernen Krankheitseinheit ist nämlich nur im Rahmen der Paläopathologie möglich, indem man in organischem Material mit molekulargenetischen Verfahren bakterielle DNA nachweist ${ }^{7}$. Bei einer solchen Untersuchung wurde beispielsweise das Bakterium Yersinia pestis in den Knochenresten von zwei Erwachsenen und einem Kind auf einem Pestfriedhof von 1348 in Montpellier nachgewiesen ${ }^{8}$. Damit konnten aber nur einzelne Krankheitsfälle identifiziert werden, wodurch über den Verlauf der damaligen Epidemie noch keine genauere Aussage getroffen ist. Derartige paläopathologische Befunde gehören dabei in ihrer Aussagekraft zur modernen Medizin, weil sie auf denselben naturwissenschaftlichen Methoden basieren. Aus diesem Grund sind sie für Historiker wenig hilfreich und können das Bild der Seuchengeschichte einzig ergänzen.

Die Geschichtswissenschaft richtet dagegen ihr Augenmerk auf die Analyse der schriftlichen Quellen, die Auskunft über Seuchen in der Vergangenheit geben können. Für die vormikrobiologische Ära sind darin ausschließlich Informationen über die damaligen

\footnotetext{
${ }^{6}$ Zum methodischen Problem der retrospektiven Diagnose in der Medizingeschichte einschlägig: CUNNINGHAM, Transforming plague; LEVEN, Krankheiten - historische Deutung vs. retrospektive Diagnose; DERS., Von Ratten und Menschen, S. 25-32; KINZELBACH, Gesundbleiben, Krankwerden, S. 134-190; STEIN, Franzosenkrankheit, S. 13-24; JANKRIFT, Up dat god sich aver uns verbarmen wolde, S. 61-75.

7 Allgemein zum Thema der Paläopathologie: ROBERTS/MANCHESTER, The Archaeology of Disease; Rothschild/Martin, Paleopathology; LeVEn, Retrospective Diagnosis and Ancient Medical History; Zur paläopathologischen Untersuchung der Pest, vgl. in: DRANCOURT/RAOULT, Molecular insights; MCCORMICK, Rats, Communications, and Plague.

${ }^{8}$ DRANCOURT/RAOULT, Molecular insights, S. 108.
} 
Auffassungen von Krankheit, ihre Symptome, Ausbreitung und Mortalität überliefert. Aber darin sind selbstverständlich keine zuverlässigen Hinweise auf einen bestimmten Erreger im Sinne der modernen Bakteriologie zu erwarten. Der Grund hierfür ist, dass solche historischen Schilderungen keine medizinische Datensammlung darstellen, anhand derer man eine retrospektive Diagnose moderner Krankheitseinheiten aufstellen kann. Vor der Entwicklung der Mikrobiologie in der zweiten Hälfte des 19. Jahrhunderts bestanden andere Erklärungsmodelle für Seuchen, die entsprechend den damaligen kulturspezifischen Wahrnehmungsmöglichkeiten formuliert wurden. Die Vorstellungen von Krankheiten veränderten sich dabei mehrmals in der Geschichte und können daher nicht als konstante Größe angesehen werden. Historisch konstant ist lediglich ihre dauerhafte Veränderung. Immer bestimmten die Gesellschaft und ihre Kultur im weitesten Sinne, wie in verschiedenen Epochen das Phänomen von Krankheit und Gesundheit gedeutet wurde. Dasselbe gilt auch für die gegenwärtige Bakteriologie, die zum allerersten Mal in der Geschichte offensichtliche und nachhaltige Erfolge im Kampf gegen Seuchen erzielen konnte. Die modernen Krankheitseinheiten, die aufgrund der im Labor gewonnenen naturwissenschaftlichen Fakten definiert wurden, bilden auch eine Kulturleistung, sind aber ebenso dem historischen Wandel unterworfen ${ }^{9}$. Unter der geschichtswissenschaftlichen Perspektive kann man die Erkenntnisse der modernen Medizin als „eine von vielen möglichen Formen der Wahrnehmung und Erzeugung von Wirklichkeit" bezeichnen ${ }^{10}$. Die historischen Berichte über das Seuchengeschehen, die in den vormikrobiologischen Medizinsystemen entstanden sind, können daher keinesfalls mit den ärztlichen Befunden der heutigen Medizinwissenschaft gleichgesetzt werden.

Darüber hinaus bestehen bedeutende, im engeren Sinne naturwissenschaftliche Gründe, warum die retrospektive Diagnose der Seuchen in der Geschichte ein fragwürdiges Unterfangen bleibt. Es ist plausibel zu erklären, dass die existierenden Infektionskrankheiten, mit denen die Zeitgenossen während der vergangenen Jahrhunderte konfrontiert wurden, nicht dauerhaft identisch sind ${ }^{11}$. Infolge der biologischen Evolution von Erregern konnte möglicherweise auch die Pathomorphose der durch sie verursachten Infektionskrankheiten verändert werden, wodurch sich ihr früheres Erscheinungsbild und ihre damaligen

\footnotetext{
${ }^{9}$ Die neueren Studien aus der Wissenschaftsgeschichte haben anschaulich gezeigt, welche Faktoren die langwierige Entwicklung der Bakteriologie beeinflussten. Dazu vgl. in: SCHLICH, Wissenschaft. Die Herstellung der wissenschaftlichen Fakten; Wright/TrEACHER, The Problem of Medical Knowledge; LEVEN, Von Ratten und Menschen, S. 31-32.

${ }^{10}$ SCHLICH, Wissenschaft. Die Herstellung der wissenschaftlichen Fakten, S. 123.

11 LEVEN, Krankheiten - historische Deutung vs. retrospektive Diagnose, S. 171; DERS., Von Ratten und Menschen, S. 26; HeRLIHY, Der Schwarze Tod, S. 28.
} 
Übertragungswege durchaus wandelten. Erstmalig in einer Population auftretende Infektionskrankheiten, die in der Forschung als „virgin population epidemics“ bezeichnet werden, verlaufen zunächst verheerender als in der Folgezeit ${ }^{12}$. Dadurch können sich Seuchen in verschiedenen Epochen sehr unterschiedlich auf die Menschenpopulationen auswirken.

Zur Frage der retrospektiven Diagnose der Pest in der Geschichte kann man generell feststellen: Alle Seuchen in der Vormoderne, die in zeitgenössischen Berichten mit dem Namen „Pest““ oder mit anderen dafür synonym angewandten Begriffen bezeichnet wurden, können als Krankheiten betrachtet werden, die nur mit einiger Wahrscheinlichkeit mit der modernen Krankheitseinheit Pest identisch sind. Ebenso gut kann es sich dabei um verschiedene Krankheiten mit ähnlichen Symptomen handeln, die von den Zeitgenossen aufgrund der damaligen Wahrnehmungsmöglichkeiten nicht genauer unterschieden werden konnten. Alle Versuche der Identifikation historischer Krankheitsbilder mit den modernen Mitteln der medizinischen Diagnostik sind deshalb nichts anderes als Spekulationen. Diese Vorhaben sind aus den genannten Gründen bereits von Beginn an zum Scheitern verurteilt, weil sie den historischen Auffassungen, der zeitgenössichen Wahrnehmung und den soziokulturellen Gründen von Krankheiten nicht genügend Aufmerksamkeit widmen und diese nicht in ihrem historischen Entstehungskontext betrachten.

Wie Annemarie Kinzelbach in ihrer Analyse der Bezeichnungen und der Symptombeschreibungen für Epidemien in Ulm und Überlingen im 16. und 17. Jahrhundert und Kay-Peter Jankrift in seiner Fallstudie für die westfälischen und rheinischen Städte im Mittelalter aufzeigen, lassen sich die damaligen Berichte über die Seuchen keinesfalls - im modernen Sinne - genau bestimmten Krankheitseinheiten zuordnen ${ }^{13}$. Die damaligen Beschreibungen von Seuchensymptomen erweisen sich als vieldeutig und liefern keine genauen Hinweise auf eine bestimmte Krankheitsursache. Dieselbe Feststellung lässt sich im Übrigen auch für die moderne Medizinwissenschaft treffen. Keine Infektionskrankheit wird heutzutage ausschließlich nach ihren Symptombeschreibungen identifiziert. Die Identität einer bestimmten Krankheitseinheit kann erst im Labor mit molekulargenetischen Verfahren verifiziert werden, indem ein bestimmter Erreger im organischen Material eines Kranken nachgewiesen wird. Aus diesem Grund ist umso nachdrücklicher dafür zu plädieren, dass überlieferte Seuchenbilder aus dem vormikrobiologischen Zeitalter nicht aus dem Blickwinkel der modernen Medizin gedeutet werden. Die retrospektive Diagnose der Pest kann nur im

\footnotetext{
${ }^{12}$ Ein Beispiel dafür sind Masern und Pocken, die sich bei ihrer Einschleppung nach Amerika in der Neuzeit unter den Indianern und bei Inselpopulationen im verheerenden Ausmaß ausgewirkt haben, dazu in: LE ROY LADURIE, Un concept, S. 682-689.

${ }^{13}$ KINZELBACH, Gesundbleiben, Krankwerden, S. 134-190; JANKRIFT, Up dat god sich aver uns verbarmen wolde, S. 61-75.
} 
Rahmen der Paläopathologie durchgeführt werden, die sich naturwissenschaftlicher Methoden bedient.

Ziel der historischen Pestforschung ist es daher nicht, die von der Mikrobiologie definierte Krankheitseinheit in der Vergangenheit aufzufinden und deren Epidemien bis auf ihren möglichen Ursprung zurückzuverfolgen. Genauso wenig Sinn ergäbe der Versuch, die modernen Erkenntnisse der Medizin auf das damalige Seuchengeschehen zu projizieren, um den Verlauf der früheren Epidemien nach dem heute gültigen medizinischen Maßstab erklären zu können. Die Aufgabe des Historikers besteht vor allem darin, das Phänomen der Pest jeweils in ihrem zeitgenössischen Kontext zu untersuchen. Der Ausgangspunkt der historischen Analyse sind vor allem die Wahrnehmungs- und Deutungsmuster, mit denen die Krankheiten von den Zeitgenossen definiert wurden. Zur Medizingeschichte gehört auch die Untersuchung der sich daraus ergebenden Formen der Reaktionsweisen und Seuchenbekämpfung sowie der verschiedenartigen Auswirkungen der Seuchen auf die Gesellschaft. Mit der Fokussierung des erkenntnisleitenden Interesses auf die Pest in ihrer zeitgenössischen Ausprägung kommt man zu historisch verwertbaren Ergebnissen.

\section{Die soziale Konstruktion der Pest - methodische Grundlagen, Fragestellung und Quellen der Arbeit}

Der methodische Ausgangspunkt der vorliegenden Studie ist das von Martin Dinges herausgearbeitete Modell zur Erforschung der „Sozialen Konstruktion von Seuchen“14. Diesem Forschungsansatz liegt die Vorstellung zugrunde, dass sowohl die Krankheit selbst als auch die Methoden zu ihrer Bewältigung in einer Gesellschaft jeweils konstruiert werden. Dies geschieht in einem komplexen Prozess der Interaktionen zwischen sozialen Akteuren, die sich allgemein in vier Gruppen einordnen lassen:

1) Kranke in einem sozialen Umfeld,

2) Heiler mehr oder minder professioneller Art,

3) Obrigkeiten oder Staaten, die für autoritative Regelungen und deren Durchsetzung in einer Gesellschaft zuständig sind,

4) „an Seuchen Interessierte“, wie die Kirche oder andere Interessengruppen, die in erster Linie nicht durch die Heilfunktion definiert sind.

\footnotetext{
${ }^{14}$ DINGES, Neue Wege, S. 8-20.
} 
Diese Akteure handeln dabei in einer Wechselbeziehung mit der „Umwelt“, in der vor allem der technisch-zivilisatorische Stand einer Gesellschaft (Naturbeherrschung) und die naturräumlichen Gegebenheiten der Pathozönose (Krankheitsspektrum) einbezogen sind ${ }^{15}$. Unter solchen Bedingungen werden die „öffentlichen Meinungen“, d.h. Diskurse und Praktiken von sozialen Akteuren entwickelt, die in einem historisch variablen Machtgefälle zum Einsatz kommen. Dadurch wird im Prozess sozialen Aushandelns der Umgang mit den Krankheiten in einer Gesellschaft endgültig bestimmt.

Das dargestellte Modell von Dinges stellt die wichtigsten Wirkkräfte und Faktoren zusammen, die auf die soziale Konstruktion von Seuchen in allen Epochen einwirken können. Es liefert damit eine allgemeine Grundlage zur Erforschung der Seuchengeschichte, wobei man es jedoch gemäß der speziellen Ausrichtung jeder konkreten Untersuchung modifizieren muss. Aus diesem Grund werden im Folgenden einige Fragen der methodischen Vorgehensweise dargelegt, die für die praktische Umsetzung dieses Forschungskonzeptes in der vorliegenden Studie erklärungsbedürftig sind.

Zum Ersten muss man die Akteure der sozialen Konstruktion der Pest in Augsburg um 1500 genauer charakterisieren, die im handlungstheoretischen Modell in vier allgemeine Kategorien eingeteilt sind. Zur Gruppe der Kranken in einem sozialen Umfeld gehören hier die Pestkranken, ihre Familien, Freunde und Nachbarschaft sowie alle Personen, die mit den infizierten Personen direkt in Kontakt kommen. Die sozialen Differenzen zwischen Armen und Reichen, die im Erkrankungsfall entweder auf die Hilfe anderer Personen angewiesen waren oder sich selbst um ihre Behandlung sorgen konnten, sind von großer Bedeutung. Als „Heiler“ sind die gelehrten Ärzte, die Handwerkschirurgen wie Barbiere, Wundärzte und Bader, die unprofessionellen Laienheiler, die Apotheker sowie das andere Medizinalpersonal zu nennen, das bei der Pflege der Pestkranken in der Stadt bzw. in den Pesthäusern tätig war. Die Gruppe der „Obrigkeiten“ umfasst den Rat, die Ratsämter sowie alle städtischen Verordneten, die an der Anordnung oder Ausführung der obrigkeitlichen Pestpolitik in Augsburg direkt beteiligt waren. Die letzte Gruppe, in der grundsätzlich alle anderen an der Pest „Interessierten“ zusammengestellt werden, stellt sich am vielfältigsten dar. Darunter sind

\footnotetext{
${ }^{15}$ Unter dem Begriff „Pathozönose“ (wörtlich „Miteinander/Gemeinschaft von Krankheiten“) ist vornehmlich das Auftreten einer Reihe von Krankheiten verstanden, die zu bestimmten Zeiten in einer Gesellschaft lebensbedrohend waren und sich dann in ihrer Virulenz veränderten, verschwanden oder durch neue Krankheiten ersetzt wurden. Diesen neuen Begriff prägte Mirko Grmek, der das Krankheitsspektrum der griechischen Antike auf der Basis von Textquellen und paläopathologischen Befunden nach modernen Krankheitseinheiten zu rekonstruieren versuchte, in: GRMEK, Diseases; Kritisch zu diesem Versuch in: SHAW, Grmek's Pathological; LEVEN, Retrospective Diagnosis and Ancient Medical History. Weil diesem Forschungskonzept der Gedanke der retrospektiven Diagnose von Krankheiten in der Vergangenheit zugrunde liegt, wird es in der vorliegenden Studie abgelehnt.
} 
zunächst die Geistlichen zu erwähnen, die vornehmlich für die religiöse Bewältigung der Pest zuständig waren. Dabei sind die diesbezüglichen Veränderungen während der Reformationszeit in Augsburg zu beachten. Außerdem lässt sich im Fall der Pestepidemien voraussetzen, dass alle Stadtbewohner auf irgendeine Weise davon betroffen sein konnten. Die allgemeine Interessengrundlage bildete in diesem Zusammenhang eine potentielle Ansteckungsgefahr, die aus der enormen Virulenz der damaligen Krankheit resultierte.

Bei näherem Betrachten zeigt sich auch, dass die sozialen Akteure im Forschungsmodell keinesfalls als homogene Gruppen anzusehen sind, sondern dass sich diese vielmehr vielschichtig gestalten. Sie können sich auch unter bestimmten Voraussetzungen überschneiden, sodass die ihnen zugeordneten Personen gleichzeitig mehr als nur einer Kategorie angehören ${ }^{16}$. Schon aufgrund der möglichen Erkrankung oder betroffener individueller Interessen in der Pestzeit sind die Grenzen zwischen diesen vier Gruppen häufig als fließend zu betrachten.

Darüber hinaus sollen die sozialen Akteure auch bezüglich ihrer Relevanz im Prozess der sozialen Konstruktion der Pest differenziert werden. Unter einer solchen Perspektive ist die Gruppe der Obrigkeiten anstelle der Gruppe der Kranken in einem sozialen Umfeld an die Spitze des oben genannten Vierecks zu stellen ${ }^{17}$. Seit der Entwicklung der obrigkeitlichen Pestpolitik, die den Umgang mit der Seuche in der gesamten Stadtgemeinde weitgehend prägte und gestaltete, ist die städtische Obrigkeit zwar ein nicht alles entscheidender, dafür aber ein besonders wichtiger Akteur.

Zum Zweiten wird hier das soziale Aushandeln öffentlicher Meinungen näher erörtert, das zwischen allen an diesem Prozess beteiligten Akteuren stattfindet. Eine grundlegende Voraussetzung für die folgenden Ausführungen ist die Feststellung, dass ein Krankheitsphänomen jeweils aufgrund der kulturspezifischen Wahrnehmungen expliziert wird, wodurch sich in einem gegebenen historischen Kontext eine beschränkte Anzahl von bestimmten Denkformen entwickeln kann. Demnach sind zunächst die Konzepte und Deutungen der Pest herauszuarbeiten, die in der vormikrobiologischen Ära formuliert wurden. Die damalige Vorstellungswelt lässt sich dabei allgemein in zwei Diskurse, den religiösen und den medizinischen, einordnen, die in einer engen Wechselbeziehung zueinander standen und

\footnotetext{
${ }^{16}$ Auf diese zwei Aspekte der schematischen Aufteilung von sozialen Akteuren wies schon Kay-Peter Jankrift in seiner Habilitationsschrift hin, der sich in seiner Untersuchung auf das Forschungsmodell von Dinges stützte, JANKRIFT, Up dat god sich aver uns verbarmen wolde, S. 30.

${ }^{17}$ Im Modell von Dinges wurden die Kranken in einem sozialen Umfeld als erster Akteur genannt, um vor allem die Vernachlässigung der Patientenrolle in der bisherigen Forschung deutlich hervorzuheben, DINGES, Neue Wege, S. 9-11. Einschlägig zur Erforschung der Kranken im sozialen Umfeld in der Frühen Neuzeit am Beispiel der Stadt Köln: JÜTTE, Ärzte, Heiler und Patienten.
} 
im Laufe der Zeit einigen Veränderungen unterlagen. Im Zentrum dieser Analyse steht insbesondere die Frage, wie sich diese zwei Diskurse in der Reichsstadt Augsburg verbreiteten und damit die Seuchenbewältigung in der Stadtgesellschaft bestimmten. In diesem Zusammenhang ist jedoch hervorzuheben, dass die auf dem zeitgenössischen Wissensbestand über die Pest beruhenden Praktiken gleichzeitig auch von verschiedenartigen Handlungsmotivationen der Zeitgenossen beeinflusst waren. Die einzelnen Akteure richteten sich nämlich während der Epidemien auch nach ihren individuellen und häufig widersprüchlichen Interessen, die im Prozess der sozialen Interaktionen geregelt wurden. Welche Praktiken sich im Umgang mit der Pest endgültig durchsetzen konnten, war vor allem von der damaligen Macht- und Sozialordnung abhängig. Die vielfältigen Interessen von sozialen Akteuren sind also im Kontext der damaligen Diskurse und Praktiken immer zu berücksichtigen, wenn ihre komplexen Implikationen analysiert werden sollen.

Das oben dargestellte handlungstheoretische Modell lässt sich exzellent für die Erforschung der Pest in einer vormodernen Stadtgesellschaft anwenden. Im Fall dieser Krankheit war nämlich die größtmögliche Anzahl von sozialen Akteuren davon betroffen, wodurch dieses Phänomen zugleich auf vielen Ebenen untersucht werden kann. Die Auswahl nur einer Stadt für die Untersuchung gewährleistet dabei, dass alle Akteure einem kohärenten Raum angehören und sich miteinander in einem ständigen kommunikativen Austausch befinden. Dadurch können die Interaktionen zwischen allen Beteiligten in einer Fallstudie näher analysiert werden. Diesen komplexen Prozess der sozialen Konstruktion der Pest am Beispiel der Reichstadt Augsburg zu erforschen, ist das Grundziel der vorliegenden Dissertation. Der Zeitrahmen der Untersuchung umfasst die Zeitperiode seit den ersten in Augsburg belegten Pestepidemien in der zweiten Hälfte des 14. Jahrhunderts bis zur Mitte des 16. Jahrhunderts, als sich die städtischen Maßnahmen zur Bewältigung der Pest in vollem Ausmaß etablierten. Der Schwerpunkt der Analyse liegt dabei auf dem kulturellen Wandel im Umgang mit der Pest, der sich in Augsburg um 1500 vollziehen konnte. Die Hauptthese der Arbeit ist es, dass sich mit der Entwicklung der obrigkeitlichen Pestpolitik am Ende des 15. Jahrhunderts die soziale Konstruktion der Pest in Augsburg herausbilden konnte, deren Grundlagen von einem engen Zusammenspiel des religiösen und medizinischen Diskurses geprägt waren. Mit der damals schrittweise vorgehenden Rezeption von medizinischen Vorstellungen kam es zu einem fundamentalen Wandel im Umgang mit der Pest. Neben der nach wie vor allgemeingültigen Wahrnehmung der Seuchen durch die Religion wurde dieses Krankheitsphänomen seither auch nach den Deutungsmustern der zeitgenössischen Medizin 
grundlegend bewältigt. Die vorliegende Studie wird damit einen Beitrag zur epochenspezifischen Wahrnehmung der Pest, zur städtischen Seuchenbekämpfung sowie zur Hospitalgeschichte in der Vormoderne leisten.

Die Untersuchung beginnt mit der Analyse von Augsburger Quellenbegriffen, die von den Zeitgenossen für die Bezeichnung der Pest verwendet wurden. Ausgehend von der chronologischen Darstellung der ersten Epidemien werden dann die Grundzüge der Wahrnehmung und Bewältigung der Pest sowie das städtische Medizinalwesen im spätmittelalterlichen Augsburg charakterisiert (Kapitel II). Nach diesem Auftakt ist ein umfangreicher Teil der Untersuchung den zeitgenössischen Diskursen über die Pest gewidmet, die seit der Mitte des 14. Jahrhunderts von den Geistlichen und Medizinern formuliert wurden. Die damalige Vorstellungswelt über die Pest ist in der ersten Linie anhand der gedruckten medizinischen und religiösen Pestschriften herauszuarbeiten, die bis zur Mitte des 16. Jahrhunderts in Augsburg gedruckt oder von Augsburger Autoren verfasst wurden (Kapitel III). Die Grundsätze der medizinischen Pestlehre, denen das antike Medizinkonzept der Viersäftelehre zugrunde lag, werden hinsichtlich der Ätiologie der Pest durch die Vergiftung der Luft, der astrologischen und natürlichen Ursachen dafür sowie der Vorstellungen über die Ansteckung mit der Pest dargelegt (Kapitel IV). In diesem Zusammenhang wird auch das Verhältnis zwischen Medizin und Religion näher behandelt. Die Vorstellung von der Pest als Gottes Strafe und die damit verbundene Verehrung von Pestheiligen sind auch im Hinblick auf die diesbezüglichen Veränderungen während der Reformation darzustellen. Das Thema des nächsten Kapitels bilden die medizinischen Anweisungen in den Pestschriften (Kapitel V). Darunter werden die Schutzmaßnahmen vor der verdorbenen Luft und vor der von den Pestkranken ausgehenden Ansteckungsgefahr besprochen. Die Prophylaxe mittels der richtigen Diätetik und der Selbstmedikation sowie die von den Medizinern angewandten Methoden der Behandlung für die Pestkranken sind auch an dieser Stelle zu erörtern. Darüber hinaus werden die Belehrungen von protestantischen Theologen zur Flucht und zum Selbstschutz während der Pestzeit thematisiert, die die damaligen medizinischen Anweisungen maßgebend beeinflussten. Nach der Untersuchung der zeitgenössischen Denkformen stehen die Anfänge der städtischen Pestpolitik in Augsburg nach den Grundsätzen der damaligen Medizinlehre im Zentrum der Analyse (Kapitel VI). Mit der Anlegung des Unteren Gottesackers und der Einrichtung der Pesthäuser im Jahr 1494 werden die ersten städtischen Maßnahmen zur Pestbewältigung dargestellt, die damals nach der Vereinbarung mit den kirchlichen Machtträgern unternommen wurden. Während der Epidemie von 1521 sind dagegen die städtischen Anordnungen zu verfolgen, die vom Rat 
direkt nach den Anweisungen von Augsburger Stadtärzten erlassen wurden. Die Gründung des großen Pesthospitals (die sogenannten „Brechhäuser") und die erste städtische Pestordnung werden ausführlich in diesem Kapitel dargelegt.

Danach ist der weitere Ausbau der obrigkeitlichen Pestbewältigung in Augsburg bis zur Mitte des 16. Jahrhunderts zu analysieren (Kapitel VII). Dazu gehören vor allem die Anlegung des Oberen Gottesackers unter dem Widerstand der Augsburger Kirchherren während der Reformationszeit, die Aufstockung des städtischen Dienstpersonals in der Pestzeit sowie die erste gedruckte Pestordnung. Darüber hinaus werden auch die verschärften Maßnahmen zur Pestbewältigung während des Augsburger Reichstages von 1547-1548 und die Anordnungen zum Bettelwesen im Kontext der Seuchenbewältigung in der ersten Hälfte des 16. Jahrhunderts dargelegt. Zum Abschluss der Studie werden alle historischen Faktoren in den Blick genommen, die sich auf die soziale Konstruktion der Pest um 1500 auswirken konnten (Kapitel VIII). Dabei werden insbesondere der Aufstieg von gelehrten Medizinern zu städtischen Experten und ihre Rolle bei der Regelung des städtischen Medizinalwesens analysiert. Die Entstehung der städtischen Pestpolitik im Kontext der Kommunalisierung der Armen- und Krankenfürsorge bildet den letzten Punkt der Untersuchung.

Eine Studie zur sozialen Konstruktion der Pest erfordert eine große Vielfalt in der historischen Überlieferung, die gleichzeitig Einblicke in die damalige Vorstellungswelt wie auch in die alltäglichen Praktiken von Zeitgenossen vermitteln kann. Dabei soll es möglich sein, die komplexen Interaktionen zwischen sozialen Akteuren in der Pestzeit detailliert herauszuarbeiten. In dieser Hinsicht stellt sich die Quellenlage zur Pestgeschichte in Augsburg besonders günstig dar, wenn sie auch nicht für den gesamten Untersuchungszeitraum eine gleichbleibende Dichte aufweist. Die Informationen über die ältesten Epidemien stammen vornehmlich nur aus den chronikalischen Schilderungen, die meistens in kurzen Einträgen über das damalige Pestgeschehen berichten. Die Chroniken bilden dabei die erste Quellengruppe, die in der vorliegenden Studie ausgewertet wurde. Viele Texte wurden im Rahmen der Editionsreihe für die Chroniken der deutschen Städte vom 14. bis zum 16. Jahrhundert herausgegeben ${ }^{18}$. Die zweite Quellengattung sind die medizinischen und religiösen Pestschriften, die in Augsburg gedruckt oder von Augsburger Autoren verfasst wurden. Solche Druckschriften sind schon seit der zweiten Hälfte des 15. Jahrhunderts zahlreich überliefert und können zur Rekonstruktion der zeitgenössischen Vorstellungen über die Pest herangezogen werden. Die Untersuchung basiert ebenso auf in der Forschung bisher nicht ausgewerteten Archivalien, die im Stadtarchiv und teilweise auch in der Stadt- und

\footnotetext{
${ }^{18}$ StChr, Bd. 4, 5, 22, 23, 25, 29, 32, 33, 34.
} 
Staatsbibliothek in Augsburg aufbewahrt sind. Dieses reichhaltige Aktenmaterial setzt nämlich schrittweise mit der Entwicklung der obrigkeitlichen Pestpolitik um 1500 ein und ist bis zur Mitte des 16. Jahrhunderts in immer größerer Menge vorhanden. Aus den verschiedenen Aktensammlungen wurden vor allem die Ratsprotokolle, die städtischen Pestordnungen, die Verordnungen des Rates sowie die Schreiben der Stadtärzte an den Rat untersucht, in denen Informationen über die Praktiken während der Epidemien festgehalten sind. Darüber hinaus werden in der Studie auch vielfältige Bildquellen berücksichtigt, die sich auf das Phänomen der Pest in der Reichsstadt Augsburg beziehen. Darunter sind religiöse Einblattdrucke, Bilder der Pestheiligen, medizinische Ansichtstafeln zur Behandlung der Pestkranken, Augsburger Stadtpläne sowie Darstellungen der städtischen Pesthäuser zu nennen.

\section{Forschungsstand zur Pestgeschichte}

Die historische Pestforschung gilt schon seit langem als ein klassisches Thema der Medizingeschichte. In einer nahezu unüberschaubar gewordenen Zahl von Publikationen wurde das Phänomen der Pest aus den verschiedensten Blickwinkeln betrachtet. Um die vorliegende Studie in dieser umfangreichen Forschungslandschaft zu verorten, werden nun die thematischen und methodischen Richtungen in der Pestgeschichte präsentiert, ohne dabei einen Anspruch auf die vollständige Zusammenstellung diesbezüglicher Untersuchungen zu erheben. Der Schwerpunkt liegt auf dem deutschsprachigen Forschungsraum mit besonderer Berücksichtigung der Arbeiten zur Pest in der Reichstadt Augsburg.

Das Interesse der Historiker für die Seuchengeschichte wurde schrittweise mit den mikrobiologischen Entdeckungen in der zweiten Hälfte des 19. Jahrhunderts geweckt ${ }^{19}$. Als die biologische Realität der Infektionskrankheiten endgültig aufgedeckt wurde, etablierte sich in der geschichtswissenschaftlichen Forschung die feste Überzeugung, dass eine moderne Krankheitseinheit eine eigene Geschichte hat. Anhand der historischen Schilderungen versuchte man daher die Seuchen bis auf ihren möglichen Ursprung zurückzuverfolgen. In diesen ersten Forschungsansätzen wurde implizit vorausgesetzt, dass Krankheit im Grunde genommen ein unveränderliches und dadurch objektives Phänomen ist, das sich in

\footnotetext{
19 Damals wurden vor allem allgemeine Gesamtdarstellungen zur Seuchengeschichte verfasst: HAESER, Lehrbuch der Geschichte der Medizin; HECKER, Die großen Volkskrankheiten des Mittelalters; LERSCH, Geschichte der Volksseuchen. Daneben auch eine informationsreiche Untersuchung zur Pestgeschichte in Deutschland: HoENIGER, Der Schwarze Tod.
} 
verschiedenen Epochen retrospektiv beobachten lässt. Das Augenmerk der Geschichtswissenschaft richtete sich vor allem auf die Erforschung der Infektionskrankheit, die in der Geschichte als die Seuche schlechthin gilt: die Pest ${ }^{20}$. In diesen Studien wurden Themenfelder behandelt, die die historische Pestforschung für lange Jahrzehnte geprägt haben: die retrospektive Diagnose, die Chronologie der Epidemien und die institutionelle Seuchenbekämpfung. Die Angaben über die Pest in der Vergangenheit, in der man die moderne Krankheitseinheit $\mathrm{zu}$ erkennen glaubte, wurden in chronologischer Reihenfolge meistens nach geographischen Kriterien zusammengestellt ${ }^{21}$. Die damaligen Forschungen wurden auch von der Vorstellung eines erfolgreichen Fortschritts der medizinischen Erkenntnisse getragen, die sich endgültig in der $\mathrm{zu}$ untersuchenden institutionellen Seuchenbekämpfung manifestiert hat. Zur Erklärung des historischen Seuchengeschehens wurden dabei die modernen sozusagen „objektiven“ Erkenntnisse aus den Naturwissenschaften herangezogen. Mit der Übertragung der heutigen medizinischen Denkmuster wurden die bakteriologischen Faktoren zur Verbreitung der Seuchen, ihre klinischen Symptome oder die Wirksamkeit der früheren Heilverfahren während der vormodernen Epidemien analysiert ${ }^{22}$. Erwähnenswert sind auch die Untersuchungen zur vormodernen Pest- und Seuchenlehre. Die Arbeiten von Karl Sudhoff sind nach wie vor das wichtigste bibliographische Hilfsmittel zu den medizinischen Abhandlungen über die Pest im Spätmittelalter ${ }^{23}$.

Die Richtungen der damaligen Seuchenforschung resultierten in erster Linie aus der institutionellen Stellung der Medizingeschichte in Deutschland, die vor allem an medizinischen Fakultäten erforscht wurde. Die Fragestellungen der sich mit der Geschichte beschäftigenden Mediziner konzentrierten sich schwerpunktmäßig auf Themen, die die historische Entwicklung der Heilkunde in allen ihren Aspekten betrafen. Im Mittelpunkt stand dabei die allmähliche Durchsetzung von immer mehr Professionalität, Staatlichkeit und Naturwissenschaft im Medizinalwesen. Andererseits begriff sich um diese Zeit die an den philosophischen Fakultäten angesiedelte allgemeine Geschichtswissenschaft vornehmlich als

\footnotetext{
${ }^{20}$ Aus den ersten Publikationen waren vor allem die folgenden von größter Bedeutung für die künftige Forschung: DiEUdonNÉ, Die Pest in den letzten Jahrhunderten; NoHL, Der schwarze Tod; STICKER, Abhandlungen aus der Seuchengeschichte und Seuchenlehre, Bd. 1.

${ }^{21}$ Darunter ist in der ersten Linie die systematische Studie von Jean-Noel Biraben zu nennen, der einen chronologischen Überblick über die Pestepidemien in Europa erarbeitet hat: BIRABEN, Les hommes et la peste.

${ }^{22}$ WoehlKens, Pest und Ruhr im 16. und 17. Jahrhundert; RoDENwALDT, Die Gesundheitsgesetzgebung des Magistrato della Sanitá Venedigs; DERS., Pest in Venedig 1575-1577.

${ }^{23}$ SudHOFF, Pestschriften aus den ersten 150 Jahren nach der Epidemie des „schwarzen Todes“ 1348; DERS., Deutsche medizinische Inkunabeln.
} 
Geschichte des politischen Geschehens und der Institutionen. In diesem historiographischen Kontext der Medizingeschichte wurden auch die ersten Studien zur Pestgeschichte abgefasst.

Die neuen Themen und Methoden in der historischen Seuchenforschung kamen erst aus der Sozialgeschichte, die sich in Deutschland seit der Mitte der 1970er Jahre etablierte ${ }^{24}$. Mit der sozialgeschichtlichen Wende in der Geschichtswissenschaft wurde das Forschungsinteresse auf die vielfältigen Wechselwirkungen zwischen Gesellschaft und Medizin gelenkt. Damit wurden die Auswirkungen der Pest auf das soziale, wirtschaftliche, politische oder alltägliche Leben untersucht ${ }^{25}$. Dazu wurden zahlreiche Studien im Hinblick auf einzelne Pestepidemien und deren Auswirkungen in verschiedenen Ländern und Städten durchgeführt $^{26}$. Dabei wurde insbesondere der „Schwarze Tod“, d.h. die erste große Pestwelle in der Mitte des 14. Jahrhunderts, analysiert, deren Bedeutung für die europäische Geschichte man ausführlich diskutierte ${ }^{27}$. Die religiösen Aspekte in der Pestgeschichte, wie z.B. die Rolle der Heiligenverehrung oder die frommen Praktiken während der Epidemien, wurden auch thematisiert $^{28}$. Darüber hinaus kommt in der aktuellen historischen Seuchenforschung den Arbeiten ein besonderer Stellenwert zu, die in interdisziplinärer Kooperation entstanden $\operatorname{sind}^{29}$. In diesen Sammel- bzw. Tagungsbänden wurden die sozialgeschichtlichen Methoden für die historische Seuchenforschung in ihrer Gesamtheit erörtert und viele Themen exemplarisch aufgenommen.

Dank der Sozialgeschichte wurde auch die Frage der retrospektiven Diagnose kritisch diskutiert. In dieser Debatte wurden zunächst verschiedene Hypothesen in Betracht gezogen: z.B. ob es sich bei den historisch bekannten Pestepidemien immer um dieselbe moderne Krankheitseinheit handeln konnte oder ob möglicherweise verschiedene infektiöse Krankheiten zur gleichen Zeit ausbrechen konnten $^{30}$. In den 1990er Jahren wurde die Diskussion weiter fortgesetzt und die Identität der Pest in der Geschichte endgültig in Frage

\footnotetext{
${ }^{24}$ Die Überblicksdarstellung zu den verschiedenen Aspekten der Sozialgeschichte der Medizin: LABISCH/SPREE, Neuere Entwicklungen und aktuelle Trends in der Sozialgeschichte der Medizin in Deutschland.

25 Bulst, Der Schwarze Tod. Demographische, wirtschafts- und kulturgeschichtliche Aspekte der Pestkatastrophe von 1347-1352; GRAUS, Pest, Geißler, Judenmorde.

${ }^{26}$ HÖHL, Die Pest in Hildesheim; SchwarZ, Die Pest in Bremen; HatJe, Leben und Sterben im Zeitalter der Pest; IBS, Die Pest in Schleswig-Holstein von 1350 bis 1547/48; BENEDICTOW, Plague in the Late Medieval Nordic Countries.

${ }^{27}$ BERGDOLT, Der Schwarze Tod in Europa; ZINN, Kanonen und Pest; HerLIHY, Der Schwarze Tod; CoHN, The Black Death Transformed.

${ }^{28}$ BULST, Heiligenverehrung in Pestzeiten; DORMEIER, Laienfrömmigkeit in den Pestzeiten des 15./16.

Jahrhunderts; ESSER, Pest, Heilsangst und Frömmigkeit.

${ }^{29}$ Bulst/Delort (Hrsg.), Maladies et société (XIIe-XVIII siècle); RANGER/SLACK (Hrsg.), Epidemics and Ideas; Dinges/Schlich (Hrsg.), Neue Wege in der Seuchengeschichte; MEIER (Hrsg.), Pest. Die Geschichte eines Menschheitstraumas; Ulbricht (Hrsg.), Die leidige Seuche: Pest-Fälle in der Frühen Neuzeit; WAHRMANn/BuchSTEINER/ STRAHL (Hrsg.), Seuche und Mensch.

${ }^{30}$ Slack, The impact of plague, S. 54-78; CARMichaEL, Plague and the poor, S. 27-89; TwIGG, The Black Death in England; SoURNIA, Discipline du diagnostic retrospective.
} 
gestellt $^{31}$. Vor allem aus methodologischen Gründen sowie aufgrund der Quellenanalyse stellte man fest, dass sich die mikrobiologisch definierten Krankheitseinheiten anhand der historischen Berichte keinesfalls eindeutig erkennen lassen. Gleichzeitig wurde aber auch erkannt, dass eine retrospektive Identifikation der Krankheiten nicht nötig ist, um die Bedeutung der Epidemien für die vormodernen Gesellschaften untersuchen zu können.

Im Kontext derartiger methodischer Überlegungen entwickelte sich in der Medizingeschichte das Forschungspostulat, dass man die soziale Konstruktion von Krankheiten in verschiedenen Epochen untersuchen sollte ${ }^{32}$. Dieses Konzept basiert auf der erkenntnistheoretischen Feststellung, dass ein direkter, objektiver Zugang zur Wirklichkeit nicht möglich ist. Der Erkenntnisprozess wird nämlich immer von menschlichen Wahrnehmungsmöglichkeiten gesteuert, die abhängig von verschiedenen sozialen und kulturellen Faktoren unsere Vorstellungen von der Wirklichkeit hervorbringen. Unter dieser Perspektive sind Krankheitsphänomene nicht als eine unveränderbare Konstante der Naturgeschichte zu betrachten. Sowohl die Krankheit selbst als auch die Methoden zu ihrer Bewältigung werden als Teil der sozialen Wirklichkeit von vielen Akteuren und unter bestimmten Bedingungen in einem interaktiven Prozess hergestellt. Mit diesem Forschungsansatz wird den Krankheiten keinesfalls die reale Existenz abgesprochen, sondern es werden die dem kulturellen Wandel unterliegenden Veränderungen in ihrer Wahrnehmung ausdrücklich betont. Zur Erforschung der sozialen Konstruktion von Seuchen wurde das vorher dargestellte Untersuchungsmodell von Martin Dinges herausgearbeitet, das in der vorliegenden Dissertation angewendet wird ${ }^{33}$.

Zur Erforschung der Pestgeschichte der Reichsstadt Augsburg liegt bisher lediglich die ältere Studie von Karl Fries vor, in der er sich mit dem Seuchengeschehen während des Dreißigjährigen Krieges beschäftigte ${ }^{34}$. Daneben untersuchte auch Bernd Roeck in seiner Habilitationsschrift zur Geschichte Augsburgs die letzten Pestepidemien in Augsburg von 1627/28 und 1634/35 insbesondere im Hinblick auf ihre demographischen und sozialen Folgen $^{35}$. Außer diesen Arbeiten wurde die Pest in anderen Monographien zum Augsburger

\footnotetext{
${ }^{31}$ Cunningham, Transforming plague; LEVEn, Krankheiten - historische Deutung vs. retrospektive Diagnose; DERS., Von Ratten und Menschen, S. 25-32; KInZELBACH, Gesundbleiben, Krankwerden, S. 134-190.

${ }^{32}$ Dazu einschlägig: Wright/TrEACHER, The Problem of Medical Knowledge; LACHMUnd/StOLLBERG (Hrsg.), The Social Construction of Illness; LEVEN, Krankheiten - historische Deutung vs. retrospektive Diagnose; DERS., Von Ratten und Menschen, S. 25-32; SCHLICH, Wissenschaft. Die Herstellung der wissenschaftlichen Fakten.

${ }^{33}$ Dinges, Neue Wege, S. 8-20. Dazu vgl. auch die Studien von Dinges über das Verhältnis zwischen der Pest und dem Staat in der sozialen Konstruktion der Pest: DERS., Pest und Staat: Von der Institutionengeschichte zur sozialen Konstruktion; DERS., Pest und Pestpolitik in der europäischen Neuzeit; An diesem Forschungsmodel orientierte sich auch die Studie zur Seuchengeschichte in den westfälischen und rheinländischen Städten: JANKRIFT, Up dat god sich aver uns verbarmen wolde; sowie das Wolfenbütteler Ausstellungskatalog zur Seuchengeschichte in der Frühen Neuzeit: FEUERSTEIN-HERZ (Hrsg.), Gotts verhengnis und seine straffe.

${ }^{34}$ FRIES, Pest zu Augsburg.

${ }^{35}$ ROECK, Krieg und Frieden.
} 
Medizinalwesen nur am Rande erforscht. Eine besondere Stelle nimmt darunter die sozialgeschichtliche Dissertation von Claudia Stein über die Behandlung der Franzosenkrankheit im frühneuzeitlichen Augsburg ein, in der u.a. die Frage der retrospektiven Diagnose diskutiert wurde $^{36}$. Mitchell Hammond stellte dagegen einen Überblick über den Augsburger Gesundheitsmarkt in der Frühen Neuzeit her und Gerhard Gensthaler befasste sich mit dem Medizinalwesen der Reichsstadt bis zum 16. Jahrhundert mit dem Schwerpunkt auf dem Apothekenwesen ${ }^{37}$. In den übrigen Studien zur Medizingeschichte in der Reichstadt Augsburg wurden die gelehrten Ärzte ${ }^{38}$, Bader, Barbiere und Wundärzte ${ }^{39}$, Hebammen ${ }^{40}$, die städtische Armen- und Krankenfürsorge ${ }^{41}$ und das HeiligGeist-Spital $^{42}$ in verschiedenen Aspekten thematisiert. Begleitend zur 2016 im Stadtarchiv München organisierten Ausstellung über den Umgang mit Seuchen in Augsburg, München und Nürnberg im Zeitraum vom Mittelalter bis ins 20. Jahrhundert erschien ein illustrierter Katalog ${ }^{43}$.

\footnotetext{
${ }^{36}$ STEIN, Franzosenkrankheit.

${ }^{37}$ HAMMOND, Origins; GensthaLeR, Medizinalwesen.

${ }^{38}$ MARTZ, Gesundheitswesen; HEINDL-HEINZ IDAMARIE, Gesundheitswesen.

39 Dieminger, Bader; HofFMANN, Augsburger Bäder; ECKER-OFFENHÄUßER, "Pest, Franzosen, Scharbock"; DIES., Joseph Schmid.

${ }^{40}$ BLANKENBURG, Hebammenwesen.

${ }^{41}$ Bisle, Armenpflege; Clasen, Armenfürsorge (1-2); DerS., Arm und Reich; KIEßLING, Gesellschaft, S. 159175, 215-235; LENGLE, Spitäler; SEIDA UND LANDSBERG, Historisch-statistische Beschreibung; WERNER, Stiftungen; TROMETER, Pilgerhaus; PIETSCHMANN, Armen- und Krankenwesen.

${ }^{42}$ LENGLE, »Gottberatbuch«(; DERS., Augsburger Heilig-Geist-Spital; Wüst, Armut und Besitz.

${ }^{43}$ FeldmanN/ Walser/ LANDOIS/ PFOTENHAUER (Hrsg.), Der Feind in der Stadt.
} 


\section{Das Pestgeschehen im spätmittelalterlichen Augsburg}

Mit dem Ausbruch des Schwarzen Todes in den Jahren 1347-1351 beginnt die Geschichte der Pest im mittelalterlichen Europa ${ }^{44}$. Das ungestüme Einreißen dieses neuen Krankheitsphänomens, das sich vor allem durch sehr hohe Virulenz und Mortalität von den anderen im Mittelalter bekannten Krankheiten unterschied, hatte verheerende Bevölkerungsverluste zur Folge, die die Entwicklungsrichtung der mittelalterlichen Wirtschaft, Sozialordnung und Kultur beachtlich mitprägten. Diese bisher unbekannte Seuche wirkte sich mit ihrer enormen Ansteckungs- und Todesgefahr auf die Zeitgenossen aus und musste daher von ihnen als ein neues Lebensphänomen gedeutet und zwingend in die Deutung ihrer Umwelt einbezogen werden ${ }^{45}$. Der Schwarze Tod blieb kein einmaliges Ereignis, sondern wurde zu einer in unregelmäßigen Abständen wiederkehrenden Bedrohung für die menschliche Existenz im Spätmittelalter und in der Frühen Neuzeit. Im Folgenden wird analysiert, aufgrund welcher kulturspezifischen Wahrnehmungen das Pestphänomen in der spätmittelalterlichen Zeitperiode in Augsburg expliziert und bewältigt wurde. Die Überlieferung zum Pestgeschehen im Spätmittelalter beschränkt sich dabei vor allem auf chronikalische Aufzeichnungen, anhand derer man Einblicke in die jeweiligen Vorgänge und Reaktionen im Umfeld des Pestausbruchs gewinnen $\mathrm{kann}^{46}$. In den Augsburger Ratsprotokollen finden sich keine Informationen über die spätmittelalterlichen Epidemien. Dagegen könnten die umfangreichen Bestände an Steuerbüchern und Baumeisterrechnungen vor allem zu statistischen Untersuchungen herangezogen werden, die allerdings in dieser Studie keinen Schwerpunkt bilden. Eine derartige Quellenlage für die spätmittelalterliche Seuchengeschichte ist dabei nicht nur für die Stadt Augsburg charakteristisch, sondern stellt sich auch für andere Städte aus dem deutschsprachigen Raum ähnlich dar ${ }^{47}$.

\footnotetext{
${ }^{44}$ Die erste spätmittelalterliche Pestepidemie in den Jahren 1347-1351, die in der Forschung meistens als „der Schwarze Tod" bezeichnet wird, ist die am häufigsten erforschte Zeitperiode der Pestgeschichte. Zu ihr sind zahlreiche Arbeiten und Monographien erschienen; einschlägige Studien dazu bieten vor allem: BERGDOLT, Der Schwarze Tod; DERS, Pest; Graus, Pest; Herlihy, Der Schwarze Tod; Horrox, The Black Death; NAPHY/SPICER, Der Schwarze Tod; ZINN, Kanonen.

${ }^{45}$ Die Erinnerung an die Justinianische Pest von 541-543 und die danach folgenden Pestwellen während des 6. Jahrhunderts im Oströmischen Reich und in West- und Nordeuropa ist zweifellos im 14. Jahrhundert völlig erloschen. Zu dieser Pestepidemie: MeIER, Justinianische Pest; LeVEn, Justinianische Pest.

${ }^{46} \mathrm{Zu}$ den Augsburger Chroniken im Spätmittelalter vgl. vor allem die Einleitungen der edierten Werke im Rahmen der Editionsreihe „Die Chroniken der deutschen Städte“, auch dazu WeBER, Geschichtsschreibung; JOACHIMSOHN, Geschichtsschreibung.

${ }^{47}$ JANKRIFT, Up dat god sich aver uns verbarmen wolde; HöHL, Pest; HAtJe, Pest in Basel; MARTIN, Pest in Würzburg; SCHMÖLzER, Pest in Wien; SCHÖPPLER, Pest in Regensburg; BÜHL, Pestepidemien in Nürnberg; FößEL, Der Schwarze Tod in Franken.
} 


\section{1. „Pestilentz“, „Brechen“ und „großes Sterben“ - Augsburger Quellenbegriffe für die Pest}

In den frühneuzeitlichen Chroniken von Achilles Gasser ${ }^{48}$ und Paul von Stetten ${ }^{49}$ wie auch in einem Flugblatt von 1629, das eine kurze Geschichte der Augsburger Pestepidemien und Friedhöfe darstellt ${ }^{50}$, finden sich Informationen über die „Pest“ bzw. „Pestilentz“ schon für die Jahre 987, 1042, 1092, 1098, 1103, 1220 und 1313. Diese Angaben sind aber eher als eine Rückprojektion der langen Erfahrung mit dem in der Frühneuzeit gut vertrauten Pestphänomen auf die mittelalterliche Zeitperiode als ein überzeugender Beleg für das in Augsburg so frühe Auftreten einer Epidemie derselben Art wie der „Schwarze Tod“ aus der Mitte des 14. Jahrhunderts zu bewerten. Man könnte in diesem Zusammenhang vermuten, dass die frühneuzeitlichen Verfasser auf heute nicht mehr vorhandene hochmittelalterliche Annalen und Chroniken oder auf deren spätmittelalterliche Abschriften von diesen zugreifen konnten, in denen Krankheiten mit hoher Mortalität allgemein mit dem lateinischen Begriff „pestilentia“ oder „pestis“ bezeichnet wurden ${ }^{51}$. Dieser Begriff bedeutete aber in der Semantik jener Zeit und vor dem Ausbruch des Schwarzen Todes lediglich allgemein „eine Seuche“ ${ }^{\text {“52. }}$. Auf solche Weise konnten vermeintliche Informationen über frühere Epidemien in die frühneuzeitlichen Chroniken aufgenommen und mit der Pest in der späteren zeitgenössischen Wahrnehmung gleichgestellt werden. Aufgrund derartiger Angaben ist es nicht möglich zu bestimmen, ob diese früheren Epidemien einen ähnlichen Charakter und Verlauf wie die Pest aus der Mitte des 14. Jahrhunderts hatten ${ }^{53}$. Die Erwähnung dieser Epidemien im Kontext von Hungersnöten kann möglicherweise auf das sogenannte Antoniusfeuer hinweisen, das in den früh- und hochmittelalterlichen Hungerszeiten eine häufige, durch die Vergiftung mit dem Mutterkorn-Pilz hervorgerufene Krankheit war ${ }^{54}$.

In der Mitte des 14. Jahrhunderts brach über Europa ein zuvor unbekanntes Seuchenphänomen herein, welches verheerende Auswirkungen auf die Sterberate der

\footnotetext{
${ }^{48}$ GASSER, Chronik, T. 2, S. 41, 52, 55-56, 74.

${ }^{49}$ STETTEN, Geschichte, IV. 8. 42, V. 8. 54, V. 18. 62, VI. 12.91.

${ }^{50}$ Christliche Erinnerung [Augsburg: Johannes Schultes 1629], HARMS, Flugblätter, Bd. 1, Nr. 236.

${ }^{51}$ Kaspar Augustin, der Verfasser des Flugblattes aus dem Jahr 1629 erwähnt, dass er seine Geschichte der Augsburger Pestepidemien anhand der näher nicht genannten Chroniken bearbeiten konnte: Sovil ich hab koennen erfarn/In bewehrten Cronicken gut, in: Christliche Erinnerung [Augsburg: Johannes Schultes 1629], HARMS, Flugblätter, Bd. 1, Nr. 236.

52 Du CANGe, Glossarium mediae et infimae Latinitatis, Bd. 1, S. 294; GeOrges, Ausführliches lateinischdeutsches Handwörterbuch, S. 1668-1669.

${ }^{53} \mathrm{Zu}$ den Angaben über das hochmittelalterliche Seuchengeschehen in westfälischen und rheinischen Städten in den chronikalischen Quellen und zu Schwierigkeiten ihrer Interpretationen in: JANKRIFT, Up dat god sich aver uns verbarmen wolde, S. 201-217.

${ }^{54}$ MisCHLEWSKI, Antoniusfeuer.
} 
europäischen Bevölkerung jener Zeit hatte. In der Forschung wird es häufig als der „Schwarze Tod“ bezeichnet. Diese Bezeichnung wurde aber von den mittelalterlichen Zeitgenossen nie verwendet $^{55}$. Der Begriff „Schwarzer Tod“ tauchte im Zusammenhang mit dieser spätmittelalterlichen Pandemie erstmals in der zweiten Hälfte des 16. Jahrhunderts in skandinavischen Chroniken auf. Das Adjektiv „Schwarz“ drückte vor allem einen existenziellen Schrecken aus, der durch diese Seuche unter den Zeitgenossen ausgelöst wurde. Gleichzeitig wies es auch auf die infolge von Blutungen unter der Haut entstehenden schwärzlichen Karbunkel hin, die als ein augenfälliges Symptom der Krankheit galten. Der Begriff „Schwarzer Tod“ verbreitete sich in einer weiteren Öffentlichkeit erst seit der ersten Hälfte des 19. Jahrhunderts, als er Eingang in die Forschungsliteratur fand ${ }^{56}$. Dazu trug vor allem der deutsche Arzt Justus Friedrich Karl Hecker bei, der 1832 unter dem Eindruck der damals wütenden Cholera die historische Abhandlung „Der Schwarze Tod im vierzehnten Jahrhundert" veröffentlichte ${ }^{57}$. Mit der mehrfach aufgelegten englischen Übersetzung seines Werkes $^{58}$ sowie durch das ein breites Lesepublikum erreichende Übersichtswerk zur englischen Geschichte von Elisabeth Cartwright Penrose aus dem Jahr $1859^{59}$ wurde der Terminus „Black Death“ auch im angelsächsischen Kulturkreis schnell popularisiert.

Im Zusammenhang damit stellt sich hier die Frage, mit welchen Begriffen dieses Seuchenphänomen von den Zeitgenossen bezeichnet wurde. Zu diesem Zweck werden alle Quellenbegriffe in der Augsburger Überlieferung analysiert, die in der damaligen Wahrnehmung für alle Epidemien derselben Art wie der „Schwarze Tod“ verwendet wurden. Dieses breite Begriffsspektrum lässt sich dabei in drei semantischen Gruppen einteilen.

Zur ersten Gruppe gehört die „Pestilentz“, die in verschiedenen lexikalischen Varianten aufgeschrieben wurde: leidige pestilent ${ }^{60}$, gefaehrliche sterbliche vergiftige pestilentz ${ }^{61}$, pestilentz leuffen ${ }^{62}$, beschwaerlich seücht pestilentz ${ }^{63}$, krankheit der pestilentz $^{64}$, sucht der pestilentz $^{65}$ oder pestilentzische seuch $^{66}$. Dieser Krankheitsbegriff wurde von gelehrten Medizinern schon in ihren ersten Traktaten gebraucht, in denen nach dem Ausbruch des

\footnotetext{
${ }^{55}$ HERLIHY, Der Schwarze Tod, S. 10.

${ }^{56}$ DERS., Der Schwarze Tod, S. 11; JANKRIFT, Up dat god sich aver uns verbarmen wolde, S. 8-9.

${ }^{57}$ HECKER, Der Schwarze Tod.

${ }^{58}$ HECKER, The Black Death.

${ }^{59}$ PENROSE, A History of England.

${ }^{60}$ GASSER, Chronik, T. 2, S. 158.

${ }^{61}$ StAA, CM IV, Nr. 24: Supplikation von Doktor Schludin an den Rat (aus dem Inhalt für 1535 datierbar).

${ }^{62}$ SStBA, $2^{\circ}$ Cod. Aug. 214, T. III, Supl. T. II., 1494: Vergleich zwischen dem Domkapitul und der Stadt Augspurg, die Aufrichtung eines neuen Gottesackers betreffend.

${ }^{63}$ MACHOCZEK, Der Reichstag zu Augsburg 1547/48, S. 206-210: Ordnung zu Augspurg im reichstag anno 1548 contra morbum pestilentialem per romanorem cesarem uffgericht anno etc. 1548, hier S. 207.

${ }^{64}$ StAA, CM IV, Nr. 7: Schreiben der vier Augsburger Stadtärzte (1521).

${ }^{65}$ StAA, Ratsprotokolle, 1547, Fol. 27.

${ }^{66}$ GASSER, Chronik, T. 3, S. 29.
} 
„Schwarzen Todes“ die damalige Pestlehre formuliert wurde ${ }^{67}$. Der lateinische Begriff „pestilentia“ oder „pestis“, mit dem man ursprünglich ganz allgemein eine Seuche bezeichnete, wurde seither ausschließlich für die Epidemie in der Mitte des 14. Jahrhunderts und die darauf folgenden Seuchen, welche nach damaliger Wahrnehmung identisch waren, verwendet.

Die zweite Gruppe von Begriffen, die ebenfalls von Augsburger Zeitgenossen häufig verwendet wurde, bilden brechen ${ }^{68}$, sucht des brechens ${ }^{69}$, sterbsucht an dem gebrechen ${ }^{70}$ und grozzen brechen ${ }^{71}$. Unter dem Wort „Brechen“ war ursprünglich eine Vielzahl von mit der Krankheit verbunden Phänomenen wie Mangel oder Schaden am Körper, Verwundung, Gebrechen, Kranksein oder Krankheit selbst verstanden worden $^{72}$. Dass dieser vorher allgemein verwendete Begriff zu einem gebräuchlichen Namen für die Pest umgedeutet wurde, könnte mit der Semantik des Verbes „durch-brechen“ zusammenhängen, mit dem das Vorkommen sichtbarer Krankheitserscheinungen auf dem Körper bezeichnet wurde. Diese Annahme bestätigen die autobiographischen Aufzeichnungen in der Chronik des Augsburger Bürgers Burkhard Zink. Als im Jahr 1438 die Seuche in der Stadt ausgebrochen war, erlag er selbst der Krankheit. Wie er über seine Erkrankung berichtete, hatte er damals den geprechen an zwaien enden, an dem hals und an dem bain bei den gemächten ${ }^{73}$. Darüber hinaus erwähnte er auch, dass 1418 sein Vater an dem gepresten der pestilenz gestorben war ${ }^{74}$. Mit dem Ausdruck „Brechen“ wurden daher von den Zeitgenossen anscheinend insbesondere die äußeren Pestzeichen, wie z. B. die Beulen, wahrgenommen ${ }^{75}$.

Als die dritte Gruppe von den in Augsburg geläufigen Pestbegriffen ist die Bezeichnung „großes Sterben“ zu nennen: sterbenden leuff ${ }^{76}$, sterben leuffen ${ }^{77}$, sterbent ${ }^{78}$, todslauffen $^{79}$,

\footnotetext{
${ }^{67}$ Zur Ätiologie, Prophylaxe und Therapie der Pest in der damaligen Medizinlehre, siehe die detaillierten Analysen in Kapitel IV und V.

${ }^{68}$ StChr, Bd. 5, S. 150.

${ }^{69}$ Augsburger gedruckte Pestordnung vom 12. August 1535, SStBA, $2^{\circ}$ S 14, Kapsel 1, Nr. 21.

${ }^{70}$ MACHOCZEK, Der Reichstag zu Augsburg 1547/48, S. 206-210: Ordnung zu Augspurg im reichstag anno 1548 contra morbum pestilentialem per romanorem cesarem uffgericht anno etc. 1548, hier S. 207.

${ }^{71}$ StChr, Bd. 4, S. 63.

${ }^{72}$ Dazu vgl. in: HöFLER, Krankheitsnamen-Buch, S. 71-72; FISCHER, Schwäbisches Wörterbuch, „Breche(n)““, Bd. 1, Sp. 1378-1379. Nach Matthias Lexer sind „prechen“, „brechen“, „gebrechen“ und „,brästen“, „,gebrästen“ mittelhochdeutsche Allgemeinbegriffe für Krankheit, in LEXER, Mittelhochdeutsches Handwörterbuch.

${ }^{73}$ StChr, Bd. 5, S. 137.

${ }^{74}$ Ebd. S. 135.

${ }^{75} \mathrm{Zu}$ den typischen Pestzeichen in der damaligen Medizinlehre, siehe S. 115-116.

${ }^{76}$ StAA, Ratsprotokolle, 1521, Fol. 10.

${ }^{77}$ StAA, Ratsprotokolle, 1521, Fol. 14.

${ }^{78}$ StChr, Bd. 23, S. 29.

${ }^{79}$ SStBA, $2^{\circ}$ Cod. Aug. 214, T. III, Supl. T. II., 1494: Vergleich zwischen dem Domkapitul und der Stadt Augspurg, die Aufrichtung eines neuen Gottesackers betreffend.
} 


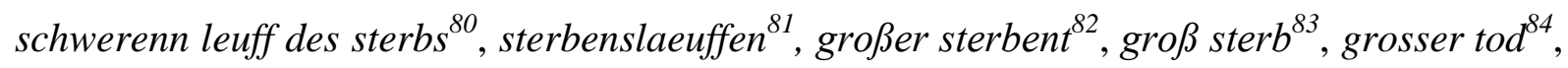
großer leut sterben ${ }^{85}$, grosser sterbet ${ }^{86}$ oder grosses sterben ${ }^{87}$. Mit diesem sprachlich am vielfältigsten fixierten Quellenausdruck wird primär ein krankheitsbedingtes Massensterben betont. Dadurch tritt der damalige Wahrnehmungsschwerpunkt dieser Krankheit zutage, der für die Zeitgenossen in der für jeden sichtbaren und rapide ansteigenden Mortalität der Bevölkerung lag.

In der Analyse der Augsburger Quellenbegriffe für die Pest ist festzustellen, dass „Pestilentz“, „Brechen“ und „großes Sterben“ von den Zeitgenossen synonym verwendet wurden. Der Begriff „Pestilentz“ wurde parallel zu anderen Ausdrücken gebraucht, so dass dieselbe Epidemie in einem Jahr mit vielen Namen gleichzeitig belegt wurde. Im gesamten Untersuchungszeitraum von der Mitte des 14. Jahrhunderts bis zum Jahr 1550 lässt sich auch kein eindeutiger Wandel im Gebrauch der oben genannten Quellenbegriffe beobachten. Eine beschränkte Differenzierung kann man aber aufgrund der Verwendung von „Pestilentz“, „Brechen“ und „großes Sterben“ im Hinblick auf die Quellengattungen konstatieren, die in der vorliegenden Studie ausgewertet wurden. Von den Ärzten wurde in erster Linie der Begriff „Pestilentz“ bevorzugt, der schon in allen Titeln ihrer medizinischen Pestschriften vorkommt $^{88}$. Die spätmittelalterlichen Chronisten berichteten über das „,große Sterben“, wobei in den frühneuzeitlichen Chroniken diese Bezeichnung überwiegend durch die "Pestilentz“ ersetzt wurde ${ }^{89}$. Diese Tatsache kann möglicherweise mit der sich damals vollziehenden Verbreitung der medizinischen Pestlehre in Augsburg zusammenhängen, in der die „Pestilentz“ als ein zentraler Begriff fungierte. Im Schriftgut des Augsburger Rates, das schrittweise mit der Entwicklung der obrigkeitlichen Pestpolitik um 1500 einsetzt, ist dagegen die gesamte Vielfalt der oben dargestellten Termini in ähnlicher Dichte anzutreffen. Dabei spielte aber der Begriff „Brechen“ in den städtischen Akten eine besondere Rolle. Die im Jahr 1521 erbaute Hospitalanlage für die Pestkranken bezeichneten die Ratsherren nämlich als „Brechhäuser، ${ }^{‘ 0}$. Damit wurde vor allem der Gründungszweck dieser städtischen Einrichtung

\footnotetext{
${ }^{80}$ SStBA, $2^{\circ}$ Cod. Aug. S 141, Fol. 118v.

${ }^{81}$ GASSER, Chronik, T. 2, S. 255.

${ }^{82}$ StChr, Bd. 23, S. 29.

${ }^{83}$ StChr, Bd. 5, S. 26.

${ }^{84}$ StChr, Bd. 4, S. 226.

${ }^{85}$ StChr, Bd. 22, S. 78.

${ }^{86}$ SStBA, $2^{\circ}$ Cod. Aug. 71, Fol. 35.

${ }^{87}$ StChr, Bd. 22, S. 463.

${ }^{88}$ Die einzige Ausnahme ist der Titel des Werkes von JOHANn KLAINMÜLLER (WOHLOPTER), Ain hymlischen vnn Natürlichen anzaygung dieser sterberlichen leüff...[Augsburg: Hans Schönsberger, 12. Oktober 1521].

${ }^{89}$ GAsser, Chronik, T. 2, S. 128, 135, 143, 146, 158, 173, 174, 207, 209; T. 3, S. 3, 29, 65; SStBA, $2^{\circ}$ Cod. Aug. 53, Fol. 167v; SStBA, $2^{\circ}$ Cod. Aug. 71, Fol. 188; SStBA, $2^{\circ}$ Cod. Aug. 80, Fol. 37.

${ }^{90}$ Zur Gründung der städtischen Brechhäuser, siehe S. 140-143.
} 
verdeutlicht, in der ausschließlich mit dem „Brechen“ befallene Kranken versorgt werden sollten.

Abschließend muss noch bemerkt werden, dass im gesamten Untersuchungszeitraum nur eine Epidemie mit enorm großer Mortalität von den Zeitgenossen nicht als Pest wahrgenommen und demnach auch mit einem anderen Namen bezeichnet wurde. Im September 1529 brach in Augsburg eine epidemische Krankheit aus, die innerhalb weniger Wochen bis zu 800 Todesopfer forderte. Diese ursprünglich in England ausgebrochene Seuche, die sich im Sommer 1529 auch in Deutschland blitzartig ausbreitete, wurde von den Zeitgenossen „Englischer Schweiß“ oder „Englische Schweißsucht“ genannt ${ }^{91}$. Nach der damaligen Wahrnehmung zeichnete sich diese rasch verlaufende Infektion vor allem durch starke Schweißausbrüche aus, die von sehr hohem Fieber begleitet wurden. Der Ausbruch dieses neuen Krankheitsphänomens im Jahr 1529 blieb in Augsburg wie auch in anderen deutschen Städten ein einmaliges Ereignis und wurde in späterer Zeit nicht mehr wahrgenommen bzw. notiert. Das Erscheinen dieser Seuche ist ein Beleg dafür, dass die Zeitgenossen in Augsburg mit den oben genannten Pestbegriffen alle damals auftretenden Epidemien bezeichneten und erst in der ersten Hälfte des 16. Jahrhunderts begannen diese von anderen Seuchen mit gleichfalls enorm großer Mortalität zu unterscheiden.

\section{Chronologie der Epidemien bis zum Jahr 1483}

Im Fall Augsburgs kann man annehmen, dass die Stadt von der ersten Pestwelle aus der Mitte des 14. Jahrhunderts weitgehend verschont blieb. In der Chronik von Gasser findet sich zwar die Erwähnung der Pest von 1348, die als eine Auswirkung des Erdbebens in Augsburg am 28. Januar 1347 entstanden sein soll, sowie eine Information über die darauf folgende Judenverfolgung 92 . Nach einer zeitgenössischen Pestdeutung wurde der „Schwarze Tod“ durch die angebliche Brunnenvergiftung von Juden verursacht, woraufhin es in ganz Europa

\footnotetext{
${ }^{91}$ RESCH, »Englischer Schweiß« 1529 in Augsburg; Allgemein zum „Englischen Schweiß“ in: PATRICK, A Consideration of the Nature; PÜschel, Der Englische Schweiß; THWAITES/TAVINER/Gant, The English Sweating Sickness.

${ }^{92}$ GASSER, Chronik, T. 2, S. 106: 1348: Ist jedoch die Statt Augspurg mit viel und mancherlay Plagen bedraengt gewesen. Dann nit allein den Sommer uber unsaglich viel Leuth an der Pestilentz gestorben welche man darfuer hielte das sie auß dem Erdbidem so den 28. Januarii des vorgehenden Jars viel Haeuser und Gebaew allhie eingeworffen und grossen schaden gethan entstanden were (...) Und seynd bald hernach nemlich den zwey und zwenzigsten Novembris viel Juden von Bischoff Hainrichen zum Fewer verdammt und verbrennet worden welchen man schuld geben daß sie in ganz Europa die Brunnen vergifftet: wie dann auch im nachfolgenden Jar an etlichen mehr Orthen viel ubel gepeiniget und hingerichtet worden.
} 
zu Judenpogromen $\mathrm{kam}^{93}$. Aufgrund einer detaillierten Analyse der Steuerbücher aus den Jahren 1346 und 1351 sowie weiterer zeitgenössischer Quellen bzw. Ereignisse konnte Raphael Krug jedoch nachweisen, dass der Schwarze Tod damals in Augsburg nicht auftrat ${ }^{94}$. Die fränkische Nachbarregion blieb von dieser europaweiten Katastrophe auch unberührt ${ }^{95}$. Das erste Auftreten der Pest in Augsburg ist daher erst in der zweiten Hälfte des 14. Jahrhunderts zu verorten.

In der Chronik von Clemens Sender (1475-1536), einem Mönch des St. Ulrich und Afra Klosters, wurde unter den Jahren 1358 und 1362 verzeichnet, dass in Augsburg ein großes sterbent war $^{96}$. Da Angaben über diese Pestgeschehnisse nur in dem Werk von Sender aus der ersten Hälfte des 16. Jahrhunderts aufgefunden wurden, scheint es auch möglich, dass die Pest in Augsburg zum ersten Mal in den Jahren 1379-1380 aufgetreten ist. Über diesen Pestzug berichten nämlich einige Chroniken, die zu diesem Pestgeschehen einen zeitgenössischen Bezug aufweisen oder nicht lange danach entstanden sind ${ }^{97}$.

Auf die Ungewöhnlichkeit und Besonderheit der hereinbrechenden Krankheit weist der Augsburger Kaufmann Burkhard Zink (1396-1474) in seiner Chronik hin, indem er feststellte, dass in Augsburg und überall auf dem Lande so ein großes sterben war, als sein ie gedacht warde $^{98}$. In diesem Zusammenhang schilderten die spätmittelalterlichen Chronisten ein düsteres Bild der Seuchenzeit, demzufolge in einigen Orten in der Umgebung Augsburgs sogar mehr als die Hälfte der Bevölkerung gestorben sei ${ }^{99}$. Auf den Feldern verblieb viel Getreide und der Mangel an Menschen war so gro $\beta$, dass nicht alle Äcker für das nächste Jahr bestellt werden konnten. Der Getreidepreis blieb aber stabil, da nur wenige Menschen überlebten, so dass die Nachfrage nach Lebensmitteln sogar deutlich abnahm. Die überlieferten Informationen über die verheerenden Pestfolgen in Augsburg und in seinem Umland zeigen aus heutiger Perspektive vor allem einen mächtigen Überraschungseffekt und die große Unvorbereitetheit der Zeitgenossen auf ein solches Ereignis, aus dem sich eine beträchtliche Betroffenheit der ganzen Stadtgemeinschaft ergeben konnte.

\footnotetext{
${ }^{93}$ Zur Judenverfolgung im Europa während des Schwarzen Todes vgl. in: CoHN, The Black Death; GRAUS, Pest; JÄCKEL, Judenmord; RITZMANN, Judenmord.

${ }^{94}$ KRUG, Augsburger Steuerbücher, S. 144-146.

${ }^{95}$ FÖßEL, Schwarze Tod in Franken, S. 17f.

${ }^{96}$ StChr, Bd. 23, S. 29.

${ }^{97}$ StChr, Bd. 4, S. 63, 66-67, 226, 313; Bd. 5, S. 26; Bd. 22, S. 24.

${ }^{98} \mathrm{StChr}, \mathrm{Bd} .5$, S. 26. In den frühneuzeitlichen Chroniken wird berichtet, dass damals in Augsburg die Hälfte aller Bevölkerung gestorben sei: in SStBA, $2^{\circ}$ Cod. Aug. 80, Fol. 37: starb an solcher Pest fast der halbe theil der Burgerschaft alhie; GASSER, Chronik, T. 2, S. 128: daran auch fast die Helffte der Burgerschaft ja auch ein grosse anzahl Viehes auff dem lauff geblieben.

${ }^{99}$ StChr, Bd. 4, S. 66-67, Bd. 5, S. 26.
} 
In den chronikalischen Berichten wird diese Seuchenzeit als ein traumatisches Erlebnis geschildert: dann es ward ain ellend erschrocken ding, es möchte schier alles volk verzweifelt $h a n^{100}$ und daz die lüt unbesint würden von dem bösen gaist ${ }^{101}$. Um diesem grauenhaften Krankheitsphänomen einen tieferen Sinn zu verleihen und die mentale Bewältigung der Ängste in der Pestzeit zu ermöglichen, griffen die Zeitgenossen auf ein im Mittelalter bewährtes und allgemeingültiges Deutungsmuster in Krisenzeiten zurück: die Religion ${ }^{102}$. In der Erscheinung einer Krankheit sah man die übernatürliche Wirkungskraft Gottes schlechthin und verankerte ihren Ursprung im religiösen Kontext. Ein Pestausbruch wurde daher von den Menschen als eine göttliche Strafe für begangene Sünden wahrgenommen. Um den Zorn Gottes besänftigen zu können, musste man mit Hilfe verschiedener religiöser Handlungen um göttliche Gnade bitten. Dies allein versprach die Aussicht auf eine Abwendung der Krankheit. Zum üblichen Maßnahmenkatalog der religiösen Bewältigung der Pest gehörten im Spätmittelalter Bittprozessionen, Votivmessen, Fastenzeiten, Gebete zu Schutzpatronen wie dem Heiligen Sebastian oder dem Heiligen Rochus, wie auch die Errichtung verschiedenartiger Stiftungen für die Kirche.

Derartige fromme Handlungen sind auch während des ersten Pestzuges in Augsburg zu beobachten ${ }^{103}$. Am 4. Juni 1379 veranstalteten die Augsburger Priester die erste Bittprozession mit Herumtragen des Heiligen Sakraments und aller Reliquien um die Stadt, die von Mönchen, Schülern und allen Stadteinwohnern begleitet wurde. Mit großer Andacht sangen sie vor jedem Stadttor die Evangelientexte und erbaten von Gott und der Jungfrau Maria die gnädige Abwendung des sterbens ${ }^{104}$. Die nächste Bittprozession in Augsburg ist ein Jahr später, am 15. September 1380, bezeugt ${ }^{105}$.

Den frühneuzeitlichen Chroniken zufolge begehrten alle Stadtbewohner während dieser Pestepidemie unzählige Seelenmessen und Glockengeläute für Verstorbene zu stiften, wie auch in zahlreichen Trauerzügen ihrer Frömmigkeit einen deutlichen Ausdruck zu geben ${ }^{106}$. Zu diesem Zweck vermachten die Menschen in ihren Testamenten viel Vermögen der Kirche, um dadurch sich selbst und ihren Familien das Seelenheil zu sichern. Wegen der enormen Stiftungsbereitschaft der Bevölkerung erließ der Rat aber eine Verordnung, in welcher

\footnotetext{
${ }^{100}$ StChr, Bd. 5, S. 26.

${ }^{101}$ StChr, Bd. 4, S. 63.

${ }^{102}$ In der Forschung wurden die Grundlinien der religiösen Pestbewältigung im Spätmittelalter in vielen Arbeiten ausführlich erforscht, deswegen wird diese Frage hier auf das Wichtigste beschränkt. Zu diesem Thema in erster Linie: ESSER, Pest; BULST, Heiligenverehrung; DERS. Krankheit; DORMEIER, Schutzpatrone; DERS.: Laienfrömmigkeit; TRÜB, Heilige.

${ }^{103}$ StChr, Bd. 4, S. 63, 66-67, 313; Bd. 5, S. 26; Bd. 22, S. 24.

${ }^{104}$ StChr, Bd. 4, S. 63.

${ }^{105}$ StChr, Bd. 4, S. 66-67.

${ }^{106}$ SStBA, $2^{\circ}$ Cod. Aug. 80, Fol. 37; GASSER, Chronik, T. 2, S. 128.
} 
angemahnt wurde, dass man sich in der Ausübung solcher frommen Praktiken mäßigen solle. In der Chronik von Gasser wurde die Ermahnung des Rates zur Einschränkung der bürgerlichen Stiftungen mit folgender Erklärung kommentiert: damit solches ohne der Erben schaden und abbruch des gemeinen Nutzens geschehe ${ }^{107}$. Neben der Sicherung der reichlichen Erbgüter für Nachkommen konnte der Rat mit einer solchen Verordnung vor allem die Absicht verfolgen, die möglichst große Steuerpflichtigkeit aller Bürger zu erhalten und der Übertragung von Grund und Boden in der Stadt durch Schenkung oder Verkauf an die Kirche entgegenzuwirken. Wenn bürgerliches Vermögen in großen Mengen an die sogenannte „Tote Hand“" der Kirche überging, fiel es dadurch aus der städtischen Besteuerung heraus ${ }^{108}$.

Kurz nach diesem wahrscheinlich ersten Pestausbruch in Augsburg folgten die nächsten Epidemien in den Jahren $1389^{109}$ und $1398^{110}$. Auch für diesen Zeitraum sind ganztägige Prozessionen bezeugt, während denen das Evangelium vor jedem Stadttor vorgelesen wurde. Das Auftreten der Pestepidemie im Jahr 1402, die insgesamt eine Zahl von 4650 Toten verursacht haben soll, ist nicht gesichert, weil nur die frühneuzeitlichen Chroniken das Ereignis überliefern ${ }^{111}$. Dagegen wurde die nächste Pestepidemie 1407 in mehreren Chroniken kurz vermerkt ${ }^{112}$.

Im Jahr 1420 grassierte die Pest erneut in Augsburg ${ }^{113}$. Die Mortalität in der Stadt und überall auf dem Lande sei erneut so stark gewesen, dass nach Mitteilung der Chronisten die Kornfelder in den umliegenden Dörfern nicht abgeerntet wurden. Die dabei angegebene Anzahl von 16.000 Pestopfern kann aber keineswegs die realen demographischen Auswirkungen während der Pestzeit wiedergeben. Nach den Studien von Joachim Jahn zur demographischen Entwicklung wuchs die Augsburger Bevölkerung von 1396 bis 1492 von rund 12.000 auf etwa $19.000 \mathrm{an}^{114}$. Wenn tatsächlich ca. 16.000 Stadteinwohner von der Epidemie dahingerafft worden wären, hätte Augsburg anschließend fast menschenleer sein müssen. Aus diesem Grund kann man die Anzahl von 16.000 Pestopfern wie auch die anderen Angaben über die Pestopferzahlen in den spätmittelalterlichen Chroniken nur mit Vorsicht bewerten. Die chronikalischen Berichte über die verheerenden Epidemiefolgen sollten vor

\footnotetext{
${ }^{107}$ GASSER, Chronik, T. 2, S. 128.

${ }^{108}$ Vgl. KIESSLING, Bürgertum, S. 208f.

${ }^{109}$ StChr, Bd. 4, S. 228; SStBA, $2^{\circ}$ Cod. Aug. 71, Fol. 26.

${ }^{110}$ StChr, Bd. 4, S. 228; Bd. 22, S. 463.

${ }^{111}$ STETTEN, Chronik, VII. 12. 140; GASSER, Chronik, T. 2, S. 143.

${ }^{112}$ StChr, Bd. 22, S. 53, S. 465; Bd. 4, S. 229; SStBA, $2^{\circ}$ Cod. Aug. 71, Fol. 35; GASSER, Chronik, T. 2 , S. 146.

${ }^{113}$ Hier ist insbesondere der Bericht von Burkhard Zink interessant: StChr, Bd. 5, S. 68-69, 129-130, 148; dazu auch in StChr, Bd. 22, S. 64; Bd. 4, S. 119, 232; GASSER, Chronik, T. 2, S. 158; STETTEN, Chronik, VII. 18.149. Dieser Pestzug wurde auch unter dem Jahr 1419 vermerkt, in: StChr, Bd. 22, S. 64, 477.

${ }^{114}$ JAHN, Sozialstruktur, S. 188; auch DERS., Einwohnerzahl.
} 
allem als anschauliche Hervorhebung der enormen Sterblichkeit in der Pestzeit und nicht als eine exakte Wiedergabe der Opferzahlen angesehen werden.

Über den Verlauf der Pestepidemie im Jahr 1420 sind einige interessante Details in den Aufzeichnungen von Burkhard Zink überliefert, der dieses Ereignis in Augsburg selbst miterlebte. Während der Epidemie zogen sich viele Bürger aus der Stadt zurück, als man dann tuet, dann iedermann wolt geren leben ${ }^{115}$. Trotz der unternommenen Pestflucht starben aber einige Augsburger in den benachbarten Orten und wurden sodann zur Bestattung wieder in die Stadt zurückgebracht. So wurde auch die Leiche eines Bürgers namens Püttinger, der während der Pestzeit in Donauwörth gestorben war, auf dem St. Maria Kirchhof am Dom nach christlicher Ordnung begraben. Kurze Zeit danach erließ der Augsburger Bischof Anshelm von Nenningen ein Interdikt, dass man Püttinger wieder ausgraben sollte. Diese Anordnung war damit begründet, dass Püttinger ohne Gottes Recht und Beichte in Donauwörth gestorben und in seinem ganzen Leben ein rechter pfaffenfeind gewesen sei. Die Augsburger Priester waren bereit, dem bischöflichen Willen entgegenzukommen und die Grabstätte von Püttinger außerhalb des Kirchhofs zu verlegen. Der städtische Rat war aber damit nicht einverstanden und verbot die Ausführung der bischöflichen Anordnung. Ob dem Verhalten des Rates eine allgemeine Sorge um die Reinhaltung des öffentlichen Raumes in der Pestzeit zugrunde lag, lässt sich eher bezweifeln. Als sicher gilt aber, dass der Rat sich seit 1413 mit dem Augsburger Bischof in einem heftigen Streit befand und sich mit aller Kraft bemühte, Anshelm von Nenningen vom Bischofsamt abzusetzen ${ }^{116}$. Aus diesem Grund kann der Konflikt von 1420 eher als eine weitere Machtprobe zwischen der städtischen und bischöflichen Autorität erklärt werden. Im Gegenzug befahl der Bischof die Spendung der Sakramente in der Stadt einzustellen. Unter Androhung des bischöflichen Banns hörten die Priester in allen Pfarreien auf, Messen zu lesen und Verstorbene zu bestatten. Diese Art des kirchlichen Widerstandes während des Bischofsstreites war nicht neu und wurde zum ersten Mal 1418 als Druckmittel gegen die städtische Obrigkeit eingesetzt ${ }^{117}$.

Der Rat versuchte unter Vermittlung von Juristen mit dem Propst der St. Georg-Kirche zu verhandeln, um alle Verstorbenen vorübergehend auf seinem Kirchhof bestatten zu können. Darüber hinaus wollten die Ratsherren künftig in jeder Pfarre einen neuen Priester anstellen, um dadurch die sakrale Versorgung der Verstorbenen in der Stadt wieder in Gang zu setzen. Der Propst von St. Georg ging aber aus Angst vor dem bischöflichen Bann nicht näher auf

\footnotetext{
${ }^{115}$ StChr, Bd. 5, S. 68-69.

116 Zum Streit zwischen dem Augsburger Rat und dem Bischof Anshelm von Nenningen (1413-1423) in: ZoEPFL, Bischöfe im Mittelalter, S. 360-380.

${ }^{117}$ Ebd. S. 368; StChr, Bd. 22, S. 471.
} 
den Vorschlag des Rates ein. Dagegen konnte die städtische Obrigkeit nichts mehr unternehmen und gab ihren Widerstand gegen die umstrittene Exhumierung auf. Die Leiche Püttingers wurde nun endgültig ausgegraben und auf einen Platz am Turm der St. GallusKirche an der Stadtmauer beigesetzt. Danach nahmen die Priester ihre religiösen Pflichten wieder auf. Der Streit zwischen dem Rat und dem Bischof dauerte insgesamt 14 Tage. Trotz der ausgebrochenen Pestepidemie wurde während der Streitdauer niemand in Augsburg begraben und waren wol 80 mentschen tot in allen pfarren, die stuenden alle noch ob der erden in truchen ${ }^{118}$.

Seit dem Herbst $1429^{119}$ regierte die Pest wieder in Augsburg und dauerte bis zum Beginn des Jahres $1431^{120}$. Nach den chronikalischen Angaben starben in dieser Zeit 6.000 Menschen in Augsburg. Dieselbe Anzahl von Pestopfern verzeichneten die Chroniken auch für die nächste Pestepidemie, die in den Jahren 1438-1439 regierte $^{121}$. Viele Verstorbene wurden damals in einer großen Grube an der Domkirche neben der St. Johannes Tür begraben. Ähnlich wie im Jahr 1420 zogen sich die reichen Bürger aus der Stadt zurück. Trotz der unternommenen Pestflucht in die Nachbarorte starben viele von ihnen auch dieses Mal und wurden dann nach Augsburg zur Bestattung zurückgebracht. Ungeachtet ihrer städtischen Pflichten verließen in der Pestzeit auch die Ratsherren und Bürgermeister die Stadt. Im Jahr 1438 scheint sich aber die Überzeugung durchzusetzen, dass sich nicht alle Amtsträger während der Epidemie aus der Stadt zurückziehen sollten. Zu diesem Zweck bestimmte der Rat eine spezielle Sitzungsordnung, die jeden Ratsherrn verpflichtete, 14 Tage lang das Bürgermeisteramt $\mathrm{zu}$ bekleiden. Wer außerhalb der Stadt verweilte, musste bei Strafandrohung während seiner Amtszeit wieder nach Augsburg zurückkommen ${ }^{122}$.

Über die nächste Pestepidemie in den Jahren 1462-1463 berichtet Burghard Zink, der während der Seuche auch diesmal in Augsburg anwesend war, wieder ausführlicher ${ }^{123}$. Nach seinen Schilderungen gingen dem Pestaubruch von 1462 zwei andere Krankheiten voraus:

\footnotetext{
${ }^{118}$ StChr, Bd. 5, S. 69.

${ }^{119}$ Ebd. S. 149: Auf den Beginn der Epidemie bereits Ende des Jahres 1429 deutet ein Schreiben des Augsburger Rates vom 28. November 1429 hin, worin dieser die Bitte der Ulmer ihnen eine Ratsbotschaft zu leihen, abschlagen musste, „auf die Zeit Brechen in unseren Rätern haben, das die nicht anheim und von wegen der Pestilentz aus unserer Stadt geflohen sind“".

${ }^{120}$ StChr, Bd. 4, S. 232; Bd. 5, S. 136, 149; GASSER, Chronik, T. 2, S. 168.

${ }^{121}$ StChr, Bd. 5, S. 137; Bd. 22, S. 78, 488-489; Bd. 4, S. 323. Die Epidemie begann am 25. Juli 1438.

${ }^{122}$ In GASSER, Chronik, T. 2, S. 173 wird dagegen berichtet, daß alle Vierteljar ein jeder deß Raths der Ordnung nach, wie sie im Rath zusitzen pflegten, Burgermaister ward, unnd der dritte theil der Herrn zu verwesung der gewoehnlichen Aemter, zu gewissen Taegen, in die Statt kommen mussten, Gott geb, wo sie sich gleich hielten, und solches an Eydts statt angeloben.

${ }^{123}$ StChr, Bd. 5, S. 293-295. Außerdem auch allgemeine Informationen über die Pest in 1462-1463 in: StChr, Bd. 4, S. 330; Bd. 22, S. 197-198.
} 
eine allgemeine grippenartige Krankheit, genannt ,pürtzel“124, und eine Durchfallerkrankung, die als „rote Ruhr"125 bezeichnet wurde. Daran starben nur einige Leute und die Erkrankungen verliefen in den meisten Fällen relativ harmlos. Kurz darauf brach aber erneut die Pest in Augsburg aus, die dann bis Ende 1463 währte. Nach Zinks Schilderungen verhielten sich die meisten Bürger im Anfangsstadium der Epidemie aber eher unbesorgt: wiewol vil leut fast krank waren und sturben, so kümeret doch solchs wenig leut oder niemant: man tanzet, man hochzeitet und waren die leut frölich; wiewol doch ie vil leut sturben, so gab niemant nichts darumb, dann wem geschah, der muest schaden han ${ }^{126}$.

Der Augsburger Alltag in der Pestzeit verlief also anfangs noch völlig normal: Niemand zog sich aus der Stadt zurück oder verhielt sich frömmer als zuvor. Im Laufe der Zeit starben aber immer mehr Leute in der Stadt: nun starb es ie lenger ie fester und was im niemant weder zu alt noch zu jung: es sturben man und frawen, die bei 60 jarn weder zu alt noch zu jung, aber es sturben dennocht mer jung dann alter ${ }^{127}$. Erst als Ende Juli 1463 die Menschen massenhaft von der Seuche hinweggerafft worden waren, begannen viele reiche Bürger aus der Stadt zu fliehen. Zink verzeichnete darüber hinaus auch viele aufschlussreiche Informationen über die Organisation des Begräbniswesens in der Pestzeit. Für die armen Leute, die sich keine eigenen Gräber auf dem Friedhof leisten konnten, wurden Gemeinschaftsgräber angelegt, in denen alle Verstorbenen zusammen bestattet wurden. Während dieser Pestepidemie von 1462 bis 1463 beerdigte man die Armen in insgesamt neun Gruben auf den innerstädtischen Kirchhöfen. Von denen befanden sich fünf auf dem St. Ulrich Kirchhof, drei am Dom und eine bei der St. Georg Kirche. Zwei der größten Gruben wurden auch innerhalb der Stadtmauer angelegt, die eine am Dom zwischen der St. Johannes Kirche und dem Fronhof und die andere bei der St. Ulrich Kirche neben dem turn bei der schuel fenster. Sie waren entsprechend bei 20 schuechen weit auf all ort und drei mann tief und 24 schuech weit auf

\footnotetext{
${ }^{124}$ Zur zeitgenössischen Krankheitsbezeichnung „Pürtzel/Bürtzel“ vgl. in: HöFLER, Krankheitsnamen-Buch, S. 81-82; StChr, Bd. 5, S. 293: da kam ain gemain plag und pürtzel under die leut, dem was also: die leut wurden krank und ward in den köpfen wee, auch gewunnen etlich mentschen grimen in dem leib und wurden etlich leut unbesint, und sturben doch nit alle, ir genas der mertail und wurden wider gesunt. Über dieselbe Krankheit berichtet noch Zink für das Jahr 1387, Ebd. S. 32: In dem jar 1387 do kam ain großer pörtzel in alle land und stett under die leut, und wurden gar vil menschen krank und lagen 3 oder 4 tag und stuenden dann wider auf, und für das Jahr 1466, Ebd. S. 312: Item ist zu wißen, daß in dem obgemanten jar anno 66 was ain gemainer pörtzel hie in der stat und auch anderstwa weit und prait under den jungen kinden. die huesteten alle so ser, daß alles das von in prach, das in in was (mit urlaub, salve reverentia), sie huesteten oft und dick, daß aiter, pluet und rotz von in prach, und sturben vil kind an dem huesten, also daß sie erstickten. und hueb sich der pörtzel an an dem herbst nach sant Bartholomei tag anno 66 [24. August].

${ }^{125}$ Bei der „roten Ruhr“ handelt es sich vermutlich um eine dysenterieartige Krankheit, die nach dem Auftreten von heftigen Darmschmerzen vor allem durch blutschleimige Stuhlentleerungen gekennzeichnet ist, vgl. in: HÖFLER, Krankheitsnamen-Buch, S. 530-531; StChr, Bd. 5, S. 293: auch kamen gar vil leut die rot ruer an und mochten auch gar übel daran und sturben etlich und genasen auch etlich.

${ }^{126}$ StChr, Bd. 5, S. 293.

${ }^{127}$ Ebd.
} 
baid ört und wol so tief ${ }^{128}$. In den kleineren Massengräbern wurden etwa 15 bis 200 Pestopfer auf einmal begraben. Dagegen war in den zwei größten Gruben die Anzahl der Bestatteten sicher beträchtlich höher.

In der Zeit vom 16. Oktober 1462 bis zum 29. September 1463 fielen nach Zink insgesamt 10.000 Menschen der Pest zum Opfer. Danach starben bis zum Ende 1463 nur wenige Leute in der Stadt, so dass man in allen Augsburger Pfarreien nur drei bis vier Tote pro Tag registrierte. Zink berichtet auch darüber, wie diese Todesstatistik, von der er seinen Angaben zufolge, von einem frommen reichen Mann erfuhr, zusammengestellt wurde. Ein Augsburger Bürger verordnete nämlich ain redlichen man in allen pfarren und lassen mit vlei $\beta$ fragen, wie vil leut da gestorben weren ${ }^{129}$. Nach seiner Rechnung belief sich die Gesamtzahl der Toten von 1462 bis 1463 auf 11.000. Diese Opferzählung während der Pestzeit wurde also nicht vom Rat angeordnet und ist vollständig auf eine Privatinitiative zurückzuführen.

Dagegen schätzte Hektor Müllich, ein Augsburger Kaufmann und Ratsmitglied, in seiner Chronik, dass vom 25. Juli bis zum 13. Dezember 1463 ungefähr 9.000 Menschen gestorben sind $^{130}$. Nur in der St. Ulrich Pfarrei seien 3.500 Personen verstorben. Müllich vermerkte ebenfalls, dass in der Pestzeit viele Leute aus der Stadt flohen, deren Anzahl seiner Ansicht nach ca. 3.000 Menschen betrug. Er selbst fuhr mit seiner Familie am 11. August 1463 nach Schwabmünchen. Am 6. Dezember 1463 zog er dann nach Memmingen um, von wo er am 6. Januar 1464 nach Augsburg zurückkehrte. Seit dem 29. September 1463 verweilte Müllich insgesamt nur vier Tage in Augsburg, in denen nach seiner Schätzung circa 500 Menschen ums Leben kamen. Obwohl die in den Chroniken angegeben Peststatistiken auf zeitgenössischen Beobachtungen oder auch auf eigens durchgeführten Opferzählungen beruhen, muss man jedoch den hohen Todeszahlen von 1462-1463 mit Vorsicht begegnen. Es kann aber als gesichert gelten, dass sich in diesen vermutlich übertriebenen Schätzungen eine generelle Pestvorstellung der Zeitgenossen im Spätmittelalter widerspiegelt: die Pest wurde vor allem als ein großes sterben wahrgenommen.

Am 20. August 1466 traf der Augsburger Bischof die Verfügung, Gott um den Sieg über die Christenfeinde und die Abwendung der herrschenden Pest und Teuerung zu bitten ${ }^{131}$. In seiner Diözese sollte nämlich bis auf Widerruf am ersten Tag jedes Monats ein Bittgang von jeder Pfarrei stattfinden und zusätzlich an diesem Tag eine Fastenzeit gehalten werden. Darüber hinaus wurden an jedem Freitag Prozessionen von den Kloster-, Stifts- und

\footnotetext{
${ }^{128}$ Ebd. S. 293-294.

${ }^{129}$ Ebd. S. 295.

${ }^{130}$ StChr, Bd. 22, S. 197-198.

${ }^{131}$ BRAUN, Geschichte, Bd. 4, S. 51.
} 
Pfarrkirchen veranstaltet. Allen Gläubigen, die diesen Andachten beiwohnten, erlaubte der Bischof, Ablässe zu erteilen. Die Pest in Augsburg dauerte bis zum Jahr 1467 an, worüber eine kurze Angabe in der Chronik von Gasser informiert ${ }^{132}$.

Über die zwei nächsten Pestepidemien in Augsburg lassen sich außer den allgemeinen Angaben keine Details finden. Für das Jahr 1473 erwähnt Paul von Stetten eine Pestseuche in der Reichstadt ${ }^{133}$. Nach der Chronik von Clemens Sender regierte die Pest auch 1483 im ganzen Schwabenland ${ }^{134}$. Ob Augsburg ebenfalls davon betroffen war, kann nicht mit Gewissheit gesagt werden ${ }^{135}$.

\section{Grundzüge der Pestwahrnehmung und -bewältigung im Spätmittelalter}

Aufgrund der chronikalischen Aufzeichnungen lässt sich trotz ihrer zumeist kurzen Darstellungsform und allgemeinen Aussagekraft ein generelles Bild der Pestwahrnehmung und -bewältigung im spätmittelalterlichen Augsburg zusammenstellen. Zum Ersten fällt die fundamentale Rolle des religiösen Diskurses auf, der die zeitgenössische Wahrnehmung damals am stärksten steuerte. Die Pest wurde im Spätmittelalter vor allem als übernatürliche Wirkungskraft Gottes erklärt, die nur durch den Einsatz religiöser Mittel abgewendet werden konnte. Die zu diesem Zweck verrichteten Handlungen kann man sowohl auf der städtischen wie auch auf der individuellen Ebene konstatieren, was auf die allgemeine Geltung dieser Praktiken in der Augsburger Stadtgemeinde hinweist. Die Veranstaltung von Bittprozessionen verbunden mit dem Zeigen und Herumtragen aller Heiltümer um die Stadt, die Lesung des Evangeliums vor den Stadttoren, die Zelebrierung sogenannter Pestmessen ${ }^{136}$ oder die Errichtung frommer Stiftungen durch die Bürger bestätigen die fundamentale Bedeutung der religiösen Pestbewältigung. Eine religiöse Ausprägung der individuellen Pestwahrnehmung ist auch in den autobiographischen Aufzeichnungen von Burghard Zink festzustellen ${ }^{137}$. Während seiner Pesterkrankung empfing er die heiligen Sakramente auf dem Krankenbett und sah es ausschließlich als eine barmherzige Fügung Gottes an, dass er und seine schwangere Frau wieder gesund wurden. Auch seine gottesfürchtigen Ausrufe im Kontext des

\footnotetext{
${ }^{132}$ GASSER, Chronik, T. 2, S. 216.

${ }^{133}$ STETTEN, Chronik, VII. 43. 212.

${ }^{134}$ StChr, Bd. 23, S. 43.

135 Auf das Auftreten der Pest in Augsburg in den Jahren 1473 und 1483 können auch die Ausgaben von Pestdrucken in dieser Zeit hinweisen, siehe S. 51 und 58-59.

${ }^{136}$ Ein lateinischer Text der Pestmesse Recordare aus dem letzten Jahrzehnt des 15. Jahrhunderts in der Diözese Augsburg ediert in: ESSER, Pest, S. 363-389.

${ }^{137}$ StChr, Bd. 5, S. 137.
} 
Pestgeschehens in Augsburg: gott herr kom uns zu hilf oder herr gott erbarm dich über sie all und bis in gnedig durch dein gruntlosen parmhertzigkait bestätigen eindeutig seine religiöse Deutung des Pestphänomens ${ }^{138}$.

Zum anderen prägte die direkte körperliche Erfahrung mit den biologischen Auswirkungen der Krankheit das spätmittelalterliche Bild des Pestphänomens. Die Zeitgenossen nahmen es vor allem als „großes Sterben“ wahr, wodurch die übliche Vorstellung der Pest als einer Krankheit mit sehr hohen Todeszahlen anschaulich zum Ausdruck gebracht wird. Die enorme Virulenz der Pest konstituierte ihre zeitgenössische Wahrnehmung und bestimmte zugleich den wichtigsten Unterschied $\mathrm{zu}$ anderen im Spätmittelalter auftretenden Krankheiten. $\mathrm{Zu}$ diesem Zweck wurden in den Chroniken die aus heutiger Sicht bestürzend wirkenden Todesstatistiken für die Pestzeit verzeichnet, um dadurch vor allem die ungewöhnlich hohe Sterblichkeit hervorzuheben.

Den autobiographischen Aufzeichnungen von Burghard Zink ist auch zu entnehmen, dass die Zeitgenossen während ihres Lebens mehrfach mit Pestepidemien konfrontiert werden konnten ${ }^{139}$. Zum ersten Mal war die Familie von Burghard Zink im Jahr 1418 davon betroffen, als sein Vater und seine Schwester in Memmingen an der pestilentz starben. Während des nächsten Pestzuges 1430-1431 erlagen der Seuche zwei seiner Töchter, Anna und Dorothea. Im Jahr 1438 starb auch sein Sohn Konrad. Burghard Zink erkrankte damals ebenfalls an der Pest, allerdings konnte er sich von der Krankheit erholen. Dagegen wurde seine Familie während der Epidemie in den Jahren 1462-1463 von der Pest verschont. Das Beispiel der Familie Zink zeigt deutlich, dass die Pest als ein wiederkehrendes Krankheitsphänomen von den Zeitgenossen wahrgenommen wurde, deren erneutes Auftreten immer mit höchster Gefahr für Leib und Leben verbunden war.

Zum Dritten lässt sich feststellen, dass die Flucht angesichts der Pestbedrohung eine wichtige Handlungsoption war. Während der Pestzeiten erkannten die Zeitgenossen aufgrund eigener Beobachtungen und Erfahrungen, dass der Aufenthalt an einem verpesteten Ort der eigenen Erkrankung deutlichen Vorschub leisten konnte. Aufgrund dieser Erkenntnis zogen sich die Menschen aus der Stadt in die pestfreie Nachbarschaft zurück. Die Stadt konnten jedoch nur solche Bürger verlassen, die über die dazu nötigen sozial-finanziellen Ressourcen verfügten.

Die Möglichkeit der Pestflucht beanspruchten u.a. die Stadtherren bis 1438 völlig unbeschränkt, d.h. ungeachtet ihrer Amtspflichten konnten sie Augsburg für längere Zeit verlassen. Vor diesem Hintergrund ist anzunehmen, dass die Bewältigung der Pest im

\footnotetext{
${ }^{138}$ Ebd. S. 294-295.

${ }^{139}$ Ebd. S. 135-137, 149-150, 295.
} 
spätmittelalterlichen Augsburg nur in bescheidenem Ausmaß von der Obrigkeit verwaltet werden konnte ${ }^{140}$. Die überlieferten Quellenbelege über die Aktivität der Stadtoberen beziehen sich ausschließlich auf die Bestattung von Pestopfern. Der Rat bemühte sich nämlich vor allem für die armen Stadtbewohner möglichst viele Gemeinschaftsgruben auf den Kirchhöfen innerhalb der Stadt zur Verfügung zu stellen. Aber nicht alles verlief reibungslos. Wie der Konflikt mit dem Augsburger Bischof Anshelm von Nenningen im Jahr 1420 deutlich zeigt, konnten solche städtischen Maßnahmen z.B. aufgrund des Widerstands der Kirche zumindest temporär verhindert werden. Dieser Vorfall enthüllt einerseits die beschränkten Handlungsmöglichkeiten der städtischen Obrigkeit während der Pestzeit, andererseits bestätigt er die Vorherrschaft des religiösen Pestdiskurses im Spätmittelalter, dessen mächtiger Träger die Kirche war. Außerhalb des Begräbniswesens findet man keinerlei Informationen mehr über andere Betätigungsfelder des Rates im Zusammenhang mit den Pestepidemien. Dies ist ein Hinweis auf die noch gering ausgebildete Professionalität der städtischen Pestpolitik.

\section{Das städtische Medizinalwesen und der medizinische Diskurs in der Pestzeit}

In den Augsburger Chroniken sucht man auch vergebens nach Hinweisen auf die Rolle der Mediziner während der spätmittelalterlichen Epidemien. Dass medizinische Praktiken hier keine Erwähnung fanden, mag vor allem aus der Eigenart der chronikalischen Quellen resultieren und muss nicht zwangsläufig ein Indiz für eine fehlende Relevanz des medizinischen Diskurses im Spätmittelalter sein. Für eine gewisse Bedeutung dieses Diskurses spricht die Tatsache, dass sich im spätmittelalterlichen Augsburg die grundlegenden Strukturen des städtischen Medizinalwesens entwickeln konnten ${ }^{141}$. In der Stadt betätigten sich vor allem zahlreiche Bader, Wundärzte und Barbiere ${ }^{142}$. Diese drei Medizinergruppen waren $\mathrm{zu}$ einem Handwerk vereinigt, wobei ihre interne Ausdifferenzierung noch bis weit in das 16. Jahrhundert hinein nicht konkret festgelegt war. $\mathrm{Zu}$ ihren wichtigsten Aufgaben gehörten die Behandlung äußerlich sichtbarer Krankheiten und das Verbinden von Wunden. Sie führten auch alle Arten von chirurgischen Eingriffen

\footnotetext{
${ }^{140}$ In den Ratsprotokollen der spätmittelalterlichen Periode befinden sich keine Angaben zur Aktivität der städtischen Behörden in der Pestzeit.

${ }^{141}$ Allgemein zum spätmittelalterlichen Medizinalwesen in Augsburg vor allem: GENSTHALER, Medizinalwesen; DIEMINGER, Bader.

${ }_{142}$ Gensthaler, Medizinalwesen, S. 46-51; Dieminger, Bader, S. 33-35, 47-53; Hoffmann, Bäder, S. 1-36; ECKER-OFFENHÄUSER, Pest, S. 28-33; HAMMOND, Origins, S. 74ff.
} 
durch, ließen zur Ader und beschäftigten sich mit dem Badewesen. Im Gegensatz zu den studierten Ärzten mussten sie sich der Behandlung innerer Krankheiten grundsätzlich enthalten. Dieses Verbot basierte auf der Vorstellung von der Zweiteilung des menschlichen Körpers in die äußere und in die innere Sphäre, die getrennt zu behandeln waren ${ }^{143}$. Dieser medizinische Grundsatz wurde aber im Spätmittelalter nicht allzu streng gehandhabt, weil die Kompetenzfragen zwischen Wundärzten und gelehrten Doktoren noch in der ersten Hälfte des 16. Jahrhunderts vom Rat geregelt wurden ${ }^{144}$. Urkundliche Belege über die ersten Bader reichen in die erste Hälfte des 13. Jahrhunderts zurück. Dagegen wurde ein Wundarzt erstmals in einer Urkunde aus dem Jahr 1320 erwähnt ${ }^{145}$. In den Augsburger Steuerbüchern notierte man Bader, Wundärzte und Barbiere bis zum Beginn des 16. Jahrhunderts nicht als getrennte Berufe, sondern fasste alle Mediziner unter dem Begriff „Bader“ zusammen ${ }^{146}$. Im 15. Jahrhundert bildeten die Bader eine bedeutende Medizinergruppe in Augsburg: In den Steuernbüchern verzeichnete man entsprechend 16 Bader für das Jahr 1434, 19 für 1448, 18 für 1462, 20 für 1475 und 22 für 1489. Diese Zahlen beziehen sich aber vermutlich nur auf solche Bader, die als Inhaber einer Badestube fungierten. Im Jahr 1475 vermerkte der Rat bei der Mitgliederzählung von städtischen Zünften nämlich insgesamt die beträchtliche Anzahl von 31 Badern, die sich zu jener Zeit in Augsburg mit Wundarznei und Badewesen beschäftigten.

Im spätmittelalterlichen Augsburg waren auch einige Ärzte ansässig, die ihre medizinische Ausbildung an den Universitäten erhalten hatten. Dank des Studiums an den medizinischen Fakultäten galten sie als die einzigen Mediziner, die über hinreichende Gelehrsamkeit verfügten, was die nicht sichtbaren Vorgänge im Mikrokosmos des menschlichen Körpers betraf. Deswegen bezog sich ihre Tätigkeit vornehmlich auf die Behandlung innerer Krankheiten. In einer Urkunde aus dem Jahr 1246 wurde zum ersten Mal ein magister Albertus Physikus erwähnt, mit dem vermutlich ein studierter Mediziner gemeint ${ }_{i s t}{ }^{147}$. Ab Mitte des 14. Jahrhunderts findet man in den Baumeisterrechnungen Einträge über meistens zwei Ärzte, die von der städtischen Kasse besoldet wurden ${ }^{148}$. Dabei ist nicht eindeutig vermerkt, ob es sich hier um studierte Ärzte oder Wundärzte handelte. Erst mit der

\footnotetext{
${ }^{143}$ Zur Zweiteilung des menschlichen Körper in der Frühen Neuzeit in Augsburg: STEIN, Franzosenkrankheit, S. 140-167; und in Bologna: PoMATA, Contracting a Cure, S. 120-139.

${ }^{144}$ Siehe S. 184-185.

${ }^{145}$ Gensthaler, Medizinalwesen, S. 46, 49.

${ }^{146}$ DIEMINGER, Bader, S. 47-53.

147 GensthaleR, Medizinalwesen, S. 40. Zur gelehrten Ärzteschaft im spätmittelalterlichen Augsburg wurde bisher keine spezielle Monographie abgefasst, wodurch nur die allgemeinen Aussagen zu dieser Frage möglich sind.

${ }^{148}$ DIEMINGER, Bader, S. 18-19.
} 
Anstellung von Doktor Herman Schedel im Jahre 1457 lässt sich zum ersten Mal ein promovierter Arzt als Stadtarzt eindeutig nachweisen. Mit der jährlichen Besoldung von 100 Gulden war er 20 Jahre lang in der Stadt tätig. Seither stellte man dauerhaft zwei Doktoren in Augsburg zu solchem Dienst ${ }^{149}$. Außer diesen zwei Stadtärzten waren in der zweiten Hälfte des 15. Jahrhunderts noch andere Doktoren in der Stadt ansässig, deren Anzahl in Augsburg zeitweise sogar bis auf sechs Mediziner anwuchs. Diese konnten ihre Heilkunst auch ohne feste Anstellung, frei praktizierend oder z.B. als Leibärzte des Augsburger Bischofs und Domkapitels ausüben.

Seit der zweiten Hälfte des 13. Jahrhunderts gab es bereits eine Apotheke in Augsburg. Apotheken beschäftigten sich ursprünglich mit Gewürzhandel und Zubereitung von Süßwaren und spezialisierten sich schrittweise im Laufe des Spätmittelalters vollständig auf die Herstellung und den Vertrieb von Arzneien. Im 13. und 14. Jahrhundert bestand nur diese eine Apotheke in Augsburg. Aber bis zur Mitte des 15. Jahrhunderts wurden noch drei weitere Apotheken eingerichtet ${ }^{150}$.

Darüber hinaus war noch eine Gruppe von unprofessionellen Laienheilern in Augsburg tätig, die mit den vom Rat anerkannten Medizinern auf dem Gesundheitsmarkt im Wettbewerb stand. Diese betrieben ihre Heilkunst ohne Kontrolle des Rates und behandelten vor allem die ärmeren Kranken. Wie die noch zu besprechenden Maßnahmen der städtischen Obrigkeit zur Eindämmung der sogenannten Pfuscherei in der ersten Hälfte des 16. Jahrhunderts zeigen werden, bildeten die Heiler ohne medizinische Ausbildung in Augsburg eine zahlenmäßig große Gruppe ${ }^{151}$.

Obwohl sich die grundlegenden Strukturen des Medizinalwesens schon im spätmittelalterlichen Augsburg herausbildeten, spielte der medizinische Pestdiskurs damals aus vielerlei Gründen nur eine untergeordnete Rolle. Die medizinische Fürsorge während der Pestzeit war zu diesem Zeitpunkt noch eine reine Privatangelegenheit und zudem nur solchen Bürgern zugänglich, die selbst einen Arzt bezahlen konnten. Aus diesem Grund waren die Armen von der ärztlichen Betreuung weitgehend ausgeschlossen oder stattdessen auf die Behandlung durch die unausgebildeten Heilkünstler angewiesen. Außerdem gab es noch keine städtische Ordnung für die Pestzeit, die alle Stadtbewohner zu vorher festgelegten Verhaltensregeln hätte veranlassen können. Die Pestbewältigung fand daher vor allem individuell statt und wurde nur in geringem Maße vom Rat getragen. Darüber hinaus ist die Verbreitung medizinischer Kenntnisse innerhalb breiterer Bevölkerungsschichten als gering

\footnotetext{
${ }^{149}$ GenSTHALER, Medizinalwesen, S. 42-46.

${ }^{150}$ Zum Augsburger Apothekenwesen vor allem: GensthALER, Medizinalwesen, S. 17-40, 52-57.

${ }^{151}$ Siehe S. 182-185.
} 
einzuschätzen. Die städtischen Doktoren oder Wundärzte als bis dato einzige Träger des medizinischen Diskurses konnten sich allein mit ihrer Pestlehre nur schwer durchsetzen. Ohne eine obrigkeitliche Pestpolitik, die zum einen auf den Grundsätzen der Medizin basierte und zum anderen durch städtische Verordnungen und deren wirksamen Umsetzung bestimmte medizinische Vorstellungen weiter in die städtische Gemeinschaft hineintrug, konnte der medizinische Pestdiskurs im spätmittelalterlichen Augsburg keine große Relevanz erlangen. 


\section{Religion und Medizin: Augsburger Pestdrucke bis zur Mitte des 16.}

\section{Jahrhunderts}

Der Ausbruch der verheerenden Pestepidemien im spätmittelalterlichen Europa rief von Anfang an ein Bedürfnis nach Sinndeutung dieses aus moderner naturwissenschaftlicher Sicht biologischen Phänomens hervor. Abgesehen von der religiösen Auslegung der Pest als einer gerechten Gottesstrafe versuchte man auch mittels der damaligen Medizinlehre das Wesen der neuen Krankheit zu deuten. Obwohl eine gründliche Erklärung des grossen sterbens die spätmittelalterlichen Mediziner vor höchste Herausforderungen stellen musste, wurden bereits 1348 die ersten medizinischen Erörterungen über Ursachen und Heilung der Pest abgefasst ${ }^{152}$. In der Zeit des Schwarzen Todes entstanden nämlich in fast allen Ländern Europas zahlreiche medizinische Pestraktate. Neben anonymen Texten, deren Autorschaft bisher ungeklärt ist, waren die Verfasser dieser medizinischen Abhandlungen gelehrte Ärzte wie z.B. Pierre de Damouzy in Reims, Johannes dela Penna in Neapel, Alfons von Cordoba oder auch von arabischen Gelehrten in Andalusien wie Ibn Hatimah aus Almeria oder Ibn al-Khatib. Einige dieser Pestschriften wurden auf obrigkeitliche Veranlassung hin von Territorialherren und Stadträten zusammengestellt, wie die Berichte über die Pest von Jacme d'Agramont im Auftrag des Stadtrates von Lérida oder von Gentile da Foligno auf eine Anfrage der Universität und des Stadtrates von Perugia. Im Jahr 1348 wurde auch das berühmte Compendium de epidemia von gelehrten Medizinern der Pariser Universität erstellt ${ }^{153}$. Den entscheidenden Anlass zur Abfassung dieses Textes gab ein Befehl König Philipps VI. von Frankreich, der durch den Tod seiner Frau Johanna auch persönlich von der Epidemie betroffen war. Bereits kurze Zeit nach seiner Abfassung ist dieser Traktat schon in vielen Abschriften und landessprachlichen Übersetzungen nachweisbar.

Das vermehrte Bemühen der Mediziner um Aufklärung und Erhellung der Hintergründe dieser folgenschweren Seuche führte folglich dazu, dass seit Mitte des 14. Jahrhunderts eine Fülle verschiedener medizinischer Schriften über diesen Gegenstand abgefasst wurde. Die intensive Auseinandersetzung der damaligen Ärzte mit dem Pestphänomen kommt deutlich bei der Betrachtung der Liste von Pestschriften zutage, die Karl Sudhoff im Archiv für Geschichte der Medizin in den Jahren 1911-1925 publiziert hat ${ }^{154}$. Während seiner

\footnotetext{
${ }^{152} \mathrm{Zu}$ den ersten medizinischen Pesttraktaten vor allem: BREHER, Memminger Stadtarzt, S. 17-37; SCHWALB, Pariser Gutachten, S. 136-157; CAMPBELL, Black Death.

${ }_{153}^{15}$ SCHWALB, Pariser Gutachten, S. 1-11.

154 SudHOFF, Pestschriften. Die Untersuchungen von Karl Sudhoff bilden nach wie vor das wichtigste bibliographische Hilfsmittel zu den medizinischen Abhandlungen über die Pest, die zwischen 1348 und 1500 verfasst wurden.
} 
langjährigen Forschungen konnte er für den Zeitraum vom Beginn des Schwarzen Todes bis um das Jahr 1500 insgesamt 281 medizinische Texte über die Pest in Europa auffinden und teilweise edieren. In diesen medizinischen Abhandlungen ergründeten die gelehrten Mediziner sowohl die Ursachen der Pest als auch mögliche vorbeugende Maßnahmen und Behandlungsmethoden.

Bei der Analyse dieser Texte lässt sich feststellen, dass alle überlieferten Pestschriften aus dem 14. und 15. Jahrhundert im Wesentlichen immer die gleichen Grundsätze in Ätiologie, Prophylaxe und Therapie enthalten ${ }^{155}$. In allen Quellenstücken weisen die medizinischen Texte um 1500 weitgehende Übereinstimmungen mit den ältesten Pestschriften aus der Mitte des 14. Jahrhunderts auf. Das andere wesentliche Merkmal des spätmittelalterlichen Pestschrifttums ist die Tatsache, dass sie vorwiegend in lateinischer Sprache verfasst wurden und in erster Linie für den Gebrauch von gelehrten Medizinern konzipiert waren. Auch aufgrund ihrer handschriftlichen Abfassung konnten sie keinesfalls ein größeres Lesepublikum erreichen. Aus diesen Gründen ist als gesichert anzunehmen, dass die handschriftlichen Pesttraktate aus dem 14. und 15 Jahrhundert vor allem unter gelehrten Medizinern zirkulierten und darüber hinaus im Spätmittelalter nicht rezipiert wurden.

Die Verbreitung medizinischen Wissens außerhalb des angestammten Gelehrtenkreises wurde erst durch die Erfindung eines neuen Mediums in der zweiten Hälfte des 15. Jahrhunderts ermöglicht: die Erfindung des Buchdrucks ${ }^{156}$. Anders als bei handschriftlichen Texten war es seither möglich, medizinisches Wissen gleichzeitig an viele Menschen weiterzugeben. Durch diese neue Art der Wissensvermittlung eröffnete sich erstmalig die Möglichkeit zur raschen Popularisierung der spätmittelalterlichen Medizinlehre. Der medizinische Diskurs fand erst seit dieser Zeit in größerem Maße Eingang in die Vorstellungswelt der Zeitgenossen des vormodernen Zeitalters. Auch die spätmittelalterliche Pestlehre konnte mit Hilfe der gedruckten Bücher ein breiteres Lesepublikum erreichen. Denn bereits seit den Siebziger Jahren des 15. Jahrhunderts wurden die ersten deutschsprachigen Pestschriften gedruckt ${ }^{157}$. Bis 1500 erschienen insgesamt etwa 70 verschiedene Ausgaben von Pestdrucken in 21 deutschen Städten, wobei der Schwerpunkt der Drucklegung derartiger Schriften eindeutig im süddeutschen Raum lag. Zu dieser Zeit wurden in Augsburg 17, in

\footnotetext{
${ }^{155}$ BREHER, Memminger Stadtarzt, S. 17-37; CAMPBELL, Black Death.

${ }^{156}$ Zur Bedeutung der medizinischen Buchdrucke in: FeuersteIN-HeRZ, Im Druck; TELLE, Pharmazie, S. 108109; SCHENDA, Der „gemeine Mann“, S. 10; KLEBS, Geschichtliche Untersuchungen, S. 85-88. Allgemein zur Entwicklung des Buchdruckwesens in Augsburg in: GIER/JANOTA (Hrsg.), Augsburger Buchdruck und Verlagswesen.

${ }^{157}$ KLEBS, Geschichtliche Untersuchungen, S. 88-100.
} 
Nürnberg 7 und in Ulm 5 Ausgaben der medizinischen Pestinkunabeln herausgegeben ${ }^{158}$. Schon aufgrund dieser Zahlen lässt sich die herausragende Stellung Augsburgs als Druckort im frühen Verbreitungsprozess der medizinischen Pestlehre gut erkennen.

\section{Religiöse Pestblätter: Schutzheilige in der Pestzeit}

Bevor sich aber die Augsburger Drucker den medizinischen Pestschriften zuwandten, hatten sie schon einige Zeit zuvor religiöse Pestblätter gedruckt. In den Sechziger Jahren des 15. Jahrhunderts, vermutlich während des Pestzuges von 1462/63, wurde in Augsburg ein Bilddruck mit einer Darstellung des strafenden Gottes angefertigt, der im Zusammenhang mit einer Pestepidemie zu deuten ist ${ }^{159}$. Am oberen Rand des Bildes thront Gottvater auf einer himmlischen Wolke, der seine Pfeile auf die sündige Menschheit abschießt (Abb. 1). Darunter kniet Jesus auf seinem Kreuz leicht erhöht zur Rechten Gottes und zeigt Gottvater, der seinen Bogen schon gespannt hat, seine Wundmale. Auf der rechten Bildseite weist die kniende Gottesmutter Maria mit einer Hand auf ihre Brust als Muttersymbol und breitet mit der anderen Hand ihren Schutzmantel über den betenden Menschen. In diesem Pestblatt tritt die im Spätmittelalter allgemeingültige Auslegung der Pest als einer Gottesstrafe deutlich hervor $^{160}$. Der Pestausbruch wird hier mit einem populären Bildmotiv des göttlichen Pfeilschützen anschaulich erklärt, das vor allem auf Texte aus dem Alten Testament zurückzuführen ist, wie z.B. in der Geschichte Hiobs, dessen Krankheit durch göttliche Pfeile hervorgerufen wurde (Hiob 6,4). Auch die große Bedeutung der heiligen Fürsprecher während der Pestzeit kommt hier deutlich zum Ausdruck. Die Menschen nehmen wegen ihrer Sünden eine gerechte himmlische Strafe hin, aber gleichzeitig rufen ihre heiligen Vermittler um Fürsprache für sie bei Gottvater an. In diesem Bild ist also folgender Gedankengang der religiösen Pestbewältigung abzulesen: Die Menschen bitten zuerst Maria als ihre heilige Mittlerin um Fürsprache bei ihrem Sohn. Danach wendet sich die Gottesmutter an diesen, damit er Gottvater um Gnade ersuchen möge, was Christus schließlich tut. Bei dieser kollektiven Fürbitte erfüllen die Brust- und Wundweisungen die Funktion der für die Menschheit geleisteten Heilstaten, wodurch eine Abwendung der göttlichen Peststrafe erfolgen kann.

\footnotetext{
${ }^{158}$ Die Bibliographie der ersten gedruckten Pestschriften in: KLEBS, Geschichtliche Untersuchungen, S. 16-84; Dazu auch: FEUERSTEIN-HERZ, Im Druck, S. 29.

${ }^{159}$ Einblattdruck, Bild des strafenden Gottes (1) [Augsburg: um 1460-1470].

${ }^{160}$ Die detaillierten Studien zu religiösen Bildern auf den Pestblättern in, EsSER, Pest, hier S. 231-235, $244-249$. Zur religiösen Wahrnehmung und Deutung der Pest im Spätmittelalter und in der Frühen Neuzeit auch: BULST, Die Pest verstehen; DERS., Heiligenverehrung.
} 


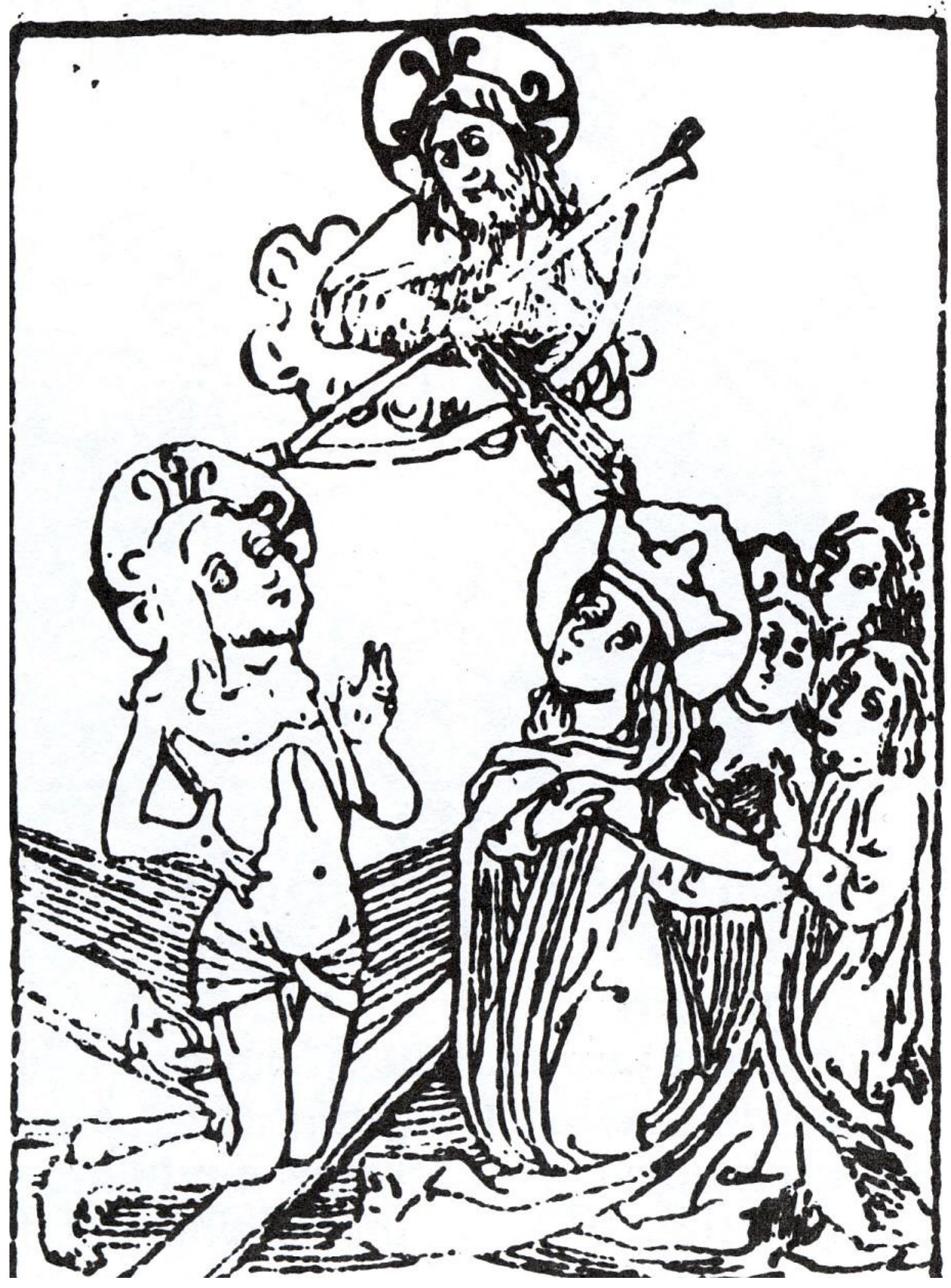

Abb. 1: Einblattdruck, Bild des strafenden Gottes (1) [Augsburg: c. 1460-1470] 
Der ähnliche Einblattdruck stammt aus den Siebziger Jahren des 15. Jahrhunderts, auf dem anstatt der Gottesmutter Maria ein Heiliger abgebildet ist (Abb. 2) ${ }^{161}$. Weil dieser Einblattdruck in Augsburg erschienen ist und vor allem an die Stadtbewohner gerichtet war, kann man die Vermutung anstellen, dass es sich hier um den Heiligen Ulrich (um 890 - 973), Bischof von Augsburg und Schutzpatron der Stadtgemeinde handelt. Darauf weist der Bischofsornat des dargestellten Heiligen, ein Fisch in seinen Händen sowie die gotische Kirchengewölbe über der gesamten Szene auf, womit der im Jahr 1474 begonnene Umbau der bestehenden Basilika St. Ulrich und Afra als ein gotischer Backsteinbau angedeutet sein könnte ${ }^{162}$.

Zwischen 1460 und 1463 erschien in Augsburg oder in Ulm auch ein Pestblatt mit der Darstellung zweier Schutzheiliger: Sebastian und Rochus ${ }^{163}$. Auch diesmal thront Gottvater im Himmel auf einer Wolke und hält ein Schwert in seinen Händen (Abb. 3). Darunter befinden sich die zwei knienden Heiligen Sebastian und Rochus in betender Körperhaltung. Zu ihren Füßen liegen zwei von der Seuche betroffene Männer, deren Körper mit Pestbeulen verunstaltet sind. Die Figur des Heiligen Sebastian ist traditionsgemäß als ein von Pfeilen durchbohrter Leib dargestellt ${ }^{164}$. Der Legende nach bekannte sich Sebastian, Hauptmann der Prätorianergarde am kaiserlichen Hof, öffentlich zum Christentum, woraufhin Kaiser Diokletian $(† 313 / 316)$ ihn zum Tode verurteilte und durch Bogenschützen erschießen ließ. In dem Glauben, er sei tot, ließ man ihn danach am Hinrichtungsort liegen. Er wurde jedoch von den Pfeilen nicht getötet. Eine fromme Witwe namens Irene hatte ihn als lebend erkannt und pflegte ihn wieder gesund. Nach seiner Genesung trat Sebastian dem Kaiser öffentlich entgegen und bekannte sich erneut zum Christentum. Diokletian befahl daraufhin, ihn mit Keulen im Circus zu erschlagen. Der Heilige Sebastian galt zuerst als ein Patron aller Kriegsleute und wurde im Laufe des Spätmittelalters aufgrund seiner Pfeilmarter zum wichtigsten Schutzheiligen gegen die Pest umgedeutet. Seine lebhafte Verehrung beruht ganz grundlegend auf der direkten gedanklichen Verknüpfung seines Martyriums mit der religiösen Vorstellung der Pest als einer durch tödliche Pestpfeile zu den Menschen geschickten göttlichen Strafe. Daher konnte er in Pestzeiten zum wirkungsvollen Fürsprecher werden, der durch die Kraft seines Martyriums mächtig genug ist, die strafenden Pfeile Gottes abzuhalten. Der Heilige Rochus ist dagegen mit einer Pestwunde am Oberschenkel dargestellt, die von einem Engel fürsorglich behandelt wird.

\footnotetext{
${ }^{161}$ Einblattdruck, Bild des strafenden Gottes (2) [Augsburg: um 1470-1480]. Dazu vgl. EsSER, Pest, S. 248-249.

162 Zum Heiligen Ulrich: RUMMEL, Ulrich von Augsburg.

${ }^{163}$ Einblattdruck, Bild der Heiligen Sebastian und Rochus, [Augsburg oder Ulm: um 1460-1463]. Zum Thema der Heiligenverehrung in der Pestzeit: BuLST, Heiligenverehrung; MÜLLER., Die Pest - Pestheillige.

${ }^{164}$ ESSER, Pest, S. 256-259; DORMEIER, Schutzpatrone, S. 60f.
} 


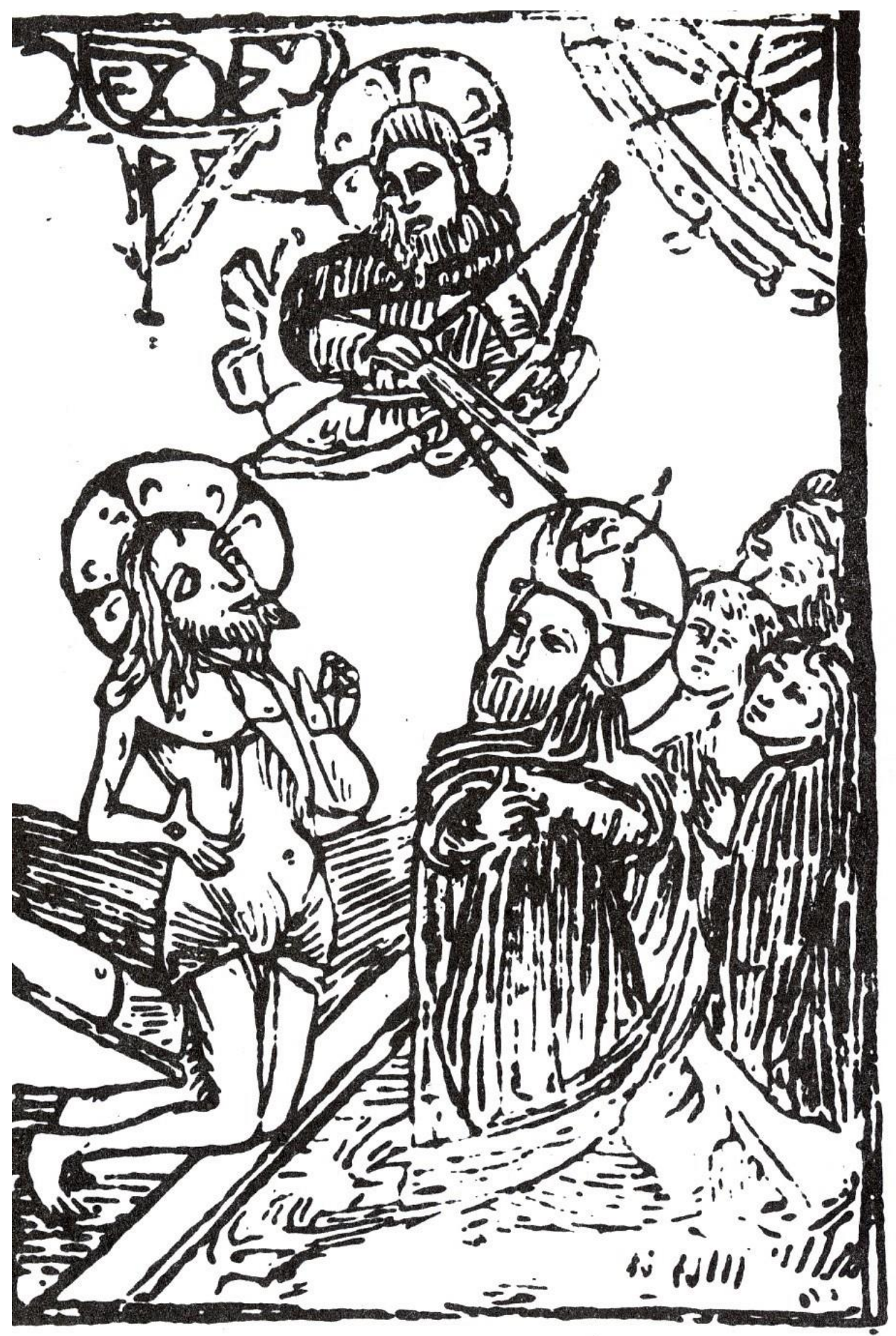

Abb. 2: Einblattdruck, Bild des strafenden Gottes (2) [Augsburg: c. 1470-1480] 


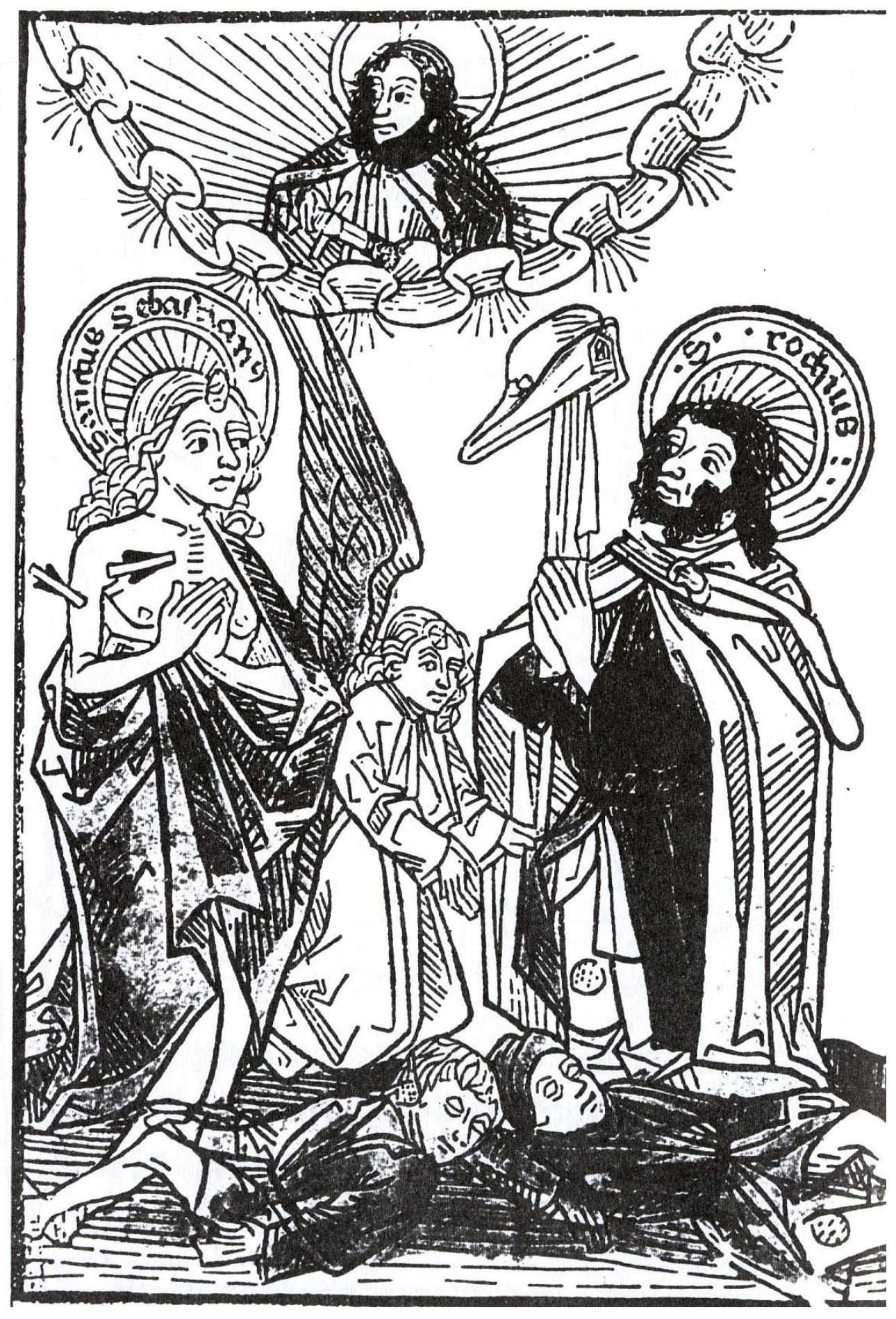

Abb. 3: Einblattdruck, Bild der Heiligen Sebastian und Rochus, [Augsburg oder Ulm: c. 1460-1463] 
Sein wichtigstes Verdienst im Kontext der religiösen Pestbewältigung erwarb er dadurch, dass er Pestkranke gepflegt haben soll und anschließend selbst an der Pest erkrankte ${ }^{165}$. Nach der mittelalterlichen Rochuslegende hatte ihm Gott zu seiner Hilfe einen Schutzengel gesandt, der seine Pestbeulen vollständig heilen konnte. Die wundersame Botschaft seiner Heilungsgeschichte ist ein Versprechen, dass jeder, der auf die Fürsprache des Heiligen hofft, auch von der Pest befreit werden könne. Diese Vorstellung prädestinierte den heiligen Rochus für die Rolle eines bevorzugten Schutzpatrons während der Pestzeit.

Ein anderes Augsburger Pestblatt, das mit dem vorherigen in der Bildkomposition weitgehend übereinstimmt, stammt aus der Zeit um $1473^{166}$. Dieses Mal wurden die bildlichen Darstellungen jedoch zusätzlich um zwei Gebete zu den Pestheiligen Sebastian und Rochus ergänzt (Abb. 4). In diesen Gebetstexten werden grundsätzlich die Verdienste der angerufenen Heiligen in den Vordergrund gestellt, aufgrund derer sie zu wirkungsvollen Fürsprechern in der Pestzeit werden können ${ }^{167}$. Außerdem enthalten sie auch eine ausdrücklich formulierte Bitte um den Schutz vor der Pest. Die Logik solcher Pestgebete besteht im Allgemeinen darin, dass man sich zunächst durch ein Anrufen der Pestheiligen die Wirksamkeit ihrer speziellen Verdienste sichert. Erst nach dem Gedenken seiner frommen Taten richtet man sich mit seiner Gnadenbitte direkt an Gott, was angesichts der Fürsprache seiner Heiligen auf eine barmherzige Verschonung von der Pest hoffen lässt.

Die zwei anderen Pestblätter, die um 1475 und um 1500 datiert sind, stellen in ihrem Bildprogramm nur das Martyrium des Heiligen Sebastians dar ${ }^{168}$. Der an den Pfahl gefesselte Sebastian wird von der daneben stehenden Schützengruppe mit Bogen- und Armbrustpfeilen beschossen (Abb. 5-6). Auch zu diesen Bildern wurden im unteren Teil des Blattes kurze Gebete an den heiligen Sebastian zum Schutz vor der Pest hinzugefügt. Das Sebastian-Motiv auf den gedruckten Pestblättern gehörte sowohl in Augsburg wie auch in anderen Städten zu den wohl populärsten religiösen Bildthemen ${ }^{169}$.

Die Gattung der religiösen Pestblätter ist hier primär als volkstümliches Gebrauchsschrifttum der privaten Frömmigkeit anzusehen, welches sich im Spätmittelalter besonderer Beliebtheit erfreute ${ }^{170}$.

\footnotetext{
${ }^{165}$ ESSER, Pest, S. 259-60; ZELLER, Rochus; SCHMITZ-EICHHOF, St. Rochus.

${ }^{166}$ Einblattdruck, Gebete zum Heiligen Rochus und Sebastian mit ihren Bilddarstellungen [Augsburg: um 1473].

${ }^{167} \mathrm{Zu}$ detaillierten Interpretationen der Gebete zu Schutzheiligen in der Pestzeit in: ESSER, Pest, S. 273-286.

${ }^{168}$ Einblattdruck, Gebet zum Heiligen Sebastian mit seiner Bilddarstellung (1) [vermutlich in Augsburg: um 1475]: Einblattdruck, Gebet zum Heiligen Sebastian mit seiner Bilddarstellung (2) [Augsburg, Hans Froschauer: um 1500].

${ }^{169}$ Vgl. die edierten Abbildungen der religiösen Pestblätter bei ESSER, Pest, S. 411-467.

${ }^{170}$ Zur Bedeutung der religiösen Pestblätter im Spätmittelalter vor allem ESSER, Pest, S. 222-227, 313-314; vgl. auch BRANDIS, Handschriften, S.179-182; KÖRNER, Einblattholzschnitt, S. 47f.
} 


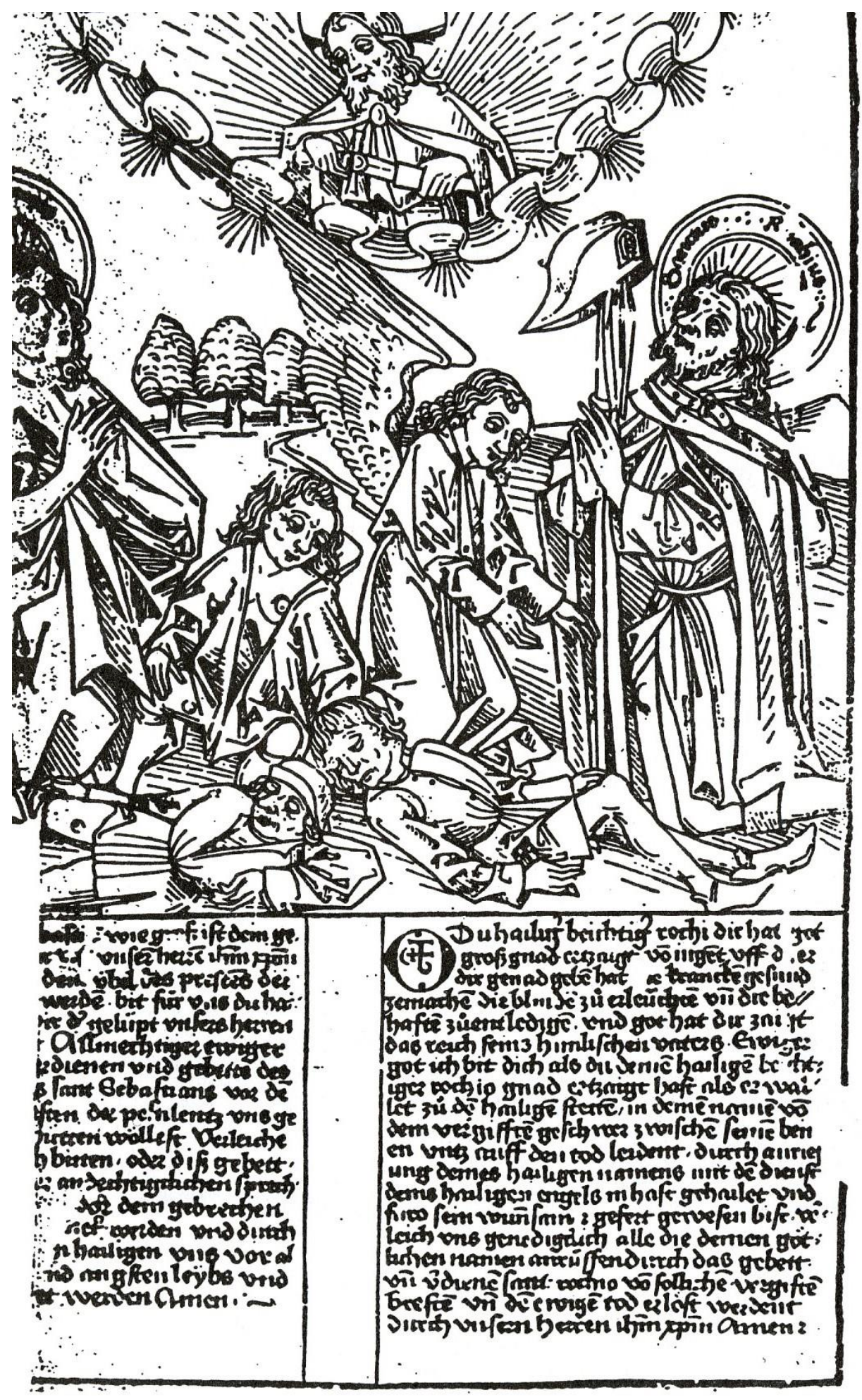

Abb. 4: Einblattdruck, Gebete zu den Heiligen Rochus und Sebastian mit ihrem Bild [Augsburg: c. 1473] 


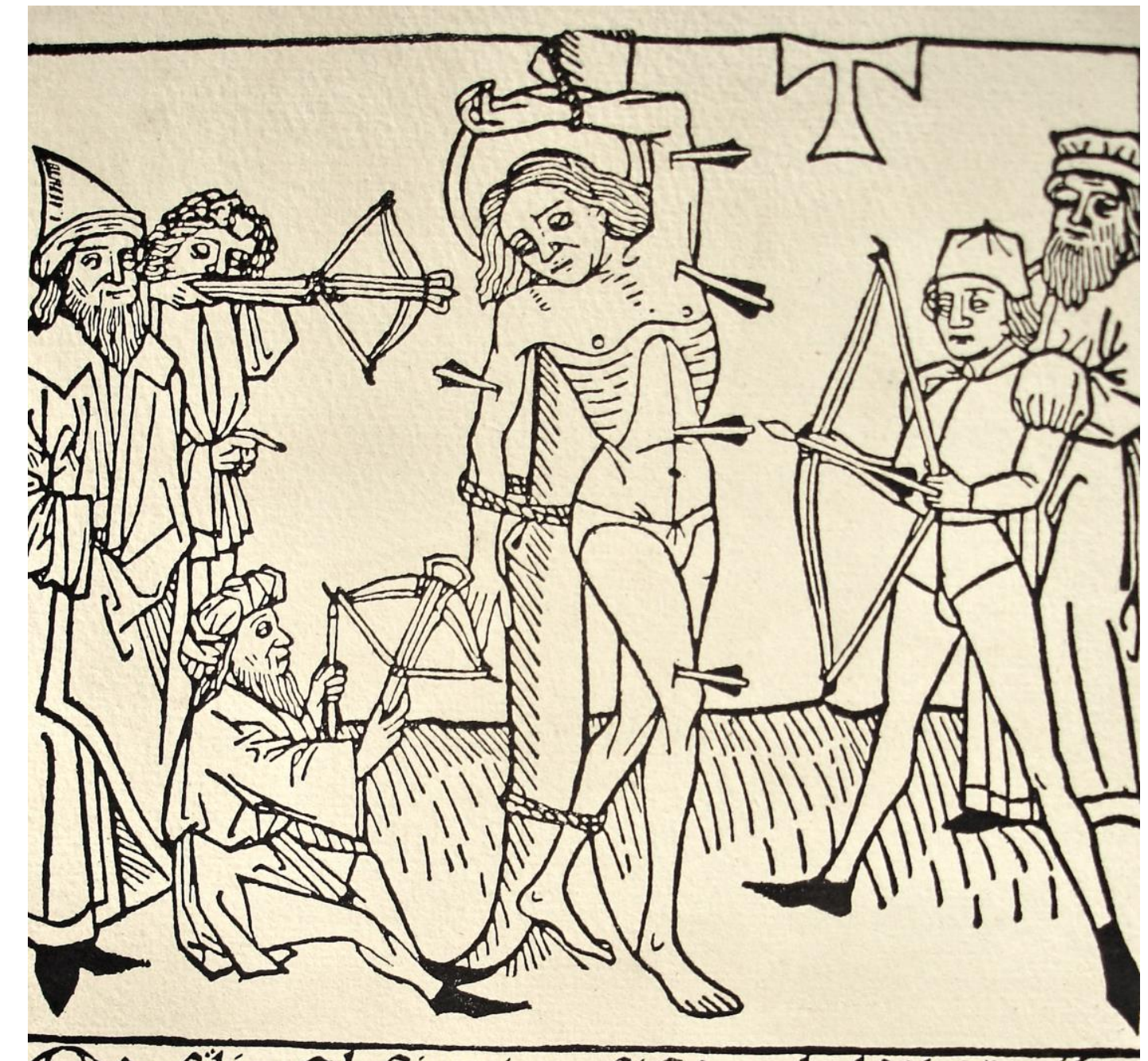

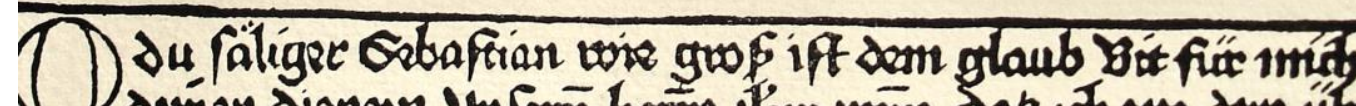

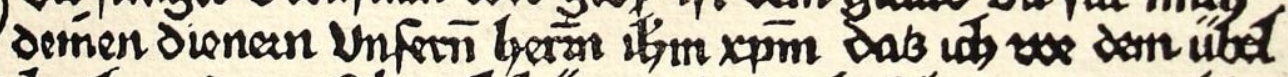

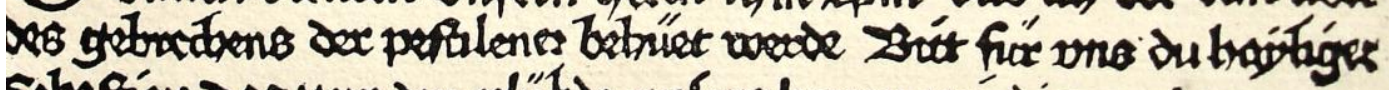

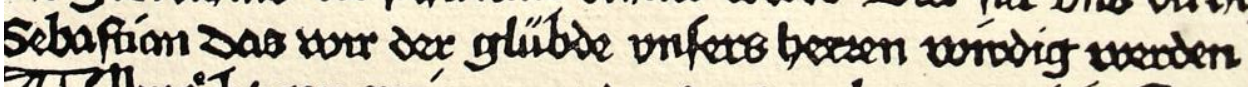

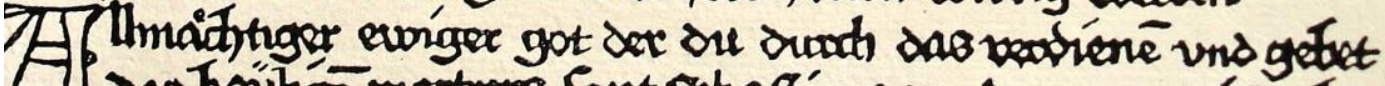

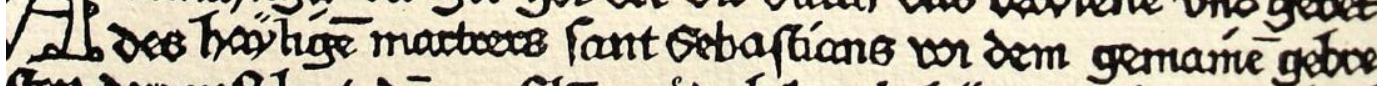

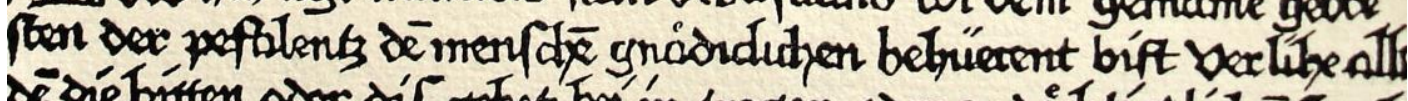

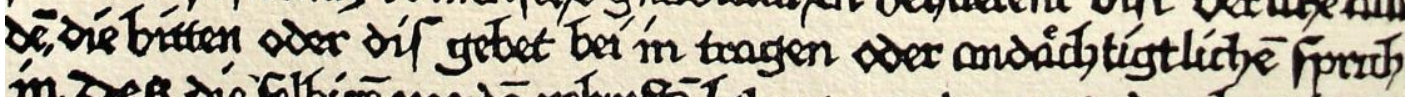

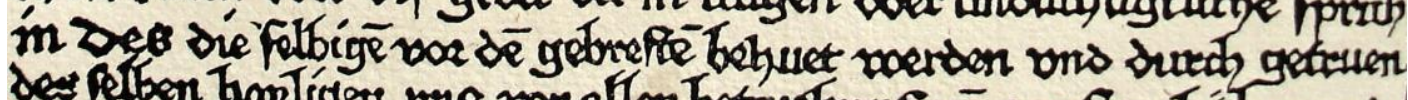

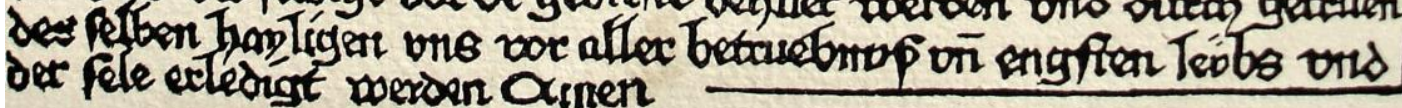

Abb. 5: Einblattdruck, Gebet zum Heiligen Sebastian mit seinem Bild (1) [vermutlich in Augsburg: um 1475] 


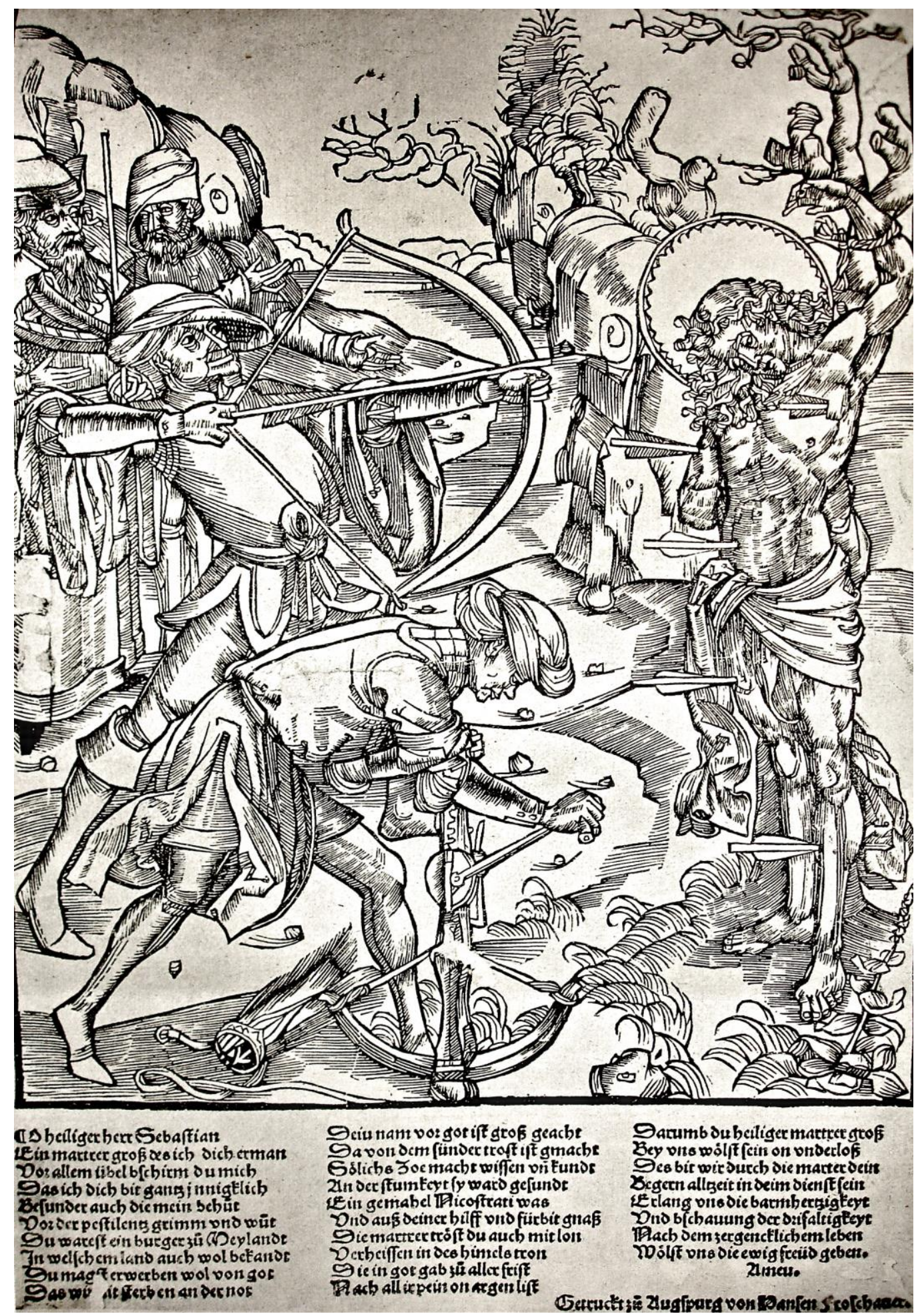

Abb. 6: Einblattdruck, Gebet zum Heiligen Sebastian mit seinem Bild (2) [Augsburg, Hans Froschauer: c. 1500] 
Schon kurz nach der Erfindung des Buchdrucks wurde dieses Medium bei der religiösen Pestbewältigung intensiv in Anspruch genommen, was der relativ große Anteil an religiösen Drucken mit Abbildungen von Pestheiligen unter allen Einblattdrucken aus der Frühdruckzeit deutlich belegt. Diese massenhaft verbreiteten Flugschriften erreichten dabei vor allem ein „Verbrauchspublikum in den mittleren und unteren Volksschichten“171.

Dies entspricht der allgemeinen Tendenz wachsender Anteilnahme aller Bevölkerungsgruppen am religiösen Leben im 14. und 15 Jahrhundert. Diese vermehrte Religiosität ist damals u.a. im Aufschwung des Wallfahrtswesens, in der gesteigerten Bedeutung der Heiligenverehrung oder in der Ausbreitung der Ablässe deutlich zu beobachten. Infolge derartiger Veränderungen der spätmittelalterlichen Frömmigkeit erhöhte sich auch der Bedarf an billigen, schnell herzustellenden Andachtsbildern beträchtlich, dem die Miniaturmaler nicht mehr in der überkommenen Weise genügen konnten. Erst mit der drucktechnischen Produktion konnte man der stetig anwachsenden Nachfrage an religiösen Bildern besser entgegenkommen. Dank der Druckerkunst steigerte sich die Bedeutung der privaten Andachtsbilder schon allein quantitativ, zumal sie jetzt auch für breitere Bevölkerungskreise zugänglich und insbesondere preislich erschwinglich waren. In diesem Kontext können die religiösen Pestdrucke vor allem als Ausdruck lebhafter Frömmigkeit innerhalb breiter Volksschichten interpretiert werden ${ }^{172}$.

Die Pestblätter erfüllten bei der individuellen Bewältigung der Pest eine wesentliche Funktion. Die in weiten Teilen der Bevölkerung beliebte Benutzung religiöser Pestdrucke basierte auf der grundlegenden Vorstellung über die wirkungsvolle Schutzmacht von Heiligen. Man sah nämlich in den bildlichen Darstellungen der Heiligen deren reale Präsenz, die Verkörperung der Heiligen selbst, mit deren Hilfe man ein Unheil wirksamer abwehren zu können glaubte. Daher lag es für die Zeitgenossen nahe, solche gedruckten Pestblätter zu erwerben, bei sich zu tragen oder in ihrer nächsten Umgebung anzubringen ${ }^{173}$.

Dafür können zwei anschauliche Beispiele aus der Augsburger Überlieferung präsentiert werden: Ambrosius Jung (geb. 1471 in Ulm - gest. 1543 in Augsburg) beschreibt in seinem Pesttraktat auch die religiösen Maßnahmen zur Versorgung der Seele in der Pestzeit ${ }^{174}$. Neben der gewöhnlichen Anrufung von Maria, St. Sebastian und St. Rochus sollte man auch religiöse Blätter zum Schutz der Kinder an deren Körper anheften, um diese zusätzlich mit dem speziellen Schutz von verschiedenen Heiligen zu versehen. Man nahm nämlich zedelin

\footnotetext{
${ }^{171}$ BRANDIS, Handschriften, S. 181.

172 EsSER, Pest, S. 225-227.

${ }^{173}$ Ebd. S. 313-314.

${ }^{174}$ JUNG, Ein außerwelt loblich tractat [Augsburg: Hans Schönsberger, 14. November 1494].
} 
und hingken die den jungen kindern an ir hels und betten sy für die kind ${ }^{175}$. Die Pestdrucke mit ihren Abbildungen von Pestheiligen und speziellen Gebettexten für die Pestzeiten scheinen diesem Zweck vorzüglich gedient zu haben. Auch in den religiösen Pestblättern finden sich einige Hinweise dazu, wie sie zu gebrauchen sind, um den speziellen Schutz von Heiligen wirksamer zu machen. In einem Gebetstext ist nämlich die Rede davon, dass man außer dem üblichen Vorlesen der Bittgebete auch selbst die Pestdrucke bei sich zu tragen pflegte: Allmächtiger ewiger got der du durch das verdienen und gebet des hayligen martrers sant Sebastians vor dem gemainen gebresten der pestilentz den menschen gnadiclichen behüerent bist verlihe allen den die bitten oder dis gebet bei im tragen oder andächtigtlichen sprechen das dieselbigen vor dem behuet werden und durch getruen des selben hayligen und vor aller betruebnus und engsten leibs und der sele erledigt werden ${ }^{176}$.

Darüber hinaus wurden die Einblattdrucke auch als heilsame Amulette an Wände, Türen oder in Wohnräumen angebracht, damit sich in diesen ihre Schutzwirkung entfalten konnte ${ }^{177}$. Auch an Altären wurden solche Drucke befestigt, die man ähnlich wie Reliquien behandelte, worin ihre enorme Bedeutung sichtbar wird ${ }^{178}$. Die Funktion der Pestblätter bestand also nicht nur darin, dem Betenden das Gebet zu formulieren oder zur Andacht anzuleiten, sondern schon allein durch ihre bloße Präsenz vor der Pest zu schützen ${ }^{179}$. Die Tatsache, dass die Zeitgenossen ein Pestblatt in ihrer persönlichen Umgebung oder gar an ihrem Leib zu befestigen pflegten, bestätigt eine stark individualisierte Frömmigkeit in der Pestzeit. Am deutlichsten zeigt dieses Phänomen ein elsässisches Pestblatt um $1500^{180}$. Im Text der darin enthaltenen Gebete steht die Variable $N$, die es dem Besitzer des Blattes ermöglichte, seinen eigenen Namen einzutragen und somit den Nutzen des Gebets speziell auf sich zu beziehen (Abb. 7). Die heilige Schutzwirkung soll sich dabei sowohl auf den Besitzer als auch auf die menschen des selben huß erstrecken.

\footnotetext{
175 Ebd. S. A Vv.

${ }^{176}$ Einblattdruck, Gebet zum Heiligen Sebastian mit seiner Bilddarstellung (1) [vermutlich in Augsburg: um 1475]; Einblattdruck, Gebete zum Heiligen Rochus und Sebastian mit ihren Bilddarstellungen [Augsburg: um 1473].

${ }^{177}$ NOCKEMANN, Einblattholzschnitt, S. 27f.

${ }^{178}$ SCHREIBER, Handbuch, Bd. 1, S. 5, Nr. 8a.

${ }^{179}$ ESSER, Pest, S. 314.

${ }^{180}$ SCHREIBER, Handbuch, Nr. 931.
} 


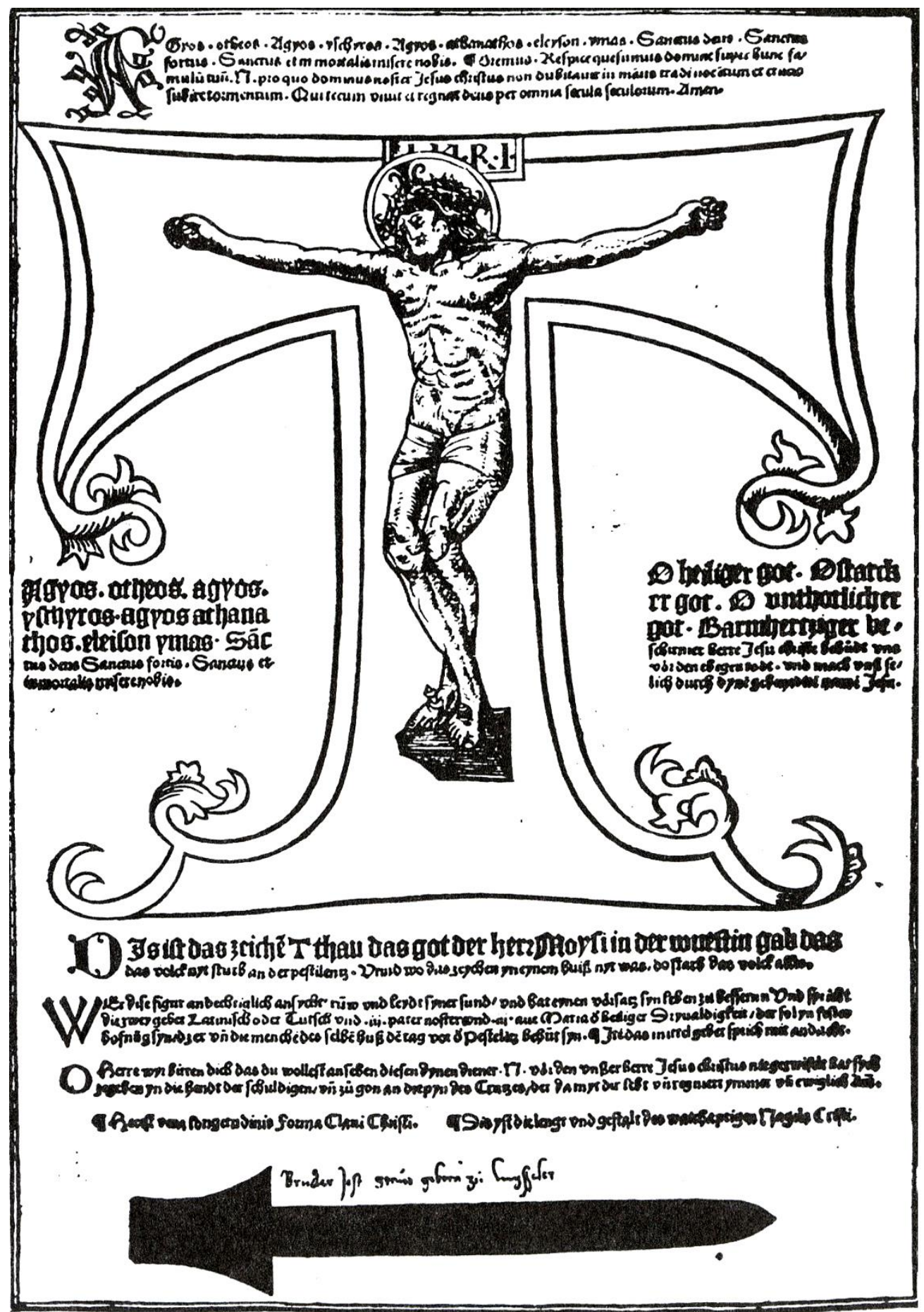

Abb. 7: Einblattdruck für die Pestzeit, [Elsass, um 1500] 


\section{Inkunabeln zur medizinischen Pestlehre}

In den Siebziger Jahren des 15. Jahrhunderts erschienen dagegen in Augsburg die ersten deutschsprachigen Inkunabeln, welche die medizinische Pestlehre beinhalteten. Als das erste medizinische Druckwerk gilt die Ordnung der Gesundheit, welche am 23. April 1472 von Johannes Bämler in Augsburg gedruckt wurde ${ }^{181}$. Das Buch wurde anhand einer handschriftlichen Vorlage zum Druck zusammengestellt, die ursprünglich um $1400 \mathrm{im}$ Umkreis des Rottenburger Hofes verfasst wurde. Abgesehen von dem heute nur noch fragmentarisch erhaltenen „Aderlasskalender“, der 1457 in der Offizin Gutenbergs herausgegeben wurde ${ }^{182}$, ist die Ordnung der Gesundheit der erste deutschsprachige Medizindruck überhaupt. In diesem medizinischen Werk, welches zu der vor allem im Spätmittelalter weit verbreiteten Literaturgattung des Regimen sanitatis gehört ${ }^{183}$, werden generell die allgemeinen Regeln der Gesunderhaltung, Prophylaxe sowie Therapie für den Laien vermittelt. In seinem dritten Teil „Von der pestilentz“ wird eine kurz gefasste Pestlehre besprochen, die zum ersten Mal mit Hilfe dieses neuen Mediums einem breiten Lesepublikum angeboten wurde. Das Werk erfreute sich in der folgenden Zeit so großer Beliebtheit, dass bis 1523 insgesamt 13 Ausgaben in Augsburg, Nürnberg, Ulm und Freiburg erschienen ${ }^{184}$. Dieses Regimen sanitatis gehörte damit zu den meist aufgelegten Büchern in den Augsburger Druckereien $^{185}$.

Auf die Zeit um 1472 sind auch zwei Einblattdrucke aus der Druckerei von Günther Zainer datiert, die in gereimter Kurzfassung die Grundsätze der medizinischen Pestlehre vermitteln ${ }^{186}$. Die zur Prophylaxe gegen die Pest erteilten Anweisungen werden aufgrund der Lehre von Meister Hans Tornamita und auch annder hübscher meyster vil erstellt ${ }^{187}$. Dieses populärmedizinische Gedicht ist vor allem an den gemeinen man gerichtet, der aus diesem die

\footnotetext{
181 HagenMeyer, 'Ordnung der Gesundheit'; SudHOFF, Inkunabeln, S. 8-13; KLEBS, Geschichtliche Untersuchungen, S, 40-42.

${ }^{182}$ SUDHOFF, Laßtafelkunst, S. $222 \mathrm{f}$.

${ }^{183}$ Vgl. SCHMITT, Regimina.

184 Nach Christa Hagenmeyer ('Ordnung der Gesundheit', S. 45-50) sind die folgenden Ausgaben von der Ordnung der Gesundheit zu ermitteln: 23. April 1472, 13. November 1475, 4. März 1476, 27. Oktober 1477, 2. September 1482 Augsburg, Johannes Bämler, um 1474 Nürnberg, Friedrich Creussner, 1. September 1481, 9. September 1482 Augsburg, Hanns Schönsberger, 5. Oktober 1482, 19. Oktober 1482 Ulm, Konrad Dunkmut, 1490 Augsburg, Anthonio Sorg, 1. April 1495 Augsburg, Hans Froschauer, 1523 Freiburg, Johannes Wörlin; In der von Jörg Künast zusammengestellten Liste sind 8 Auflagen von Regimen sanitatis (1472-1495) in Augsburg und dann 6 auswärtige Nachdrucke (bis 1555) aufgezählt: KÜNAST, Buchdruck, S. 223.

${ }^{185}$ Vgl. Tabelle: Deutsche Augsburger Erstausgaben und ihre auswärtigen Nachdrucke bis 1555 in: KÜNAST, Buchdruck, S. 223.

${ }^{186}$ TORMANITA, Vil menschen weren der pestilentz frey (1-2) [Einbl. Augsburg: Günther Zainer um 1472]; Dazu: SudHofF, Inkunabeln, S. 191; KLEBS, Geschichtliche Untersuchungen, S, 43-44. Die spätere Ausgabe desselben Einblattdruckes: ARDEA, Ain gut und vast nutzliches Regiment [Einbl. Augsburg: 1536].

${ }^{187}$ In der Druckausgabe aus dem Jahre 1536 wird dagegen Meister Hans Ardea als der Autor der erteilten Anweisungen genannt.
} 
praktischen Verhaltensregeln und Arzneien zur Pestprophylaxe entnehmen konnte. Im oberen Teil des Blattes wurde auch ein Holzschnitt mit dem Martyrium des Heiligen Sebastian beigefügt (Abb. 8), das in der zweiten Version dieses Einblattdruckes (Abb. 9) um eine zweite Darstellung von zehn nackten nur mit einem Lendenschurz bekleideten Heiligen, die in wildem Gewirr auf Dornen gespießt sind, ergänzt wurde.

Im Jahr 1473 wurde von Johannes Zainer in Ulm die erste deutschsprachige Pestschrift als eigenständiges Werk herausgegeben, die Heinrich Steinhöwel, der Ulmer Stadtarzt (um 14201482) zusammengestellt hatte ${ }^{188}$. Der Pestdruck von Steinhöwel mit Gesundheitsvorschriften zur Prophylaxe und Behandlung der Pest, dadurch sich der gemein man vast wol behelfen mag, war trotz der noch wenig strukturierten Textfassung auch ein verlegerischer Erfolg und erfuhr bis 1500 noch weitere fünf Auflagen ${ }^{189}$.

Die Anfänge der gedruckten Pestschriften auf dem deutschsprachigen Gebiet sind eng mit der Tätigkeit der genannten Augsburger Drucker Johannes Bämler und Günther Zainer und des Ulmer Druckers Johannes Zainer verbunden ${ }^{190}$. Die beiden letzteren waren trotz fehlender eindeutiger Quellenbelege vermutlich miteinander verwandt, worauf sowohl ihr gemeinsamer Herkunftsort Reutlingen als auch ihre typographische Lehrzeit in Straßburg verweisen. Beide Zainers zogen 1468 um, Günther nach Augsburg und Johannes nach Ulm, wo sie jeweils ihre Tätigkeit als Drucker erfolgreich aufnahmen. Dass die Herausgabe medizinischer Werke gerade in der ersten Entwicklungsphase in Augsburg und Ulm ihren Ursprung hatte, sah Arnold Klebs vor allem in der Tatsache begründet, dass die Druckereien in Mainz, Straßburg und Köln im Gegensatz zu diesen zwei süddeutschen Städten in erster Linie kirchliche Aufträge realisieren konnten ${ }^{191}$. Die Drucker in Augsburg und Ulm richteten sich dagegen schon früher mit ihren Büchern an neue Kundschaft und publizierten daher auch Druckwerke zu weltlichen Themen. Andererseits konnten sie für derartige Publikationen in der politisch erstarkten Bürgerschaft der süddeutschen Städte ein großes und an weltlichen Angelegenheiten interessiertes Lesepublikum mühelos gewinnen. Die Pestwellen in der zweiten Hälfte des 15. Jahrhunderts lösten dabei zusätzlich einen direkten Anstoß zur Drucklegung medizinischer Pestschriften aus. Aus diesen Gründen konnten die süddeutschen Städte, zuerst Augsburg und Ulm, in der Folge auch Nürnberg zum Vorreiter auf dem Gebiet der Herstellung medizinischer Pestdrucke werden.

\footnotetext{
${ }^{188}$ STEINHÖWEL, Büchlin der ordnung [Ulm: Johannes Zainer von Reutlingen, 11. Januar 1473].

189 KLEBS, Geschichtliche Untersuchungen, S, 16-84: 1474 Eßlingen, Konrad Fyner; 1482 Ulm, Johannes Zainer; 1482 Nürnberg; 1482 Ulm, Konrad Dinkmut; um 1500 Magdeburg, Moritz Brandis. Zu diesem gedruckten Werk vor allem: SUDHOFF, Steinhöwel; auch FEUERSTEIN, Im Druck, S. 30-31.

${ }^{190}$ KLEBS, Geschichtliche Untersuchungen, S. 88-100; SUDHOFF, Steinhöwel, S. 174.

${ }^{191}$ KLEBS, Geschichtliche Untersuchungen, S. 89-90.
} 


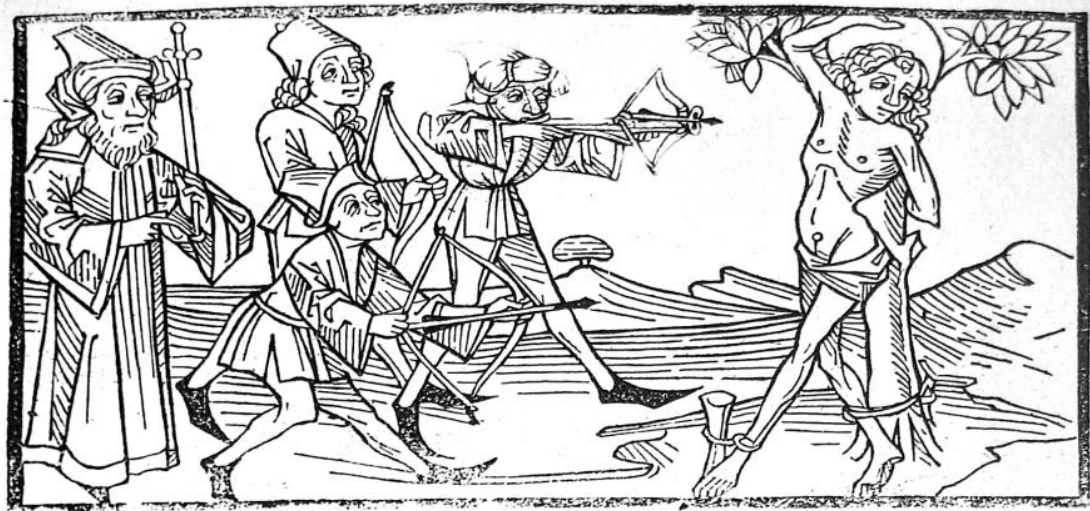

Vil menichen roeven so peitelents firep Webten [p oar fár ein rechte ertznep Darumb To hổ 20as ich oir fa gen zoil W añ alfo fterben ift gar sın hurt3 żl Deserften balt ben rat ben ich mepn Wañ 8 ouncket mich) ficher niek îngn Das man in oifer fach eeriftlich fol Ontuffen got oas bilffet ficlixe 2001 Sant Tebaltuans auch nit rergth Wañ feın belffen ift auch gar gevo:6

Das mapnen all mapiter 230 eif

Die to remo aufte febul zu paret

Datnacb bab auch ecin fllbs asbe Es fep frù fpat cber zi̊ ber naché

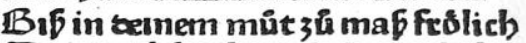
Das builch ich oir befuncerlich Du folt auch nit zů vil fróaen ban Wañ oz heré zouro zú falt auf getban Tu folt orch haiten in follteber mafien Vmo folt alle monat cinelt aberlaffen Rach ber zoeten gelecten artzet lete Von bitzigen oungen ovo oich here Roch vil mer fag ich ore oa mite Rpem auch all worbxn pullulas vite Subme Deet neúne oie zü nacbe Tchlıck Esoar Tp feino oircpn güt glick iDas lenet oich) Rafis oer mepiter gros Dem soch hepn art $3 e t$ ift genor

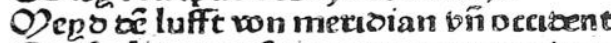
npfach in won feptentrion vilo ozient, Es ift gut vin bzingt ciel) aub lozgen

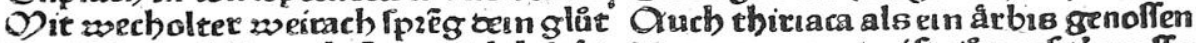
Vos bófem nebligem luft ou oich belyute Vnber cem ssepn ift giưt aú O) it furnbom wecholté mach bei ferse Es fterchet oas heres wrmaffen baft Das ift ${ }^{3}$ zet in oepnem baub gebeme Giffe mag nit Tein bep in etn gaft

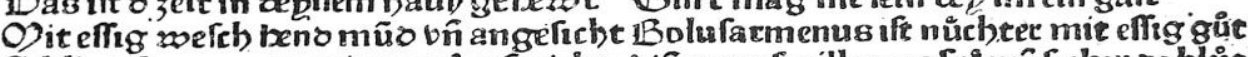

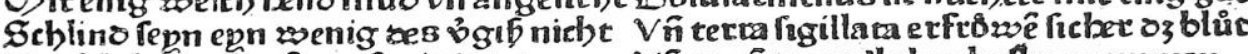
Du folt bunger vî ourft nit letoen $\checkmark$ baige fulle folt ou auch fere mepaen $\checkmark$ no ubzige grobe koft folt ou taînen

$V_{\text {or }}$ bil trincken foltu oich auch maffen Gebzaten fleifeb ift beffer oañ gefouten Das febroeingn fep oir gant3 mebotten iDu folt mifeben ven farcken zocpn Oas Techbt tepl fol all zetr zoaffer fepn Du folt nit mex feblaffen benn roachen Whate ouch ooz tê in 8 pfannê gebachen IIt es an oir nit etn gessonbett alt. ID fole auch alle tag etroas begennen Vĩi 2oañ ou pullulas haft genommen So foltu'nicbe zủ tbirtaca kommen Suncer oil mepo bif an oen anźn tas Vno neefitano z23 ich oir oa fag Roch en mapiter oux epncn wat gett

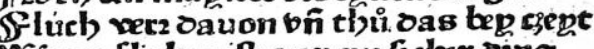
Wann flieben ift $g$ ar can ficher oing Vno balten toch etlich bas gar gering Sivich oue fiechen ono auch oie frat Gepnen rock frin gevoano oñ woz ex bat L pnfen mit gâtem effig 2001 gefotten Emfig fepn mit fró200 in oem haus

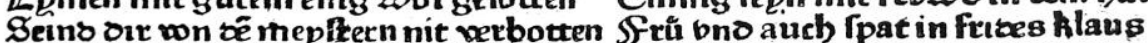
Q ber zoiltu oich Elbs nit i febaoê geben $E_{s}$ ift au/ 3 ber maffen vaft güt Sobút oich auch wo vnheuffem teben Wer oar pnnen ift mit frôlichem muit

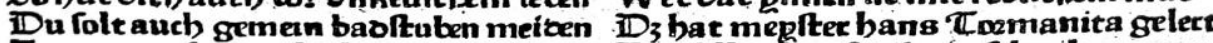

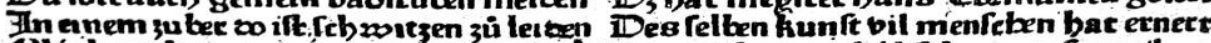

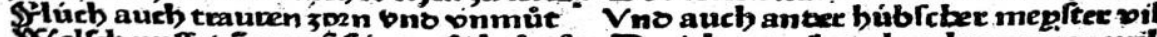

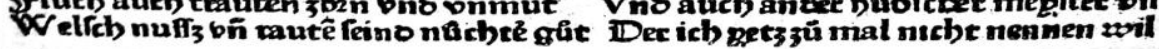

Abb. 8: ToRmANiTA, Vil menschen weren der pestilentz frey (1) [Einbl. Augsburg: Günther Zainer um 1472] 


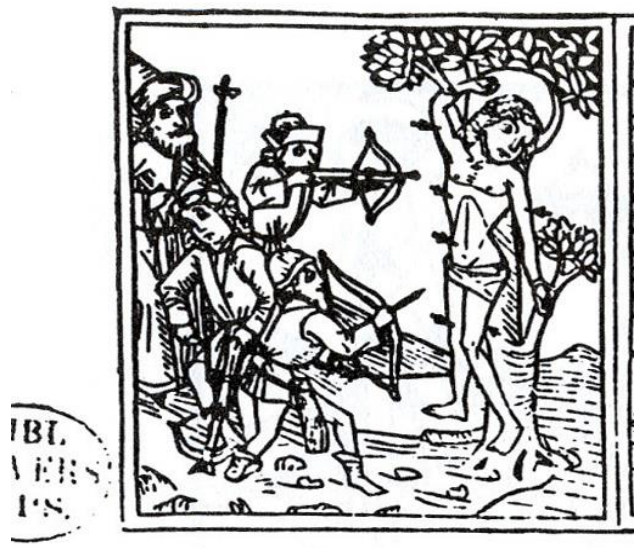

Vil menfben weren orr paftelene; frep rociten fp oar fur cin rechre extzncp

Datumb fo bóre was ich our fagen woil

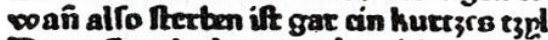
Des exiten balt oen ration icb mcin Wann cer ouncket mich fidber nit kilein Das man in oifer facb exnnfelich fol anxuffen got oas bilfer ficber 200 Sant \$ebaftians aucb nit vergi wann fcin belffen ift aucb gar geroif Das mepnen all mepiter woce dic oa feino auff ace Tdoúl zú parep̣ Dat nacb bab aucb ccin felbs acbt es fę frú fpat ooer zŭ der nacbt (1) epo oen luffe oon merioian on̂ oriocen enp facb in von feptentrion ono ocicne Dit roacboltern weizaucb Iprêg oci glủ - 200 bò fem nebligem lufr ou oudb babút Dit feuenbaum wacbolter mach xí feu Das ifr wer zeit in cainem haub geberor

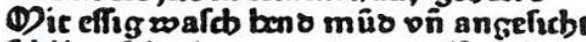
feblino fein cin ro enig oes vergif uncht Ou fole bunger ono ourfe nie lezecn ubrige fullle foleu auch fere mep ocn Vno ubrige grote kúfe foltu taffen - or vil trincken foltu oich aucb maifen Bebraten fleifeb If́ beffec oann gefoten oas febrocinen fep our ganç verboren Ou fole mifeben aen frarchicn wein òa fechfe teil fol alc;epe 20 affec fein Du fole nit mer feblafen aenn roacben bût oıcb vor ổ un zer pfannen gebacben Ifr es an oix nit cin geroonbcit alc fo fleido cen fchlaf im tag mit gewale Lprifen mit gứem effig wol geforen féno dir von cen mepfiretn nir verboren Arbex wiltu oicb felbs nte in febatcm gety To bưt oicb auch 003 vnkeifebem leben Du fole audb gamen baofruben mciacn

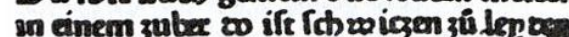
Yleucb aucb trauren zon ono vnmú

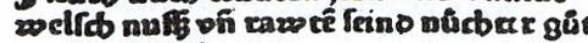

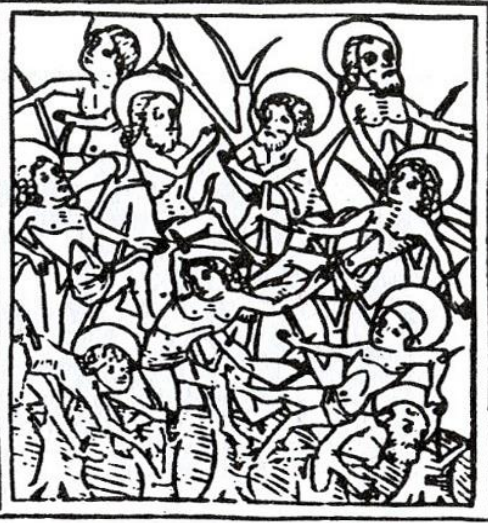

Biß̧ in rcinen mút zû mas frolicb sas beuilcb ich oir befun berlich Du folt aucb nit zư vil fereoven ban mann oas berç wuro zŭ falt auff getban Đu folt oicb hulten in fölcber mağen ono folt allic monct cinef́t acerlafen Racb wer weifen gelerten arget lere von biezigen oungen ou cicb kere Roch vil mex fag icb ore oa mite npm aucb alle roocben pillulas vite Subene osee nerone oic zư uacbe foblich zovar fie fepn our cin gúe geluch Das lext oicb Rafis act menfter gros cem wob kepn arget ift geno 3

Klle rag fablick ein pillen zú mogen es If́ gû́t ono pringe oidb auß 5 loggen Qucb tbiriaca als ein ắcbes genofîn vnoer com roan ift gàt aub uer mofien Es frecket oas beeg onmafen vafe giffe mag nicbe fepn bep im cin gafe Bolufianmenus if́t núchter mit effig gût

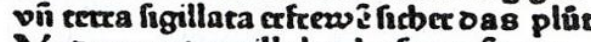
Vno wenn ou pillulas baft genúmen fo foltu niche zů tbiriaca kummen Suncer oif mepo bif an ecn anceren eag vno océteano roas icb oir oa fag Rocb cin menfret our einen rat geic flcucb vere oauon vno thú oas trp 3 ept W ann flieben ift gax cin ficber oing ono balcen cudb et! ucl, oas gar gering Gleucb oic fiecben ono aucb die frac fanen rock frin gewano ono poas extat Du folt aucb alle tag etwas byinnen zú thuin mit lcib ono aucb mit funnen

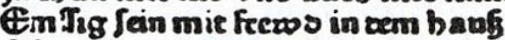
frû ono aucb fpat in friors hlauß Esift aús vex maken vafr giote roce oar pnne ife mis frolicbem mule Das bat meifrer hang Toenamien gelect Ocs felten kunit vil menfeben bat cencte Vno aucb annocr bubfeber mepiree vil ocr icb pac3 3 ămal viche nennen wo th

Abb. 9: TORMANITA, Vil menschen weren der pestilentz frey (2) [Einbl. Augsburg: Günther Zainer um 1472] 
Bis 1500 erschienen in Augsburg weitere Pestinkunabeln in verschiedenen Druckformen, die ein wachsendes Interesse für derartige medizinische Schriften deutlich bezeugen. Im Jahr 1483 wurde vermutlich von Johannes Bämler der Spruch von der Pestilentz des Nürnberger Wundarztes und Druckers Hans Folz (1435-1513) gedruckt, der ursprünglich ein Jahr früher in Nürnberg im Druck erschienen war ${ }^{192}$. Dieses gereimte Werk gibt in unterhaltsamer Form medizinische Erklärungen und diätetische Empfehlungen auff das aller kürtzest begriffen wieder, die vor allem zum Gebrauch für den gemeinen man zusammengestellt wurden. Dieser kurzweilige Gebrauchstext für die Pestzeit konnte der Absicht des Autors entsprechend auch auswendig gelernt werden.

Mit ähnlicher Intention stellte 1429 auch Heinrich Louffenberg, Priester in Freiburg i. Breisgau, sein Regimen sanitatis zusammen ${ }^{193}$. Darin werden in einem Kapitel die Grundsätze der Pestlehre besprochen. Auch dieser in Versen gedichtete Text war sicher für die Belehrung des Laienpublikums geplant. Wohl wegen der großen Beliebtheit des Druckes Ordnung der Gesundheit wurde 1491 auch das Werk von Louffenberg in Augsburg, vermutlich in der Druckerei von Erhard Ratdolt, gedruckt. Im Jahr 1490 wurde ebenfalls ein Regimen sanitatis nach der Lehre Ortolfs von Baierland veröffentlicht ${ }^{194}$. Das Werk Baierlands, welcher um 1300 als Arzt in Würzburg lebte, wurde für die Drucklegung 1490 um ein kurzes Kapitel über die Pest ergänzt ${ }^{195}$. Auch zwei Einblattdrucke aus der Zeit um 1483 sowie 1495 enthalten in kurzer Fassung allgemeine Hinweise zur Pestprophylaxe und -behandlung, die insbesondere für nicht gelehrte Leser geeignet sind ${ }^{196}$.

Am Ende des 15. Jahrhunderts wurden auch zwei Pestschriften des Augsburger Doktors Ambrosius Jung gedruckt, der um diese Zeit als Leibarzt des Augsburger Domkapitels tätig war ${ }^{197}$. Bei seinen medizinischen Werken handelt es sich um zwei Versionen einer Pestschrift, die in deutscher ${ }^{198}$ und lateinischer ${ }^{199}$ Sprache 1494 kurz nacheinander aufgelegt wurden. Durch diese parallele Veröffentlichung konnte der Autor sein Werk gleichzeitig an das Fach- wie auch an das Laienpublikum richten. Seine Pestwerke entstanden im Auftrag des Domkapitels, was auf dem Titelblatt der beiden Ausgaben in einer

\footnotetext{
${ }^{192}$ FolZ, Spruch von der Pestilentz, S. 1-18; KLEBS, Geschichtliche Untersuchungen, S. 29-30. Die spätere Ausgabe wurde um 1530 in Augsburg bei Philipp Ulhart gedruckt.

${ }_{193}$ JENTSCH, Regimen sanitatis; SUDHOFF, Inkunabeln, S. 17-20.

194 ORTOLF VON BAIERLAND, Versechung leib sele ere vnd gut [Augsburg: Hans Schobser 1490].

${ }^{195}$ Vgl. dazu: RiHA, Funktionswandel, S. 62-69.

196 Einblattdruck, Wie sich der mensch halten sol wider die pestilentz [Augsburg: Hermann Kästlin um 1483; Hans Schauer um 1495]; KLEBS, Geschichtliche Untersuchungen, S. 45; SuDHOFF, Pestblatt.

${ }^{197}$ Zum Leben von Ambrosius Jung: FleISCHMANN, Die Ärztefamilie Jung; MARTZ, Gesundheitswesen, S. 1920; SUDHOFF, Inkunabeln, S. 179-182.

198 JUNG, Ein außerwelt loblich tractat [Augsburg: Hans Schönsberger, 14. November 1494].

${ }^{199}$ DERS., Tractatulus perutilis de pestilentia [Augsburg: Hans Schönsberger, 20. November 1494].
} 
Bilddarstellung symbolisch angedeutet wurde: Der lorbeerkranzgeschmückte Autor überreicht kniefällig drei Domherren sein Buch (Abb. 10). Auch die gelehrte Form dieses Traktates mit umfangreichen akademischen Ausführungen weist auf das Domkapitel und andere gebildete Leser als das gewünschte Zielpublikum hin. Sollte jemand aber noch mehr Interesse für weitere Erörterungen haben, solle der Leser nach Absicht des Autors direkt in der lateinischen Ausgabe nachschlagen, in der auch einige Rezepte vermerkt sind, die der Verfasser nicht übersetzen konnte oder wollte. Auch ein um 1480 in Augsburg gedruckter lateinischer Pesttraktat von Johannes Jacobi, einem gelehrten Mediziner und Kanzler der Universität von Montpellier im 14. Jahrhundert, war ausschließlich für ein ausgebildetes Fachpublikum zusammengestellt worden ${ }^{200}$.

Auch Ulrich Ellenbog (gest. 1499 in Memmingen), Memminger Stadtarzt und gleichzeitig Leibarzt Erzherzog Sigismunds des Einfältigen von Tirol, der in den Jahren 1472-1478 zudem als ein Leibarzt des Augsburger Domkapitels angestellt war, betätigte sich als Autor von Pestschriften ${ }^{201}$. Schon 1464 verfasste er eine lateinische Abhandlung über die Pest, die nur in Form einer Handschrift überliefert ist. Anschließend stellte Ellenbog auf Veranlassung des Memminger Rates 1482 eine deutschsprachige Pestschrift zusammen, deren Druck aber erst durch eine Neuauflage von 1494 bekannt ist ${ }^{202}$. Sein zweiter uns bekannter Pestdruck stammt aus demselben Jahr und ist im Vergleich zum ersten Buch in deutlich umfangreicher Form von ihm erarbeitet worden ${ }^{203}$. In diesen beiden medizinischen Werken ist der Schwerpunkt erkennbar auf die praktischen Vorbeugungs- und Behandlungsmethoden gesetzt, weshalb diese Art und Weise der medizinischen Unterweisungen insbesondere für ein breites Lesepublikum geeignet waren.

\footnotetext{
${ }^{200}$ JACOBI, Tractatus de pestilentia [Augsburg: Johann Keller um 1480]. Zum Leben und Werk von Johannes Jacobi in: KLEBS, Geschichtliche Untersuchungen, S. 145-149.

${ }^{201}$ BREHER, Ulrich Ellenbog, S. 5-16.

${ }^{202}$ ELLENBOG, Ordnung [Memmingen: Albrecht Kunnen 1494].

${ }^{203}$ DERS., Ain wunderbäre instruction [Memmingen: Albrecht Kunnen 1494]; BREHER, Ulrich Ellenbog, S. 99103.
} 


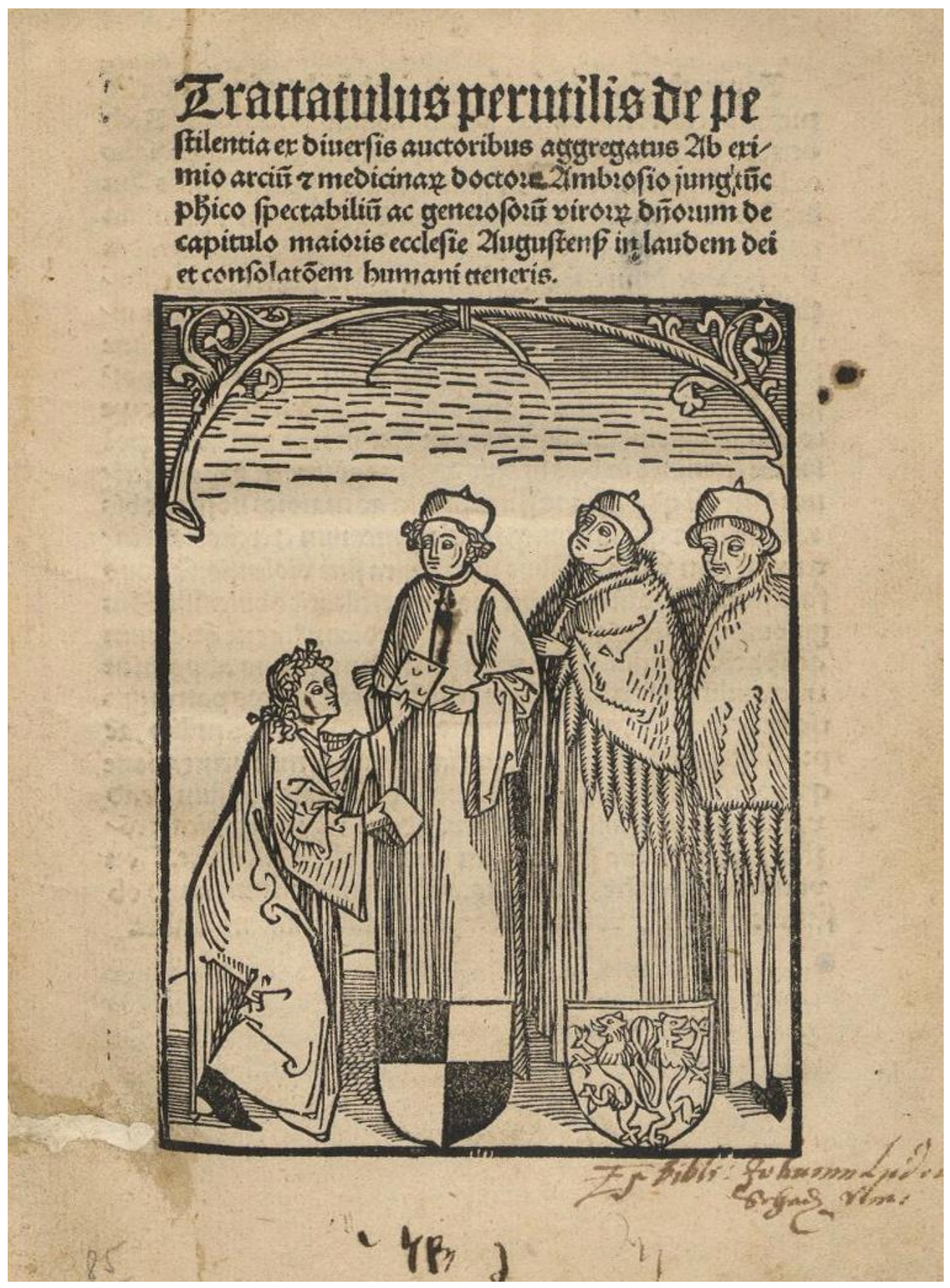

Abb. 10: Titelblatt in: Jung AmBRosius, Tractatulus perutilis de pestilentia [Augsburg: Hans Schönsberger, 20. November 1494] 


\section{Pestschriften der Augsburger Doktoren}

Bis zur Mitte des 16. Jahrhunderts erschienen in Augsburg weitere Pestschriften, die von Augsburger Doktoren verfasst worden waren. Als im Jahre 1521 die Pest ausgebrochen war, veröffentlichte Doktor Ambrosius Jung als Augsburger Stadtarzt seine nächste Pestschrift ${ }^{204}$. Im Gegensatz zu seinen zwei Pesttraktaten von 1494 wurde dieses Buch unter Verzicht auf zuviel Gelehrsamkeit abgefasst, weil die klar geäußerte Absicht des Autors darin bestand, dem gemainen armen man zum trost und hilff/ die dann nit alle zeit Doctores haben mag zu schreiben $^{205}$. Wohl wegen der praktischen Ausrichtung seiner Pestlehre, die vor allem leicht anwendbare Prophylaxe und Behandlungen gegen die Pest vermittelt, erlangte seine Pestschrift große Beliebtheit in Augsburg. Bis in die sechziger Jahre des 16. Jahrhunderts hinein wurde dieser Pesttraktat mit einigen Änderungen noch dreimal in Augsburg und je einmal in Innsbruck und Straßburg aufgelegt ${ }^{206}$.

Im Pestjahr 1521 brachte noch ein anderer Augsburger Doktor namens Johann Klainmüller (genannt Wohlopter) sein Buch über die Pest zum Druck ${ }^{207}$. Trotz einiger gelehrter Ausführungen im Kapitel über die himmlischen Ursachen der Pest kann man auch diese Pestschrift eher als ein populäres Werk für den gemeinen Leser bewerten ${ }^{208}$. Im Zentrum dieses Werkes stehen vor allem Hinweise für die Ergreifung der richtigen Heilungsmaßnahmen für die Pestkranken, darunter insbesondere die vorzunehmende Behandlung der Pestbeulen.

Dagegen wurde 1535 von den vier geschworenen Augsburger Stadtärzten Adolph Occo, Johannes Tieffenbach, Gereon Sayler und Wolfgang Talhauser ein umfassender Pesttraktat erlassen, welchem unter dem medizinischen Schrifttum über die Pest in Augsburg eine Sonderstellung zukommt ${ }^{209}$. Die Augsburger Doktoren treten darin nämlich als städtische Pestexperten auf, die mit ihrer medizinischen Abhandlung auf Missstände auf dem

\footnotetext{
${ }^{204}$ JUNG, Ain nutzliche trostliche und kurze underrichtung (1) [Augsburg: Silvan Ottmar 1521].

${ }^{205}$ Ebd. S. A II.

206 DERS., Ain nutzliche trostliche und kurze underrichtung (2) [Augsburg: Silvan Ottmar 1535]; DERS., Ain nutzliche trostliche und kurze underrichtung (3) [Augsburg: Silvan Ottmar 1539, Valentin Ottmar 1563; Strassburg: Wendelin d. Ä. Rihel 1535; Innsbruck: Rupprecht Höller 1562].

207 KLAINMÜLLER, Ain hymlischen vnn Natürlichen anzaygung [Augsburg: Hans Schönsberger, 12. Oktober 1521].

${ }^{208}$ Seine Beweggründe zum Verfassen dieser Pestschrift stellte der Autor in der Einleitung folgendermaßen dar, S. A II: Hab ich bedacht umb empfangmer guthait meinen guten günnern und freunden danckpar zu sein in disen schweren zeytten und leüffen unnserer stat Augspurg/ einwonern zu trost und hilff/ ain nutzlichen und trostlichen underrichtung zugeben/wie sich mänigklich in disen erschtockenlichen zeytten und leüffen halten soll.

${ }^{209}$ OCCO/SAYLER/TIEFFENBACH/TALHAUSER, Was die Pestilentz an jr selbs sey [Augsburg: Philipp Ulhart 1535].
} 
Augsburger Gesundheitsmarkt während der Pestzeit hinweisen wollten ${ }^{210}$. Obwohl ihre Pestlehre jedem anzubieten ist, wer klag/ wie doch kain gelerter ehrliebender billicherwey $\beta$ haben mag/ab diesem unserm schreiben hat, wollten sie mit ihrem gelehrten Werk doch vor allen verstendigen/ und sonderlich vor unserer Oberkait/willig und berayt sein ${ }^{211}$. Damit sind vor allem die anderen Augsburger Mediziner und die Bürgermeister mit dem städtischen Rat angesprochen, die gemeinsam zwei Grundziele während der Pestausbrüche anstreben sollten: Eindämmung der Kurpfuscherei auf dem Gesundheitsmarkt in Augsburg und Beachtung der medizinischen Grundsätze von Hippokrates (um 460 v. Chr. - um 370 v. Chr.) und Galen (um 129 n. Chr. - um 216 n. Chr.) in der Behandlung der Pestkranken.

In der Vorrede ihrer Abhandlung erklären die Doktoren ausführlich, warum sie bisher noch keine Pestschrift für den gemainen man drucken ließen und welche Gründe sie jetzt dazu veranlassten. Nach ihrer Überzeugung war es bislang ihrerseits nicht nötig, die Pestlehre schriftlich mitzuteilen, weil in Augsburg schon längst zahlreiche Pestschriften von hochgelehrten Ärzten wie z.B. Ambrosius Jung erschienen seien und jedermann daraus viel über die Pest lernen könne. Trotzdem handeln ihrer Ansicht nach etliche Leute gar leichtsinnig und so an kainerlay getrewer underrich benugig/ allain zum verspotten und affterreden genaigt seind/ solchs und anders unvernünfftigklich verachten ${ }^{212}$. Die meisten Verfehlungen betrafen dabei den Gebrauch von Arzneien, was die Stadtärzte auch bei allen anderen Krankheiten ständig beobachteten. Wenn sich solche Missstände nur aus der reinen Unwissenheit der Leute ergäben, ertrügen es die Mediziner geduldig, weil der unverstand des gemainen mans zu mehrern thail dieser zeyt ungewarnet will sein/dann durch fürwitz will ain yeder sein weys behalten ${ }^{213}$. Solches Vergehen entstünde aber in Augsburg gerade dadurch, dass einige Betrüger den einfältigen und unverständigen Leuten viele Arzneien anpriesen, die sie weder aufgrund glaubwürdiger Schriften noch gesammelter Erfahrung herstellten, weshalb sie den Kranken große Schäden an Leib und Leben zufügen könnten. Dabei sollte man auch bei der Pestkrankheit die Anwendung innerlich wirkender Medikamente immer von der entsprechenden Behandlung der Beulen begleiten lassen. Daher sähen sie sich als Augsburger Stadtärzte aufgrund ihrer Verpflichtungen, die sie der städtischen Obrigkeit schuldig seien, dazu veranlasst, solche Missstände nicht länger $\mathrm{zu}$ dulden und einen Bericht darüber zusammenzustellen.

\footnotetext{
${ }^{210}$ Der Untertitel dieser Pestschrift lautet: Mit kurtzer widerlegung erlicher grober einfallender irsal/ so sich bey uns wider der alten schrifft und gegründete ursachen zutragen.

${ }^{211}$ Ebd. S. A IIv.

212 Ebd. S. A II

${ }^{213}$ Ebd. S. A IIv.
} 
Die Doktoren riefen in ihrer Schrift alle Stadtbewohner dazu auf, dass sie sich mutwilliger unverstandner fürwitz/ solcher gewinnsichtiger und newgelerten artzet/ unter denen auch vil far ungelernt landstreicher/ mussig standen ${ }^{214}$. Bei einer Pesterkrankung sollten sich die Menschen zum Zwecke der Behandlung und Hilfeleistung nur an jene Ärzte wenden, die umb alle ire gethone hendel wissen/ und künnen genugsame und glaubwirdige verantwurtung darthun ${ }^{215}$.

In den medizinischen Grundlagen ihres Traktates stützten sie sich nicht auff ettlich new practiken und unansechlich lerer/ sonder auff die eltesten/ als Hyppocratem und Galenum/ auch ander so inen nachgevolgt ${ }^{216}$. Für die Augsburger Stadtärzte galten nämlich Hippokrates und Galen als die höchsten Autoritäten in der Medizin. Sie stellten jedoch fest, dass deren Schriften in letzter Zeit weniger studiert würden, was sie mit dem vorreformatorischen Zustand in der Theologie verglichen. Man bevorzuge nämlich auf der Kanzel und in der Schule die späteren Autoren und vergesse dabei die Bibel bzw. die alten Autoritäten. Dasselbe geschehe in der Medizinlehre, wo man mit aines Arabischen Machumetischen schreibers gethon auch den maisten thail mit ettlicher newer Welschen schreyber Kreüter buchlen/ und geschwätzigen practiken und disputieren umbgangen/ und hat daneben Hippocratem, Galenum, Dioscoridem unter der banck lassen ligen/ die man doch fürnaemlich hat wissen und lesen sollen ${ }^{217}$.

Aus diesem Grund versahen die Doktoren ihr Traktat reichlich mit gelehrten Ausführungen und Marginalglossen zu den Werken von Galen und Hippokrates, weil sie wussten, das dem gemainen hauffen artzt der Galenus unbekannt ist/ und deren etlich nit wissen in welchem buch er von disem oder jhenen schreibt/ vor den gelerten ärzten bedörfft es sich nit ${ }^{218}$. Auf diese Weise beanspruchten die Stadtärzte, andere studierte Mediziner dazu anzuleiten, dass sie ihre Heilkunst vornehmlich auf die ältesten medizinischen Autoritäten und nicht nur auf deren spätere Nachfolger stützen sollten.

Darüber hinaus wollten sie ebenso die Augsburger Wundärzte, zu deren Aufgaben die Behandlung der äußeren Pestzeichen gehörte, in der Pestlehre unterweisen. Im Abschnitt über die Beulen und Blattern ermahnen die Doktoren daher ausdrücklich dazu, die Pestbeulen nicht so spät wie andere Arten von Abszessen zu öffnen. Die Stadtärzte konstatierten nämlich, dass die Wundärzte darin in der Heilkunst viele Fehler verüben. Diese Unzulänglichkeiten in der

\footnotetext{
${ }^{214}$ Ebd.

215 Ebd.

${ }^{216}$ Ebd. S. F IIIv.

${ }^{217}$ Ebd. S. A IVv.

${ }^{218}$ Ebd. S. D IIv.
} 
Wundarznei entstünden vornehmlich dadurch, dass nit ain yeder die erfarung/ unnd der wenigest thail etwas gelesen hab ${ }^{219}$.

Aus diesem Grund richteten sie einen direkten Aufruf an die Augsburger Wundärzte: derhalb unser fleissig bitt/ ain yeder dem es zugehört/ wolle dises mit fleyß lesen/ und gedencken was er seinen ehren/Got und dem nächsten schuldig sey/ Nämlich das er dem nächsten diene wie er wolt das ime mit aller vernunfft und kunst gedienet wurde. Derhalben wöllen wir wenig/ und das grund in der schrifft ursachen und erfarung hat/ schreiben/ damit mans dest baß mercken/ und sich darnach richten kündel unnd das alles au $\beta$ dem grund Galeni und Hippocratis $^{220}$. Die von den vier Augsburger Doktoren aufgezeichnete Pestschrift war also auch als Lehrbuch für alle anderen Mediziner konzipiert, mit dessen Hilfe sich diese die auf den Grundsätzen von Hippokrates und Galen basierende Pestlehre aneignen sollten. Durch die Drucklegung ihres Pesttraktates wollten die Augsburger Stadtärzte daher sowohl ihren Expertenanspruch unter allen Medizinern öffentlich hervorheben als auch die städtische Obrigkeit zu weiteren Maßnahmen gegen die Kurpfuscher auffordern.

Während der Pestepidemie von 1547 wurde vermutlich auch ein Pesttraktat des Augsburger Stadtarztes Wolfgang Talhauser zum Druck gelegt. Darüber berichtet Johannes Vogt der Jüngere aus Wien in der Vorrede zu der von ihm vorbereiteten Ausgabe der Pestschrift von Doktor Matthias Böham von Neuhaus ${ }^{221}$. Talhauser war dabei nicht der einzige Autor seines medizinischen Werkes, sondern verbesserte und ergänzte lediglich die früheren Werkausgaben von Böham ${ }^{222}$. Zur erneuten Drucklegung dieses Buches, dessen Exemplare in Augsburg nicht mehr erhältlich waren, bewegten Talhauser vor allem die darin enthaltenen ausführlichen Erörterungen über die rechtzeitige Erkennung des pestilenzischen Fiebers. Der direkte Anlass dafür waren nämlich die 1547 ausbrechenden Meinungsverschiedenheiten unter den gelehrten Ärzten, ob das zu Augspurg/der Gebrechen/

\footnotetext{
${ }^{219}$ Ebd. S. F I.

${ }^{220}$ Ebd.

${ }^{221}$ BÖHAM, Ain nutzliche/ vnnd für den gemainen Man/ genugsam gegründte vnderricht [Augsburg: gedruckt von Philipp Ulhart und zum Neudruck aufgelegt von Johannes Vogt dem Jüngeren um 1570; bearbeitet und ergänzt von Wolfgang Talhauser um 1547]. Zur Datierung des Werkes von Talhauser dient sowohl der folgende Hinweis in der Vorrede von Johannes Vogt dem Jüngeren, S. A Iv: vor etlich und zwaintzig jarn zu Augspurg nach dem Schmalkaldischen Krieg gebessert/ und wider zu Trucken verordnet durch D. Wolfgang Talhauser/ in grosser not allenthalb dazumal sterbender leüff halber getruckt wie auch die auf dem nächsten Blatt abgedruckte Vorrede von Talhauser zu seiner Ausgabe der Pestschrift.

${ }^{222}$ Aufgrund der Informationen in der Vorrede von Johannes Vogt dem Jüngeren und in der von Talhauser kann man die folgenden früheren Druckausgaben dieses Textes festlegen: um 1529 - verfasst und gedruckt in Wittenberg von Doktor Matthias Böham vom Neuhaus; um 1540 - Ausgabe von Doktor Johannes Vogt (Vater von Johannes Vogt dem Jüngeren, der Pestarzt in Ulm).
} 
daran doch mancher stirbt/ nicht die rechte Pestilentz müg genennet werden ${ }^{223}$. Die rechtzeitige Pestdiagnose wurde damals insbesondere dadurch erschwert, dass besonders viele Kranke in der Anfangsphase der Krankheit noch keine sichtbaren Pestbeulen im Körper hatten. Dann aber stellte sich endgültig heraus, dass es sich um eine Pestepidemie handelte. Daher beschloss Talhauser, dieses Pestbuch zu veröffentlichen, darmit sich gemain man/ so in der Ertzney nit gestudiert hat/ dieweil wisse zuhalten/ biß mit der zeyt die gelerten der sachen ains werden/ was allhie für ain Sucht undter dem gemainen man umbgange, (...) ehe das die Sucht iren rechten namen bey den geleerten überkommel dan es wurde sunst dieweil manchem zu kurtz geschehen ${ }^{224}$.

Die zögerliche Aufnahme der notwendigen Behandlung bis zu dem Zeitpunkt, an dem die ersten äußeren Krankheitszeichen am Körper auftraten, bedeutete nicht nur geringere Chancen für die Genesung der Kranken, sondern führte auch dazu, dass ainer nach dem andern vergyfftet wirdt. Außerdem konnten auch viele Personen, wie es nun gnug in erfarnuß ist, an der Pest sterben, denen gar kain zaichen auffert ${ }^{225}$. Aus diesem Grund war für Talhauser die rechtzeitige Selbstdiagnose vor dem Auftreten der äußeren Pestzeichen von größter Bedeutung. Demzufolge finden sich schon im ersten Kapitel dieser Pestschrift genaue Erläuterungen zur erkandtnus des pestilentzischen Fiebers mit allen zaichen und umbständen/ verstendiger/ weder vor/ beschriben wirt/ damit ain yeder wisse/ so bald er sich beschwäret befindet/ ob ettwas von solchem Fieber an im sey oder nit ${ }^{226}$.

Mit Hilfe einer derartigen Unterweisung über die Beschaffenheit des „pestilenzischen Fiebers“" und die anderen frühesten Pestsymptome sollten alle Augsburger Stadtbewohner dazu befähigt werden, leichter eigene Erkrankungen $\mathrm{zu}$ erkennen und sofort danach die notwendige Behandlung aufzunehmen. Zu diesem Zweck wurden zwei Holzschnitte mit gründlichen Ausführungen über die Pestbeulen hinzugefügt (Abb. 11), die vermutlich Talhauser selbst erarbeitete. Der Werkausgabe von Talhauser liegt also in erster Linie die Idee eines medizinischen Werkes zur besseren Selbstdiagnose der Pest zugrunde.

\footnotetext{
${ }^{223}$ BÖHAM, Ain nutzliche/ vnnd für den gemainen Man/ genugsam gegründte vnderricht [Augsburg: gedruckt von Philipp Ulhart und zum Neudruck aufgelegt von Johannes Vogt dem Jüngeren um 1570; bearbeitet und ergänzt von Wolfgang Talhauser um 1547], S. A II.

${ }^{224}$ Ebd.

${ }^{225}$ Ebd. S. A IIv.

${ }^{226}$ Ebd. S. A II-IIv.
} 


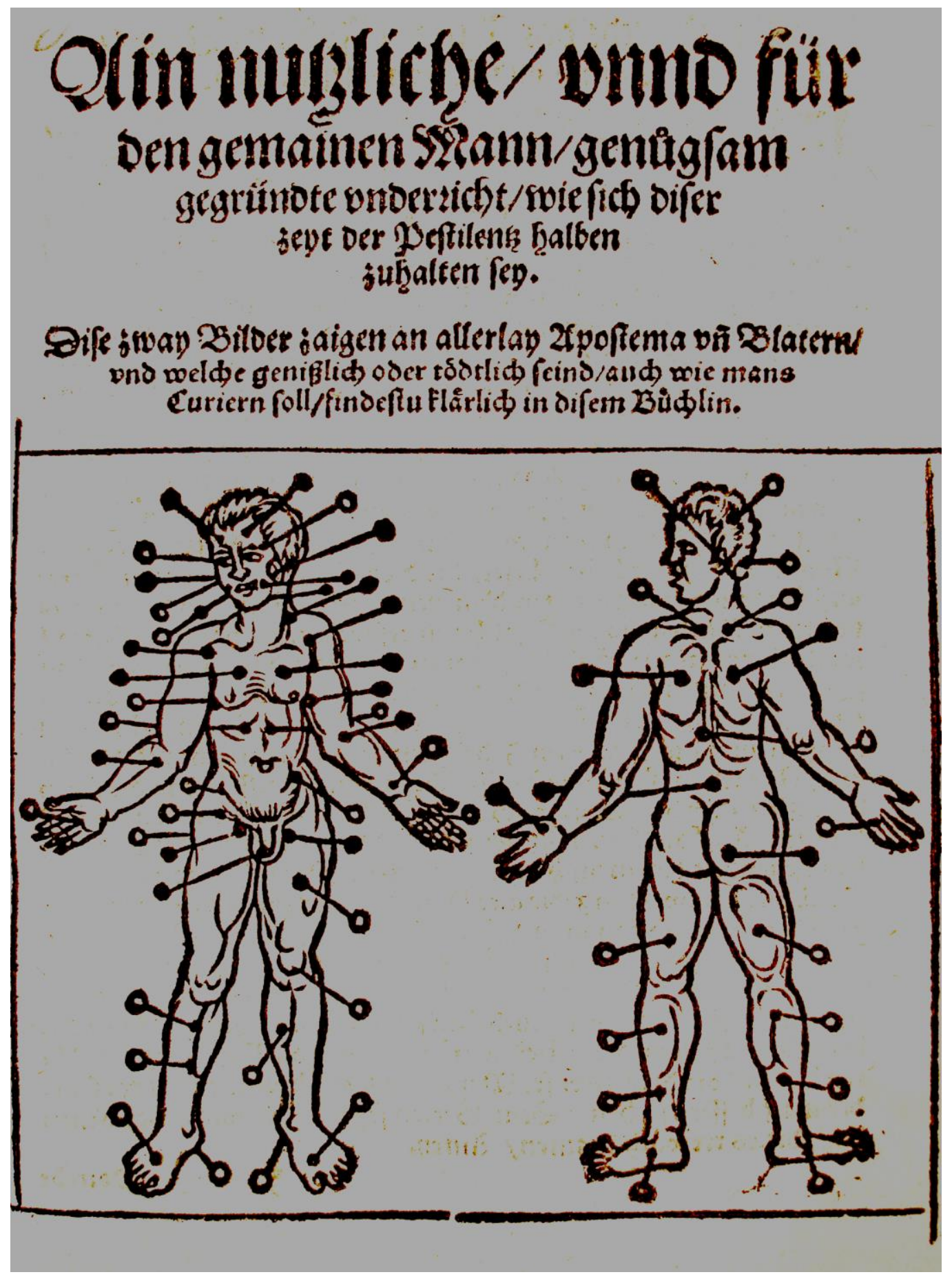

Abb. 11: Darstellung der tödlichen und heilbaren Apostemata und Blattern bei einem Pestkranken, in: BÖHAM, Ain nutzliche/ vnnd für den gemainen Man/ genugsam gegründte vnderricht [Augsburg: gedruckt von Philipp Ulhart und zum Neudruck aufgelegt von Johannes Vogt dem Jüngeren um 1570; bearbeitet und ergänzt von Wolfgang Talhauser um 1547] 


\section{Augsburg als Druckort für die Pestschriften auswärtiger Autoren}

Außer den Werken der Augsburger Doktoren wurden auch zahlreiche Pestschriften von auswärtigen akademischen Ärzten in Augsburg gedruckt, die schwerpunktmäßig in den Dreißiger Jahren des 16. Jahrhunderts erschienen. Im Jahr 1533 publizierten hier zwei Doktoren aus Ingolstadt: Johannes Agricola $^{227}$ und Michael Fencken, Ordinarius an der Universität zu Ingolstadt ${ }^{228}$, ihre medizinischen Pestwerke. Auch zwei Memminger Ärzte, Doktor Adam Zwicker ${ }^{229}$ und Doktor Ulrich Wolfhart ${ }^{230}$, ließen 1537 und um 1540 ihre Pestschriften in Augsburg auflegen. Außerdem erschienen zwei Pestregimente von Doktoren aus den größten Nachbarstädten: 1520 von Johannes Stocker, einem Ulmer Stadtarzt ${ }^{231}$ und um 1530 von Doktor Johannes Magenbuch aus Nürnberg ${ }^{232} .1522$ druckte man in Augsburg ein Traktat von dem damals in München tätigen Doktor Alexander Seitz ${ }^{233}$ und 1533 eine Pestschrift von Marcus Deas Veringer, Stadtarzt in Neumarkt ${ }^{234}$. Dagegen wurden im Jahr 1531 sowohl ein Regimen sanitatis mit einem Kapitel über die Pestlehre von Luis Lobera de Avila in der deutschen Übersetzung von Doktor Michael Krautwadel aus Landsberg ${ }^{235}$ als auch ein Arzneibuch mit einigen Rezepten für Medikamente gegen die Pest vom bischöflichen Augenarzt zu Dillingen Bartholomeus Vogter ${ }^{236}$ herausgegeben. Darüber hinaus wurden in Augsburg auch die Werke von Ärzten aus weiter entfernt gelegenen Städten aufgelegt: 1512 ein kleines Pestbuch von Andreas von Überlingen ${ }^{237}, 1518$ ein Pesttraktat von Doktor Johannes Engel aus Wien ${ }^{238}, 1525$ ein anonymes Werk, das vom Autor seinen Herren und Bürgern in Zürich gewidmet wurde ${ }^{239}$ und 1534 eine Pestschrift von Doktor Michael Milchtaler aus Schwaz ${ }^{240}$. Augsburg als Druckort und Buchmarkt für medizinische Texte hatte also auch bei auswärtigen Ärzten einen exzellenten Ruf.

\footnotetext{
${ }^{227}$ AGRICOLA, Ain grüntlicher fleissiger außzug [Augsburg: Philipp Ulhart, 10. März 1533].

${ }^{228}$ FENCKEN, Ain gut nützlich Regiment [Augsburg: Alexander Weißenhorn 1533].

${ }^{229}$ ZWICKER, Ein kurtze wolgegründte underrichtung [Augsburg: Silvan Ottmar 1537].

${ }^{230}$ WOLFHART, Ain kurtzer Bericht [Augsburg: Philipp Ulhart um 1540].

${ }^{231}$ STOCKER, Ain Regiment für die Pestilentz [Augsburg: Sigmund Grimm/Marx Wirsung 1520].

${ }^{232}$ MAGENBUCH, Ain nützlichs vnd Bewaerts gemains Regiment [Augsburg: Philipp Ulhart d. Ä. um 1530].

${ }^{233}$ SEITZ, Ain schoener Tractat von dem Saturnischen gschoß der Pestilentz [Augsburg: Otmar Silvan, 15. Februar 1522].

${ }^{234}$ VERINGER, Ein kurtz nützlichs Regiment [Augsburg: Heinrich Steiner 1533].

${ }^{235}$ LOBERA DE AVILA/KRAUTWADEL, Ein nutzlich Regiment der gesundtheyt [Augsburg: Heinrich Steiner 1531].

${ }^{236}$ VOGTER, Ajn nutzlich vnnd notwendigs Artzney Buechlin [Augsburg: Philipp Ulhart 1531], S. 66-67.

237 ANDREAS VON ÜBERLINGEN, Pestilentzbůchlin [Augsburg: Johann Otmar, 27. Oktober 1512].

${ }^{238}$ ENGEL, Tractat von der Pestilentz [Augsburg: Sigmund Grimm und Marx Wirsung, 4. November 1518].

${ }^{239}$ ANONYMUS, Ain regiment wider Die Pestilentz [Augsburg: Johannes Schönsberger um 1525].

${ }^{240}$ MilCHTALER, Ain Kurtz vnd notwendig vnderricht vnd Regiment [Augsburg: Heinrich Steiner 1534].
} 


\section{Lesepublikum der medizinischen Buchdrucke}

In allen Augsburger Druckereien wurden bis zum Jahre 1555 insgesamt 174 medizinische Schriften aller Art gedruckt, was 39,6\% der gesamten Buchproduktion im Bereich des "naturwissenschaftlichen" Schrifttums in Augsburg entspricht ${ }^{241}$. Darunter bildete die Medizin die wichtigste Untergattung. Die Augsburger Drucker veröffentlichten nur selten lateinische Werke in Eigeninitiative, weshalb deren Anteil bei knapp zehn Prozent aller „Naturwissenschaften“ liegt. Daraus ist ersichtlich, dass das Programm der Augsburger Offizinen primär auf Bücher zur Wissensvermittlung für den Laien fokussiert war.

Gleiches gilt auch für die Drucklegung der Augsburger Pestschriften. Abgesehen von einigen lateinischen bzw. solchen Texten, die gleichzeitig für ein Fach- und Laienpublikum vorgesehen waren ${ }^{242}$, ist in den deutschsprachigen Pestdrucken bis 1550 vorrangig der gemeine man als erwünschter Leserkreis angegeben. Das Hauptziel der gedruckten Pestschriften bestand also darin, breiten Bevölkerungsschichten die notwendige Aufklärung über die Seuche zu vermitteln, weil solich artzney und hilff dem gemaynen man verborgen und unwyssent bleibt ${ }^{243}$. Daher stellten die Autoren die Grundsätze ihrer Pestlehre zusammen auf das kürzest/ damit man sich/ sovil natürlich ist/ müg verhieten ${ }^{244}$. Darüber hinaus kann man insbesondere in der ersten Hälfte des 16. Jahrhunderts bei den Autoren und Druckern eine größere Sorgfältigkeit feststellen, wenn es um die übersichtlichere Strukturierung und bessere Verständlichkeit ihrer Bücher ging. Mittels überschaubarer Register, Kapitalüberschriften oder gedruckter Marginalien mit kurzen Inhaltsangaben wollten sie die

\footnotetext{
${ }^{241}$ KÜNAST, Buchdruck, S. 246-247.

${ }^{242}$ Vor allem OCCO/SAYLER/TIEFFENBACH/TALHAUSER, Was die Pestilentz an jr selbs sey [Augsburg: Philipp Ulhart 1535]. Auch in STEINHÖWEL, Büchlin der ordnung [Ulm: Johannes Zainer von Reutlingen, 11. Januar 1473] wird die Lektüre des Werkes auch jungern willigen meister der scherer, die noch nit gantz in wund ertzney geubet synd empfohlen; Ähnlich in SEITZ, Ain schoener Tractat von dem Saturnischen gschoß der Pestilentz [Augsburg: Otmar Silvan, 15. Februar 1522] werden einige Kapitel direkt an das gelehrte Lesepublikum gerichtet, S. 150: In diesem capitel/ beger ich nit leer zu geben/ den wonwitzigen/ keibstrettigen koepffen/ aber disen/ wie ir/ die mit menschlicher art bas erleücht sein/ darumb wissen und glauben entlich etlich lobsam artickel von den hochgelerten grüntlich beschriben; S. 195: Das bevilh ich dem barbierer/ aber die warhait zu sagen/würt dem menschen/zu gfaerlichem tod herinn vil geirt/ und in zwen weg.

${ }^{243}$ ANONYMUS, Ain regiment wider Die Pestilentz [Augsburg: Johannes Schönsberger um 1525], S. A Iv.

244 JUNG, Ain nutzliche trostliche und kurze underrichtung (1) [Augsburg: Silvan Ottmar 1521], S. A II. Die ähnlichen Aussagen auch z.B. in KLAINMÜLLER, Ain hymlischen vnn Natürlichen anzaygung [Augsburg: Hans Schönsberger, 12. Oktober 1521], S. C IV: ich hett im willen vill meer darvon zu schreyben/ ich besorg aber/ es möchte den lesern verdrießligkait bringen oder in FENCKEN, Ain gut nützlich Regiment [Augsburg: Alexander Weißenhorn 1533], S. A IIv: in fünff sondere Capitel getailet/ welliche ich kürtzlich/als vil die materi erleyden mag/ schlechtlich/ gemainlich/ nicht mit scharpfer/ tapffer/ verborgner schrifft/ oder vil allegationen/ dem gemainenen mann zu gut.
} 
Zugänglichkeit und den Gebrauch derartiger Bücher erhöhen, um dadurch ein möglichst breites Publikum zu erreichen ${ }^{245}$.

Um Auskunft über die üblichen Preise für Pestschriften geben zu können, kann leider nicht auf Preislisten o.ä. Quellenmaterial zurückgegriffen werden, da solches nicht überliefert ist. Man kann aber aufgrund der von Georg Krapff, dem Ingolstädter Buchbinder und -händler, erhaltenen Rechnungen über seine Einkäufe in Augsburg bei dem Großsortimenter Wolfgang Präunlein einige Einblicke in die Preise der medizinischen Schriften gewinnen ${ }^{246}$. Aus seinen Rechnungen für die Jahre 1529-1530 geht nämlich hervor, dass er für gedruckte Arzneibücher folgende Summen ausgeben musste: 6 Artznei fleugauß - 4 Schilling, 1 Arznei buchel schoner - 2 Kreuzer und 4 artznei buchlen - 1 Schilling. Im Durchschnitt bezahlte Krapff also zwischen einem bis zu drei Kreuzern pro Arzneibuch ${ }^{247}$. Dabei muss man aber unterstreichen, dass es sich hier um die Buchpreise aus dem Zwischenhandel handelt, in denen keine Buchbinderkosten sowie der zusätzliche Gewinn aus dem Buchhandel miteingerechnet waren. Um die erforderlichen Ausgaben der Zeitgenossen für den Einkauf einer gedruckten Pestschrift einschätzen zu können, ist es auch notwendig, die Preise für die überlieferten Buchdrucke in Relation zu ihrem Umfang zu stellen. Zu diesem Zweck ist die Zusammenstellung von Hans-Jörg Künast für einige Druckschriften aus den Jahren 14661536 sehr aufschlussreich, in der die Endabnehmerpreise und die Kosten aus dem Zwischenhandel pro Druckbogen miteinander verglichen wurden ${ }^{248}$. Die Preise pro Bogen im Zwischenhandel betragen demnach zwischen 0,23 bis 0,90 Pfennig und nur für ein Werk belaufen sich die Kosten auf 6,67 Pfennige pro Bogen. Die Endabnehmerpreise für die billigsten Druckschriften liegen dagegen bei 1,5 bis 2,1 Pfennigen pro Bogen, wobei für das teuerste gedruckte Buch pro Bogen 16,4 Pfennige gezahlt werden mussten.

Weil die Pestschriften in der ersten Hälfte des 16. Jahrhunderts vor allem für den gemeinen man vorgesehen waren und anscheinend auch preiswert gedruckt werden konnten, ist von ca. zwei Pfennigen pro Bogen für solche Werke auszugehen. Bei einem Umfang zwischen 10 und 20 Foliobogen war eine Druckschrift von der Pestlehre für ungefähr 20 bis 40 Pfennige (5 bis 10 Kreuzer) im Endhandel erhältlich. Die medizinischen Einblattdrucke konnten dagegen für einige wenige Pfennige von den Zeitgenossen angeschafft werden.

\footnotetext{
${ }^{245}$ Vgl. FEUERSTEIN, Im Druck, S. 32.

${ }^{246}$ KÜNAST, Buchdruck, S. 190-192.

${ }^{247}$ Diese Rechnungen sind nach zwei spätmittelalterlichen Münzensystemen zusammengestellt, die in der ersten Hälfte des 16. Jahrhunderts noch gleichzeitig in Augsburg gebraucht wurden: nach dem Pfund (1 Pfund = 20 Schilling = 240 Pfennige) und nach der im Jahr 1396 eingeführten Leitwährung Gulden (1 Gulden = 60 Kreuzer). Das Verhältnis der Recheneinheiten stellt sich in diesen Rechnungen folgendermaßen dar: 1 Gulden $=$ 20 Schilling $=60$ Kreuzer = 240 Pfennige. Dazu vgl. in: GEFFCKEN, Münze(n), S- 670.

${ }^{248}$ KÜNAST, Buchdruck, S. 193-195.
} 
In diesem Zusammenhang ist ein kurzer Blick auf die damaligen Einkunftsmöglichkeiten in Augsburg sehr hilfreich. Die Durchschnittsgehälter der einfachen Tagelöhner, die in den Quellen als Recher bezeichnet werden, weil sie mit ihrer rechten Hand arbeiten, beliefen sich in dieser Zeit auf etwa 6 bis 10 Kreuzer pro Tag ${ }^{249}$. Für den Kauf eines preiswerteren Pestdruckes musste ein Tagelöhner demzufolge einmalig sein tägliches Durchschnittseinkommen aufwenden. Hinsichtlich eines solchen Verhältnisses zwischen Einkünften und Buchpreisen scheint es durchaus möglich, dass auch die städtischen Unterschichten finanziell imstande waren, derartige medizinische Gebrauchsschriften anzukaufen. Dabei stand den Zeitgenossen eine große Auswahl von gedruckten Pestschriften zur Verfügung, die in verschiedenen Druckformen von Einblattdrucken, über Regimina sanitatis mit dem Abschnitt über die Pest bis hin zu selbständigen Werken über die Pestlehre auf dem Augsburger Buchmarkt angeboten wurden. Die Stadtbewohner konnten daher für ihre Vermögensverhältnisse oder ihr Bildungsniveau geeignete Druckschriften erwerben.

Auf eine breite Rezeption der Pestschriften in Augsburg weisen auch die Wiederauflagen einiger Bücher hin. Dabei wurden viele Werke nicht ausschließlich wegen des Seuchenausbruches in den Jahren 1494, 1521, 1535 oder 1547 in Augsburg gedruckt, sondern sie erschienen häufig auch, wenn die Pest nur in anderen Orten präsent war. Diese Gegebenheiten bestätigen die große Beliebtheit medizinischer Ratgeber und lassen auf ein relativ großes Lesepublikum derartiger Druckschriften schließen.

\footnotetext{
${ }^{249}$ KeLLENBENZ, Wirtschaftsleben, S. 295-297.
} 


\section{Grundsätze der medizinischen Pestlehre}

In Augsburg lässt sich seit den Siebziger Jahren des 15. Jahrhunderts ein umfangreiches medizinisches Gebrauchsschrifttum nachweisen, auf das die Zeitgenossen zugreifen konnten. Dieser Umstand begünstigte die Verbreitung der Medizinlehre unter der Augsburger Stadtbevölkerung. Im Zusammenhang damit stellt sich im Folgenden die Frage, welchen Diskurs über die Pest die damaligen Mediziner in ihren Werken vermitteln konnten. Anhand der oben dargestellten Pestschriften wird erläutert, aufgrund welcher Denkformen und Wahrnehmungen die medizinische Pestlehre bis zur Mitte des 16. Jahrhunderts formuliert wurde. Der Schwerpunkt der Analyse liegt dabei vor allem auf den Traktaten der Augsburger Doktoren, die die städtische Pestbewältigung in Augsburg entscheidend mitprägten. Bei einer detaillierten Auswertung der Pestschriften lässt sich konstatieren, dass diese Gebrauchsschriften bei Form und Einteilung des Textstoffes weitgehende Gemeinsamkeiten aufweisen. Abgesehen von den medizinischen Pestblättern, die in kurzer und einheitlicher Form einen grundsätzlichen Maßnahmenkatalog gegen die Pest präsentieren, kann man in den meisten Pestschriften deutlich drei getrennte Grundteile über die Ätiologie, Prophylaxe und Therapie unterscheiden. Zuerst werden fast immer theoretische Aspekte über die Ursachen der Pest behandelt, die jedoch in den praxisorientierten Texten auf die wichtigsten Aspekte beschränkt bleiben ${ }^{250}$ oder lediglich in den Kapiteln über die medizinischen Maßnahmen kurz eingestreut werden ${ }^{251}$. Dann folgen Anweisungen zur Prophylaxe in der Pestzeit und zuletzt zur Therapie der Pestkranken. Obwohl die Größe der einzelnen Teile abhängig von den Absichten der Autoren manchmal sogar beträchtlich variieren kann, wurden alle Pestwerke gewöhnlich nach demselben Gliederungsmuster zusammengestellt.

\footnotetext{
${ }^{250}$ Hier z.B. alle Ausgaben der Pestschriften von Ambrosius Jung aus dem 16. Jahrhundert: JuNG, Ain nutzliche trostliche und kurze underrichtung (1-3) [Augsburg: Silvan Ottmar 1521, 1535, 1539; Valentin Ottmar 1563]; auch BÖHAM, Ain nutzliche/ vnnd für den gemainen Man/ genugsam gegründte vnderricht [Augsburg: Philipp Ulhart, bearbeitet und ergänzt von Johannes Vogt dem Jüngeren um 1570].

251 ANONYMus, Ain regiment wider Die Pestilentz [Augsburg: Johannes Schönsberger um 1525] oder WOLFHART, Ain kurtzer Bericht [Augsburg: Philipp Ulhart um 1540].
} 


\section{Das antike Medizinkonzept: Hippokrates, Galen und ihre arabischen Nachfolger}

In allen ausgewerteten Pestschriften kann man generell feststellen, dass sie ausnahmslos auf den gleichen medizinischen Grundsätzen basieren. Als theoretische Grundlage der damaligen Pestlehre galt das antike Medizinkonzept von Hippokrates und Galen. Daneben waren auch ihre arabischen Nachfolger von großer Bedeutung, unter denen Avicenna (arab. Ibn Sīnā, 980 - 1037) mit seinem häufig zitierten Werk über die Fieberlehre (Canon, Lib. IV, Fen I) der wichtigste Platz zukam. In einigen Pestschriften beziehen sich die Autoren direkt auf ihre jeweiligen medizinischen Autoritäten, wodurch sich zwei allgemeine Tendenzen erkennen lassen. Zum Ersten ist die Gruppe von Texten zu unterscheiden, deren Autoren sich in ihren Ausführungen schwerpunktmäßig auf die ältesten Mediziner beriefen, von denen aber bevorzugt Galen angeführt wurde ${ }^{252}$. Die zweite Gruppe von Autoren bevorzugte dagegen das medizinische Oeuvre von Avicenna, der von ihnen zuweilen als „Fürst der Ärzte“ gepriesen wurde ${ }^{253}$. Neben Avicenna sind in den Augsburger Pestschriften noch vereinzelte Bezüge auf zwei andere arabischen Autoren, Rhazes (um 864 - 925) und Albumasar (um 787 - 886) anzutreffen ${ }^{254}$.

Dabei ist anzumerken, dass bis zur Mitte des 16. Jahrhunderts das Schrifttum von Avicenna unter den gelehrten Ärzten breiter rezipiert wurde als die Werke antiker Mediziner. Auf diesen Zustand weist schon Arnold Klebs in seiner Untersuchung über die Pestinkunabeln $\operatorname{hin}^{255}$. Diese Bewertung stimmt aber auch weitgehend mit der zeitgenössischen Wahrnehmung, die Rezeption des medizinischen Wissens betreffend, überein. Die Augsburger Stadtärzte wiesen nämlich in ihrer Pestschrift darauf hin, dass dem gemainen hauffen artzt der Galenus unbekannt ist oder dass in der artzney ist man etwan mit aines Arabischen Machumerischen schreibers gethon ${ }^{256}$.

\footnotetext{
${ }^{252}$ Hier vor allem OCCO/SAYLER/TiefFENBACH/TALHAUSER, Was die Pestilentz an jr selbs sey [Augsburg: Philipp Ulhart 1535]; Auch in AGRICOLA, Ain grüntlicher fleissiger außzug, [Augsburg: Philipp Ulhart 10. März, 1533]; VERINGER, Ein kurtz nützlichs Regiment, [Augsburg: Heinrich Steiner 1533]; WOLFHART, Ain kurtzer Bericht [Augsburg: Philipp Ulhart um 1540].

${ }^{253}$ STEINHÖWEL, Büchlin der ordnung [Ulm: Johannes Zainer von Reutlingen, 11. Januar 1473]; KLAINMÜLLER, Ain hymlischen vnn Natürlichen anzaygung [Augsburg: Hans Schönsberger, 12. Oktober 1521]; ENGEL, Tractat von der Pestilentz Joanni [Augsburg: Sigmund Grimm und Marx Wirsung 4. November 1518]; FenCKEN, Ain gut nützlich Regiment [Augsburg: Alexander Weißenhorn 1533]; SEITZ, Ain schoener Tractat von dem Saturnischen gschoß der Pestilentz [Augsburg: Otmar Silvan, 15. Februar 1522].

${ }^{254}$ Dagegen werden im ersten gedruckten deutschsprachigen Pestbuch von Steinhöwel noch andere arabische Autoren kurz erwähnt: Hali ibn Ridwan, Averroes, Serapion, Mesue und Isaac. Vgl. SudHOFF, Der Ulmer Stadtarzt, S. 204-205.

${ }^{255}$ KLEBS, Geschichtliche Untersuchungen, S. 86, 98.

${ }^{256}$ OCCO/SaYler/TiefFenbach/TalHauser, Was die Pestilentz an jr selbs sey [Augsburg: Philipp Ulhart 1535], S. D IIv.
} 
Trotz dieser Differenzen muss aber betont werden, dass eine deutliche Bevorzugung von antiken oder arabischen Gelehrten für die Grundsätze der Pestlehre keine gewichtigen Konsequenzen hatte ${ }^{257}$. In beiden Fällen blieben die medizinischen Prinzipien immer gleich. Außerdem wurden bisweilen die arabischen und antiken Gelehrten parallel herangezogen und nebeneinander zitiert. Die Augsburger Stadtärzte, deren Pestschrift vornehmlich auf den ältesten medizinischen Autoritäten basiert und mit reichlich Zitaten von Galen und Hippokrates versehen ist, lehnten das Schrifttum der arabischen Mediziner keinesfalls kategorisch $\mathrm{ab}$, sondern beriefen sich auch mehrmals auf das Werk von Avicenna. Von den zeitgenössischen Medizinern schätzten sie dagegen Johannes Manardus (1462-1536) aus Ferrara sehr, der hochgelert artzet zu unsern zeyten/der in allen künsten und sprachen hoch erfarn $^{258}$, wie auch ihren achtbarn hochgelerten (...) herren und guten freünd Ambrosius Jung $^{259}$. In den Augsburger Pestschriften sind ebenfalls einige kurze Bezüge auf andere antike Denker wie z.B. Platon, Aristoteles, Augustinus, Ptolemäus, Dioscorides oder Alexander Benedictus anzutreffen, die aber meistens nur in den akademischen Ausführungen zum Thema Astronomie kurz Erwähnung finden.

Im Allgemeinen ist also in allen Pestschriften eine starke Anlehnung an die Tradition zu konstatieren, deren Quelle aus der galenisch-hippokratischen Medizin entspringt. Diesem altertümlichen Krankheits- und Gesundheitskonzept liegt die Vorstellung zugrunde, dass sich die Krankheitsursachen vornehmlich in den flüssigen Substanzen des Körpers befänden ${ }^{260}$. Die körperliche Beschaffenheit des Menschen besteht demnach aus vier Säften (Humores): Blut, Schleim, gelbe und schwarze Galle, denen vier Elemente: Luft, Wasser, Feuer und Erde entsprechen und die demgemäß die primären Qualitäten Feuchtigkeit, Kälte, Wärme und Trockenheit besitzen. Diese Flüssigkeiten werden, so die Vorstellung, von Natur aus in einem dauerhaften Mischungsverhältnis für jedes Individuum festgelegt, wodurch die körperliche Konstitution eines Menschen grundlegend bestimmt ist. Abhängig von den jeweils aus der individuellen Zusammensetzung der Körpersäften hervorgehenden Qualitäten kann man vier grundsätzliche Menschentypen unterscheiden: Sanguiniker (Blut), Choleriker (gelbe Galle), Melancholiker (schwarze Galle) und Phlegmatiker (Schleim).

\footnotetext{
${ }^{257}$ Siehe den Teil über die astronomischen Ursachen der Pest, S. 80-81.

258 OCCO/SAYLER/TIEFFEnBACH/TALHAuSER, Was die Pestilentz an jr selbs sey [Augsburg: Philipp Ulhart 1535], S. A III.

${ }^{259}$ Ebd. S. A II.

${ }^{260}$ Zur antiken Viersäftelehre auch als Humorallehre bezeichnet vor allem in: MüLLER, Humoralmedizin; SCHÖNER, Das Viererschema; SIEGEL, Galen's System. Allgemein zur gelehrten Medizin im Mittelater am Beispiel der Pariser Universität im 14. und 15. Jahrhundert: JACQUART, La médecine médiévale.
} 
Wenn nun aber diese ausgeglichene natürliche Mischung der Körperflüssigkeiten wegen einer Störung ins Ungleichgewicht geriete, käme es dadurch zu einer bestimmten Erkrankung, die sich in Funktionsstörungen der Körperorgane äußere. Die Krankheiten werden also als ein widernatürlicher Zustand des Körpers gedeutet, der vornehmlich durch eine verderbliche Veränderung der Körpersäfte verursacht werde. Der erkrankte Körper wäre dann in einer Abwehrreaktion bestrebt, die überflüssigen Säfte und die krankmachenden Stoffe auszustoßen, um dadurch das zerstörte Gleichgewicht wieder herzustellen.

\section{2. Ätiologie der Pest und Vergiftung der Luft}

Für die Erarbeitung des medizinischen Pestkonzeptes im Spätmittelalter und in der Frühen Neuzeit war, wie bereits im vorhergehenden Kapitel erörtert wurde, die antike Medizinlehre essenziell. Zum besseren Verständnis der damaligen Vorstellungen über die Pest ist es zunächst lohnenswert, drei zeitgenössische Definitionen der Seuche aus den Augsburger Pesttraktaten im originalen Wortlaut zu verfolgen. Die vier Stadtärzte in Augsburg stellten die folgende Auslegung der Pestseuche in ihrer Schrift auf: Die Pestilentz ist ain fiebrige/ vergifftel auch befleckende kranckhait und feülung des hertzens/ bluts und gaister so im hertzen seind/ anfengklich und nachfolgend außgetaylt durch die austreybende krefft des hertzens in den gantzen leib. Derhalben ervolgt das dise kranckhait nit allain wesentlich dem blut und gaistern/ sonder auch andern feüchtigkaiten des leibs/ die zu der feülung genaigt/anhanget und zu fellt ${ }^{261}$.

Deutlich ausdrucksvoller wurde die Pest indes von dem Wiener Doktor Johann Engel definiert: Pestilentz (...) kumpt zuzeiten auß dem dampf böser zerstörlicher feüchtigkait/ mit dem element des luffts vermüscht/ daraus dem lufft kumpt ain unschicklichkait oder zerstörlichkait/ woelcher lufft dannn also kumpt zu dem hertzen/zerbricht oder verendert die complex des subtilen bluts der geist und feüchtigkait umb das hertz, die da entzündet werden über die natürlich hitz und vergifft/darnach außgetailt in den gantzen leib des menschen/ darau $\beta$ denn ain pestilentz fieber entspringt. Und alle die menschen die vil solcher böser zerstörlicher feüchtigkait bey in haben/ seind geschickt zu empfahen die pestilentz ${ }^{262}$.

\footnotetext{
${ }^{261}$ OCCO/SAYler/TIEFfenbach/TalHauSER, Was die Pestilentz an jr selbs sey [Augsburg: Philipp Ulhart 1535], S. A III.

${ }^{262}$ ENGEL, Tractat von der Pestilentz [Augsburg: Sigmund Grimm und Marx Wirsung 4. November 1518], S. A II.
} 
Dagegen stellte Doktor Michael Fencken aus Ingolstadt seine klar gefasste Definition der Pest mit der Absicht zusammen, dass diese auch der gmain mann begreifen mag: Die Pestilentz ist/ als die gelerten bezeugen/ ain vergiftung oder feule des luffts/die so sie ain leyb befleckt/vil menschen vergifften mag (...) Die aller maist/ und gemainst ursach diser kranckhait/ ist der faul vergifft lufft/ so durch den oren/ und anderer ort des leibs zum hertzen gezogen wirt/ dardurch das hertz/ als ain fürst aller gelider/ auch des hertzen gaist/ und geblut vergifft/ unnd als dann alle krefft des gantzen leibs mercklich geschwecht/ und zum dickern mal gar benummen werden ${ }^{263}$. Gemäß diesen Darlegungen liegt also das Wesen der Pest in einer giftigen Materie, die in den Körper eindringt und primär das Herz als das zentrale Körperorgan befleckt. Das verursachte eine allgemeine Vergiftung des Blutes und aller Flüssigkeiten um das Herz, die folglich rasch im ganzen Körper Verbreitung fand. Dadurch würde die Zusammensetzung aller körperlichen Säfte gänzlich zerstört und infolgedessen erhöbe sich das sogenannte pestilenzialische Fieber. Je mehr verdorbene Materie in den Körper gelange, desto schneller verliefe die Erkrankung.

An dieser Stelle ist festzuhalten, dass die Autoren der analysierten Pestschriften nicht näher differenzieren, was für ein Stoff genau für die Entstehung der Pest verantwortlich ist. Außer Bezeichnungen wie z.B. böser zerstörlicher feüchtigkait, böse eintruckung oder das gifft der pestilentz wird der Krankheitserreger nicht exakter erfasst, was anschaulich die allgemeinen Erkenntnismöglichkeiten in der vormikrobiologischen Ära widerspiegelt. Die gelehrten Mediziner waren sich dagegen in allen Traktaten einig, dass der menschliche Körper diesen giftartigen Stoff vor allem aus der Luft empfängt. Mit dieser Erklärung ist bereits die prinzipielle Deutung der Pest in der damaligen Medizin angesprochen, die immer auf die Luftvergiftung als wichtigste Ursache hinwies. In der galenisch-hippokratischen Seuchenlehre wurde diese krankheitserregende Materie als Miasma (griech. miásmata) genannt, worunter die Verunreinigungen in der Luft $\mathrm{zu}$ verstehen $\operatorname{sind}^{264}$. Die damalige Deutung der Pestkrankheit wird daher in der Forschung meistens als Miasma-Theorie bezeichnet. Die Mediziner versuchten die pestilenzische Verderbung der Luft zu entschlüsseln und stellten in der Folge verschiedene Erklärungen über den Ursprung dieses Phänomens auf.

\footnotetext{
${ }^{263}$ FENCKEN, Ain gut nützlich Regiment [Augsburg: Alexander Weißenhorn 1533], S. A III-IIIv.

264 LEVEN, Die Geschichte der Infektionskrankheiten, S. 21; DERS., Von Ratten und Menschen, S. 18-19; DINGES, Seuchen in Mittelalter und Früher Neuzeit, S. 20-21; CORBIN, Pesthauch und Blütenduft.
} 


\section{Astrologische Deutungen}

Die Erklärungen über die Einflüsse des Himmels auf den Pestausbruch wurden aus einem philosophischen Grundsatz der Antike abgeleitet, wonach es ist nichts auff disem erdboden/ es hat sein anfang/ und sein ursprung au $\beta$ den hymlen hierab in die welt ${ }^{265}$. Die Sterne und Planeten konnten demnach die ganze Welt und alle Dinge regieren, die aus den vier natürlichen Elementen bestehen. Aus diesem Grund könnten die Bewegungen von Himmelskörpern auch zemynderen und zemeren die vier Elememt/ auch zuverkeren die lüfft und das gewitter/ dardurch sich bewegen kranckhaiten/ und sterbliche zeyt wie dann yetzunder vor augen seind ${ }^{266}$. Für die Entstehung der Pest wären insbesondere bestimmte Konstellationen der Planeten Saturn, Jupiter oder Mars verantwortlich, die bei einem ungünstigen Sternbild das Luftverderbnis auf der Erde hervorrufen könnten. Johannes Engel stellte dazu beispielsweise ganz konkrete Beobachtungen an, die auf den Planet Saturn in Sternzeichen Zwillinge, Waage oder Wassermann als Auslöser der Pestepidemie hinweisen: Darumb rechte ursprüngliche ursach der pestilentz die ich vil und offt erforscht und erfaren hab ist die/ so Saturnus der bö $\beta$ planet ain feind aller lebendiger creatur mit seinem warn lauff kumpt under die zaichen Zwiling, Wag oder Wasserman ${ }^{267}$. Außerdem könnten auch andere astronomische Erscheinungen wie Sonnen- und Mondfinsternis, Kometen oder weitere rätselhafte Himmelszeichen wie beispielsweise flüegendig dracken, brinnende fackel oder springendig gayssen darauf hinweisen, dass in der Zukunft nicht nur Unwetter und Seuchen geschähen, sondern auch Kriege, neue Propheten oder religiöser Aufruhr aufkämen ${ }^{268}$. Astronomische Phänomene wurden im Allgemeinen als warnende Vorboten künftigen Unglücks gedeutet, welche zaichen uns got der allmechtig zu ainer anzaygung und warnung vorhergeschicken wirt/ als durch ain zukünfftige prophezeyung (...) auf das wir uns dester naß mügen bewaren und fürsehen/in allen unseren sachen ${ }^{269}$.

\footnotetext{
${ }^{265}$ KLAINMÜLLER, Ain hymlischen vnn Natürlichen anzaygung [Augsburg: Hans Schönsberger, 12. Oktober 1521], S. A IIv.

266 Ebd.

${ }^{267}$ ENGEL, Tractat von der Pestilentz [Augsburg: Sigmund Grimm und Marx Wirsung 4. November 1518], S. A IIv; Die Bedeutung von Saturn beim Ausbruch der Pestepidemien wurde auch ausführlich behandelt in: SEITZ, Ain schoener Tractat von dem Saturnischen gschoß der Pestilentz [Augsburg: Otmar Silvan, 15. Februar 1522].

268 KLAINMÜLLER, Ain hymlischen vnn Natürlichen anzaygung [Augsburg: Hans Schönsberger, 12. Oktober 1521], S. A III.

${ }^{269}$ Ebd. S. A III-IIIv.
} 
Obwohl die astrologische Deutung der Pest schon in den ältesten Pestschriften, wie z.B. in dem berühmten Pariser Gutachten ${ }^{270}$, formuliert wurde, war diese Theorie unter den Augsburger Doktoren eher umstritten. Vor allem die städtischen Ärzte bestritten eine derartige astrologische Erklärung ${ }^{271}$, die in anderen Traktaten vor allem auf das Werk von Avicenna zurückgeführt werden kann ${ }^{272}$. Die Augsburger Stadtärzte argumentierten, dass die ältesten Mediziner, wie z.B. Galen und Hippokrates oder auch der damals berühmte Doktor Johannes Manardus in Ferrara ausschließlich die Frage der Luft behandelt hätten, ohne sich weiteren Erwägungen über den Einfluss der Sterne darauf zu widmen ${ }^{273}$. Da auch sie sich in ihrer Pestlehre hauptsächlich auf die Werke von Hippokrates und Galen stützen, so die Begründung, legten sie die mögliche Bedeutung der himmlischen Konstellationen ebenfalls nicht näher dar ${ }^{274}$.

\section{Natürliche Ursachen}

Für die Augsburger Stadtärzte waren die natürlichen Ursachen der Luftvergiftung von größter Bedeutung. Darum wiesen sie vor allem auf die Auswirkungen der unregelmäßigen Jahreszeiten hin: die vernünfftigen ursachen fürgeben und die erfarung bezeüget/ und achten/ niemand verstendiger werde zweiflen, das diese Pestilentz erwachse durch unnatürliche zeyt etlicher jar so nach ainander gewesen seind ${ }^{275}$. Der Ausbruch der Epidemie konnte nach der damaligen Auffassung insbesondere dadurch verursacht werden, dass Frühling und Sommer

\footnotetext{
${ }^{270}$ SCHWALB, Gutachten S. 56-61.

271 OCCO/SAYLER/TIEFFENBACH/TALHAuSER, Was die Pestilentz an jr selbs sey [Augsburg: Philipp Ulhart 1535].

${ }^{272}$ Z.B. in ENGEL, Tractat von der Pestilentz [Augsburg: Sigmund Grimm und Marx Wirsung 4. November 1518], S. A II-IIv: und nymm für mich die letst ursach Avicenne/ der da sagt an der obgemelten stat/ das der anfang aller ursach der pestilentz kum au $\beta$ den hymelischen formen/ das seind die bilder der planeten und stern des hymmels.

${ }^{273}$ OCCO/SAYler/TIEFFEnBACH/TALHAuSER, Was die Pestilentz an jr selbs sey [Augsburg: Philipp Ulhart 1535]; Dagegen bezog sich Johannes Klainmüller direkt auf Hippokrates, um seine Ausführungen über die astronomische Deutung zu unterstützen: Es spricht auch unser hochgelerter vatter Hyppocras/ wie das sich die veränderung der lüfft/ alle ding verkeren und veränderen/ besunderlich umb das newe/ und umb den bruch des Mons. In: KLAINMÜLLER, Ain hymlischen vnn Natürlichen anzaygung [Augsburg: Hans Schönsberger, 12. Oktober 1521], S. A IIv-III.

${ }^{274}$ Eine ähnliche Stellungnahme zu dieser Frage auch in BÖHAM, Ain nutzliche/ vnnd für den gemainen Man/ genugsam gegründte [Augsburg: Philipp Ulhart, bearbeitet und ergänzt von Johannes Vogt dem Jüngeren um 1570], S. A III-IIIv: Das aber etlich wollen die ursach den Planeten/ und so de Himmels lauff zu schreiben/ Solchs kann nit mit wenig worten bestätiget/ oder widerfochten werden.

${ }^{275}$ OCCO/SAYLER/TIEFFENBACH/TALHAUSER, Was die Pestilentz an jr selbs sey [Augsburg: Philipp Ulhart 1535], S. A III. Dazu auch eine anschauliche Aussage in JUNG, Ain nutzliche trostliche und kurze underrichtung (1) [Augsburg: Silvan Ottmar 1521], S. A IIv: So nun dise pestilentzichsen leüff/ am fürderlichsten kommen von vergifftung unnd enderung des luffts/ als durch groß enderung der zeit im jar/ so der winter sein natur nit behalt/ nachvolgend der früling/darnach der summer und herbst/ wie dann das jar geschehen ist/ erhaben dann sich böß falin in lüfften/davon die gaist des hertzen bergifft werden/ und solche böse fieber.
} 
warm und feucht waren und danach ein milder und ebenfalls zu feuchter Herbst und Winter folgte. Wenn dann noch im Jahresverlauf keine längere Hitzeperiode stattfände, die die Feuchtigkeit aus der Luft, der Erde sowie den menschlichen Körpern söge, könnten daraus leicht die Fäulnis im Blut und in anderen Körpersäften entspringen. In derartigen Jahren wären vor allem ungekochte Früchte sehr schädlich und es käme zur Verstopfung aller tampf oder schwaißloechlach im Körper, wodurch der mensch genaigt ist solche boese eintruckung zu empfahen ${ }^{276}$. Nach Ansicht der Stadtärzte neigten insbesondere diejenigen Menschen zur Pesterkrankung, die in warmer und feuchter Luft lebten, deren Leib verstopft und unrein sei, die an Müßiggang und Fülligkeit gewohnt seien, wie auch viel Koitus und erschrockenen weybischen herzlosen gemut hätten ${ }^{277}$.

Außer den unzuträglichen Jahreszeiten werden in den Pesttraktaten noch andere Faktoren genannt, die zur Vergiftung der Luft beitrügen, weil, wie die Augsburger Stadtärzte behaupteten, solche schnelle kranckhait auch vil ursach hat ${ }^{278}$. Vor allem waren ihrer Ansicht nach Fäulnisvorgänge aller Art sehr schädlich: Ausdünstungen von Sümpfen und Teichen, stillstehende Gewässer oder unbegrabene Leichen wie auch Abfallstoffe und andere faulende Dinge mit üblem Geruch.

Ferner könne die Luftverderbnis, so die Vorstellung, von einem Erdbeben ausgehen, weil sich dann große Massen von Dampf und verdorbener Luft frei machten. Die schädlichen Fäulnisvorgänge können also aus dem Erdinneren auf das Wasser und die Luft übertragen werden. Auch bei der Öffnung seit langer Zeit verschlossener Brunnen und Gewölbe komme es zur Verunreinigung der Luft. Gelegentlich werden auch Hypothesen aufgestellt, die eine Vergiftung der Luft auf das Erscheinen mysteriöser Kreaturen zurückführen: auch werdent manicherlay seltzamer thier/ auff den bergen und wasseren in die land kummen/dardurch die lüftl vieh und menschen vergyfft werden ${ }^{279}$. Wesen und Aussehen der Tiere werden nicht näher erläutert, ebenso wenig die Frage, wo diese Tiere eigentlich herstammen sollen.

Zusammenfassend lässt sich festhalten, dass in allen Augsburger Pestschriften immer einer oder mehrere der dargestellten Faktoren zur Verunreinigung der Luft genannt wurden. Nach der damaligen Vorstellung verlor die Luft aufgrund dieser verschiedenartigen Vorgänge ihre gesunde Beschaffenheit, weshalb in Folge dessen eine Pestepidemie entspringen konnte.

\footnotetext{
276 OCCO/SAYler/TiefFEnBACh/TalHauser, Was die Pestilentz an jr selbs sey [Augsburg: Philipp Ulhart 1535], S. A IIIv.

${ }^{277}$ Ebd. S. A IV.

${ }^{278}$ Ebd. S. A III.

279 KLAINMÜLLER, Ain hymlischen vnn Natürlichen anzaygung [Augsburg: Hans Schönsberger, 12. Oktober 1521], S. A III.
} 


\section{Ansteckung mit der Pest}

In den Pesttraktaten sind darüber hinaus allgemeine Äußerungen die Übertragung der Pest betreffend enthalten ${ }^{280}$. Für die Ärzte war es offensichtlich, dass die Pest von einem Kranken leicht an einen Gesunden weitergegeben werden konnte. Diese grundsätzliche Erkenntnis wurde beispielsweise schon im ersten gedruckten Gesundheitsregiment anschaulich geäußert: Das haund die hohen maister für ain besonnder ertzney, wann der siechtumb beruerlichen ist und kompt ainen menschen leichticlichen von dem andern an. Man sol sich auch hueten, das man denen nit nach gang, die den presten habent ${ }^{281}$. Dagegen stellte Ambrosius Jung folgende Ermahnung zum Umgang mit den Pestkranken auf, dass am ersten sol soliche person von den gesunden gesondert werden, dann ain mensch mag hundert vergifften ${ }^{282}$. In der zeitgenössischen Wahrnehmung bildeten also alle Kranken einen potentiellen Infektionsherd für sämtliche andere Mitmenschen. An dieser Stelle muss aber die Frage näher erläutert werden, in welcher Art und Weise die Ansteckung mit der Pest von den Medizinern verstanden wurde. Zum Ersten ist zu bemerken, dass in allen analysierten Traktaten bis 1550 die Angaben über die Ansteckungsgefahr nicht in den theoretischen Kapiteln über die Ätiologie der Pest dargelegt werden. Die kurzen Aussagen dazu befinden sich immer nur in den Textabschnitten, welche die praktischen Vorbeugungs- und Heilungsmaßnahmen behandeln. Diese Regelmäßigkeit lässt ohne Frage darauf schließen, dass bis in die Mitte des 16. Jahrhunderts die Infektion an sich nicht als eine der primären Pestursachen definiert wurde. Die Doktoren scheinen in diesem Phänomen weiter nichts als die weiteren Verbreitungsmöglichkeiten der Pest gesehen zu haben, die jedoch initial immer aufgrund der Luftvergiftung ausgelöst wurde. Die Erkenntnis des Infektionsphänomens war also für die Mediziner nur bei der praktischen Pestbewältigung relevant, in den theoretischen Ausführungen schrieb man dieser gar keine Bedeutung zu.

Zum Zweiten ist zu konstatieren, dass von den gelehrten Ärzten um diese Zeit noch keine zusammenhängende Ansteckungslehre ausgearbeitet wurde, mit der sie den Infektionsverlauf zu erläutern versuchten. Erst 1546 stellte der italienische Gelehrte Girolamo Fracastoro (1478-1553) die theoretische Lehre über die Ansteckung (contagio) zusammen und veröffentlichte sie in seiner Druckschrift „De Contagione“ in Venedig. Laut seinen dortigen Ausführungen bestand das Wesen der Ansteckung in spezifischen Krankheitskeimen

\footnotetext{
${ }^{280}$ Die medizinische Erkenntnis der Ansteckungsgefahr findet sich schon in den allerersten Pesttraktaten, darunter vor allem in den Texten von arabischen Gelehrten, vgl. BREHER, Der Memminger Stadtarzt, S. 27-29.

${ }^{281}$ Ordnung der Gesundheit, S. 333.

282 JUNG, Ain nutzliche trostliche und kurze underrichtung (1) [Augsburg: Silvan Ottmar 1521], S. B II.
} 
(seminaria contagionis), die auf drei verschiedene Arten von einem Menschen auf den anderen übergehen und sich dadurch weiter verbreiten können: durch Berührung im direkten Kontakt mit dem Kranken, durch Zunder, d.h. Kleider, Holz und ähnliche Dinge, die geeignet seien, die „seminaria contagionis“ zu bewahren, und auf größere Entfernung durch die Luft ${ }^{283}$. In allen Augsburger Pestschriften bis 1550 sind keine theoretischen Begründungen zu dieser Frage enthalten. Nur aufgrund der in allen Traktaten verstreuten Hinweise kann man annehmen, dass die Ansteckung vor allem im Zusammenhang mit der Vorstellung über die Luftvergiftung gedeutet wurde. In den Ratschlägen für die Aufwärter warnte man ausdrücklich vor der Gefährlichkeit des Atems der Pestkranken, vor dem man sich mit Feueroder Kerzenflammen schützen sollte: Diß gesunden menschen sollend zwischen inen und der siechen haben ain guoten flamen oder für mit durem aichim oder wecholterin oder widin, oder aulbrin holtz, oder habend vast vor inen ain dickfache wechsin brinend kertzen, wan diß für oder flam temperiert, rainget und verzert die vergifftung des luffts und tämpff, das er desterminder dem menschen schädlich mag $\sin ^{284}$. Der vergiftete Atem der Kranken verderbe also gleich den anderen Faktoren die Luft, wodurch die anderen Menschen die giftige Materie in sich aufnehmen könnten. Demgemäß sollte man einen Kranken im Bett entsprechend hoch legen, damit sich sein Kopf höher als die Köpfe der Krankenpfleger oder des Priesters befände: so mag der vergift autum und lufft von dem siechen den priester oder pfleger nit woll treffen $^{285}$.

Außerdem betonte man, dass nicht nur die ausgeatmete Luft, sondern auch alle anderen von dem Kranken ausgehenden Stoffe die vergiftete Materie in sich enthalten könnten. Die Gesunden sollten sich z.B. vor den Tüchern schützen, mit denen man den Kranken ihren Schweiß abwische. Auch die Kleidung und Bettwäsche, die von den Kranken benutzt werde, nehme das Pestgift in sich auf, weil dann ob man schon die farb nit sicht, bleibt doch die krafft in dem hess, als wann man schon den wein au $\beta$ dem fass thut, bleib dannoch der geschmack $^{286}$. Demzufolge könne das im Gewebe angehäufte Gift wieder in die Luft gelangen und somit erneut in den gesunden Menschen eindringen, weil dieser eine derart verdorbene Luft einatmen müsse. Nach der zeitgenössischen Deutung konnte also die Ansteckung sowohl

\footnotetext{
283 Dazu ausführlich in: LEVEN, Die Geschichte der Infektionskrankheiten, S. 36-38; STRASSER, Ansteckungstheorien der Pest, S. 71-73; FOSSEL, Hieronymus Fracastoro; NuTTON, The Reception of Fracastoro's Theory of Contagion.

${ }^{284}$ ELLENBOG, Ordnung, S. 93-94.

${ }^{285}$ Ebd., S. 94.

286 OCCO/SAYLER/TIEFFEnBach/TAlHauser, Was die Pestilentz an jr selbs sey [Augsburg: Philipp Ulhart 1535], S. B Iv.
} 
im direkten Kontakt mit dem Kranken wie auch mit den von ihm gebrauchten Gegenständen aus Tuch erfolgen, die das Pestgift vor allem durch die Luft weiterleiteten.

\section{Verhängnis Gottes als Ursprung der Pest}

In den medizinischen Pesttraktaten wurde der Ausbruch der Pest auch auf eine göttliche Ursache zurückgeführt. Die Mediziner wiesen meist schon in den Einleitungen ihrer Pestschriften darauf hin, dass diese Krankheit wegen der menschlichen Sünden aus einer göttlichen Bestrafung entspränge. Die primäre Ursache einer Pestepidemie läge demnach immer in dem durch Gott herbeigeführten Verhängnis, der mit einer solchen Züchtigung bewirken wolle, dass sich die Menschen durch Buße und Reue von ihrem sündhaften Leben wieder abkehrten. Auch die Luftvergiftung, die damals als die wichtigste natürliche Ursache der Pest galt, wurde letztendlich mit Hilfe des religiösen Diskurses erläutert: ain vergifftung oder feule des luffts (...) on des Allmechtigen verhencknu $\beta$ gar nit entspringen mag; diese kranckhait zu zeyten sey ain straff gottes ${ }^{287}$. Unter allen Augsburger Pestschriften wurde nur in einem einzigen Pesttraktat das Deutungsmuster der direkten göttlichen Strafe in den ätiologischen Erklärungen entschieden abgelehnt. Alexander Seitz (1470-1545) fundierte nämlich in seinem 1522 gedruckten Traktat den Ursprung der damaligen Pestepidemie ausschließlich mit den primären Auswirkungen himmlischer Einflüsse: auß disem allem/ mügt ir gaentzlich guettig glauben das ich nitt unbillicher weiß sprich/das dieser sterbet kumm/ auß art/ und einfluß des himels/ und gar nit auß sonderlicher neüwer ordnung/ oder plag gottes/ und ob wir dannocht dieser straff unwirdig waeren/ so glaub ich dannocht gentzlich/ das dieser sterbent darumb nit underlassen blib $^{288}$. Seinen Erläuterungen folgend entspringe die damals ausgebrochene Pest aus der ungünstigen Konstellation von Saturn und Mars im Sternzeichen Wassermann, die auf alle lebenden Kreaturen ihre zerstörende Wirkungskraft ausstrahlen lasse. Dadurch erhebe sich eine allgemeine Luftvergiftung, die so lange dauern würde, bis im Jahre 1523 Saturn wieder in das Sternzeichen Fische einrücke. Die direkte Intervention Gottes bei der Auslösung der damals ausgebrochenen Pest sei aber auszuschließen, so Seitz, weil die Ordnung der Gestirne und ihr himmlischer Lauf, die anfänglich diese Pestepidemie verursacht hätten, schon bei der Weltentstehung von Gott

\footnotetext{
${ }^{287}$ FenCKEN, Ain gut nützlich Regiment [Augsburg: Alexander Weißenhorn 1533], S. A III.

${ }^{288}$ SeITZ, Ain schoener Tractat von dem Saturnischen gschoß der Pestilentz [Augsburg: Otmar Silvan, 15. Februar 1522], S. 153; und S. 149: Das aber die yetzige pestilentz ain sonderliche straff von got geordnet sey/ ist gantz nit meins beduncken.
} 
erschaffen wurden und seither unverändert blieben. Dieser Deutung nach war die Ursache für den Ausbruch der Pest allein in dem ursprünglich von Gott geschaffenen Lauf der Natur zu suchen und hatte nichts mit den Sünden der Zeitgenossen oder einer Bestrafung derselben durch Gott zu tun. Eine derartige Auslegung, die ein direktes Wirkungsmoment Gottes in Frage stellte und den Ausbruch der Epidemie einzig mit dem Einfluss der Himmelskörper auf die Natur begründete, stellt eine auffallende Ausnahme unter den damaligen Medizinern dar. Die Gründe dafür sind vor allem im ungewöhnlichen Lebenslauf von Alexander Seitz zu suchen, der sowohl in seinen medizinischen Anschauungen wie auch in seiner politischen und religiösen Tätigkeit immer eine radikale Position einnahm ${ }^{289}$. Nach der aktiven Teilnahme am Bauernaufstand 1514 in Württemberg musste er seinen Heimatort Marbach verlassen und sich in die Verbannung in die Schweiz begeben. Anschließend war er von 1519-1521 als Stadtarzt in München tätig, wo er aber wegen der Veröffentlichung seiner provokanten Druckschrift über den Aderlass entlassen wurde ${ }^{290}$. In diesem Traktat setzte er sich nämlich mit dem in München und Bayern herrschenden Missbrauch des Aderlasses bei schwangeren Frauen und mit den dadurch hervorgerufenen Fehlgeburten auseinander und beschuldigte auch die dortige Ärzteschaft sowie die Obrigkeit sich dieses Vergehens schuldig gemacht zu haben. 1522 musste er München wegen seiner radikalen reformatorischen Ansichten verlassen. Nach mehrmaligem Ortwechsel und Verweisungen aus Reutlingen und Zürich schloss er sich in den Jahren 1529-1534 endgültig den Wiedertäufern in Basel an. Sowohl die Laufbahn wie auch das medizinische Schrifttum von Seitz sind keinesfalls üblich für einen Mediziner der damaligen Zeit und bilden vielmehr ein interessantes Beispiel für die medizinische Tätigkeit eines Außenseiters.

Auch wenn Seitz den Ursprung der Pest nur auf astronomische und natürliche Ursachen zurückführen wollte, verzichtete er nicht völlig auf religiöse Deutungsmuster bei der ätiologischen Erklärung der Epidemien. Statt dessen deutet er auch die Möglichkeit der Fürbitte bei Gott an und verweist auf die dadurch eventuell mögliche Abwendung des Unglücks: So mag ich nochmals sprechen/ diser sterbet kumm auß altem lauff und art des himels/ und Saturni/ und gar nit auß sonderlicher neüwer schickung/ oder straff gotes/ ist aber damit nit zu gedencken/ das der sterbet eben muß sein/ und nit müg abgebeten werden $^{291}$. Eine derartige religiöse Auffassung gilt als typisch für die zeitgenössischen

\footnotetext{
${ }^{289} \mathrm{Zu}$ seiner medizinischen und religiösen Tätigkeit in: SCHOTTENLOHER, Doktor Alexander Seitz.

${ }^{290}$ SEITZ, tractat von aderlassen [Landshut: Johann Weyssenburger 1520].

${ }^{291}$ DERS., Ain schoener Tractat von dem Saturnischen gschoß der Pestilentz [Augsburg: Otmar Silvan, 15. Februar 1522], S. 157.
} 
Mediziner, Seitz ausgenommen, die in ihren Traktaten sowohl den Ursprung wie auch das Ende der Pestepidemie letztlich von Gottes Willen abhängig machten.

Die Analyse der Augsburger Pestschriften zeigt deutlich, dass die medizinische Pestlehre bedeutend vom religiösen Diskurs geprägt wurde. Die damalige Medizin war so stark im religiösen Kontext verankert, dass die Entstehung aller Krankheiten und körperlichen Vorgänge ohne ihren Ursprung und ihre Auslösung durch Gott nicht vorstellbar war. Eine alternative Gegenüberstellung von Medizin und Religion als zweier sich ausschließender Deutungsmuster ist im medizinischen Pestschrifttum jener Zeit grundsätzlich nicht $\mathrm{zu}$ erkennen $^{292}$. Abgesehen von den strikt astrologischen Erklärungen von Alexander Seitz wurde die Ätiologie der Pest von den Medizinern allgemein im Zusammenhang mit der göttlichen Wirkungskraft gedeutet. In der theoretischen Auslegung bildete die christliche Religion in den medizinischen Traktaten einen integralen Bestandteil der Pestlehre. Dagegen konnte während der praktischen Pestbewältigung eine zeitliche Verschiebung der religiösen Praktiken erfolgen. Johannes Engel vertrat nämlich in seiner Schrift die Ansicht, dass die medizinische Vorsorge der Annahme der heiligen Sakramente vorausgehen sollte: Am aller ersten sol sich ain mensch got dem allmächtigen befehlen mit rechter rew über sein sünd/ mit solchem fürsatz das er nach der artzney auch genug wöll thun der Christenlichen kirchen/ mit beichten/ und zu empfahen das hochwirdig sacrament ${ }^{293}$. Die Präferenz medizinischer Maßnahmen wurde damit begründet, dass die Arzneien bei der rasch den Kranken befallenden Pest nur bei frühzeitiger Anwendung wirksam wären: die gifft der pestilentz nit zeit gibt (...) und das gifft mag zudringen dem hertzen, dann die artzney von dem hertzen nymmer möchte bringen $^{294}$. Im Traktat von Engel, welches innerhalb des Augsburger Pestschrifttums in dieser Hinsicht eine Ausnahme darstellt, bleibt der religiöse Diskurs in seinen Grundlagen dennoch weiter unangefochten.

\section{Heiligenverehrung in der Reformationszeit im Spiegel der Augsburger Pestschriften}

Die Zeitgenossen im Spätmittelalter wandten sich mit ihren Gebeten an Gott, um die schnellere Abwendung der Pest zu erbitten. Während der Ausübung dieser religiösen Praktiken wurden insbesondere die Pestheiligen Sebastian und Rochus wie auch die

\footnotetext{
${ }^{292}$ Vgl. auch zum Verhältnis der Medizin und Religion in der Pestlehre in: LANG, Erklärung, S. 153-158; ESSER, Pest, S. 56-59.

${ }^{293}$ ENGEL, Tractat von der Pestilentz [Augsburg: Sigmund Grimm und Marx Wirsung 4. November 1518], S. C Iv. ${ }^{294}$ Ebd.
} 
Gottesmutter Maria um ihre spezielle Fürsprache bei Gottvater angerufen. Die wichtige Rolle des Heiligenkultes bestätigen vor allem die seit den Sechziger Jahren des 15. Jahrhunderts in Augsburg gedruckten religiösen Pestblättern ${ }^{295}$. Aus der ersten Hälfte des 16. Jahrhunderts sind dagegen in Augsburg keine religiösen Pestblätter überliefert, die sich explizit auf die Heiligenverehrung in der Pestzeit beziehen. Die große Bedeutung von Pestheiligen in der Zeit kurz vor den reformatorischen Umbrüchen in Augsburg zeigt aber beispielsweise eine kurze gedruckte Auslegung des Gebets „Pater Noster“ für die Pest-, Kriegs- und Hungerszeit, dessen Titelseite mit einer Darstellung Gottvaters mit dem Schwert auf einer Wolke sitzend und darunter Heiligen Sebastian und Rochus bebildert wurde ${ }^{296}$. Ausdrückliche Empfehlungen für Gebete an die Heiligen Sebastian und Rochus befinden sich auch in den medizinischen Inkunabeln ${ }^{297}$. Im Zusammenhang mit der Heiligenverehrung lassen sich jedoch bereits in der ersten Hälfte des 16. Jahrhunderts die reformatorischen Einflüsse in den medizinischen Pestschriften in Augsburg deutlich erkennen.

Obwohl die Reformation in Augsburg endgültig erst am 17. Januar 1537 mit der völligen Verbannung „der papistischen Abgötterei“ und der Übergabe aller katholischen Kirchen an die Protestanten vom großen Rat eingeführt wurde, befanden sich schon in den ersten Jahren nach dem Auftritt Luthers viele Anhänger der Erneuerung des Christentums unter der Augsburger Bevölkerung ${ }^{298}$. Neben den Lutheranern waren aber auch die Zwinglianer besonders stark in der Stadt vertreten, denen sich im Laufe der Zwanziger Jahre die Mehrheit der Neugläubigen angeschlossen hatte. Unter den Ratsherren gewann die evangelische Partei hingegen erst mit Beginn der Dreißiger Jahre eine deutliche Oberhand. Vor diesem Hintergrund sind die seit 1533 energisch erlassenen Verordnungen des Rates zur Unterdrückung der katholischen Religion zu sehen, wie z.B. das noch im selben Jahr erlassene Verbot aller Umgänge, Wallfahrten und Prozessionen in der Stadt ${ }^{299}$. Die von den Protestanten 1537 in Augsburg erreichte Vormachtstellung dauerte aber nur bis zum Jahre 1547 an, nachdem der von Augsburg unterstützte Schmalkaldische Bund eine Niederlage erlitten hatte. Infolge dessen wurde die Stadt von Kaiser Karl V. gezwungen, die erneute

\footnotetext{
${ }^{295}$ Siehe, S. 46-57.

${ }^{296}$ ANONYMUS, Ain Pater noster zu beten [Augsburg: Sigmund Grimm und Marx Wirsung 1521].

297 TORMANITA, Vil menschen weren der pestilentz frey (1-2) [Einbl. Augsburg: Günther Zainer um 1472]; JUNG, Ein außerwelt loblich tractat [Augsburg: Hans Schönsberger, 14. November 1494]; auch in dem ersten gedruckten deutschsprachigen Pesttraktat: STEINHÖWEL, Büchlin der ordnung [Ulm: Johannes Zainer von Reutlingen, 11. Januar 1473].

${ }^{298}$ Zur Reformation in Augsburg vor allem: IMMENKRÖTER, Kirche; ROTH, Reformationsgeschichte, WARMBRUNN, Konfessionen.

${ }^{299}$ Am 22. Juli 1534 wurde die katholische Predigt in der Stadt verboten und die Gottesdienste von Katholiken auf jene acht Kirchen beschränkt, die dem Bischof oder dem Domkapitel unterstanden, in: IMMENKRÖTER, Kirche, S. 398.
} 
Zulassung des katholischen Ritus und die Rückgabe der meisten Kirchen an die Katholiken hinzunehmen. Zur Durchsetzung des für eine Übergangszeit die kirchlichen Verhältnisse regelnden Interims und zum wirksamen Schutz der katholischen Geistlichkeit in Augsburg griff der Kaiser 1548 auch in die städtische Verfassung ein und beseitigte das alte Zunftregiment. An dessen Stelle wurde ein neues Stadtregiment mit rechtlicher Parität von katholischer und lutherischer Konfession eingeführt, die de facto eine dauerhafte Bikonfessionalität in Augsburg begründete. Mit dem Augsburger Religionsfrieden von 1555 wurde schließlich die Auflösung der religiösen Einheitlichkeit in der Stadt zugunsten der Pluralität und der sich neu herausbildenden Bekenntnisse endgültig besiegelt ${ }^{300}$.

Schon für die frühe Reformationszeit sind einige reformatorische Anhänger unter der Augsburger Ärzteschaft belegt. Die Brüder Jung, Ambrosius (1471-1548) und Ulrich (14781539), beide promovierte Mediziner und Augsburger Stadtärzte, traten offenbar schon im Jahre 1518 während des Aufenthaltes Martin Luthers in Augsburg auf die Seite der Reformation $^{301}$. In der Mitte der Zwanziger Jahre schlossen sie sich aber den Zwinglianern an, deren eifriger Anhänger auch ein anderer Augsburger Stadtarzt, Gereon Sailer (gest. 1562), war $^{302}$. In den Dreißiger Jahren nahmen die Doktoren aktiv an der Regelung des Kirchenwesens in Augsburg teil. Einer von ihnen, Ambrosius Jung, war als weltlicher Kirchenpropst tätig und beriet gemeinsam mit den anderen Pröpsten und den Predigern die kirchlichen Angelegenheiten der $\mathrm{Stadt}^{303}$. Darüber hinaus waren noch mindestens zwei Augsburger Stadtärzte nachweisbar der evangelischen Konfession beigetreten: Adolph Occo II. (1494-1572) $)^{304}$ und Sigmund Grimm, der als Drucker reformatorischer Flugschriften Anfang der Zwanziger Jahre tätig war ${ }^{305}$.

In diesem Zusammenhang scheint es offensichtlich eine direkte Auswirkung der Reformation zu sein, dass sich unter den in der ersten Hälfte des 16. Jahrhunderts in Augsburg gedruckten Pesttraktaten nur in zwei Texten von auswärtigen Autoren eine direkte Bezugnahme auf die Pestheiligen finden lässt. In der Pestschrift eines anonymen Autors aus Zürich werden schon in der Einleitung drei umfangreiche Gebete zu Maria, zum Heiligen Sebastian und zum

\footnotetext{
300 WarmbrunN, Konfessionen, S. 69-88, 98-114, 121-130; IMMENKRÖTER, Kirche, S. 401-403; ROTH, Reformationsgeschichte IV, S. 170-199. Die für das neue Stadtregiment festgelegte Parität von Katholiken und Protestanten der „Confessio Augustana“, die mit dem Augsburger Religionsfrieden von 1555 erneut bestätigt wurde, zwang nunmehr in den Fünfziger Jahren des 16. Jahrhunderts die evangelische Partei zur Abkehr von Zwingli und zur Anlehnung an das Luthertum.

${ }^{301}$ FLEISCHMANN, Ärztefamilie, S. 25-29.

${ }^{302}$ Zum Leben von Gereon Sailer in: MARTZ, Gesundheitswesen, S. 25-26.

${ }^{303}$ FLEISCHMANN, Ärztefamilie, S. 27-29.

${ }^{304}$ BÜHLER, Ärztegeschlecht.

${ }^{305}$ IMMENKRÖTER, Kirche, S. 394. Ein kurzer Lebenslauf von Grimm in MARTZ, Gesundheitswesen, S. 21.
} 
Heiligen Rochus dargereicht, die in der Pestzeit vorgelesen werden sollten ${ }^{306}$. Dazu wurde der Gebettext jeweils mit einem Bild des entsprechenden heiligen Fürsprechers ergänzt. In der Pestschrift von Doktor Michael Fencken aus Ingolstadt heißt es, dass man alle Sünde und Missetaten ehrlich bereuen, beichten und büßen und sich an Gott und seine Heiligen wenden solle, darunter sonderlich den ritterlichen Patronen S. Sebastian/ täglichs bitten und anruffen $^{307}$.

In den Pestschriften der Augsburger Autoren aus der ersten Hälfte des 16. Jahrhunderts finden sich hingegen keine Empfehlungen für die Verehrung spezieller Heiliger in der Pestzeit. Als der einzige Vermittler zu Gottvater wurde nun Christus genannt, an den man seine Bittgebete zuerst richten solle: Derhalb unser radt/ das man sich anfangs Got dem Allmaechtigen gantz und gar ergeb durch Jesum Christum unsern hayland/ mit gantzem hertzlichem layd vergangner und aller sünd halben/ auch mit ernstlichem anrueffen umb gnad und hilff götlichen willen zu gedulden ${ }^{308}$. Auch wenn man aus dem Fehlen direkter Bezüge auf die Pestheiligen noch keine endgültigen Schlussfolgerungen ziehen kann, geben einige weitere Hinweise Aufschluss darüber, dass in den Augsburger Pestschriften spätestens seit den Dreißiger Jahren des 16. Jahrhunderts eine strikt protestantische Stellung bezüglich der Ablehnung der Heiligenverehrung eingenommen wurde. In zwei Ausgaben eines Einblattdruckes aus der Zeit um 1472 (Abb. 8-9) wurde ein populärmedizinisches Gedicht für die Pestzeit noch um ein Bild mit dem Martyrium des Heiligen Sebastian ergänzt ${ }^{309}$. In dieser kurzen Unterweisung wurde direkt dazu aufgerufen, die eigenen Gebete speziell an den Heiligen Sebastian zu richten:

Des ersten halt den rat den ich meyn

Wann das duncket mich sicher nit kleyn

Das man in dieser sach ernstlich sol

Anruffen got das hilffet sicher wol

Sant sebastians auch nich vergi $\beta$

Wann sein helffen ist auch gar gewiß.

In einer erneuten Ausgabe dieses medizinischen Einblattdruckes aus dem Jahre 1536 wurden allein die zwei letzten Zeilen des zitierten Abschnittes ausgelassen ${ }^{310}$. Auch die Darstellung des Martyriums des Heiligen Sebastians wurde durch eine ornamentale Umrahmung ersetzt

\footnotetext{
${ }^{306}$ ANONYMUS, Ain regiment wider Die Pestilentz, [Augsburg: Johannes Schönsberger um 1525].

${ }^{307}$ FENCKEN, Ain gut nützlich Regiment [Augsburg: Alexander Weißenhorn 1533], S. A IIIv.

308 OCCO/SAYLER/TIEFFENBACH/TALHAUSER, Was die Pestilentz an jr selbs sey [Augsburg: Philipp Ulhart 1535], S. D I-Iv.

${ }^{309}$ TORMANITA, Vil menschen weren der pestilentz frey (1-2) [Einbl. Augsburg: Günther Zainer um 1472].

${ }^{310}$ ARDEA, Ain gut und vast nutzliches Regiment [Einbl. Augsburg: 1536].
} 
(Abb. 12). Der Zeitpunkt der Neuauflage dieses medizinischen Pestdruckes fällt in die Mitte der reformatorischen Umwandlung in Augsburg. Es ist daher zu vermuten, dass die Streichungen eine unmittelbare Folge der sich zu diesem Zeitpunkt durchsetzenden Reformationsbestrebungen waren.

Einen anschaulichen Beleg für die Ablehnung der Pestheiligen unter den Augsburger Protestanten liefern auch zwei Versionen eines anderen Druckbildes ${ }^{311}$. Im Jahre 1530 erschien in einem spanischen Gesundheitsbuch von Luis Lobera de Avila ein Holzschnitt, welcher die verheerenden Auswirkungen der Pest darstellte ${ }^{312}$. Im Vordergrund des Bildes liegt ein toter Mann auf dem Boden, der von verschiedenen Tierkadavern umgeben ist. Darunter sind mehrere Vögel sowie ein Hund, eine Katze und ein Hahn (Abb. 13). Darüber befindet sich ein Pestkranker im Sterbebett, der von zwei sich die Gesichter mit Tüchern schützenden Personen - ein Mann und eine Frau - begleitet wird. Links im Hintergrund des Bildes sind über einem verstorbenen Kind und dem Kadaver eines Pferdes auch die typischen Darstellungen der Pestheiligen Sebastian und Rochus zu sehen. Der gleiche Holzschnitt erschien erneut im Jahr 1539 in einer deutschen Übersetzung von „De remediis utriusque fortunae“, einer moralphilosophischen Trostschrift des italienischen Humanisten Francesco Petrarca (1304-1374) $)^{313}$. Das Bild wurde in diesem Zusammenhang von dem Augsburger Drucker Heinrich Steiner als Titelillustration im Kapitel Von dem Sterben oder Pestilentz verwendet, aber mit einem beachtlichen Unterschied. Für das protestantische Publikum in Augsburg waren nach der endgültigen Durchsetzung der Reformation die Darstellungen von den Pestheiligen Sebastian und Rochus völlig getilgt worden und an ihrer Stelle war einfach ein unbedrucktes weißes Feld gelassen worden (Abb. 14).

\footnotetext{
${ }^{311}$ Mauelshagen, Pestepidemien, S. 248-251.

${ }^{312}$ LOBERA DE AVILA, Vanquete de nobles cavalleros [Augsburg: Heinrich Steiner 1530].

${ }^{313}$ Petrarca/Vigilius (ÜBers.), Das Glückbuch/ Beydes des Gutten und Boesen [Augsburg: Steiner Heinrich 1539].
} 


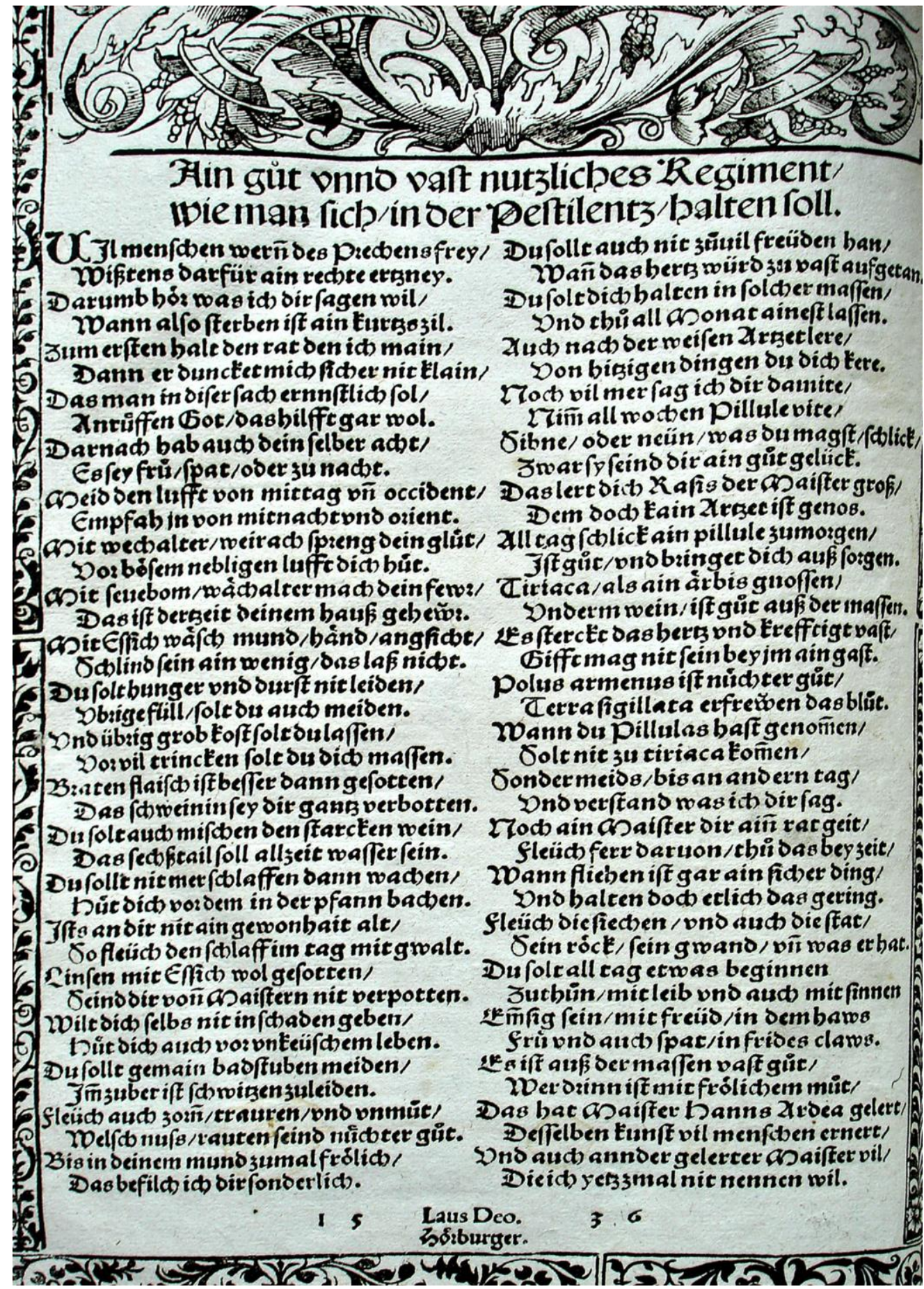

Abb. 12: ARDEA, Ain gut und vast nutzliches Regiment [Einbl. Augsburg: 1536] 


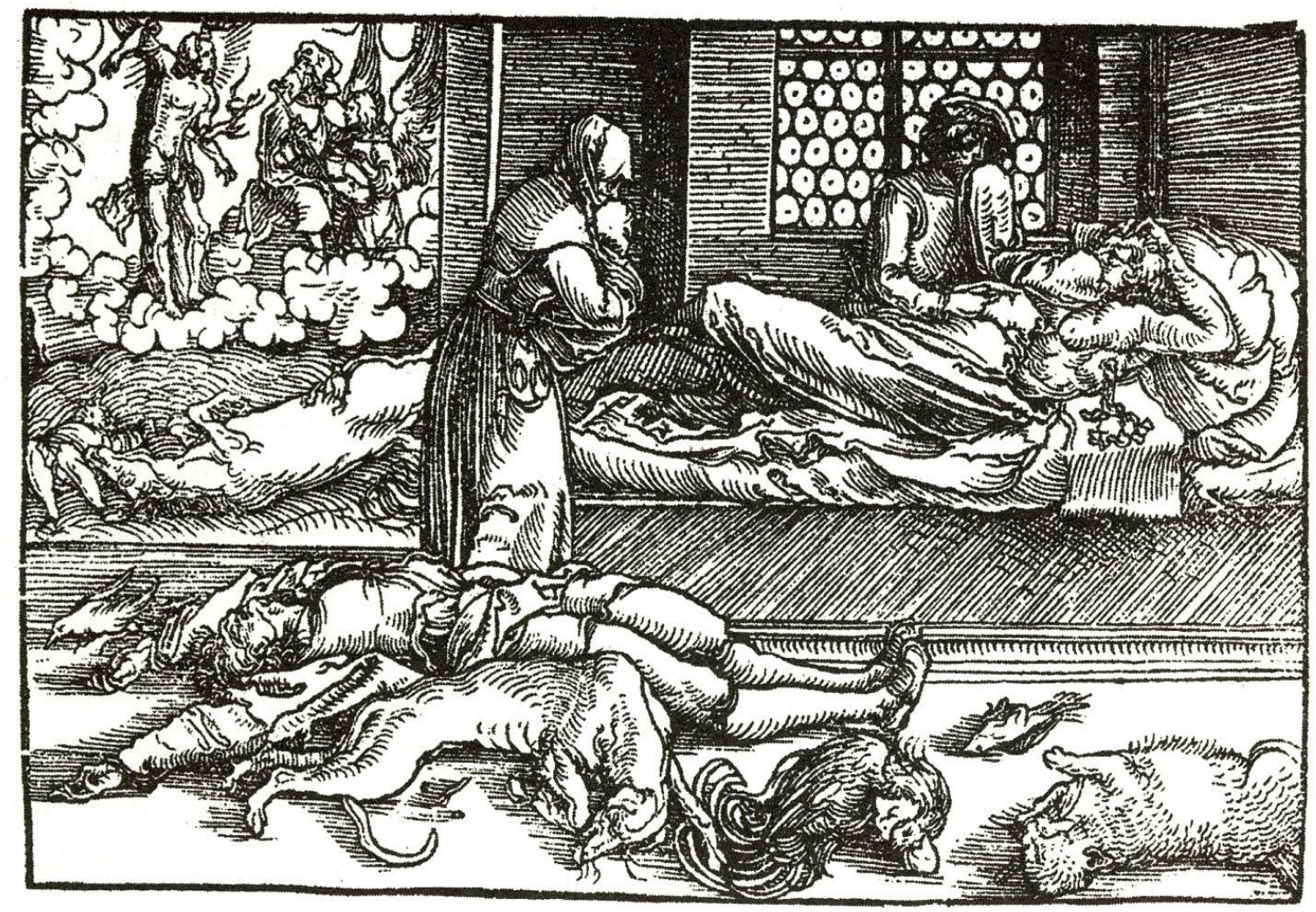

Abb. 13: Holzschnitt über die Auswirkungen der Pest in: LOBERA DE AvILA, Vanquete de nobles cavalleros...[Augsburg 1530]

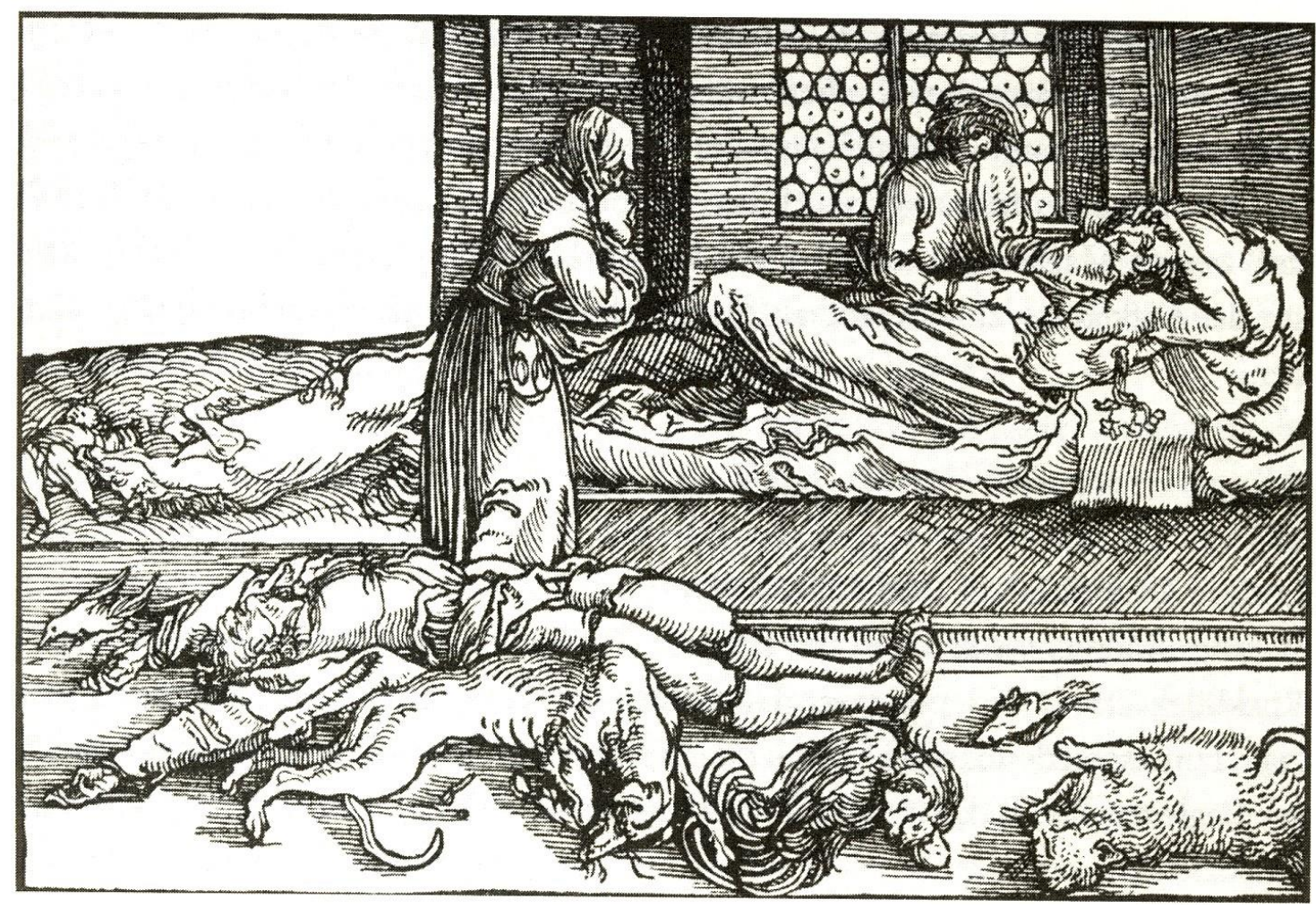

Abb. 14: Holzschnitt über die Auswirkungen der Pest in: PETRARCA, Das Glückbuch/ Beydes des Gutten und Boesen [Augsburg 1539] 
Auch anhand der Pestschriften von Ambrosius Jung, der sich von Anfang an der Reformationsbewegung mit voller Eifer anschloss, sind die sich vollziehenden Veränderungen in der Einstellung zur Heiligenverehrung zu verfolgen. In seinem ersten Pesttraktat aus dem Jahre 1494, der im Auftrag des Augsburger Domkapitels zusammengestellt wurde, nimmt die Versorgung der Seele während der Pestzeit einen wichtigen Platz ein ${ }^{314}$. Dazu wurde vor allem die andachtsvolle Anrufung Marias und der Heiligen Sebastian und Rochus empfohlen. Dagegen verzichtete er in der Pestschrift aus dem Jahr 1521 bereits vollständig darauf, die Kranken an die Hilfeersuchung bei den Pestheiligen $\mathrm{zu}$ gemahnen, worin sich vermutlich sein frühes Interesse für die Reformation widerspiegelt $^{315}$. Auch seine direkte Bezugnahme auf das Matthäusevangelium, die in anderen Pestschriften zu dieser Zeit keineswegs üblich war, bestätigen diese Annahme ${ }^{316}$. In der nächsten Ausgabe seines Werkes aus dem Jahr 1535 $5^{317}$, als Ambrosius Jung schon seit längerer Zeit als aktiver Anhänger der zwinglianischen Reformationsrichtung tätig war, wurden die religiösen Ausführungen über die Pest eindeutig im protestantischen Sinne umgearbeitet $^{318}$. In der umfangreichen Einleitung erklärt Jung anhand der Auslegung zahlreicher Bibelstellen vornehmlich die Frage, warum die Menschen von Gott mit der Pest bestraft würden ${ }^{319}$. Zunächst stellt er die Menschenwerdung und Opfergabe von Christus dar, der die Menschheit von der Erbsünde erlöse. Da aber die Menschen wegen ihrer sündhaften Natur ständig in Sünde und Laster fielen, züchtigt Gott die Menschheit mit vielerlei gerechten Strafen wie z.B. Krankheit, Teuerung und Krieg. Weil aber solche Züchtigung bisher keine beachtliche Besserung des menschlichen Verhaltens bewirkt habe, schickt er unns yetzunder zum letsten die schwaere und erschrockliche kranckhait/ die doch zu dieser zeit wenig natürlich ursach hat// die Pestilentz/ die dann also forchtsam den menschen ist/das ains aller liebe/trew und freundtschaft vergessen ist/ und von ainander fliehen in aller not/ wie dann vor augen $^{320}$. Diese schwere Strafe sei von Gott nur deswegen verhängt worden, das wir von

\footnotetext{
314 JUNG, Ein außerwelt loblich tractat [Augsburg: Hans Schönsberger, 14. November 1494].

${ }^{315}$ DERS., Ain nutzliche trostliche und kurze underrichtung (1) [Augsburg: Silvan Ottmar 1521].

${ }^{316}$ Ebd. S. A II-IIv: durch sant Matheum sprechend/ kommend alle her zu mir die in diesem sterblichen blöden leyb arbaiten/ in triebsal/ widerwerigkait/ kranckhait/forchten/ und damit beladen seyt/ ich bin der artzt der euch erneren und gesund machen will/ an eüweren mangeln/Die ertzney meines jochs ist süß/ und mein bürden ring.

${ }^{317}$ DERS., Ain nutzliche trostliche und kurze underrichtung (2) [Augsburg: Silvan Ottmar 1535]; Mit derselben religiösen Einleitung auch die nächsten Textauflagen 1539 und 1563.

${ }^{318}$ Auf die protestantische Ausprägung der religiösen Erörterungen in dieser Pestschrift wies schon Karl Sudhoff hin, in: SUDHOFF, Inkunabeln, S. 182.

${ }^{319}$ In seinen religiösen Erklärungen beruft er sich vor allem auf die folgenden Bücher des Neuen Testaments: Matthäus-Evangelium, Paulusbriefe: 1. Brief an Timotheus, Brief an die Römer, Brief an die Hebräer, 1. Brief an die Korinther, und 1. Johannesbrief. Aus dem Alten Testament werden dagegen das Buch der Weisheit und das Buch Kohelet, 38 zitiert.

${ }^{320}$ JUNG, Ain nutzliche trostliche und kurze underrichtung (2) [Augsburg: Silvan Ottmar 1535], S. A IIv.
} 
unnsern sünden abstanden/ zum Herrn fliehen in ainem waren glauben/liebe und hoffnung unser leben bessern (...) darzu uns seine hailigen Evangelia ermanen ${ }^{321}$. Der Rückkehr zu Gott könne dabei nur durch die Vermittlung von Christus geschehen, der nach protestantischer Auslegung als einziger Fürsprecher fungiere, der die göttliche Peststrafe, wie zuvor die Erbsünde wirksam abwenden könne: wollen wir anderst dem wol verdienten zorn Gottes empfliehen/ so in solichen schwaeren leüffen yetzunder uns zu stat/ und auß gnaden Gottes sein trost haben/müssen wir mit ainem rechten glauben/der durch die liebe wircket/ und on zweyfleter hoffnung zu Christo Jesu unserm hayland fleiehen/ von im bitten und begeren/ das er sollichs bey seinem hymlischen vater unserm Got/ auß gnaden und barmherzigkait wolle abwenden ${ }^{322}$.

In der protestantischen Auslegung des Fürsprechens während der Pestzeit wurden die Gottesmutter Maria und die üblichen Pestheiligen wie Sebastian oder Rochus völlig abgeschafft und die Bedeutung Christi in den Vordergrund der religiösen Pestbewältigung gerückt.

\footnotetext{
${ }^{321}$ Ebd.

${ }^{322}$ Ebd. S. A III; Christus als einziger Fürsprecher gegen die Pest wird in den protestantischen Pestgebeten auch für den Zeitpunkt des Todes ausdrücklich hervorgehoben in: THEOPHILUS, Zwu Gotselige/ Gnadenreiche vnd Christliche/ Bekantnussen unsers heyligen Christlichen Glaubens [Augsburg: Heinrich Steiner 1544].
} 


\section{Medizinische Anweisungen in den Pestschriften}

In allen Traktaten nimmt die geeignete Prophylaxe zum Schutz vor der Pest einen gewichtigen Platz ein. Grundsätzlich handelt es sich hier um verschiedenartige Anweisungen, denen zufolge die korrekte Beachtung der Maßnahmen vor der individuellen Erkrankung und der weiteren Ausbreitung der Pestepidemie schützen könne. Die verordneten Vorbeugemaßnahmen basierten dabei auf den zeitgenössischen Anschauungen über die Ätiologie der Pest und stellen de facto ihre praktische Umsetzung im Alltag dar. Das Grundziel solcher Maßnahmen war es insbesondere, eine gesunde Vermischung aller Säfte im Leib beizubehalten und alle schädlichen Faktoren zu vermeiden oder zumindest zu beschränken. In dieser Hinsicht sind in den Pesttraktaten zwei grundsätzliche Richtlinien zu erkennen. Zunächst werden bestimmte Maßregeln für die Pestzeit aufgestellt, die jeder selbst in seiner Diätetik, Wohnungs- und Lebensweise beachten solle. Daneben solle man verschiedene Prophylaktika zum täglichen Gebrauch einnehmen und eine individuelle Reinigung des Körpers von überschüssigen Flüssigkeiten nach der Beratung von Medizinern durchführen.

Die medizinischen Anweisungen wurden immer aufgrund der sogenannten sex res non naturales zusammengestellt, die die Grundlage aller Prophylaxemaßnahmen in der damaligen Medizinlehre bildeten ${ }^{323}$. Mit den sechs „unnatürlichen Dingen“ sind solche Krankheitsfaktoren gemeint, die nicht in der Natur des menschlichen Leibs lägen, sondern von außen auf die körperliche Konstitution einwirken könnten. Dazu gehören verschiedenartige Faktoren der Umwelt, die meistens in folgende sechs Kategorien eingeordnet wurden: 1. Luft, 2. Speise und Trank, 3. Schlaf und Wachen, 4. Ruhe und Bewegung, 5. Ausscheidung und Zurückhaltung von Substanzen, 6. Gemütserregungen ${ }^{324}$. In allen Pestschriften bleibt zwar der theoretische Ausgangspunkt der Prophylaxe immer gleich, aber in ihrer detaillierten Ausrichtung sind viele Unterschiede zu vermerken. Zumeist werden nur einige bestimmte vorbeugende Maßnahmen angegeben, die vom Autor eines Pesttraktates aufgrund seiner eigenen Erfahrungen aus der Pestzeit als besonders empfehlenswert erachtet wurden. Deswegen sind gelegentlich auch spezielle Anleitungen anzutreffen, die Vorsorgenaßnahmen nur für einen spezifischen Lebensbereich als notwendig erachteten und darauf hinwiesen.

\footnotetext{
${ }^{323}$ Vgl. MÜLLER, Humoralmedizin, S. 82-87.

${ }^{324}$ Genau nach dieser Einordnung wurden die Prophylaxemaßnahmen in dem Pesttraktat von vier Augsburger Stadtärzten zusammengestellt, OCCO/SAYLER/TiEFFENBACH/TALHAUSER, Was die Pestilentz an jr selbs sey [Augsburg: Philipp Ulhart 1535].
} 


\section{Schutz vor der verdorbenen Luft}

Die meisten Belehrungen von Medizinern betrafen die schädlichen Auswirkungen der Luft, was sich logischerweise aus der damaligen Ätiologie der Pest herleitete. Grundsätzlich galt hier die allgemeine Regel, dass trueber/ taempfiger/ nübliger/ und feüchter lufft/ ist dise zeyt schedlich $^{325}$. Auf derartige Luft müssten insbesondere die Menschen achten, die inn tieffen wohnungen/ tuncken und der geleichen orten wohnten ${ }^{326}$. Der Südwind sei dabei wegen seiner Wärme und Feuchtigkeit besonders gefährlich, weshalb man die Hausfenster generell nur bei kälterem Nordwind aufmachen solle. Auch besitze vornehmlich die Luft, welche vom Mittag bis Mitternacht wehe, eine ungesunde Beschaffenheit. Während dieser Zeit müssten deshalb alle Gemächer verschlossen bleiben. Indessen sei die Luft ab bzw. nach Mitternacht und insbesondere die bei Sonnenaufgang zuträglicher, weil sie dann natürlicherweise von ihren schädlichen Eigenschaften gereinigt werde. Wenn sie jedoch zu feucht und warm sei, solle man sich auch vor dieser hüten. Zum Schutz vor der verdorbenen Luft empfahlen die Doktoren den Gebrauch verschiedener Präventivmittel. Bevor man aus dem Haus gehe, sei es nützlich, sich den Mund mit Wein und Rosenwasser auszuwaschen oder die aromatischen Dünste aus im Wasser getränkten Kräutern wie z.B. Raute und Lorbeer einzuatmen. Nach dem Verlassen des Hauses sollen die Menschen permanent Zitterwurzel, Weißwurz, Muskatnuss oder Nelken im Mund halten, weil die ding rechtfertigen den lufft so du an dich zeüchst/ unnd nehmen ime sein übrige feüchte ${ }^{327}$. Für die Reichen empfahl man die teureren Zitronenkörner, dagegen sollten die Armen zu diesem Zweck einfach Lorbeerblätter gebrauchen.

Darüber hinaus sollen die Menschen weitere wohlriechende Dinge an ihrem Körper tragen. Diesem Zweck dienen vor allem apfelförmige Kugeln oder Büchsen, die mit aromatischen Stoffen wie etwa Lavendel, Rosen, Veilchen, Zimtrinde, Muskatnuss, Nelken, Weinrauten, Amber oder Rosmarin gefüllt waren. Solche Duftkompositionen konnten nach den beiliegenden Rezepten selbst zubereitet oder direkt beim Apotheker erworben werden. Dazu empfahl man unterstützend ein mit einem Kräuterauszug oder Duftwasser getränkten Schwamm bzw. ein damit benetztes Tuch. Dadurch erhoffte man, sich vor der schlechten Luft wirksamer schützen und auch das Herz zusätzlich kräftigen zu können.

\footnotetext{
325 Occo/SAYler/TiefFenbach/TalHauSER, Was die Pestilentz an jr selbs sey [Augsburg: Philipp Ulhart 1535], S. B I.

326 Ebd.

327 Ebd. S. B II.
} 
Neben der Luftverderbnis warnten die Ärzte ausdrücklich vor der weiteren Vergiftung der Luft durch allerlei Gestank, weil dies eine entscheidende Ursache für eine leichtere Anfälligkeit für die Pest sei: das kain ding sey das mer feind sey der natürlichen hitz des hertzens dann böser stinckender geschmack/ der da verendert und zerstört das subtil blut des lebendigen gaists $^{328}$. Aufgrund dieser allgemeinen Wahrnehmung wurden in den Pestschriften verschiedene Hygienemaßnahmen vorgeschlagen. Zunächst sei dafür zu sorgen, dass man saubere Kleidung trage, da schmutzige Wäsche zur allgemeinen Vergiftung eines Menschen beitragen könne: Alle klaidung sol sauber und one gestanck sein/ darmit ime selbs der mensch kain ursach sey des vergifftens ${ }^{329}$. Die Autoren aller Traktate betrachteten darüber hinaus die gründliche Reinhaltung von Häusern und Gassen als oberstes Hygienegebot: man sol auch die heüser sauber und rain halten/ des gleichen die rynnen/ und haimliche gmach/ auch die gassen vor allen übelriechenden dingen ${ }^{330}$. In diesem Zusammenhang sei insbesondere auf die richtige Hygiene in den sogenannten „heimlichen Gemächern“ zu achten, in denen die „Kammerstühle“ standen und auch die Nachttöpfe abgestellt wurden. Zur Reinigung der Luft in diesen Kammern wie auch in allen anderen Wohnräumen sei vor allem Rauchwerk zu gebrauchen, mit dem die üblen Ausdünstungen der Luft beseitigt werden können. Zu diesem Zweck sei das Verbrennen von wohlriechenden Hölzern wie Wacholder, Zypresse, Eiche, Tanne oder Weide von großem Nutzen. Als ebenso nützlich galt es, ein kleines Feuer auf dem Hof vor den Häusern zu entzünden.

Für die wirksame Durchführung der Räucherungen in den Wohnräumen empfahl man die von den Apothekern angebotenen Kerzen und Rauchkugeln. Diese wurden aus Duftstoffen wie z.B. Lorbeer, Weihrauch, Rosmarin, Myrre, Nelke, Thymian, Salbei, Muskat, Aloe oder Kampfer hergestellt. Solche kostbaren Räucherwerke konnten sich jedoch in aller Regel nur vermögende Menschen leisten. Aus diesem Grund boten die Ärzte auch einige Methoden speziell für Arme an. Diese können z.B. in ihren Wohnstuben einfach die Wacholderbeeren oder andere pulversierte Kräuter auf die Glut legen. Die Räucherwerke in den Häusern sollen dabei vor allem während der Winterzeit angewendet werden. Für denselben Effekt solle man im Sommer duftende Blumen und Kräuter verstreuen oder die Wohnräume mit Rosen-, Lavendel-, Rauten-, oder Nelkenwasser unter Zusatz von Essig besprengen. Durch

\footnotetext{
${ }^{328}$ ENGEL, Tractat von der Pestilentz [Augsburg: Sigmund Grimm und Marx Wirsung 4. November 1518], S. A IV.

${ }^{329}$ BÖHAM, Ain nutzliche/ vnnd für den gemainen Man/ genugsam gegründte vnderricht [Augsburg: gedruckt von Philipp Ulhart und zum Neudruck aufgelegt von Johannes Vogt dem Jüngeren um 1570; bearbeitet und ergänzt von Wolfgang Talhauser um 1547], S. B II-IIv.

${ }^{330}$ KLAINMÜLLER, Ain hymlischen vnn Natürlichen anzaygung [Augsburg: Hans Schönsberger, 12. Oktober 1521], S. C IIIv.
} 
wechselnde Anwendung verschiedener Duftstoffe glaubte man eine wirksamere Reinigung der Luft erreichen zu können.

Zum Schutz vor der vergifteten Luft solle man alle Stätten meiden, an denen sich schlechte Gerüche erhöben: man sol fliehen allen stinckenden übelschmeckenden lufft/ der bey haimlichen gemaechen/ oder bey Moetzgen und andern wincklen ist/ dahin der gemain hauff sein überflu $\beta$ und unrainigkait schitt ${ }^{331}$. Auch in den Wohnungs- und Arbeitsräumen, in denen sich viele Menschen nebeneinander aufhielten, müsse man sich besonders um die Reinlichkeit sorgen, damit dadurch keine Luftvergiftung entspringe: das in disen leüffen ain yetlicher/der mit vil haußgesind beladen ist/die dann in ainer stuben den wintter arbaiten müssen/da dann vil böß geschmacks in dem selben gemach wirt/ der in disen leüffen gro $\beta$ ursach gibt/ wann aim schmeckt der atem/ das ander ist nit sauber/ das drit hat ain andern mangel/ das dann den lufft der wonung gantz vergifft/ sol man also verhüten/ das in solchen leüffen kains von andern geschedigt wird in den stuben ${ }^{332}$. Abgesehen von dem üblichen Gebrauch der Räucherwerke solle man zusätzlich bei einem weit vom Ofen entfernt gelegenen Fenster eine Scheibe herausnehmen und dadurch ein Dampfloch fertigen, damit die Wärme des Ofens den ganzen Dampf aus dem Raum hinaustriebe.

Auf die Gassen sollen dagegen keinesfalls Harn-, Spül- oder Kräuterwässer geschüttet werden, weil das insbesondere bei der warmen und feuchten Luft deren allgemeine Vergiftung verursache. Auch des Wäschehängens auf den Straßen gelte es sich in der Pestzeit zu enthalten. Wiewol es mit befleckter waesch kains wegs zugedulden ${ }^{333}$, sei es ebenso gefährlich, die gesamte andere Wäsche draußen aufzuhängen. Diese könne nämlich die unrainen feüchtigkait der Menschen in sich aufnehmen und dann kumpt durch wirckung der Sonnen solche unraine unsauberkait auß dem gewand in den lufft/ auß dem lufft in den menschen/ so den lufft an sich ziehen ${ }^{334}$.

Unter den Prophylaxevorschlägen gab es auch eine Gruppe von Anweisungen, die sich vornehmlich auf die Reinhaltung der Luft im öffentlichen Raum bezogen. Die Augsburger Stadtärzte rieten nämlich dazu, dass an einigen Orten in der Stadt Rauchwerke mit brennendem Eichen- oder Tannenholz aufgestellt werden sollten, um damit die Pestepidemie wirksamer abwehren zu können. Dagegen findet sich in der Pestschrift von Michael Fencken der Hinweis, dass man die Verstorbenen in der Pestzeit besonders schnell begraben müsse.

\footnotetext{
331 OCCO/SAYler/TiEfFEnBACh/TalHauser, Was die Pestilentz an jr selbs sey [Augsburg: Philipp Ulhart 1535], S. B I.

${ }^{332}$ JUNG, Ain nutzliche trostliche und kurze underrichtung (1) [Augsburg: Silvan Ottmar 1521], S. A IIv.

333 OCCO/SAYLER/TIEFFEnBACH/TALHAuser, Was die Pestilentz an jr selbs sey [Augsburg: Philipp Ulhart 1535], S. B Iv.

${ }^{334}$ Ebd.
} 
Die Begräbnisplätze sollen von den Leuten möglichst gemieden und daher am besten außerhalb der Stadt angelegt werden. Nach der Ansicht von Fencken gehörte es zu wichtigster Aufgabe der städtischen Obrigkeit, sorgfältig Aufsicht über das Begräbniswesen auszuüben: Darumb ein Obrigkait inn einer Stat/ in solchem fall sonderlich geflissen sein soll die Stat aller ort/ als verr es muglich ist/ auffs sewberst zu halten/ die Cörper außerhalb der Stat zubegraben/ und allen übelen geschmack zuverkommen ${ }^{335}$. Außerdem rieten die Augsburger Stadtärzte ungelöschten Kalk in die Gräber zu schütten, wodurch am frueling vil ungelücks abgewendet werde ${ }^{336}$. Auch in die Aborte sollten die Leute einmal pro Woche bis zu drei Hand voll Kalk werfen, denn der weret dem gestanck/ und nimbt die feülung gantz wunderbarlich ${ }^{337}$.

\section{Ansteckungsgefahr, die von den Pestkranken ausgeht}

Unter den vorbeugenden Maßnahmen, welche laut den Pesttraktaten zum Schutz vor der Vergiftung der Luft zu ergreifen seien, finden sich auch einige Anweisungen, die zum besseren Schutz vor einer Ansteckung mit der Pest dienen sollten. Im Allgemeinen solle man alle großen Menschensammlungen an öffentlichen Orten meiden, weil sich dort die giftige Materie leicht von einem auf den anderen übertragen könne: Item zu meiden ist die versamlung des volcks/ in kirchen/ auff dem marckt/ in weinhäusern/ in badstuben ${ }^{338}$. Hingegen forderten die Augsburger Stadtärzte ausdrücklich, dass die Leute, die sich von der Pest erholt haben oder jene, die diese in ihren Häusern unterbringen, noch einige Wochen nach dem Ende der Seuche zu Hause bleiben und nicht gleich unter die Leute gehen sollen: die/ so an dieser kranckhait gelegen/ in etlichen wuchen/ nach dem die vergifftung gro $\beta$ gewesen/ nit auf die gassen/auch die so schon nit gelegen/doch bey den krancken im hau $\beta$ gewesen waeren/ sollten sich gemainer versamlung/Bad/ Maerckt/ Kirchen/ und andrer volckreichen orten massen ${ }^{339}$. Als gefährlich wurde es auch angesehen, wenn viele Leute gleichzeitig auf einen Abort (ain sprachhau $\beta$ ) gehen oder wenn die Gesunden solche Orte aufsuchten, wo zuvor ein befleckter gegangen sei.

\footnotetext{
${ }^{335}$ FENCKEN, Ain gut nützlich Regiment [Augsburg: Alexander Weißenhorn 1533], S. A IVv.

336 OcCO/SAYLER/TIEFFEnBACH/TALHAuSER, Was die Pestilentz an jr selbs sey [Augsburg: Philipp Ulhart 1535], S. B II.

${ }^{337}$ Ebd.

${ }^{338}$ ZWICKER, Ein kurtze wolgegründte underrichtung [Augsburg: Silvan Ottmar 1537], S. A III.

339 OCCO/SAYLER/TIEFFEnBACH/TAlHAusER, Was die Pestilentz an jr selbs sey [Augsburg: Philipp Ulhart 1535], S. B Iv.
} 
In diesem Zusammenhang stellte Doktor Michael Fencken aus Ingolstadt einige Bemerkungen über die medizinische Praxis in der Pestzeit auf ${ }^{340}$. Nach seinen Beobachtungen würden die Ärzte häufig zu den Kranken gerufen, deren Erkrankung für alle Mitbewohner schon seit längerer Zeit erkennbar sei. Daher sei es besser, wenn die Ärzte solche Kranken nicht mehr persönlich besuchen, sondern nur deren Harnwasser untersuchen. Dann können sie den Kranken eine mündliche Beratung erteilen, welche Medikamente aus der Apotheke zu beschaffen seien. Nach Fencken sei es eine Beschränkung des ärztlichen Kontaktes mit den Pestkranken notwendig, weil durch Doctores embsige visitation/vil leut inficiert unnd vergifft werden/ angesehen das sie zu mancheerlay personen/ so mancherlay wey $\beta$ vergift sein/ geen $^{341}$. Zwar können sich die Mediziner mit verschiedenen Präventivmitteln versehen, die sie vor der Erkrankung schützen, aber trotzdem mögen sie doch durch klaidung/ oten/ contact oder greifen der krancken menschen/ ander leut inficieren/vermackelen und vergifften ${ }^{342}$. Zusammenfassend stellte Fencken nachdrücklich fest, dass der persönliche Kontakt mit dem Arzt wegen der großen Ansteckungsgefahr vermieden werden solle: Ich wolt für mein person/ den Arzt fliehen/ personlich nit bey zuwonen/ der zuzeyten dieser grewsenlichen erschröckenlichen kranckhait/ vil krancker in eygner person haimsuchet/ doch mich seines rats dannoch nit gar verzeyen ${ }^{343}$. Eine schützende Vorgehensweise wurde aber von den Augsburger Doktoren mehrheitlich abgelehnt. In ihren Traktaten wiesen sie dagegen ausdrücklich darauf hin, dass man sich bei der Erkrankung immer auf die direkte Beratung durch erfahrene Ärzte verlassen solle: Darumb sol man auf das fürderlichest/ solche kranckhait got/ und nachmal dem menschen anzaigen oder offnen/ die dann zu solchen dingen verordnet seind ${ }^{344}$. Auch während der Behandlung der Pestkranken gelten weitere ärztliche Untersuchungen als unerlässlich, weil die Medikamente immer nach der individuellen Verfassung eines Kranken zu verordnen seien: derhalben soll man nach aygenschaft der kranckhait des kranckens/ alters und andrer zufall au $\beta$ radt aines verstendigen artzets ain artzney nehmen/ die der natur kain gewalt thue/ sonder sanfft und ring sey ${ }^{345}$. In der städtischen Pestbewältigung in Augsburg war eine direkte Beratung von Ärzten während der Diagnosephase und der Behandlung der Kranken ebenfalls von größter Bedeutung ${ }^{346}$.

\footnotetext{
${ }^{340}$ FENCKEN, Ain gut nützlich Regiment [Augsburg: Alexander Weißenhorn 1533].

${ }^{341}$ Ebd. S. A Vv.

${ }^{342}$ Ebd.

${ }^{343}$ Ebd.

344 JUNG, Ain nutzliche trostliche und kurze underrichtung (1) [Augsburg: Silvan Ottmar 1521], S. B II.

345 OCCO/SAYleR/TIEFFEnBach/TAlHauser, Was die Pestilentz an jr selbs sey [Augsburg: Philipp Ulhart 1535], S. E III.

${ }^{346}$ Siehe S. 146-147.
} 


\section{Lebensführung in der Pestzeit}

Unter den weiteren Anweisungen von Medizinern befanden sich vor allem die Grundsätze zur richtigen Lebensführung in der Pestzeit. Darunter waren die Vorschriften zur gesunden Diätetik von größter Bedeutung. Hierbei galt die Grundregel, dass die Nahrung leicht verdaulich sein und keine überflüssigen Feuchtigkeiten im Leib verursachen solle. Aus diesem Grund empfahl man ausdrücklich, sich beim Essen zu mäßigen und auf einige bestimmte Speisen vollständig zu verzichten. Dazu gehörte vor allem Obst, welches man mit wenigen Ausnahmen völlig vermeiden solle: alle frücht voll böser feüchtigkait seyen/ und wann sy im leib zerstoert werden/ das sy ain natur ainer schedlichen artzney an sich nehmen $^{347}$. Nach Erkenntnis der Augsburger Stadtärzte läge darin sogar eine der wichtigsten Ursachen für die Pesterkrankungen unter dem gemeinen Volk in Augsburg. Abgesehen von der Luftvergiftung und der schlechten Hygiene sei gerade der Verzehr feuchthaltigen Obste für die Verursachung der Pest verantwortlich: wir achten das unserem gemainem volck nit grösserer schad widerfar/ dann das sy das obs brauchen/ und in versunckenen orten staetig wohnen/ auch die selben fast unrayn halten/ sonderlich so der boeß feücht lufft darzu dienet $^{348}$. Außer Obst wurden auch Schweinfleisch, Fische, Wasservögel, Milch oder Pilze als ungesund angesehen. Wenn Fische dennoch gegessen werden, müssen sie aus klarem, fließendem Wasser stammen und ganz frisch gefangen verzehrt werden. Auch von den verschiedenen Fleischsorten wurden nur die gut verdaulichen empfohlen, wie z.B. Geflügel, junges Lamm- und Kalbfleisch oder auch Wild. Hingegen riet man zum häufigen Gebrauch von Essig, dem eine den Körper von schädlichen Stoffen reinigende Wirkung zugeschrieben wurde. Daher sollten alle Speisen grundsätzlich unter Einsatz von Essig zubereitet werden.

Zum Trinken wurde dagegen vor allem ein leichter wohlriechender Weißwein, Wermuth oder Wacholderbeerenwein empfohlen. Man könne sich auch ein Getränk aus Zimtrinde, Anis oder Galgantwurzel sowie einen Most aus Erbsen und Wacholderbeeren zubereiten, so die Anweisung der Traktate. Als schädlich galten ihnen hingegen trübes übelschmeckendes Wasser, dickflüssiges Bier oder ein junger bzw. dickflüssiger Rotwein. Gleichfalls habe man sich des Trinkens von Roséweinen wie auch der weißen Reibsorten Malvasier und Muskat zu enthalten.

\footnotetext{
347 OCCO/SAYleR/TIEFFEnBACH/TAlHauser, Was die Pestilentz an jr selbs sey [Augsburg: Philipp Ulhart 1535], S. B III.

${ }^{348}$ Ebd. Zu dieser ärztlichen Erkenntnis auch der folgende Abschnitt, S. A IIIv: die schon arbayten irer narung halben/ müssen doch in versuncken tempfigen orten sitzen und wohnen/ auch vil von hagelschlechtigem unzeytigem taygem ops und andren feüchten früchten und kreütern esse/ wie bey uns in den tuncken und an andern orten das gemain volck on aufmercken auf sich selbs thut.
} 
Beim Essen und Trinken solle man Mäßigkeit halten und sich jederzeit schädlicher Völlerei enthalten, denn diese führe zur Ansammlung überflüssiger Säfte im Körper. Diese können in der Folge die körperliche Beschaffenheit so verderben, dass man weder mit Purgatzen noch Aderlassen bey solchen füllern und fraeßlingen moege frucht schaffen ${ }^{349}$. Auch alle körperlichen Ausscheidungen des Leibes wie Harn, Stuhlgang und Schweiß sollen ihren natürlichen Ausgang finden. Wenn sie aber zu lange im Körper gehalten würden, führe dies zur allgemeinen Fäulnis des Körpers. Deswegen solle man bei Bedarf insbesondere den Stuhlgang mit Hilfe von Zäpfchen oder Klistier zusätzlich befördern.

Zur Förderung der Verdauung und somit zur Vermeidung von Fäulnisvorgängen im Körper hielten die Ärzte tägliche Bewegung für unentbehrlich. Dadurch sei auf natürlichem Wege die richtige Verteilung der Säfte und die Reinigung aller überflüssigen Flüssigkeiten im Leib unterstützt. Die körperliche Betätigung solle aber nicht gleich nach dem Essen erfolgen, weil dann die bei der Verdauung entstehenden Schadstoffe im ganzen Körper ausgebreitet würden. Man dürfe sich auch nicht zu stark körperlich anstrengen, um keine übermäßige Erhitzung des Leibes zu bewirken. Vollständiger Müßiggang werde jedoch ebenfalls als ungesund erachtet.

Im Hinblick auf die Erhitzung des Leibes wurde in den Pesttraktaten auch die Praxis des Waschens und Badens des menschlichen Körpers detailliert erörtert. Demnach solle man nicht zu oft baden und wenn, dann erst einige Stunden nach Einnahme der letzten Mahlzeit. Es sei auch darauf zu achten, dass das Bad lauwarm vorbereitet werde und nicht zu lange dauere. $\mathrm{Zu}$ warmes Baden führe nämlich zu unnötiger Hitze im Körper und erweitere zu stark die Hautporen, was der vergifteten Luft einen besseren Zugang zum Körperinneren verschaffen könne. Zu häufiges Baden bringe zugleich überflüssige Feuchtigkeit in den Körper. Ein Dampfbad (schwaißbaden) sei daher besser als ein gewöhnliches Wasserbad. Hingegen galt ein Schwefelbad (schwebelbad) als schädlich. Das Baden solle am Besten im eigenen Haus erfolgen, weil die öffentlichen Badstuben wegen der zusammenkommenden Menschenmengen gefährlich seien: doch huete sich ain yeder vor den gemainen Baedern/ und wa vil leut zusammen kommen ${ }^{350}$.

Auch der Geschlechtsverkehr sei aus medizinischer Sicht in Pestzeiten möglichst einzuschränken oder ganz zu unterlassen, denn vil unkeüschait berait den leib zu der Pestilentz $^{351}$. Die drei wichtigsten Körperorgane Hirn, Magen und Herz würden dabei

\footnotetext{
${ }^{349}$ Ebd. S. B IIIv-IV.

${ }^{350}$ BÖHAM, Ain nutzliche/ vnnd für den gemainen Man/ genugsam gegründte vnderricht [Augsburg: gedruckt von Philipp Ulhart und zum Neudruck aufgelegt von Johannes Vogt dem Jüngeren um 1570; bearbeitet und ergänzt von Wolfgang Talhauser um 1547], S. B III.

${ }_{351}$ OCCO/SAYleR/TIEFFENBACh/TALHAUSER, Was die Pestilentz an jr selbs sey [Augsburg: Philipp Ulhart 1535], S. C Iv.
} 
geschwächt, wodurch der Körper den natürlichen Schutz vor der giftigen Materie verliere. Man solle auch eine gute Ordnung im Schlafen und Wachen halten. Im Allgemeinen erachteten sie den Schlaf bei Tage oder direkt nach dem Essen als ungesund, ebenso wie zu langes Schlafen oder Wachen nicht ratsam sei, weil es nach Maßgabe der Ärzte den Körper schwäche.

Auch aufgrund heftiger Gemütserregungen könne die Krankheit leichter ausbrechen, so die Ansicht der Doktoren. Starke Emotionen wie Zorn, Traurigkeit, Verzweiflung und insbesondere Angst machten die Menschen anfälliger für die Erkrankung, wann die forcht ain grosse geschicklichhait im geblut macht/ solliche kranckhait zuempfahen/ dann solchs allen leyb entrüsten ist/ und nit ain klaine ursach zu solcher kranckhait ${ }^{352}$. Dadurch könne die gesamte Prophylaxe beträchtlich erschwert werden, so die Befürchtungen, so dass man in einem solchen Fall sogar mit den bewährtesten Vorsorgemaßnahmen der Pest nicht sicher entkommen könne. Wenn man sich nämlich schon für die Flucht aus der Stadt entscheide, solle man dabei nicht mit zu großer Furcht handeln. Dann könne auch diese mühevolle Maßnahme nicht den erwarteten Schutz bringen: also auch/wann du gleich weyt zeüchst/ und dein ellend weibisch gemut mit dir furst/ ist der sachen nit geholffen/ du must auch wiltu das dir recht geholffen werde (...) die klainmütigkait ab dem gemut schitten ${ }^{353}$. Daher müsse man sich trotz der schwierigen Zeiten bemühen, ein sonniges Gemüt zu bewahren. Um aber trotz der großen Schrecken einen ruhigen Geisteszustand zu behalten, müsse man sich nicht nur gegenseitig Trost und Hilfe spenden, sondern vor allem fest an Gott glauben und sich hilfesuchend an ihn wenden: sollich forcht au $\beta$ schlag/ mit ainem starcken festen glauben zu got/ ains dem andern beystendig zusein/ und hoffen in got er wird und verhieten vor allem

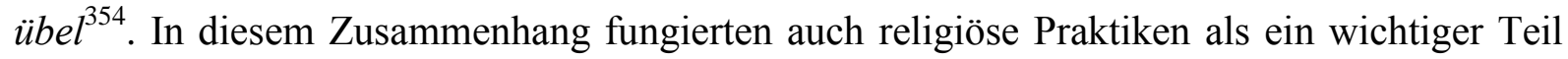
der medizinischen Vorsorgemaßnahmen in der Pestlehre und erfüllten darin eine wichtige Funktion bei der Angstbewältigung in der Pestzeit.

\footnotetext{
352 JUNG, Ain nutzliche trostliche und kurze underrichtung (3) [Augsburg: Silvan Ottmar 1539, Valentin Ottmar 1563], S. B Iv.

353 OCCO/SAYleR/TIEFFEnBach/TAlHauser, Was die Pestilentz an jr selbs sey [Augsburg: Philipp Ulhart 1535], S. B I.

${ }^{354}$ JUNG, Ain nutzliche trostliche und kurze underrichtung (1) [Augsburg: Silvan Ottmar 1521], S. A IIIv.
} 


\section{Prophylaktische Selbstmedikation}

In den meisten Pestschriften wurden zahlreiche Rezepte in deutscher und lateinischer Sprache abgedruckt, die als Anleitung für die selbständige Herstellung spezieller Prophylaktika zum täglichen Gebrauch dienen sollten bzw. auf deren Grundlage man sich die Medikamente in einer Apotheke zubereiten lassen konnte ${ }^{355}$. Durch die regelmäßige Einnahme solcher Arzneimittel werde vor allem die Reinigung des Leibes von überflüssigen Säften weiter unterstützt und das Herz sowie die gesamte körperliche Konstitution eines Menschen verstärkt, so dass die giftige Materie aus der Luft nicht so leicht in dessen Körper eindringen könne.

Die Medikamente wurden dabei in reichhaltiger Form angeboten: man konnte sie nämlich als Latwerge (Arznei in Breiform), Konfekte, Oblaten oder in flüssiger Form einnehmen. Aus der großen Auswahl der Prophylaktika waren aber nicht alle zu gebrauchen, sondern man solle nur so viel davon verwenden, wie man erwerben könne. Wenn es allerdings jemandem finanziell möglich sei, solle er jeden Tag eine andere Arznei verwenden, weil der Körper sich an dasselbe Medikament leicht gewöhne und dadurch die heilende Wirkung vermindert werden könne ${ }^{356}$. Daher wurden die empfohlenen Arzneien meistens für sieben Tage in Folge angegeben, wodurch man den täglichen Wechsel innerhalb einer Woche leichter einhalten konnte.

In den Rezepten für die Prophylaktika werden immer einige Bestandteile genannt, die als besondere Gegenmittel gegen die Pest anerkannt waren. Das wichtigste Heilmittel war „Theriak“, der ursprünglich von Galen in seinem Werk „De antidotis“ beschrieben wurde ${ }^{357}$. Dieses altertümliche Medikament bestand insgesamt aus ca. 70 Kräutern, Wurzeln und anderen organischen Bestandteilen. Eine ähnliche Wirkung wie Theriak schrieb man auch einem anderen Mittel mit Namen „Mithridiatum“ zu, das ebenfalls aus zahlreichen heilsamen Stoffen erzeugt wurde. Daneben benutzte man auch eine rötliche Tonerde namens „Bolus armenum“, deren besondere Eigenschaften der Pest entgegenwirken sollten. Diese drei Arzneien galten allgemein als die bewährtesten Heilmittel in der Pestzeit und wurden von den

\footnotetext{
${ }^{355}$ In den Apotheken, die während der Pestzeit eine wichtige Rolle bei der medikamentösen Versorgung spielten, sollte man die Medikamente nach den in den Pesttraktaten abgedruckten Rezepten, vor allem den lateinischen, bestellen: inn solicher not nit müglich sey/ one hilff der Apotegken/ etwas nützlichs zusamen zutragen/ dann daselbs findt man alle ding schon beraitet/ deßhalben wa ainer inn disem buechle etwa ain artzney finde/ die er vermainte zu der sachen nutzlich zusein/der hat ye nit schwaer am Buechle zutragen biß zu dem Apotegker/dem mag er mit dem finger zaigen/was er woelle gemacht haben, in: BÖHAM, Ain nutzliche/vnnd für den gemainen Man/ genugsam gegründte vnderricht [Augsburg: gedruckt von Philipp Ulhart und zum Neudruck aufgelegt von Johannes Vogt dem Jüngeren um 1570; bearbeitet und ergänzt von Wolfgang Talhauser um 1547], S. A IIv.

356 JUNG, Ain nutzliche trostliche und kurze underrichtung (1) [Augsburg: Silvan Ottmar 1521], S. B I.

${ }^{357}$ Dazu: WINKLER, Galens Schrift; WATSON, Theriac and Mithridatium.
} 
Medizinern sowohl zur Prophylaxe als auch zur Behandlung der Pestkranken ausdrücklich empfohlen. Außer diesen berühmten Gegenmitteln setzten sich auch die Rezepte für Prophylaktika mehrheitlich aus zahlreichen Kräutern, Wurzeln und Gewürzen zusammen, deren Anzahl und Verhältnis stark variieren konnte. Unter diesen Ingredienzien wurden vorrangig die folgenden Heilpflanzen und Substanzen verwandt: Alantwurz, Ampfer, Baldrian, Bibernell, Blutwurz, Boretsch, Feigen, Kletten, Lorbeer, Majoran, Mangold, Muskatnuss, Nelken, Ochsenzunge, Rhabarber, Schöllkraut, Wacholder, Walnuss, Weinraute, Weißwurz, Zitronen, Zitterwurzel.

$\mathrm{Zu}$ den beliebtesten Vorsorgemitteln gegen die Seuche gehörten insbesondere die Pestpillen („Pestilentz pillulen“ oder „Pillas Ruffi“ genannt), die aus Aloe, Ammoniak, Myrre und Safran zubereitet wurden ${ }^{358}$. Daneben konnte man in den Apotheken viele fertige Medikamente für die Pestprophylaxe erwerben, darunter am häufigsten die folgend genannten: „Latwerge aus Ei“, Oblaten „Manus Christi“, „Terra sigilata“ und „De gemmis frigi““ sowie Konfekten „Confectio Liberantis“ und „Confectio cordialis“. Die Armen benutzten gewöhnlich zum Schutz vor der Pest Knoblauch, der daher auch als ,theriaca des gemainen manns“ bezeichnet wurde ${ }^{359}$.

Die Doktoren empfahlen zugleich allen Menschen eine spezielle Reinigung des Körpers, die die überschüssigen Körperflüssigkeiten entfernen sollte und somit eine als sehr wirksam erachtete Prophylaxemaßnahme gegen die Pest darstellte. Auch bei genauer Beachtung aller Regeln zur gesunden Lebensführung käme es nämlich immer noch zur Ansammlung schädlicher Materie im Leib, wodurch einer der Körpersäfte im Überfluss vorhanden wäre: wenn sich der mensch schon gleich wol haltet/ mit vermeydung aller schedlichen ding/ so vil müglich ist/ noch kann er on überfluß nit lang sein/ es mehret sich durch taeglich essen und trincken/ inn etlichen das gebluet/ so vil und fast/ das gefahr dardurch mag entstehn/ inn mancherlay weiß/ so mehret sich in etlichen die Gall/ die dann auch ain ursach ist vilfaeltiger boeser und gaeher hitziger zufaelle/ Etwa mehret sich im menschen vil Phlegma/ und waesserige oder schleymige feüchtigkait/ So nimbt inn etlichen Melancholia überhand ${ }^{360}$. Aus diesem Grund müsse jeder Mensch nach seiner individuellen Beschaffenheit von dieser schädlichen Flüssigkeit gereinigt werden. Diese Maßnahme sollte dabei strikt nach den Anweisungen eines Arztes erfolgen, der für jede Person ein besonderes

\footnotetext{
358 OCCO/SAYler/TiEfFEnBACh/TalHauser, Was die Pestilentz an jr selbs sey [Augsburg: Philipp Ulhart 1535], S. C IIIv.

${ }^{359}$ Ebd., S. B IIv.

${ }^{360}$ BÖHAM, Ain nutzliche/ vnnd für den gemainen Man/ genugsam gegründte vnderricht [Augsburg: gedruckt von Philipp Ulhart und zum Neudruck aufgelegt von Johannes Vogt dem Jüngeren um 1570; bearbeitet und ergänzt von Wolfgang Talhauser um 1547], S. B III.
} 
medizinisches Vorgehen bestimmte. $\mathrm{Zu}$ diesem Zweck verordneten die Mediziner Behandlungen wie den Aderlass, purgierende und schweißtreibende Mittel bzw. Spezialmaßnahmen wie das Fasten oder auch körperliche Bewegung. All diese komplexen Anwendungen sollten auf die individuellen Bedürfnisse und Beschaffenheiten der jeweiligen Patienten zugeschnitten werden, d.h. der Arzt fertigte jeweils einen speziellen Behandlungsplan zur Prophylaxe für jede ihn um Hilfe ersuchende Person an: man muß den handel richten nach gelegenhait ains yeden leibs/ sollen nit den kindern/ schwangern frawen/ und unterschidnen leiben on unterschid artzney geben werden ${ }^{361}$. Weil diese medizinischen Maßnahmen immer aufgrund der spezifischen Vermischung der Säfte eines Menschen zusammengestellt wurden, finden sich in den Pestschriften meistens keine näheren Angaben dazu. Einige Bemerkungen betreffen nur die Ausführung des prophylaktischen Aderlasses, welcher aber ebenfalls nur nach eingehender Beratung durch einen Arzt durchgeführt werden sollte. Die Doktoren warnten ausdrücklich davor, eine derartig komplexe Reinigung des Leibes keinesfalls von Landfahrern oder ungelernten Heilern durchführen zu lassen. Ihrer Überzeugung nach können einzig und allein die gelehrten Ärzte aufgrund ihrer Kenntnis der medizinischen Schriften und ihrer Erfahrung jene Körperzeichen treffend erkennen, die anzeigten, welche Flüssigkeit im Körper jeweils überhand nehme und mit welchen medizinischen Maßnahmen diese am wirksamsten zu vertreiben sei $^{362}$.

\footnotetext{
361 OCCO/SAYler/TIEFFENBACH/TALHAUSER, Was die Pestilentz an jr selbs sey [Augsburg: Philipp Ulhart 1535], S. C III-IIIv.

${ }^{362}$ Ebd., S. C III: Des alles bedarff ains gelerten artztes der künde durch geschribne und erfarne zaichen erkennen/ und nit durch eyteln erdichten berhumb sich verwehne/ welche feüchtigkait des leibs überhand hab/ auch welche Ader zu öffnen sey/ dann es ist nit yederman zulassen in ainer gmain/ wie es auch nit yederman sol verbotten werden on unterschaid; BÖHAM, Ain nutzliche/ vnnd für den gemainen Man/ genugsam gegründte vnderricht [Augsburg: gedruckt von Philipp Ulhart und zum Neudruck aufgelegt von Johannes Vogt dem Jüngeren um 1570; bearbeitet und ergänzt von Wolfgang Talhauser um 1547], S. B Iv: Ain gelerter arrtzet wirt wissen/was ainer yeden natur oder complexion zu rainigung mag am nützlichsten sein/welcher aber sich an die schwaetzer/Landfarer/ oder an die jhenigen so sich für artzet außgeben/ unnd etlicher sonderer künsten beruemen/lassen will/ der sehe war mit er umbgang/ Es seind des betrugs genugsame exempla am tag/ wenn man die nur bedencken will.
} 


\section{Belehrungen von protestantischen Theologen zur Flucht und zum Selbstschutz vor der Pest}

Obwohl die Mediziner eine große Auswahl an Prophylaxemaßnahmen anboten, galt in der Pestzeit allgemein, das man nit bessers mag haben dann die flucht au $\beta$ dem pestilent ${ }^{363}$. Der Rückzug aus dem verpesteten Ort in die gesunde Luft der Umgebung wurde als das sicherste Mittel gegen die Pest bewertet. Schon für die ersten Pestepidemien im spätmittelalterlichen Augsburg ist die Flucht vor der Pest als eine wesentliche Aktion der Zeitgenossen belegt ${ }^{364}$. Was in der damaligen Zeit theoretisch als die wirksamste Prophylaxe angesehen wurde, erwies sich in der praktischen Umsetzung als eine eher beschränkte und auch nicht immer erfolgreiche Lösung. Bis in die Reformationszeit hinein entschieden darüber vor allem zwei Umstände: zum Ersten wurde die Möglichkeit der Flucht nur von Menschen in Anspruch genommen, die finanziell und organisatorisch imstande waren, ihren Heimatort für längere Zeit zu verlassen. Die meisten Leute mussten aber während der Pestzeit geschäffts oder zerung halben am Ort verbleiben ${ }^{365}$. Zum Zweiten schützte auch das Verlassen der Stadt keinesfalls davor, dass jemand der Pest tatsächlich entkam. Die Pest wurde nämlich des Öfteren von den Flüchtlingen, die bereits mit dem Erreger infiziert waren, mitgenommen und weiterverbreitet, wann offt geschicht das ain mensch fleücht und solich böse materi der pestilentz bey im hat/ darau $\beta$ in denn nach der flucht ankumpt die pestilentz ${ }^{366}$. Dann musste es trotz aller Anstrengungen auch letztendlich zur gefürchteten Erkrankung kommen. Aus diesem Grund empfahlen die Autoren der Pestschriften, dass man die Flucht möglichst schnell antreten solle, damit die giftige Materie noch nicht in den Körper eindringen könne. Dafür müsse man in einen entsprechend weit entfernt gelegenen Ort fliehen, in dem es keine Luftvergiftung gäbe. Auch mit der Rückkehr solle man so lange warten, bis die Pestepidemie im jeweiligen Heimatort wirklich vorbei sei.

\footnotetext{
${ }^{363}$ ENGEL, Tractat von der Pestilentz [Augsburg: Sigmund Grimm und Marx Wirsung 4. November 1518], S. A IIIv; Die Flucht vorz den Kranken und aus der Stadt wurde schon in dem ersten Pestdruck aus Augsburg ausdrücklich beraten, TORMANITA, Vil menschen weren der pestilentz frey (1-2) [Einbl. Augsburg: Günther Zainer um 1472]:

Noch ein mayster dir eynen rat geit

Fluch verr davon unn thu das bey zeit

Wann fliehen ist gar ein sicher ding

Vnd halten etlich doch das gering

Fluch die siechen und auch die stat

Seynen rock sein gewand unn was er hat

${ }^{364}$ Siehe S. 33-37.

${ }^{365}$ ENGEL, Tractat von der Pestilentz [Augsburg: Sigmund Grimm und Marx Wirsung 4. November 1518], S. A IIIv.

${ }^{366}$ Ebd.
} 
Neben den genannten Umständen entstand im Zuge der Reformation noch eine weitere religiös begründete Beschränkung der Flucht vor der Pest, wodurch diese Handlungsoption teilweise in Frage gestellt wurde. Die protestantischen Theologen setzten sich nämlich grundsätzlich mit etlichen Fragen den Umgang mit der Pest betreffend sowie mit den damit zusammenhängenden Problemen auseinander ${ }^{367}$. Vom Standpunkt ihres christlichen Glaubens aus erörterten sie vornehmlich die Frage, ob die Flucht vom Wirkungsort der göttlichen Strafe und das Verlassen der leidenden Betroffenen mit der Haltung eines frommen Christen $\mathrm{zu}$ vereinbaren sei. $\mathrm{Zu}$ diesem Thema verfasste Martin Luther (1483-1546) erstmals im Jahre 1527 auf Anfrage einiger Breslauer Prediger und unter dem Eindruck der Pestepidemie in Wittenberg eine gedruckte Schrift, die schon kurz darauf in zahlreichen Nachdrucken erhältlich war $^{368}$. In Nürnberg veröffentlichte dagegen der evangelische Theologe Andreas Osiander (1498-1552) im Jahr 1533 auf der Grundlage des Psalms 91,1-8 eine Pestpredigt zu derselben Frage ${ }^{369}$. Diese wurde ein Jahr später auch in Augsburg gedruckt. In beiden theologischen Pestschriften steht jeweils das Problem der Pestflucht im Zentrum der Ausführungen. Die protestantischen Theologen nahmen die Position ein, dass man vor der Pest nur dann fliehen dürfe, wenn man dabei nicht gegen Gottes Wort verstieße. Nach ihrer religiösen Auslegung entstehe die Pest als Folge einer göttlichen Strafe, weil sich die Menschen sündhaft verhielten. Demzufolge müsse der Mensch zunächst von seinen Sünden ablassen, büßen und seine Lebensweise verbessern, um von dieser göttlichen Plage errettet und vor ihr behütet zu werden. Wenn nun aber die Menschen aus übermäßiger Furcht die Kranken und Bedürftigen ohne die nötige Hilfe verließen, sei ein solches Verhalten ein Akt des Unglaubens und ein Verstoß gegen die evangelische Nächstenliebe, wodurch man Gottes Zorn und Strafe noch vermehren könne. Denn nach den christlichen Geboten sollen sich die Menschen um ihre notleidenden Nächsten sorgen und ihnen alle notwendige Hilfe verschaffen. Aus diesem Grund forderten diese Theologen alle Gläubigen dazu auf, dass sie vor ihrer Flucht erwägen sollen, ob sie je nach Lage ihrer individuellen Verhältnisse nicht zunächst verpflichtet seien, ihren Nächsten Hilfe zu leisten.

\footnotetext{
${ }^{367}$ Von den katholischen Theologen sind für diese Zeit keine Druckschriften über die Pestflucht überliefert. Zum Thema der sogenannten ,theologischen Pestschriften“ in: LANG, Erklärung, S. 133-143.

${ }^{368}$ LUTHER, Ob man vor dem sterben fliehen möge [Wittenberg 1527].

369 OSIANDER, Wie und wohin ein Christ fliehen soll [Nürnberg 1533]. Im Psalm 91, auf den sich in seinen Erläuterungen auch Luther bezieht, wird der starke Glaube als der sicherste Schutz vor der Pest betont. Dieser Bibeltext gewann nach dem Erscheinen der Pestpredigt von Osiander eine besondere Beliebtheit unter den Augsburger Protestanten, worauf die zwei anderen in Augsburg gedruckten Übersetzungen des Psalms, die speziell zum Gesang in der Gemeinde gedacht waren, hinzuweisen scheinen: MUSCULUS, Der ain vnd neintzichst Psalm tröstlich in der Gemain zu der Zeyt der Pestilentz zu singen [Augsburg: um 1540]; BILD, Der Ain vnnd neützig Psalm [Augsburg: Heinrich Steiner 1544].
} 
Wenn jemand beispielsweise seine Verwandten zu pflegen oder sich um seine bedürftigen Freunde und Nachbarn zu sorgen habe, ein geistliches oder weltliches Amt innehabe oder in einer Dienstverpflichtung wie z.B. der eines Knechtes oder einer Magd stehe, müsse er wegen seiner unterschiedlichen Verpflichtungen gegenüber den Nächsten trotz der Pest vor Ort ausharren. Man könne sich in solchen Fällen nur dann an einen Fluchtort begeben, wenn Sorge dafür getragen worden sei, dass die Kranken von anderen Pflegern behandelt oder die amtlichen Pflichten von dazu verordneten Vertretern korrekt versehen würden. Diesen theologischen Erklärungen nach sollte die Pestflucht nicht generell verboten, aber nur in bestimmten Situationen erlaubt werden.

Diese einschränkende Stellungnahme der protestantischen Theologen resultierte aus ihrer anderen Vorstellung, dass man sein von Gott verliehenes Leben schützen und es nicht ohne Not in Gefahr bringen solle. Ähnlich wie bei der Vernachlässigung der Nächstenliebe können die Menschen schwer sündigen, wenn sie sich selbst nicht vor der Pest schützen und dadurch sterben. Mit einer derartigen Vorgehensweise vertrauten sie nicht auf Gott, sondern versuchten Gott. Sofern Osiander nicht näher auf die medizinischen Anweisungen gegen die Pest einging und mit seiner Pestpredigt allen Menschen, die während der Pestepidemie nicht fliehen konnten, vor allem religiösen Trost spenden wollte ${ }^{370}$, setzte sich Luther mit vollem Eifer für die strikte Anwendung der Pestlehre ein. Mutmaßlich aufgrund seiner Erfahrungen aus der 1526 in Wittenberg grassierenden Pestepidemie, wo sich infolge fehlender medizinischer Versorgung die Seuche offensichtlich leichter ausgebreitet hatte, konnte sich seine positive Einstellung zu medizinischen Maßnahmen herausbilden: denn also ist itzt unser Pestilentz hie zu Wittemberg alleine aus geschmeysse herkommen, Die lufft ist Gott lob noch frisch und rein, Aber aus lauter thumkeinheit und verseumunge hat sie etliche und der wenig vergifft ${ }^{371}$. In seiner Deutung wurden nämlich Arzneien und alle aus der Natur stammenden Heilmittel gegen alle Arten von Krankheiten durch Gott geschaffen, damit die Menschen mit ihnen ihre Körper vernünftig pflegen und ihr Leben behalten. Daher sollen die Leute in der Pestzeit so oft und so viel, wie es ihnen möglich sei, Arzneien gebrauchen und ihnen gegebene medizinische Anordnungen befolgen: brauche der ertzney, nym zu dir was dich helffen kann, rauchere haus, hoff und gassen, meyde auch person und stet, da deiner nehester

\footnotetext{
370 OSIANDER, Wie und wohin ein Christ fliehen soll [Nürnberg 1533], S. 390-391: Darumb will ich solche ding auff dißmal in irem werd und frey bleyben lassen wie alle andre natuerlichen ding, die unser vernunft unterworfen und zu regiern bevolhen sein. Darneben aber will ich den christen, so von ampts wegen oder armut halben oder aus andern redlichen ursachen nicht fliehen koennen oder woellen, iren pesten und hoechsten trost, den sie haben, mit vleiß anzaigen.

${ }^{371}$ LUTHER, Ob man vor dem sterben fliehen möge [Wittenberg 1527], S. 369.
} 
dein nichts bedarff odder auffkomen ist ${ }^{372}$. Während der Pestepidemie wurde eine sorgfältige Pflege der eigenen Gesundheit als umso wichtiger erachtet, weil man ohne diese Pflege nicht nur sich selbst schade, sondern durch die eigene Vernachlässigung auch viele andere Personen mit der gefährlichen Krankheit vergiften könne. Damit verstießen die Menschen gegen die Nächstenliebe und brächten die ganze Gemeinde in Todesgefahr: zu dem ist das noch grewlicher, das ein solcher so seinen leib also verwarloset und der Pestilentz nicht hilfft weren, so viel er kann, mochte damit auch viel ander beschmeissen und vergifften, welche sonst wol lebendig bleiben, wo er seines leibs (wo er schuldig ist) hette gewartet, und wurde also auch schuldig seines nehesten todes und viel mal für Got ein moerder ${ }^{373}$. Insbesondere die Pestkranken selbst sollen keinesfalls unter Menschen gehen, sondern sich so bald als möglich nach Ausbruch und Erkennung ihrer Erkrankung von dem gesunden Teil der Bevölkerung absondern und umgehend medizinische Hilfe ergreifen. Diejenigen Infizierten, die mit Absicht andere Menschen vergiften wollten, weil sie glaubten, sich selbst durch die Übertragung der Pest auf andere davon befreien $\mathrm{zu}$ können oder einfach aus purer Schadenfreude die Seuche weiter verbreiteten, sollten entsprechend wie Mörder und Verbrecher verurteilt werden.

Außer um die Körperpflege solle man sich während der Pestepidemie zugleich auch um die Seelenfürsorge bemühen und sich auf den möglichen Tod vorbereiten, so Luther. Die Leute sollen rechtzeitig zur Beichte gehen und die Sakramente empfangen. Mit der Anforderung der Seelsorger zur letzten Ölung der Kranken sei keinesfalls bis zu dem Zeitpunkt zu warten, bis die betreffende Person schon das Bewusstsein und die Sprachfähigkeit verloren habe. Denn dann könne man nicht mehr das Glaubenbekenntnis ablegen, welches jedoch eine unentbehrliche Bedingung sei, um die Sakramente zu erhalten. Anders als die katholischen Priester sollen nämlich die protestantischen Prediger ohne entsprechendes Bekenntnis zum Evangelium keine Sakramente an die Kranken erteilen. Man solle sich ebenfalls rechtzeitig in den Zeiten der Pestepidemien um die Ausfertigung von Testamenten oder auch um die Aussöhnung mit Verwandten bemühen.

Darüber hinaus betont auch Luther die Bedeutung der obrigkeitlichen Fürsorge während der Pestepidemien. Ein spezielles Spital oder einige Häuser, in denen die Pestkranken gepflegt und versorgt würden, seien eine wesentliche Aufgabe für die christliche Obrigkeit: Wol war ists, wo ein solch stadlich regiment ynn stedten und landen ist, das man gemeine heuser und spital kann halten und mit leuten, die yhr warten, versorgen, da hin man

\footnotetext{
${ }^{372}$ Ebd., S. 365.

${ }^{373}$ Ebd., S. 363.
} 
aus allen heusern alle krancken verordnete (...) das were wol sein, loeblich und Christlich, da auch billich yderman mildiglich zu geben und helffen sollte, sonderlich die oberkeit ${ }^{374}$. Wo aber solche Einrichtungen nicht vorhanden seien, müssten alle Menschen gemeinsam die Pflege ihrer Nächsten in Eigenverantwortung übernehmen.

Unter anderem wegen der als schädlich erachteten Ausdünstungen aus den Gräbern wurde die Errichtung von sogenannten Gottesackern außerhalb der Stadt empfohlen. Dabei sollten jeweils die Ärzte als die medizinischen Experten bewerten, ob die angelegten Begräbnisstätten auf den inneren Kirchhöfen zur weiteren Luftvergiftung des städtischen Raumes beitragen könnten: Auffs erst las ich das die Doctores der ertzney urteilen und alle die des bas erfarn sind, obs ferlich sey, das man mitten ynn stedten kirchhofe hat. Denn ich weis und verstehe mich nichts drauff, ob aus den grebern dunst odder dampff gehe, der die lufft verruecke. Wo dem aber also were, so hat man aus obgesagten warnungen ursachen gnug, das man den kirchhoff ausser der stad habe ${ }^{375}$. Die Verlegung der Kirchhöfe aus der Stadtmitte sei außerdem aus religiösen Gründen zu befürworten. Die Begräbnisstätten sollen nämlich an einem stillen und besinnlichen Ort gelegen sein, wo die Leute mit Andacht beten und sich an Gottes Wort erinnern können.

Nach Luthers Auslegungen gehörte es also zu den Pflichten eines Christen, mit allen zur Verfügung stehenden Mitteln für die Gesundheit und das Leben vorzusorgen. Einerseits sollte man dem evangelische Gebot der Nächstenliebe nachgehen, andererseits sich auch um das eigene Wohlergehen bemühen. Nur unter Beachtung dieser beiden Grundsätze könne man gemäß dem Willen Gottes agieren, der aber letztlich allein darüber entscheide, wer während der Pestepidemie für den Tod oder das Leben bestimmt sei: Will mich mein Got daruber haben, so wird er mich wol finden: so hab ich doch gethan das er mir zu thun gegeben hat und bin widder an meinem aigen nach ander leute tode schuldig. Wo aber mein nehester mein darff, will ich widder stet noch person meiden, sondern frey zu yhm gehen und helffen, wie droben gesagt ist. Sihe das ist ein rechter Gottfurchtiger glaube, der nicht thunkune noch frech ist und versucht auch Gott nicht ${ }^{376}$. Man kann voraussetzen, dass unter dem Einfluss solcher religiöser Begründungen die Pestlehre unter der protestantischen Bevölkerung weiter verbreitet wurde. Die Verbreitung des medizinischen Diskurses wurde also durch die reformatorischen Umwandlungen weiter begünstigt und konnte seit der Drucklegung von Luthers Schrift im Jahre 1527, die schon bald in zahlreichen Nachdrucken im deutschsprachigen Raum zirkulierte, auch auf diese Art und Weise einen breiteren Zugang zur

\footnotetext{
${ }^{374}$ Ebd., S. 353-355.

${ }^{375}$ Ebd., S. 373-375.

${ }^{376}$ Ebd., S. 367.
} 
Vorstellungswelt der Zeitgenossen erreichen. Im Fall Augsburgs fand die Rezeption der Pestlehre jedoch schon am Ende des 15. Jahrhunderts statt, bevor man diese Frage in den theologischen Pestschriften zu diskutieren begann ${ }^{377}$.

Die direkten Auswirkungen der protestantischen Stellungnahme zur Pestflucht und zur Beachtung der medizinischen Anweisungen lassen sich in den Augsburger Pestschriften beobachten. Die Stadtärzte aus Augsburg bemerkten in ihrem Traktat, dass sie im Allgemeinen dem Rückzug aus der Stadt während einer Pestepidemie nicht entgegenstünden. Dafür sollten sich aber nur diejenigen entscheiden, die mit keinerlei Verpflichtungen gegenüber anderen belastet seien: doch woellen wir hie kainem ursach geben haben/ weib und kind/ underthanen oder ander/ die ime amptshalben oder in ander weg bevolhen seind/ zu verlassen/ ain yeder woelle sich hie erinnern/ was und wie weyt er Got und dem nächsten verpflichtet sey ${ }^{378}$. Ebenso wird in der Pestschrift von Adam Zwicker das Problem der Flucht im protestantischen Sinne dargelegt ${ }^{379}$. Auch hier wird zuerst daran erinnert, dass man seinen Nächsten gegenüber christliche Liebe zu beweisen schuldig sei. Jedoch solle man nur dann zu den Kranken gehen, wenn es wirklich notwendig sei. In diesem Traktat wird auch die medizinische Versorgung als ein wichtiger Teil der göttlichen Vorsehung gedeutet. Schon auf dem Titelblatt dieser Druckschrift befinden sich zwei kurze Zitate aus dem Jesus Sirach, in denen die religiöse Deutung der medizinischen Kunst deutlich zutage tritt: Eccls. 12. Es forchte got/ und halte seine gebot/ Alle menschen durch Christum erschaffen/von got/ Auch den artzt von got erschaffen/ ferrer gebrauch dich artzneien so von got kummen her. Eccls. $38^{380}$. Die Einleitung erinnert ebenfalls daran, dass sich die Menschen nach der Ausübung der religiösen Praktiken auch unbedingt zu dem von got verordneten mittel der artzney wenden sollen $^{381}$. Darüber hinaus betonte Zwicker, dass die medizinische Hilfe auch von den christlichen Obrigkeiten organisiert werden solle, indem sie während der Pestepidemien entsprechendes medizinisches Personal für die Bevölkerung anstellen: es gehört einer Christlichen oberkeit allenthalb zu/ und ist ihr ampt/ das sie tugendliche leüt/ und die gnad von got haben/darzu verordnen/ und inen ir speiß und besoldung geben/das sie inen warten/

\footnotetext{
${ }^{377}$ Siehe Kapitel VI, S. 126f.

378 OCCO/SAYleR/TIEFFENBACH/TALHAuser, Was die Pestilentz an jr selbs sey [Augsburg: Philipp Ulhart 1535], S. A IVv-B I.

${ }^{379}$ ZWICKER, Ein kurtze wolgegründte underrichtung [Augsburg: Silvan Ottmar 1537].

${ }^{380}$ Ebd.

${ }^{381}$ Ebd. S. A Iv; Auch dazu: Darumb wirdt ein weiser Christen man nicht verachten das mittel/ das Got der herr dem menschen zu der gesunthait verordnet hatt. Auch Ambrosius Jung betont in seinem Pesttraktat, dass man nach den religiösen Handlungen immer die Naturheilmittel gebrauchen sollte, so der Herr in erfarnuß zu abwendung diser kranckhait menschlicher natur eröffnet und verordnet hat in: JuNG, Ain nutzliche trostliche und kurze underrichtung (2) [Augsburg: Silvan Ottmar 1535], S. A IIIv.
} 
und sie trösten ${ }^{382}$. Die medizinische Versorgung sollte also sowohl auf der individuellen wie auch auf der obrigkeitlichen Ebene unternommen werden. Auch in dem Pesttraktat von Alexander Seitz wurden die Zeitgenossen zur Beachtung des christlichen Gebots der Nächstenliebe in der Pestzeit ausdrücklich ermahnt ${ }^{383}$. Diese 1522 verfasste Pestschrift zeigt dabei deutlich, dass schon in der Zeit vor den schriftlichen Ausführungen Luthers zu diesem Thema, das Problem der Pestflucht von den reformatorisch gesinnten Medizinern erörtert wurde. Seitz erinnerte hier an die Pflichten der Pfarrer, sich um ihre Gemeindemitglieder zu sorgen. Dann wandte er sich an die Obrigkeiten mit der Anweisung, dass sie in der Pestzeit nicht fliehen, sondern über die Anordnung einer Apothekentaxe auch die Armen mit preisgünstigen Arzneien versorgen und neben den Aderlass durchführenden Ärzten auch die gelehrten Doktoren zur medizinischen Hilfeleistung anstellen sollen. Bemerkenswert hierbei sind Seitz' spöttische Bemerkungen über die Tätigkeit von Klöstern, deren Mitglieder seiner Ansicht nach zwangsweise zur Krankenpflege in der Pestzeit verordnet werden sollen ${ }^{384}$. Solche öffentlich geäußerte Kritik an Obrigkeit und Kirche führte vermutlich zu seiner baldigen Ausweisung aus München.

\section{Behandlung der Pestkranken}

In den Abschlusskapiteln der Pestschriften finden sich hauptsächlich Erörterungen der bei den Kranken anzuwendenden Therapien ${ }^{385}$. Aufgrund der damaligen Medizinlehre wurde ein kohärentes Heilverfahren für die Pestkranken ausgearbeitet, dessen wichtigste Grundsätze in allen medizinischen Traktaten beachtet wurden. Die feststellbaren Unterschiede betreffen vor

\footnotetext{
${ }^{382}$ ZWICKER, Ein kurtze wolgegründte underrichtung [Augsburg: Silvan Ottmar 1537], S. A III.

383 SEITZ, Ain schoener Tractat von dem Saturnischen gschoß der Pestilentz [Augsburg: Otmar Silvan, 15. Februar 1522].

${ }^{384}$ Ebd. S. 161-162: Zum andern/ ist wissentlich/ das gar offt in vil conciliis/ die Lolhart und grawen begeinen gaentzlich verworffen sein/ und das man sy kains wegs in der welt dulden soll/ ursach/ vil jung starck personen kummen zu inen/ und allain auß faulkait/sy betlen/sy ziehen andern notürftigen leib armen leüten ir narung auß dem mund/ja man muß inen rent und gilt zutragen/ und also streben/biß man diese unnütz faul muessiggenger ernert/ und doch sy dargegen nit für ain lauß in der welt nütz sein/ seind sy menschen/ so sollten sy auch menschlicher art pflegen/darvon sich nit absündern/ und inn ligen wie die moestschwein/ soellen auch den menschlichen acker treülich helffen buwen (...) Darumb/woelt diß geschlecht/ die lolhart und begeinen/ sich ye in der welt begeen/ das man sy darzu vergeweltigt/ das sy den krancken pflegen/ als sy auch alwegen in den conciliis sich mit solcher mue und treüw iren stand verglimpft haben/man sicht aber das ain verheüter tant ist/ Ich mag ye von gemains nutz wegen nit underlassen anzetasten/wenn man ye in yedem winckel in stetten oder zu feld will bawen kloester/ ja stell/ darinn die faulen leüt zu moesten/wer will zu letst bauwen und die noturfftige commun erneren/warlich es ist nit die bruderschaft/ darzu uns Christus ermant.

${ }^{385}$ Nur ausnahmsweise werden die Ausführungen über die Behandlung der Kranken gleich nach dem Teil über die Ätiologie der Pest dargestellt wie z.B. in KLAINMÜLLER, Ain hymlischen vnn Natürlichen anzaygung [Augsburg: Hans Schönsberger, 12. Oktober 1521].
} 
allem die zeitliche Abfolge der durchzuführenden Heilmaßnahmen, diese blieben aber in ihrer medizinischen Verfahrensweise konstant. Auch die Bevorzugung einiger bestimmter Rezepte für die Erzeugung von Medikamenten war ohne weiterführende Folgen für die gesamte Therapie. Diese geringen Differenzen in den Pestschriften weisen jedoch darauf hin, dass alle Mediziner ihre Behandlungsmethoden nicht nur aus den antiken Schriften gelehrter Mediziner, sondern immer aus eigenen Erfahrungen bezogen, die sie im direkten Umgang mit den Pestkranken erwarben. Dies zeigt zugleich deutlich, dass die Ärzte beständig auf der Suche nach wirksameren Heilmethoden innerhalb des damaligen medizinischen Systems waren, jedoch blieben die Erkenntnisfähigkeiten zu dieser Zeit beschränkt und bestimmte Wahrnehmungsgrenzen der Krankheitsphänomene unüberwindbar.

Bevor die Mediziner in ihren Traktaten die Therapiemethoden darlegten, wurden zunächst die wichtigsten Symptome der Pesterkrankung aufgezählt, die auch als Grundlage für eine korrekte Selbstdiagnose dienen konnten. Vom medizinischen Standpunkt aus war das rechtzeitige Erkennen der Krankheit aus zweierlei Gründen von größter Bedeutung. Wegen des raschen Verlaufs der Pest, die innerhalb kürzester Zeit mit dem Tod enden konnte, musste die entsprechende Behandlung möglichst schnell aufgenommen werden. Nach der Erfahrung der Mediziner verminderten sich die Chancen für eine Genesung beträchtlich, je später die notwendigen Heilmaßnahmen eingesetzt wurden. Andererseits wurden die Pestkranken wegen des ansteckenden Charakters ihrer Krankheit auch als potentielle Gefahr für gesunde Menschen wahrgenommen, weshalb man die Kranken möglichst schnell von ihren Mitmenschen absonderte.

$\mathrm{Zu}$ den wichtigsten Symptomen der Pesterkrankung gehörte vor allem das sogenannte pestilentzische Fieber, welches sich infolge des Eindringens der giftigen Materie ins Herz im ganzen Leib erhebe ${ }^{386}$. Der Kranke bekomme dabei Hitzewellen, Schüttelfrost, rasendes Herzklopfen, Atemnot, Schmerzen im Brustkorb und Schweißausbrüche. Diese Krankheitserscheinungen würden von Übelkeit, Krämpfen und Schwerfälligkeit in allen Gliedern, Appetitlosigkeit und großem Durstgefühl, Schläfrigkeit, Störungen des Magens mit Erbrechen und Durchfall, stinkendem Atem, Blutspeien sowie unnatürlichen Veränderungen der Harnfarbe begleitet ${ }^{387}$.

\footnotetext{
${ }^{386}$ Die ausführlichen Erörterungen über das pestilentzische Fieber befinden sich vor allem in: BöHAM, Ain nutzliche/ vnnd für den gemainen Man/ genugsam gegründte vnderricht [Augsburg: gedruckt von Philipp Ulhart und zum Neudruck aufgelegt von Johannes Vogt dem Jüngeren um 1570; bearbeitet und ergänzt von Wolfgang Talhauser um 1547].

${ }^{387}$ Gleichzeitig als Symptom und Ursache des Fiebers wurden auch nicht näher bestimmte Würmer im Leib der Kranken genannt. Ebd.: auch seind Pestilentzische würm in etlichen menschen/die verursachen vergiffte Fieber/ und unleydlichen Kopffwee/ also/ das etlich unrichtig werden.
} 
Als die typischen Pestzeichen schlechthin galten aber die Apostemata: Beulen und Blattern, die sich meistens in der Achselhöhle, auf der Leistenbeuge oder hinter den Ohren herausbildeten. An diesen Körperstellen konnten die Erkrankten schon vor dem Erscheinen der Beulen ein stechendes Gefühl bemerken. Nach der Vorstellung der Mediziner erschienen die Pestbeulen immer an solchen Körperteilen, bei denen sich die giftige Materie in einem der drei inneren Hauptorgane: Hirn, Herz und Leber am meisten angehäuft habe. Bei der Beschreibung der Pestsymptome bemerkten die Mediziner, dass diese Schwellungen erst in einer späteren Phase der Krankheit zu erkennen seien. Sie erklärten dies damit, dass die Beulen nur auftreten können, wenn die körperliche Konstitution eines Kranken kräftig genug sei, um die giftige Materie noch auszustoßen. In vielen Fällen komme es aber gar nicht zur Herausbildung von Apostemata, weil die Kranken bereits früher infolge des pestilenzischen Fiebers sterben: das gifft dringt zu stundan dem hertzen $z u /$ darumb auch offt solche menschen/ die es also leicht ankumpt/sterben gleich als unversehenlich/ und faren gar selten Peülen/ oder zaichen auf $f^{388}$. Nach der Erkenntnis der Mediziner könne die Pesterkrankung also auch ohne Beulen oder Blattern verlaufen. Aus diesem Grund solle eine sichere Diagnose der Pest keinesfalls nur auf der Sichtung der Pestbeulen beruhen, sondern unbedingt auch die anderen Symptome der Krankheit einschließen. Je mehr derartige Krankheitszeichen wahrzunehmen seien, desto schwerer sei der Körper von der Pest befallen.

Nachdem die Pest sicher diagnostiziert wurde, forderte man die Kranken zunächst, sich mit Bittgebeten direkt an Gott zu wenden. Da die primäre Ursache der Pest nach der damaligen Ätiologie immer als Verhängnis Gottes angesehen wurde, könne die Wiedergenesung auch nur durch Gottes Willen geschehen. Laut dieser Sinndeutung hing die Wirksamkeit der anzuwendenden Therapie immer mit der göttlichen Vorsehung zusammen: wir wollen in disem unserm schreiben uns bezeügt haben/ das wir der artzney kains wegs mehr zugeben wollen/ dann als vil Got der Allmächtig das gedeyhen gibt (...) derhalb unser radt/das man sich anfangs Got dem Allmächtigen gantz und gar ergeb durch Jesum Christum unsern hayland/ mit gantzem herztlichem layd vergangner und aller sünd halben/ auch mit ernstlichem anruffen umb gnad und hilff götlichen willen zu gedulden ${ }^{389}$. Aus diesem Grund sollten die religiösen Handlungen üblicherweise dem Einsatz medizinischer Maßnahmen vorausgehen $^{390}$.

\footnotetext{
${ }^{388}$ Ebd. Dazu auch die folgende Feststellung: dises Pestilentzisch Fieber macht nit in allen krancken Peülen auffaren/ es ligt offt verborgen/ biß die natur gar vergifftet wir/ und will darnach kain mittel helfen.

389 OCCO/SAYleR/TiefFenbach/TalHauser, Was die Pestilentz an jr selbs sey [Augsburg: Philipp Ulhart 1535], S. D I-Iv.

390 Über die Empfehlung von Johannes Engel zur zeitlichen Verschiebung der religiösen Praktiken in der Pestzeit, siehe S. 87.
} 
Die Ärzte betonten dennoch ausdrücklich, dass die medizinische Versorgung spätestens innerhalb von 24 Stunden nach der Diagnose der Erkrankung eingeleitet werden solle. Der rasche Verlauf dieser Krankheit lasse keine zeitliche Verzögerung zu und erforderte daher ein entschiedenes Ergreifen der Mediziner: in dieser grewlichen gifftigen schnellen kranckhait/ die dem hertzen also sehr zuwider/ wa sy nit gantz schnell vom hertzen außgetriben/ und nimmer hindersich gelassen wirt/ das sy auch ganz schnell tod bringt/ auch bey aller stercksten menschen ${ }^{391}$. Die Grundregel der medizinischen Behandlung bestand darin, dass man das Pestgift möglichst schnell aus dem erkrankten Körper austreibe. Dies müsse durch die Abgabe von Blut, Schweiß, das Auftreten von Pestbeulen und Ausscheidungen wie Harn und Stuhlgang erfolgen. Zu diesem Zweck machten sich die Mediziner alle bewährten Heilmethoden der damaligen Medizin zunutze: Aderlass, Arzneimittel und Wundarznei.

In den Pestschriften wird über die Ausführung der therapeutischen Maßnahmen in unterschiedlicher Abfolge beraten. Die Augsburger Stadtärzte verordneten für den Kranken zunächst ein Klistier, das für die Wirksamkeit der weiteren Behandlung von großem Nutzen sei $^{392}$. Anschließend solle man in der Regel einen Aderlass durchführen, weil das Blut aufgrund seines feucht-warmen Charakters die Vergiftung des Körpers beschleunige. Diese Behandlung nahmen die Wundärzte an einigen großen Venen auf den Armen, Händen oder Füßen vor, die immer nach der Platzierung der aufgetretenen Pestbeulen bestimmt wurden.

Die ausführlichsten Hinweise über den Aderlass gibt der Augsburger Doktor Johann Klainmüller in seinem Traktat ${ }^{393}$. Gemäß den damaligen Erkenntnismöglichkeiten deuteten Pestbeulen im Kopfbereich oder auf den Schultern an, dass sich die größte Giftansammlung im Hirn befinde. Im Fall der Apostemata auf dem Kopf müsste man daher die Hauptvene auf dem Arm oder auf der Hand zwischen Daumen und Zeigefinger zur Ader lassen. Die Beulen auf den Schultern erforderten dagegen einen Aderlass der Leber- oder Lungenader an derselben Stelle. Ratsam sei es auch zusätzlich das Blut unter der Zunge zu schröpfen. Die Anschwellungen in der Achselhöhle oder unter dem Arm entstünden aus der Vergiftung des Herzens, daher müsse man die Herz- oder Leberader auf dem Arm ablassen. Wenn die Pestbeulen aber auf der Leistenbeuge oder auf den Beinen aufbrechen, leide die Leber unter dem Gift. Bei Apostemata in der Leistengegend öffnete man dann die Rosenader oder die

\footnotetext{
391 OCCO/SAYler/TIEFFenBach/TalHauSER, Was die Pestilentz an jr selbs sey [Augsburg: Philipp Ulhart 1535], S. D IIIv.

${ }^{392}$ Ebd. S. D Iv: soltu dem krancken von stundan ain Criesterlen geben/ dann im anfang kanstu nichts bessers noch nützrs thun/dann es ist mit beschlossnem leib kains wegs zulassen/auch seind die schwaiß artzneyen nit als gar tauglich wann der leib nit offen/dann sy machen etwa mehr hitz/ treyben mehr übersich in den kopff bö $\beta$ reüch und tämpff/ die sunst durch die Criestier unter sich und von edlen glidern wegk zogen wurden.

${ }^{393}$ KLAINMÜLLER, Ain hymlischen vnn Natürlichen anzaygung [Augsburg: Hans Schönsberger, 12. Oktober $1521]$.
} 
Hauptader an der großen Zehe und bei Pestbeulen an den Beinen die Rückenader an der kleinen Zehe oder die Leberader auf der Hand.

Wenn jedoch keine Pestbeulen auf dem Leib fühlbar seien, solle man am rechten Arm die Leberader oder die Herzader öffnen oder abhängig davon, wo die meisten Schmerzen im Körper empfunden würden, die entsprechende Ader auswählen. Falls hingegen die Apostemata an beiden Körperseiten auftreten, so solle man immer auf der rechten Seite zur Ader lassen, um dadurch das Gift vom Herzen wegzuziehen.

Bei der Anwendung des Aderlasses solle man immer die körperliche Konstitution, das Alter oder die Blutmenge eines Kranken individuell berücksichtigen. Wenn sich jemand der Behandlung wegen Kräftemangels nicht auf einmal unterziehen könne, solle man den Aderlass schrittweise in einigen Zeitabständen durchführen oder sogar ganz darauf verzichten. Bei Kindern bis 12 Jahren, älteren Menschen ab 60 Jahren oder schwangeren Frauen wurde der Aderlass durch das Schröpfen ersetzt, indem man die Haut an den Schröpfstellen mit einem Eisen anritzte. Die Schröpfköpfe wurden auch nach der Beendigung des Aderlasses direkt auf die Beulen oder Blattern oder wenig darunter gesetzt, damit die vom Körper ausgestoßene Materie nicht wieder ins Körperinnere zurückfließen könne.

Abgesehen von solchen Erörterungen werden in einigen Pestschriften anhand von Schaubildern die wichtigsten Aderlassstellen aufgezeigt ${ }^{394}$. Am Beispiel nackter menschlicher Körper werden die typischen Pestbeulen bildlich dargestellt und von ihnen abgehende Pfeile zeigen jeweils genau an, bei welcher Pestbeule welche Ader zu öffnen wäre (Abb. 15-16).

\footnotetext{
${ }^{394}$ KRAUTWADEL, Ein nutzlich Regiment der gesundtheyt [Augsburg: 1531]; ANONYMUS, Ain regiment wider Die Pestilentz [Augsburg: Johannes Schönsberger um 1525]; VERINGER, Ein kurtz nützlichs Regiment [Augsburg: Heinrich Steiner 1533]. In der Pestschrift von Adam Zwicker befindet sich auch eine Darstellung des sogenannten Aderlassmännchens, aufgrund dessen man allgemein ablesen konnte, zu welchen Zeiten, festgelegt nach den Mondphasen in bestimmten Tierkreiszeichen, ein prophylaktischer Aderlass am günstigsten durchzuführen wäre, in: ZWICKER, Ein kurtze wolgegründte underrichtung [Augsburg: Silvan Ottmar 1537].
} 


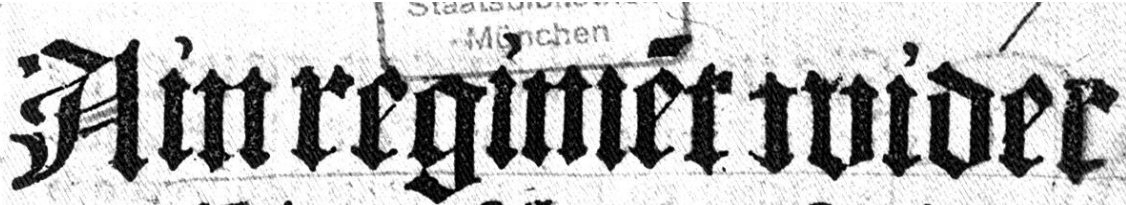 Die peltilentz vait güt}
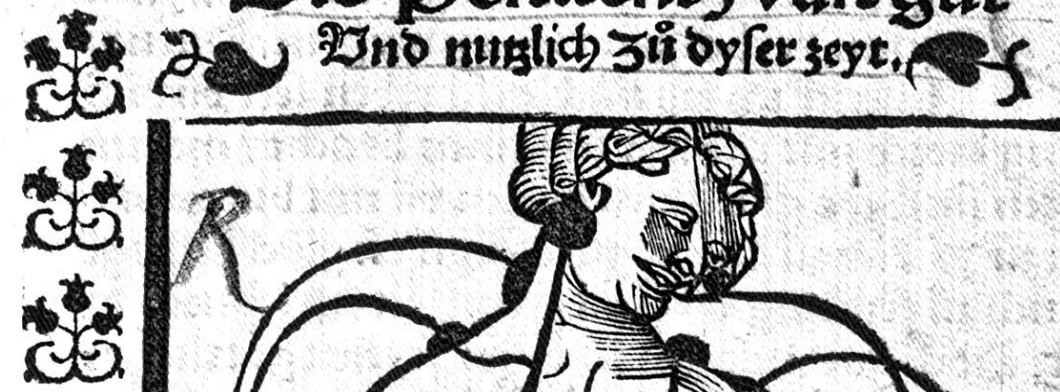

SE

is

\%

do

isto

औ.

s.

st?

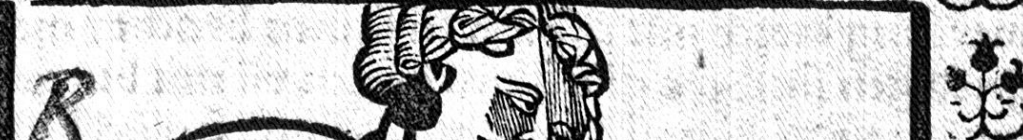

tis

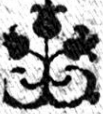

3

36

cte

30
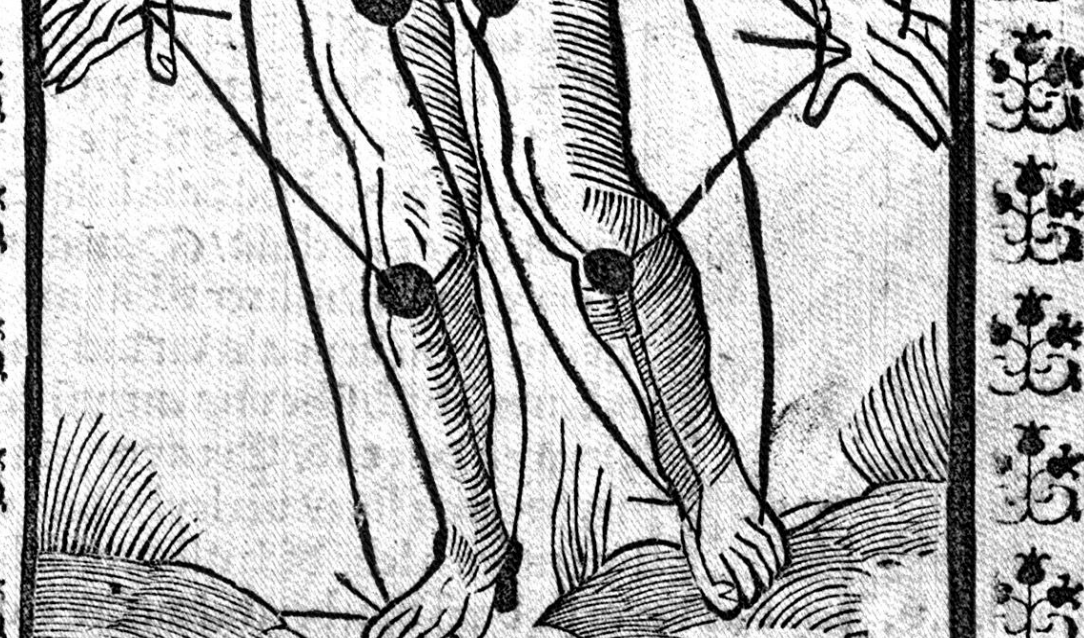

( $2(4)$

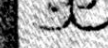

ist

Abb. 15: Aderlassmännchen für die Pestzeit, Titelblatt in: ANONYMUS, Ain regiment wider Die Pestilentz [Augsburg: Johannes Schönsberger um 1525] 


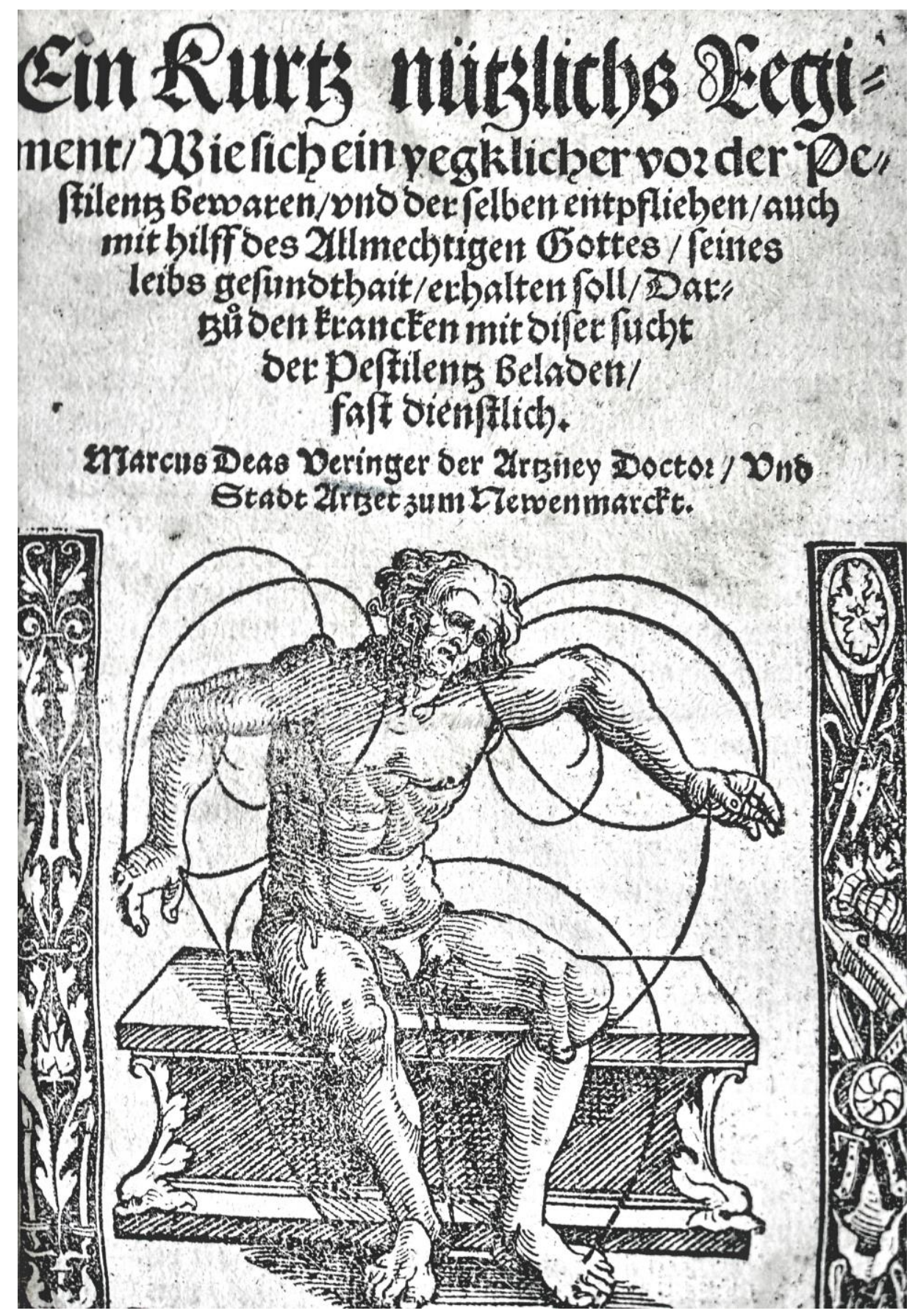

Abb. 16: Aderlassmännchen für die Pestzeit, Titelblatt in: VerINGER, Ein kurtz nützlichs Regiment [Augsburg: Heinrich Steiner 1533] 
Nachdem medizinische Maßnahmen zur Reinigung des Bluts ergriffen worden waren, versuchte man als nächstes über den Schweiß die giftige Materie aus dem Körper auszustoßen. Der Kranke solle ein ihn erhitzendes Arzneimittel einnehmen und dadurch zu starkem Schwitzen angeregt werden. Zu diesem Zweck wurden von den Medizinern zahlreiche Rezepte für die Herstellung von Schweißtränken angeboten. Die Bestandteile der empfohlenen Arzneien stimmen im Allgemeinen mit denen der Prophylaktika überein, wobei dem Theriak auch hier die größte Bedeutung zukommt ${ }^{395}$. Nach der Einnahme eines Medikamentes solle der Kranke bis zu vier Stunden gut zugedeckt liegen und möglichst stark schwitzen. Während dieser Zeit durfe er nicht einschlafen, da sich im Schlaf das Pestgift in das Herz zurückziehe. Zusätzlich könne man dem Kranken auch Konfekt, Oblaten oder Latwerge zur Herzkräftigung reichen.

Nach der Erkenntnis der Ärzte lag aber die durch die Pest verursachte Vergiftung nicht nur im Blut, sondern ebenso in allen anderen Körperflüssigkeiten. Aus diesem Grund müsse noch am gleichen Tag eine spezielle Purgation durchgeführt werden, um die Vergiftung auch aus den übrigen Körpersäften zu entfernen. Diese Behandlung musste jedoch im Gegensatz zur durchgeführten Schwitzkur strikt nach den Anordnungen eines Arztes erfolgen, der nach der Untersuchung der individuellen Beschaffenheit des Kranken die entsprechenden Medikamente dafür verordnete: so nun eingebung der artzney soll beschehen durch radt der gelerten ärtzet/ so wöllen wirs inen bevolhen haben/ und darbey bleyben lassen ${ }^{396}$.

Nach der Anwendung von Klistier, Aderlass, Schwitzen und Purgieren, die in den Pestschriften jeweils in unterschiedlicher Abfolge, je nach den verschiedenen Erfahrungen der Verfasser, angeordnet sind, sollte die Behandlung der Pestbeulen einsetzen. Die Grundregel dieser Maßnahmen bestand darin, dass man die Apostemata möglichst schnell öffnen und reinigen solle. Da man glaubte, dass durch solche Geschwulstbildungen das Gift ausgeschieden würde, durften sie auf keinen Fall wieder ins Körperinnere zurückgetrieben werden, sondern stattdessen hoffte man durch die Anwendung zahlreicher Heilmittel möglichst viele Pestbeulen hervorbringen $\mathrm{zu}$ können. Die Augsburger Stadtärzte unterschieden dabei zwei allgemeine Arten der Apostemata, die auf andere Weise zu kurieren seien: erstlich beülen unnd knöpff, ettwan klain wie die widergeng oder frostbeülen/ ettwan

\footnotetext{
${ }^{395}$ Siehe S. 105-107.

396 OCCO/SAYleR/TIEFFEnBaCh/TAlHAuser, Was die Pestilentz an jr selbs sey [Augsburg: Philipp Ulhart 1535], S. E IIIv; Einige Rezepte für die Purgativmittel, die sich dabei auch aus den üblich angewandten Heilstoffen zusammensetzen, werden dagegen vor der Behandlung der Apostemata beraten, in: BöHAM, Ain nutzliche/ vnnd für den gemainen Man/ genugsam gegründte [Augsburg: gedruckt von Philipp Ulhart und zum Neudruck aufgelegt von Johannes Vogt dem Jüngeren um 1570; bearbeitet und ergänzt von Wolfgang Talhauser um 1547].
} 
ganz groß/zum andern hitzig prennend platern/die zu zeyten schwartz/ rot/gelb/ praun/ oder ainer andern farb seind ${ }^{397}$. Um die Beulen und Blattern besser unterscheiden zu können bzw. zur Verdeutlichung der für sie typischen Erscheinungsstellen am menschlichen Körper, wurden in den späteren Druckausgaben des Pesttraktates von Matthias Böham medizinische Schaubilder mit expliziten Erörterungen der Lage und Eigenschaften der Blattern sowie Beulen veröffentlicht (Abb. 11) ${ }^{398}$.

Mit den ersten Maßnahmen zur Heilung der Beulen, auch Bubonen genannt, solle man nach der Ansicht der Augsburger Stadtärzte innerhalb von 24 Stunden nach Ausbruch der Krankheit beginnen, nachdem der Aderlass und die Schwitzkur durchgeführt wurden. Zunächst solle man die Schröpfköpfe direkt auf den Beulen ansetzen, um dadurch die in ihnen enthaltene verdorbene Materie herauszubefördern. Nachfolgend wurde die Schwellung mit einem Laßeisen angeritzt und hierauf erneut viele Schröpfköpfe nacheinander aufgesetzt, damit das Pestgift abfließen könne. Die Wunden seien anschließend mit speziell dafür zubereiteten Pflastern aus verschiedenen Kräutern und Heilstoffen zu umhüllen. Vor den Schmerzen bei dieser Behandlung solle man nicht zurückschrecken, so der Rat der Ärzte, weil das Gift auch dadurch wirksamer herausgezogen würde. Nach einigen Tagen seien die Beulen dann von einem Wundarzt aufzuschneiden und zu reinigen.

Die Blattern, die auch als Carbonen oder Antracem bezeichnet werden, sollen dagegen durchgestochen und dann mit einem Glüheisen ausgebrannt werden. Dadurch würde der Geschwulst ihr Gift vollständig genommen. Falls aber der Kranke sich der Behandlung des Ausbrennens verweigere, solle man die Blattern ebenso wie die Beulen mit Pflastern behandeln und dann von Wundärzten reinigen lassen. Die Augsburger Stadtärzte bevorzugten diese Heilmaßnahme im Gegensatz zu einigen anderen Medizinern, die zur Behandlung der Blattern ätzende Mittel empfahlen.

Die Pestbeulen konnten aber im Notfall auch selbsttätig bzw. durch Laien geöffnet werden.

Der Augsburger Doktor Johannes Klainmüller wies nämlich in seiner Pestschrift darauf hin, dass die arme Bevölkerung, insbesondere auf dem Lande, nicht jederzeit einen Arzt zur Verfügung habe ${ }^{399}$. Aus diesem Grund stellte er weitere Heilmethoden für die Apostemata

\footnotetext{
397 OCCO/SAYLER/TIEFFENBACH/TALHAUSER, Was die Pestilentz an jr selbs sey [Augsburg: Philipp Ulhart 1535], S. F Iv.

${ }^{398}$ BÖHAM, Ain nutzliche/ vnnd für den gemainen Man/ genugsam gegründte [Augsburg: gedruckt von Philipp Ulhart und zum Neudruck aufgelegt von Johannes Vogt dem Jüngeren um 1570; bearbeitet und ergänzt von Wolfgang Talhauser um 1547], auf dem Titelblatt: Die zway nachvolgende verzaichnete Bilder/ werden anzaigen durch die beygesetzten buchstaben/ wa unnd an wellichem ort des leibs/ hinden unnd vornen/ gewonlich die Apostemata herauß brechen/sampt etlichen boesen Blatern. Dazu siehe S. 68-70.

${ }^{399}$ KLAINMÜLLER, Ain hymlischen vnn Natürlichen anzaygung [Augsburg: Hans Schönsberger, 12. Oktober 1521].
} 
dar, die auch ohne ärztliche Hilfe ausgeführt werden konnten. Mit der Anwendung der von ihm beschriebenen Pflaster können die Beulen innerhalb von 24 Stunden geöffnet werden. Wenn diese nicht von allein aufbrechen, sollte man durch die Beule einen Wollfaden ziehen und für einige Tage darin stecken lassen. Klainmüller riet dazu die Apostemata mit Salben u.ä. Linderung verschaffenden Mitteln weiter $\mathrm{zu}$ behandeln, denn um eine möglichst vollständige Ausscheidung der Giftstoffe zu gewährleisten, durfen die Wunden nicht zu schnell zuwachsen. Die Vermögenden, die vill pfenning haben, sollen sich jedoch von erfahrenen Doktoren und Wundärzten behandeln lassen ${ }^{400}$.

Nach der Durchführung der besprochenen Behandlungen sei die Therapie des Kranken keinesfalls zu unterbrechen, sondern auch während der nächsten Tage mit allen Mitteln fortzusetzen, um das Gift zur Gänze aus dem Körper zu treiben ${ }^{401}$. Während dessen können dem Kranken Arzneimittel für die Stärkung des Herzens gereicht werden und auch die Anwendung des Klistiers und der Schwitzkur können wiederholt werden. Die speziellen Maßregeln der Diätetik waren weiterhin streng zu beachten, wonach der Kranke vor allem mit Hühnerbrühe und ähnlichen kräftigenden Speisen unter Zusatz von Essig, wie auch mit viel Gersten- und Sauerampferwasser, ernährt werden solle.

Darüber hinaus wurden in den Pestschriften weitere Anweisungen zur richtigen Krankenpflege aufgestellt. Der Pestkranke sei nämlich in einer separaten, nicht zu warmen Kammer unterzubringen, in der ein Luftzug herrsche und deren Fenster nach Norden gerichtet sein müssen. Wenn es möglich sei, solle man den Kranken mehrmals am Tag in eine andere Kammer verlegen und inzwischen in der leeren Stube die verdorbene Luft mit Feuer reinigen. Zu diesem Zweck solle man Räucherwerk und wohlriechende Kräuter gebrauchen. Darüber hinaus seien die Stirn, die Schläfen, die Hände des Kranken oder der Bereich unter seiner Nase mit Rosen- oder Lavendelwasser unter Zugabe von Essig einzureiben oder ihm damit getränkte Schwämme zum Riechen zu geben. Zur äußerlichen Anwendung könne man auch auf die Brust des Kranken ein weiches Tuch mit wohlriechendem Wasser legen. Zusätzlich seien Edelsteine von großem Nutzen, die mit ihren Eigenschaften das Herz zusätzlich kräftigten. Es sei gleichermaßen ratsam, den Kranken in vielfältiger Art und Weise

\footnotetext{
${ }^{400}$ Ebd., S. B IVv-C I.

401 Darauf weisen die Augsburger Stadtärzte hin, die in dieser Hinsicht viele Missstände beobachten, OCCO/SAYLER/TIEFFENBACH/TALHAUSER, Was die Pestilentz an jr selbs sey [Augsburg: Philipp Ulhart 1535], S. E IIIv: wir sehen ja in täglicher erfarung/das vilen leichtlich geholffen wurde/ so sy beharreten unnd nit gleich nach der lässe und schwaiß trunck nach liessen radt zubrauchen und zuvolgen/ dann der mehrerthail so er sich ain wenig wol befindt/ so tröst er sich selbs gar zu wol/ ach sagen sy/ es ist nichts gewest/ es ist mir nur ain klains beützlin aufgefaren/das thut mir auch nit weh/ ja es wäre nützer der beützel wer gro $\beta$ und that dir weh/ dann der wehtagen zeücht das gifft an sich au $\beta$ dem leib/ wir sehen in dieser Pestilentz vil die mit aim klainem beützelin drey/vier/ ja biß in acht tag umbgend/ und auff die lest so fört es erst herfür mit schmertzen und grösse.
} 
aufzumuntern und ihm ebenso religiösen Trost und Beistand zu gewähren, weil die Krankheit bei einem heiteren Gemützustand bedeutend schneller überwunden werden könnte.

In den Pestschriften werden auch spezielle Ratschläge für die Krankenpfleger aufgestellt, die on zweifel durch die gnad des allmechtigen gots/fürsehen werden/doch so vil natürlich/ sollen sy sich auch deren ding gebrauchen/ die solchen ungesund/ so von den krancken gat/ in dest minder schaden müg ${ }^{402}$. Die Aufwärter sollen demnach morgens Prophylaktika wie z.B. Theriak mit Wein einnehmen und andere vorbeugende Mittel wie z.B. eine die Luft reinigende Wurzel im Mund oder aromatische Kräuter bei sich tragen. Mehrmals täglich sollen sie ihre Hände und ihr Gesicht mit Rosenwasser oder gesalzenem Wasser mit Essig vermischt abwaschen. Besonders müssen sie sich vor dem giftigen Atem und den körperlichen Ausscheidungen der Kranken mit Hilfe von Räucherwerk und der Einhaltung von Hygienemaßnahmen schützen. Darüber hinaus sollen die Pfleger häufig die Krankenkammer verlassen, um gesunde Luft aus dem Norden einzuatmen.

\section{Wirksamkeit der medizinischen Pestlehre}

Zum Schluss gilt es noch die Frage zu besprechen, wie die Pestlehre von den Medizinern selbst eingeschätzt wurde. Nach der Analyse der Pestschriften lässt sich generell feststellen, dass die Pest damals durchaus als die gefährlichste Krankheit wahrgenommen wurde, bei der jedoch eine erfolgreiche Bewältigung weitgehend möglich war. Die Doktoren waren sich dennoch bewusst, dass auch bei gezielter Anwendung aller ihnen zur Verfügung stehenden Heilverfahren nicht immer die höchste Wirksamkeit der medizinischen Therapie erreicht werden könnte: alle geleert der ertzney/ kain gewisse fürsehung unnd hillf darfür erfunden haben/ bis auff diese zeit ${ }^{403}$. Ihrer Erfahrung nach konnte man häufiger den Tod als die Genesung der Kranken erwarten, gerade wenn die Behandlung zu spät begonnen wurde. Auch beim Erscheinen der Pestbeulen auf den bestimmten Stellen des Körpers musste man eher damit rechnen, dass diese menschen bleiben selten lebendig oder sy sterben gemainklich auf den vierzehenden $\operatorname{tag}^{404}$. Wenn aber die Therapie der Kranken rechtzeitig und ordnungsgemäß durchgeführt würde, könnten dank der Pestlehre ansehnliche Resultate erreicht werden. Die Augsburger Stadtärzte betonten in diesem Kontext eine relativ niedrige Sterblichkeitsrate im

\footnotetext{
${ }^{402}$ JUNG, Ain nutzliche trostliche und kurze underrichtung (1) [Augsburg: Silvan Ottmar 1521], S. B VI.

${ }^{403}$ Ebd. S. A II.

${ }^{404}$ BÖHAM, Ain nutzliche/ vnnd für den gemainen Man/ genugsam gegründte [Augsburg: gedruckt von Philipp Ulhart und zum Neudruck aufgelegt von Johannes Vogt dem Jüngeren um 1570; bearbeitet und ergänzt von Wolfgang Talhauser um 1547], S. D II.
} 
städtischen Pesthaus, das bezüglich der medizinischen Behandlung unter ihrer Aufsicht stand: es ist diese vergifftung so bey uns ist/ nit als gar schwär wann man im recht thut/ es ist ye ain gnädige straff/ das unter ainer solchen mänig volck/ so wenig im Pestilentz hauß/ ettwan ain wuchen au $\beta$ hundert und $x l v i i$ nur ix etwan $x v$ oder xvi sterben.(...) es geht gleich wol mit aim schneller zu dann mit dem andern/ dann die feüchtigkait der cörper seind ungleich ${ }^{405}$. Die Doktoren waren davon überzeugt, dass vor allem die korrekte Umsetzung und Anwendung der Pestlehre und die unermüdliche Krankenpflege entscheidend für die wirksame Bekämpfung der Pestepidemien sei. Die Genesung eines Pestkranken hinge aber immer auch von der Gnade Gottes ab, denn Gott allein sei allmächtig und nur er könne endgültig über Leben und Tod der Menschen bestimmen: Gott der Herr wölle durch Christum unsern Herren und genädig und barmhertzig sein/ diese mittel durch uns anzaigt/ wa es raicht zu erweyterung seines hailigen reychs/ fruchtbar machen/ oder diese erschrockliche kranckhait von uns/ und sein götliche gnad zu uns wenden/damit wir nit in versuchung gefürt sonder von allem übel erlöset werden ${ }^{406}$.

Die zeitgenössische Vorstellung, dass die Wirkungskraft der medizinischen Maßnahmen vom göttlichen Willen bedingt wurde, ist keinesfalls als Ausdruck der Ratlosigkeit der Mediziner gegenüber der schwer heilbaren Krankheit $\mathrm{zu}$ interpretieren, sondern ergibt sich logischerweise aus den Grundsätzen der Pestlehre. Nach der damaligen Ätiologie war die primäre Ursache der Pest immer eine Heimsuchung Gottes, sie konnte zugleich nur mit seiner Gnade abgewendet werden. Darin tritt noch einmal das gewichtigste Merkmal der vormodernen Medizinlehre zutage, deren Grundlagen vom religiösen Diskurs der Zeit geprägt waren.

\footnotetext{
405 OCCO/SAYler/TiEfFEnBACh/TALHAUSER, Was die Pestilentz an jr selbs sey [Augsburg: Philipp Ulhart 1535], S. E IIIv.

${ }^{406}$ Ebd. S. F III; Zu diesem religiösen Denkmuster in der Pestlehre auch z.B. in JUNG, Ain nutzliche trostliche und kurze underrichtung (1) [Augsburg: Silvan Ottmar 1521], S. B VI: und so solches alles so ordenlich gebraucht wirt/ bin ich one zweyfel/ der allmechtig ewig got/ wird uns allen mit seinen hilff/ gnedigklich erspriessen zu aller gesunthait.
} 


\section{Anfänge der städtischen Pestpolitik in Augsburg zwischen 1494 und 1521}

Im spätmittelalterlichen Augsburg war die städtische Pestbewältigung grundlegend vom religiösen Diskurs geprägt, weshalb die diesbezüglichen Praktiken vor allem von den kirchlichen Institutionen geleitet wurden ${ }^{407}$. Obrigkeitliche Maßnahmen des Rates selbst lassen sich dagegen lediglich bei der Organisation des Begräbniswesens in der Pestzeit nachweisen. Dabei richteten sich die Verordnungen des Rates erkennbar nicht nach den Grundsätzen der damaligen Pestlehre. Die Pestopfer wurden nämlich auf einigen innerstädtischen Kirchhöfen, darunter auch direkt im Stadtzentrum an der Domkirche begraben. Auch die Leichen der auswärts verstorbenen Bürger konnten damals aus den Nachbarorten wieder nach Augsburg zur Bestattung zurückgebracht werden. Derartige Begräbnispraktiken in der Stadt weisen auf die geringe Rolle der medizinischen Pestlehre hin, deren prophylaktische Anweisungen ausdrücklich zum Schutz vor Gestank und Ausdünstungen aus Gräbern oder unbegrabenen Körpern aufforderten ${ }^{408}$. Aufgrund der damaligen Entwicklung des Medizinalwesens ist jedoch vorauszusetzen, dass von einer gewissen Menge der Zeitgenossen während der Epidemien die entsprechenden medizinischen Maßnahmen ergriffen werden konnten. Im Hinblick auf die vorgenannten Handlungen des Rates ist jedoch anzunehmen, dass die medizinische Bewältigung der Pest zu jener Zeit nicht obrigkeitlich geführt wurde, sondern lediglich auf privater Ebene erfolgte. Eine städtische Pestpolitik, deren Grundlagen sich direkt auf den medizinischen Diskurs bezogen, kann man dagegen erst am Ende des 15. Jahrhunderts eindeutig belegen.

\section{Anlegung des Unteren Gottesackers $1494 / 1495$}

Im Sommer 1494 regierte die Pest erneut in Augsburg ${ }^{409}$. Im Gegensatz zu früheren Pestepidemien wurden dieses Mal vom Rat die ersten Verordnungen erlassen, welche den medizinischen Grundsätzen entsprachen. Diese mussten allerdings mit den kirchlichen Machtträgern in Augsburg vereinbart werden. In einer am 26. November 1494 ausgestellten Urkunde bestätigte der Augsburger Bischof Friedrich Graf von Zollern einen Vergleich

\footnotetext{
${ }^{407}$ Siehe S. 30-42.

${ }^{408}$ Siehe hierzu den Teil über den Schutz vor der verdorbenen Luft, S. 97-100.

${ }^{409}$ StChr, Bd. 23, S. 65.
} 
zwischen dem Domkapitel mit Dekan Ulrich von Rechenberg sowie dem Rat und den Bürgermeistern über die Einrichtung eines neuen Friedhofes ${ }^{410}$. Da die Kirchhöfe der beiden Augsburger Pfarren St. Moritz sowie die der Domkirche überfüllt waren, verständigte man sich darauf, während der jetzigen Epidemie an einem entlegenen Ort in der Stadt einen Gottesacker anzulegen ${ }^{411}$. Zu diesem Zweck wurden bald vier Gärten im Lugensland hinter der St. Stephan Kirche am nördlichen Rande der Stadt bei den Stadtmauern gelegen, vom Rat angekauft $^{412}$. Die Anlegung dieses Gottesackers (anfänglich St. Stephan Gottesacker genannt und im 16. Jahrhundert vor allem als Unterer Gottesacker bezeichnet) wurde Anfang 1495 vollzogen, so dass am 18. Februar Hans Hainhofer, Tuchscherer, als der erste Verstorbene dort begraben werden konnte. Am 2. April 1497 konnte auch die dort erbaute Salvatorkirche eingeweiht werden, deren Bau jedoch erst 1499 vollendet wurde ${ }^{413}$. Der eingerichtete Gottesacker sollte dabei sowohl während der gegenwärtigen als auch in künftigen „,gemeinen tods oder Pestilentz leuffen“ wie auch ,zu gesundtlichen Zeiten“ benutzt werden ${ }^{414}$. In dieser bischöflichen Urkunde zählte man auch alle Zielgruppen auf, die auf diesem neuen Friedhof ihre letzte Ruhestätte finden sollten: dienennd Ehallten bey Gaistlichen und Weltlichen dieser Statt herkomen Person, Billgrin und Geste, auch Burger und Inwoner, und all ander die aus Notturft oder Andacht darin zu ligen begern wurden ${ }^{415}$. Daraus kann man schließen, dass der neu angelegte Gottesacker zum Zeitpunkt seiner Gründung für die Bestattung der Armen vorgesehen war, die sich kein eigenes Grab leisten konnten. Solche Personen wurden während der früheren Pestepidemien in Massengräbern auf den innerstädtischen Kirchhöfen begraben ${ }^{416}$. Durch die Anlage dieses städtischen Gottesackers wollte der Rat vor allem die massenhaften Begräbnisse der Armen im Stadtzentrum abschaffen und an einem abgelegenen Ort in der Stadt verlegen. Ein dazu verordneter Platz lag zwar innerhalb der Stadtmauer, befand sich aber deutlich am Rande der Stadt. Auf dem Stadtplan um 1550 aus der „Cosmographia“ von Sebastian Münster wurde dieser weit vom Stadtzentrum entfernte Gottesacker auf der rechten Seite des Bildes anschaulich markiert (Abb. 17).

\footnotetext{
${ }^{410}$ SStBA, $2^{\circ}$ Cod. Aug. 214, T. III, Supl. T. II., 1494: Vergleich zwischen dem Domkapitul und der Stadt Augspurg, die Aufrichtung eines neuen Gottesackers betreffend.

${ }^{411}$ Der Friedhof an der Domkirche sollte laut dem Vergleich jetzt nicht mehr benutzt und dann nach einigen Jahren endgültig abgetragen werden.

${ }^{412}$ SStBA, $4^{\circ}$ Cod. Aug. 62: Vorrede zu diesem Band; SStBA, $2^{\circ}$ Cod. Aug. 80, Fol. 48; StChr, Bd. 23 , S. 66.

${ }^{413}$ Auf Initiative des Rates gaben zunächst die städtische und kirchliche Partei je 200 Gulden für den Bau der Kapelle auf dem Friedhof, die dann aus den einfließenden Opfergeldern zurückbezahlt werden sollten. Im Jahr 1499 wurden zu diesem Zweck nochmals je 300 Gulden unter den gleichen Bedingungen eingereicht, in: KIEßLING, Bürgerliche Gesellschaft, S. 128-129.

${ }^{414}$ SStBA, $2^{\circ}$ Cod. Aug. 214, T. III, Supl. T. II., 1494.

415 Ebd.

${ }^{416}$ Siehe S. 36.
} 


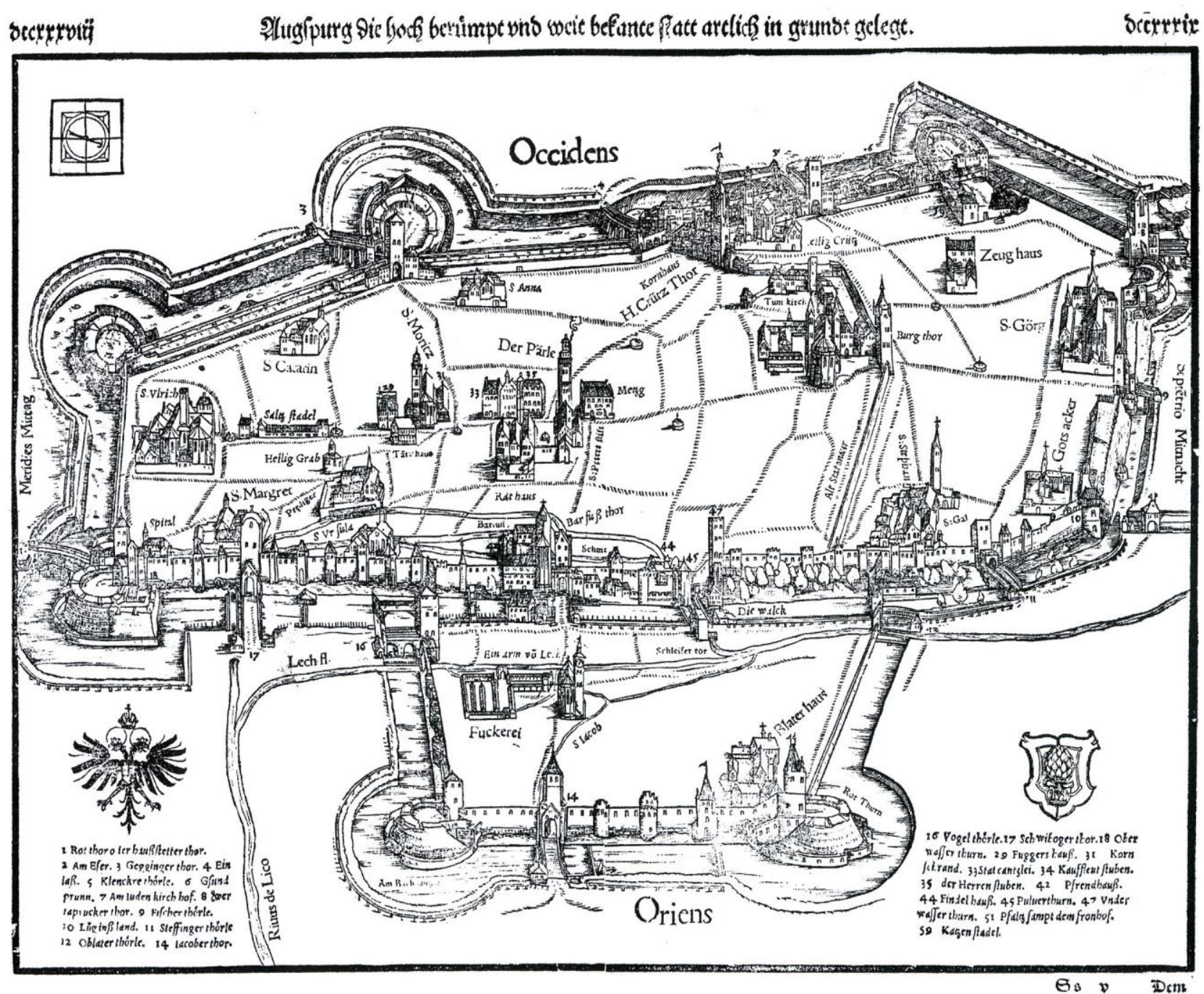

Abb. 17: Augsburger Stadtplan um 1550, in: Sebastian Münster, Cosmographia, ca. 1550, Städtische Kunstsammlungen Augsburg

Obwohl zu diesem Zeitpunkt noch nicht alle Begräbnisse in der Pestzeit an dieses Randgebiet Augsburgs verlegt worden waren, kann man diese Verordnung als einen Ausgangspunkt städtischer Maßnahmen ansehen, mit denen man den städtischen Raum vor der Verunreinigung durch schädliche Ausdünstungen aus den überfüllten Gräbern schützen wollte. 


\section{Einrichtung der Pesthäuser 1494}

Die zweite Verordnung des Rates während der Pestepidemie 1494 betraf die Einrichtung spezieller Häuser für die Armen unter den Pestkranken. In der bischöflichen Urkunde wurde nämlich vermerkt, dass ein vom Rat angestellter Priester die Krancken so in die hewser die in disen sorgfelltigen fürfallenden tods Leuffen dartzu verordent und bestellt seyen getragen und gefürt mit den heiligen Sakramenten versehen solle ${ }^{417}$. Über die erwähnten Häuser finden sich in diesem Dokument keine weiteren Angaben. In den Baumeisterrechnungen wurde dagegen am 22. November 1494 der Ankauf eines großen Hauses für die Pestkranken verzeichnet, das die Stadt für die beachtliche Summe von 306 Gulden von dem Ritter Caspar Stunz erworben hatte $^{418}$. Nach dem Ende dieser Pestepidemie wurde das Haus jedoch schon 1495 für die Unterbringung derjenigen Kranken gebraucht, die von einer als „Franzosenkrankheit“, „Franzosenpocken“, „Morbus Gallicus“ oder „Blattern“ genannten Seuchenkrankheit betroffen waren ${ }^{419}$. Die rasche Verbreitung dieser neuen Krankheit in Augsburg veranlasste den Rat offensichtlich dazu, das leer stehende Anwesen in das städtische Blatterhaus für die Franzosenkranken umzugestalten. Dieses zweistöckige Gebäude lag direkt bei den äußeren Stadtmauern, ganz am Rande der Jakobervorstadt im Augsburger Armenviertel. Seine Lage am Roten Turm in der Nähe des Jakobertores war weit vom Stadtzentrum entfernt und dadurch auch für die Isolierung der dort untergebrachten Pest- und Franzosenkranken geeignet (Abb. 17). Laut den Schilderungen in den zeitgenössischen Chroniken wurde das Blatterhaus, in dem gleichzeitig bis zu 125 Personen untergebracht werden konnten, auch in den danach folgenden Pestepidemien zur Unterbringung der erkrankten Armen und Dienstleute benutzt: und hatten die von Augsburg ein Haus gemacht, wann es hie starb an der Pestilentz, so legt man arme Leute und eehalten darein, und in dem selben Haus auf anthieltt man das arm folk mit dem plattern, der sind woll auf einmal 125 menschen darin gewesen, gab man umb gottz willen zu essen und zu loben Gottes ${ }^{420}$. Die institutionelle Fürsorge von beiden Krankengruppen fand in diesem Gebäude aber nur bis zum Jahre 1521 statt, als der Rat ein spezielles Pesthospital außerhalb der Stadtmauern Augsburgs gegründet hatte.

\footnotetext{
417 SStBA, $2^{\circ}$ Cod. Aug. 214, T. III, Supl. T. II., 1494: Vergleich zwischen dem Domkapitul und der Stadt Augspurg, die Aufrichtung eines neuen Gottesackers betreffend.

418 StAA, Baumeisterrechnungen, 1494, Samstag vor Katharine (22. November): „it. 306 guldin umb des Stuntzen hawß beym rotten turn und ganntze hofraitin, darein man die lewt legen wurdet, die den geprechen haben".

${ }^{419}$ In der bisherigen Forschung wurde meistens die frühneuzeitliche Franzosenkrankheit mit dem modernen Krankheitsbild der venerischen Syphilis gleichgesetzt, was sich jedoch wegen der fragwürdigen Grundannahme der retrospektiven Diagnose nicht mehr kritiklos halten lässt, dazu in: STEIN, Franzosenkrankheit, S. 11-28.

${ }^{420}$ SStBA, $2^{\circ}$ Cod. Aug. 71, Fol. 188; StChr, Bd. 23, S. 422.
} 
Anhand der Überlieferung aus späterer Zeit kann man zugleich annehmen, dass während der Pestepidemien zwischen 1494 bis zum Jahr 1521 noch ein zweites Haus für die Pestkranken in der Jakobervorstadt eingerichtet wurde. Im Jahr 1563 versorgte man die ersten Kranken während der damaligen Pestepidemie zunächst im kleinen sog. ,inneren“ Pesthaus, das sich in der nächsten Umgebung des 1494 angekauften Anwesens befand ${ }^{421}$. In diesem kleinen Gebäude konnten aber nur bis zu 30 Personen untergebracht werden. Dass man in diesen zwei benachbarten Häusern in der Jakobervorstadt ursprünglich die städtische Pestfürsorge organisierte, bezeugt noch ein anderes Dokument. In dem Bittschreiben des Rates an Papst Leo X. (1513-1521) um eine Bulle für das 1521 gegründete Pesthospital wurden auch die schon bestehenden Gebäude für die Pestkranken in Augsburg erwähnt ${ }^{422}$. Der Rat berichtete hier, dass in den vergangenen Jahren in der Vorstadt zwei Häuser, darunter eines für die Franzosenkranken, von der Stadt eingerichtet worden sei, in denen die mittellosen Kranken in der Pestzeit versorgt und von den Medizinern behandelt würden. Da aber die gegenwärtige Pestepidemie die Menschen in Augsburg stärker als zuvor befiele, reichen die dazu verordneten Häuser nicht mehr aus ${ }^{423}$. Aufgrund dieser Hinweise ist anzunehmen, dass die städtische Fürsorge für die Pestkranken am Ende des 15. Jahrhunderts vornehmlich in der Jakobervorstadt organisiert worden war. Sowohl die eingerichteten Pesthäuser wie auch der im Jahre 1494 angelegte Gottesacker waren dabei vor allem für die Versorgung der Armen in der Stadt vorgesehen ${ }^{424}$.

Die Gründung der neuen Friedhofsanlage und der Pesthäuser erforderte eine Vereinbarung mit dem Bischof, dem Domkapitel und allen Pfarr- und Kirchherren in Augsburg, weil einige Fragen der Seelsorge bei den Begräbnissen und den daraus fließenden

\footnotetext{
${ }^{421}$ StChr, Bd. 32, S. 486; Auf dem Stadtplan Augsburgs im 19. Jahrhundert waren das sogenannte ,innere“ Pesthaus als H 290 und das Blatterhaus als H 282 bis 285 vermerkt.

${ }^{422}$ SStBA, $2^{\circ}$ Cod. Aug. 215, T. IV, Supl. T. III., 1521: Des Raths zu Augspurg Bittschreiben um eine Bulle über die 2. Brechhäuser, an Pabst Leo X.

${ }^{423}$ Ebd.: Magistri civium et Senatores ab annis Superioribus instituerunt et erexerunt pro bono et commoditate reip. in suburbio eiusdem urbis duas domus contiguas, unam pro laborantoribus morbo Gallico et alteram qua tempore pestilentiae homines pauperes hoc morbo laborantes reciperentur, et ibi emolumenta et medicos haberent; easdem domus hactenus cum omnibus necessariis de pecunia reip. quam ut plurimum et maxime cives Augustani solitis tributis et nectigalibque pro debita impositione collatis sustentant. Verum cum iidem Magistri civium et Senatores hoc anno perspexisent, morbum pestilentia hunc ingruere, et plus quam superioribus annis et quod pradicta domus pro infirmis recipiendis non suficeret.

${ }^{424}$ In den noch vor dem Vergleich aus dem 26. November 1494 verfassten Aufzeichnungen des Rates über die Einrichtung des Gottesackers wurden die speziellen Häuser für die Pestkranken ursprünglich in jeder Pfarre geplant, falls die ausgebrochene Epidemie sich weiter in der Stadt verbreitet hätte: item ob die sterben leuff sich erheben unnd zunemen wurden das dann in ainer yden pfarre ain besonder haws bestellt darein Ehallten So bey gaystlichen und weltlichen krannck wurden und die das begeren oder annder kranncke not So es notturfftig were genomen unnd den darinn zu zewarten bestellt werden sol mit ainer ordnung wie dann die nach gut ansehen erfunden und gemacht wurd; in: StAA, CM IV, Nr. 3: Pläne des Rates zur Anlegung des Gottesackers (1494). Darüver, ob die Pesthäuser noch in anderen Orten in Augsburg eingerichtet wurden, sind keine Hinweise überliefert.
} 
Einkünften neu geregelt werden mussten ${ }^{425}$. In diesem Vergleich wurde von den weltlichen und kirchlichen Machtträgern allgemein festgelegt, dass die bisherigen Rechte aller Pfarren, von einigen gemeinsam vereinbarten Ausnahmen abgesehen, nicht beeinträchtigt werden sollen. Man einigte sich dahingehend, dass alle Gaben und Vermächtnisse für die neuen städtischen Einrichtungen - Gottesacker und Pesthäuser - nur an einen speziellen Opferstock fallen sollen, was anscheinend eine Verminderung der pfarrlichen Einnahmen bedeutete. Die Verwaltung dieser Opfergaben solle gemeinsam von der Kirche und der Stadt durchgeführt werden, die dazu je einen Verordneten aus dem Domkapitel und dem Rat ernannten. Das gesamte Opfergeld solle dann ausschließlich zum Bedarf des Gottesackers und der Pesthäuser oder zur künftigen Errichtung einer Kapelle auf dem Friedhofsgelände verwendet werden.

Darüber hinaus war die bischöfliche Erlaubnis dazu notwendig, weil der Rat für die Dauer der Pestepidemien einen Priester auf Widerruf anstellen wollte. Das Patronatsrecht über diese Priesterstelle wurde dem Rat erteilt, der seither in der Pestzeit einen Geistlichen, nach vorheriger Präsentation des Kandidaten vor einem Vikar, dort frei einsetzen und auswechseln konnte. Der dazu verordnete Priester sollte speziell für die Seelsorge der Kranken in den eingerichteten Pesthäusern zuständig sein. Er wurde auch verpflichtet, die Namen aller auf dem Gottesacker begrabenen Personen den Pfarreien in Augsburg schriftlich bekannt zu geben, damit man den Verstorbenen gemeinsam oder individuell während der Predigten gedenken und für sie die Totenmesse abhalten könne. Für all jene Verstorbenen, die auf dem Gottesacker begraben, aber denen wegen ihrer Armut in ihren angestammten Pfarreien nicht im Besonderen gedacht werden könne, solle dieser Priester einmal in der Woche eine Messe zu ihrem Seelenheil lesen ${ }^{426}$.

\footnotetext{
${ }^{425}$ SStBA, $2^{\circ}$ Cod. Aug. 214, T. III, Supl. T. II., 1494. Dazu Braun, Bischöfe, Bd. 3, S. 142-143.

${ }^{426}$ Bevor der Vergleich mit den kirchlichen Würdenträgern abgemacht wurde, plante der Rat ursprünglich die Seelsorge in der Pestzeit auf eine andere Art und Weise zu organisieren. Falls die Epidemie sich weiter verbreitet hätte und die Pesthäuser für die armen Dienstleute in allen Pfarren einzurichten gewesen wären, sollten die Pfarrer oder ihre Helfer die Seelsorge der Kranken übernehmen. Ungeachtet der Tatsache, ob man die Verstorbenen aus demselben Sprengel oder aus dessen anderen Stadtvierteln in die Pesthäusern verordnete, sollten die Pfarrer für sie die Totenmessen lesen, wenn von ihnen dafür ausreichendes Hab und Gut hinterlassen wurde. Den unvermögenden Dienstleuten sollte dagegen in den Pfarrkirchen gedacht werden, in denen ihre Dienstherren Messen zu diesem Zweck gestiftet hätten. In: StAA, CM IV, Nr. 3: Pläne des Rates zur Anlegung des Gottesackers (1494).
} 


\section{Die Pestepidemien von $1504 / 05$ und $1511 / 12$}

Das Auftreten der nächsten Pestepidemien in den Jahren 1504/05 und 1511/12 kann man einzig anhand der sogenannten Augsburger Bevölkerungstafel nachweisen. Dieses Geburts-, Hochzeits- und Sterberegister wurde jährlich von 1501 bis in die zweite Hälfte des 18. Jahrhunderts hinein aufgrund der wöchentlichen Meldungen der Pfarreien an den Rat zusammengestellt ${ }^{427}$. Diese vitalstatistischen Daten liegen dabei sowohl in handschriftlicher Form in einem der Augsburger Hochzeitsbücher wie auch in mehrmals aufgelegten Einblattdrucken (Abb. 18 und 19) vor ${ }^{428}$. Die relativ hohe Zuverlässigkeit der Bevölkerungstafel veranlasste die meisten Forscher ihre Studien zur demographischen Entwicklung Augsburgs in der Frühen Neuzeit gerade auf diese ungewöhnliche Quelle zu stützen. Im Hinblick auf die Erforschung der Pestepidemien sind diese zeitgenössischen Statistiken ebenfalls von großer Bedeutung, weil in den Einblattdrucken aus dem 17. und 18. Jahrhundert mit einem Kreuzzeichen vermerkt wurden, wann ein sterbend gewesen. Die darunter angegebenen Sterbezahlen lassen sich dabei mit den vorhergehenden und nachfolgenden Jahren vergleichen, wodurch es möglich ist, die demographischen Verluste in der Pestzeit allgemein zu berechnen. Die in der Tafel angeführten Pestepidemien lassen sich dagegen mehrheitlich auch anhand der anderen zeitgenössischen Quellen bestätigen. Die einzigen Ausnahmen bilden hier die Seuchen von 1504/05 und 1511/12, für die leider keine anderen Quellenbelege gefunden werden konnten. Unter diesen Jahren wurden Todeszahlen von 4.765 und 3.564 Menschen für 1504/05 und von 4.870 und 2.980 Personen für 1511/12 verzeichnet. In den vorhergehenden und nachfolgenden Jahren waren dagegen die Sterberaten zwei bis drei Mal niedriger ${ }^{429}$. Aufgrund der hohen Todeszahlen, wie auch der Korrektheit der Bevölkerungstafel im Fall der anderen dort verzeichneten Pestjahre, kann man den tatsächlichen Ausbruch der Pest auch in dieser Zeitperiode als gegeben annehmen. Im Jahr 1505 kam aber in Augsburg noch eine andere Krankheit auf, die die damals ausgebrochene Pestepidemie zusätzlich begleitete.

\footnotetext{
${ }^{427}$ Zur Bedeutung dieser Quelle und zur Demographieforschung im frühneuzeitlichen Augsburg, in: JAHN, Einwohnerzahl; RAJKAY, Bevölkerungsentwicklung.

${ }^{428}$ Augsburger Bevölkerungstafel Nr. 142 (1501-1679), 143 (1501-1744), 144 (1501-1771), 347 (1511-1584), SStBA, $2^{\circ}$ Lw Einblattdrucke nach 1500. Die einzelnen Jahresangaben des Geburts-, Hochzeits- und Sterberegisters mit den graphischen Darstellungen der Bevölkerungsbewegung im 16. Jahrhundert wurden zusammengestellt in: JAHN, Einwohnerzahl, S. 386-391; Diese demographischen Graphiken werden auch präsentiert in: RAJKAY, Bevölkerungsentwicklung, S. 253. Die gedruckte Bevölkerungstafel mit den Angaben für die Jahre 1501-1750 wurde abgebildet, in: FISCHER, Gesundheitswesen, Bd. I, S. 67, Abb. 6.

${ }^{429}$ In der Bevölkerungstafel finden sich die folgenden Todeszahlen für die pestfreien Zeiten: 1503: 1.646, 1506 : 1.950, 1510: 1.979 und 1513: 1.960 .
} 


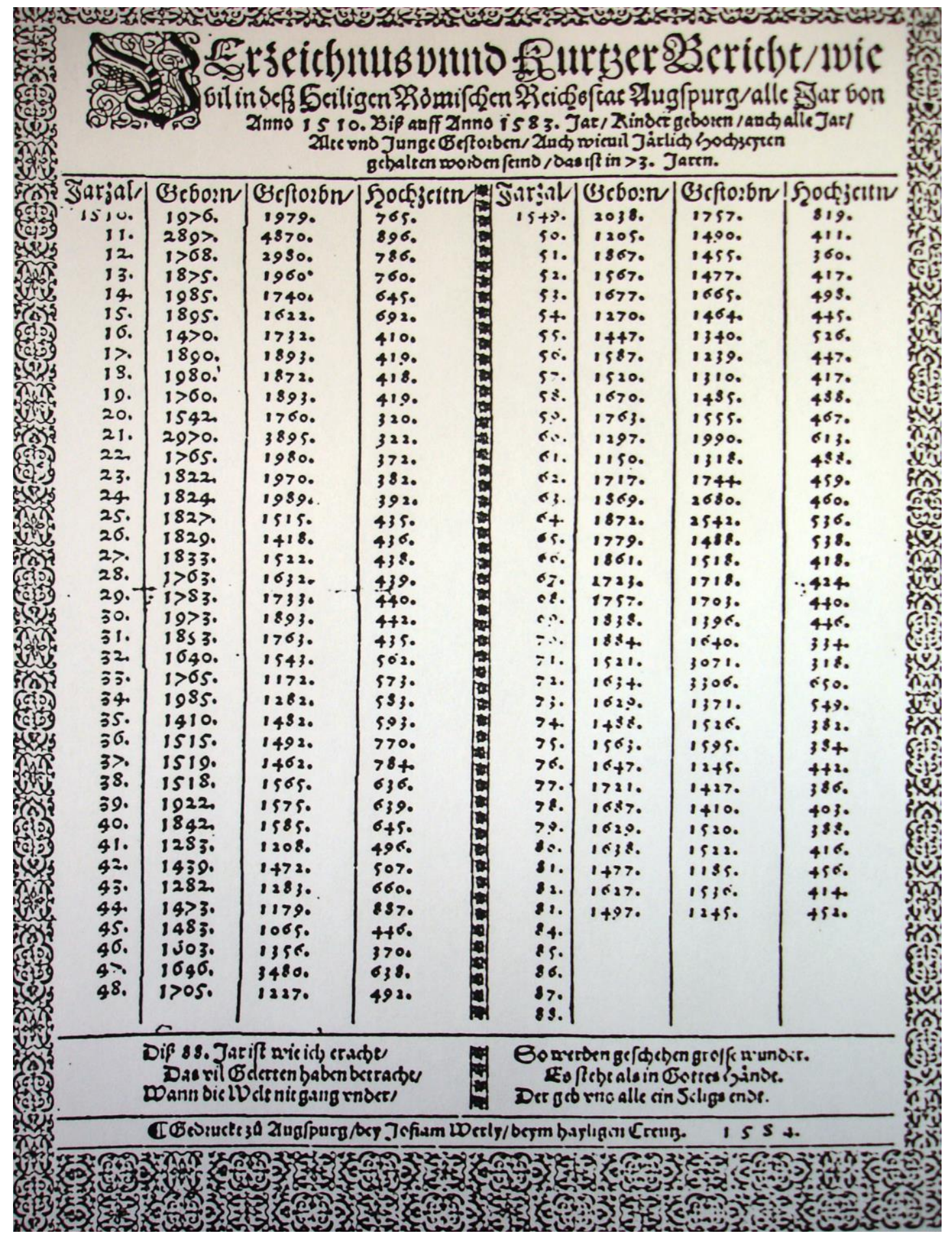

Abb. 18: Bevölkerungstafel (1510-1583), SSBA, $2^{\circ}$ Lw Einblattdrucke nach 1500, Nr. 347 (1511-1584) 


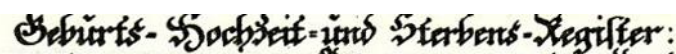

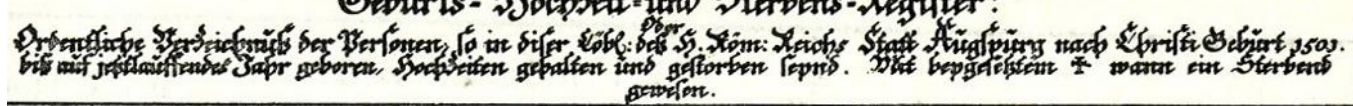

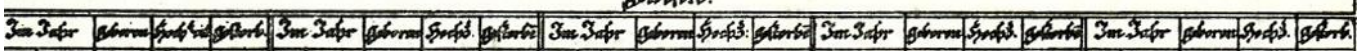

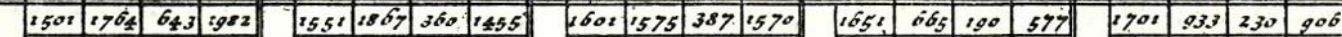

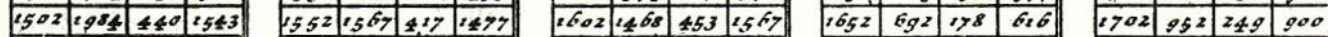

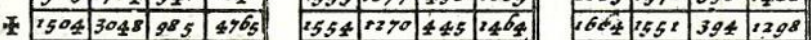

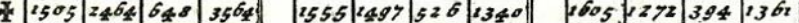

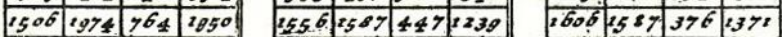

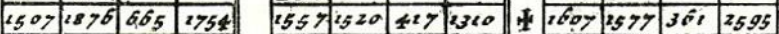
2508 2764 444284

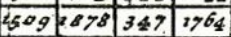
\begin{tabular}{ll|l|l|l|l|l}
\hline 510 & 2976 & 765 & 1999 \\
\hline
\end{tabular}

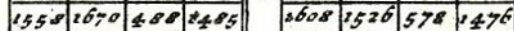
\begin{tabular}{|l|l|l|l|l|l|}
\hline 4559 & 1763 & 467 & 2553 \\
\hline 1600 & 1648 & 477 & 1469 \\
\hline
\end{tabular}

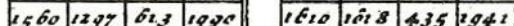
\begin{tabular}{|l|l|l|l|l|l|l|}
\hline 2511 & 2899 & 896 & 4870 \\
\hline 2562 & 1150 & 486 & 2310 \\
\hline
\end{tabular} \begin{tabular}{|l|l|l|l|l|}
\hline 1512 & 1968 & 486 & 2980 \\
\hline
\end{tabular}$\quad$\begin{tabular}{|l|l|l|l|}
\hline 2562 & 2717 & 454 & 1744 \\
\hline
\end{tabular}

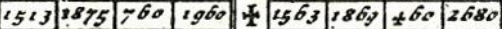

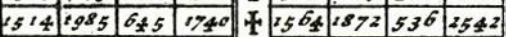
\begin{tabular}{|l|l|l|l|l|l|l|l|l|l|}
\hline 1555 & 1895 & 692 & 1622 \\
\hline 2565 & 1779 & 538 & 1488 \\
\hline
\end{tabular}

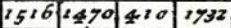

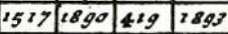
\begin{tabular}{|l|l|l|l|l|}
1528 & 2980 & 418 & 1872 \\
\hline
\end{tabular} \begin{tabular}{|l|l|l|l|}
\hline 1519 & 1260 & 419 & 1895 \\
\hline
\end{tabular} \begin{tabular}{ll|l|l|l|l|}
1566 & 1862 & 418 & 1518
\end{tabular} \begin{tabular}{|l|l|l|l|}
\hline 2567 & 1723 & 124 & 2788 \\
\hline
\end{tabular} \begin{tabular}{|l|l|l|l|l|l|}
2568 & 2757 & 440 & 2703
\end{tabular} \begin{tabular}{|l|l|l|l|}
\hline 2569 & 1838 & 446 & 2396 \\
\hline
\end{tabular} \begin{tabular}{|l|l|l|l|}
\hline 2520 & 1542 & 320 & 2700 \\
\hline
\end{tabular} \begin{tabular}{l|l|l|l|}
1570 & 7884 & 334 & 1640
\end{tabular}

\begin{tabular}{|l|l|l|l|}
\hline 1522 & 2970 & 322 & 3895 \\
\hline 1522 & 1765 & 372 & 1980
\end{tabular} \begin{tabular}{|l|l|l|l|}
\hline 1522 & 1765 & 372 & 1980 \\
\hline 1523 & 1828 & 382 & 1970 \\
\hline
\end{tabular} 25787521315.3071

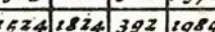
\begin{tabular}{|l|l|l|l|}
\hline 2525 & 1827 & 435 & 7515 \\
\hline
\end{tabular} \begin{tabular}{l|l|l|l|}
1526 & 1829 & 436 & 1428 \\
\hline 1527 & 1833 & 438 & 1522
\end{tabular} \begin{tabular}{|l|l|l|l|}
\hline 2527 & 2833 & 438 & 1322 \\
\hline 1528 & 12653 & 438 & 1633
\end{tabular} \begin{tabular}{l|l|l|l|}
1528 & 1763 & 439 & 1632 \\
\hline 1529 & 1783 & 440 & 1733
\end{tabular} \begin{tabular}{l|l|l|l|}
1529 & 1783 & 440 & 2733
\end{tabular} \begin{tabular}{l|l|l|l|l}
15.30 & 1973 & 442 & 1893
\end{tabular} \begin{tabular}{|l|l|l|l|}
\hline $153^{3}$ & 18.53 & 445 & 1763 \\
\hline
\end{tabular} \begin{tabular}{l|l|l|l|l}
$153^{2}$ & 1640 & 562 & 1543
\end{tabular} \begin{tabular}{l|l|l|l|l|l|}
\hline 1533 & 1765 & 573 & 1172 \\
\hline
\end{tabular} \begin{tabular}{ll|l|l|l}
1534 & 1985 & 583 & 1282
\end{tabular} \begin{tabular}{|l|l|l|l|}
\hline 2572 & 1634 & 650 & 3306 \\
\hline 2573 & 2539 & 549 & 1371 \\
\hline
\end{tabular}

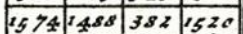
\begin{tabular}{|l|l|l|l|}
\hline 2575 & 1563 & 384 & 1595 \\
\hline
\end{tabular} \begin{tabular}{|l|l|l|l|}
\hline 2576 & 1647 & 442 & $12+5$ \\
\hline
\end{tabular}

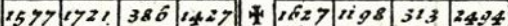

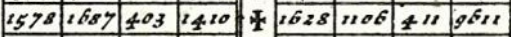

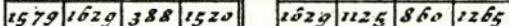

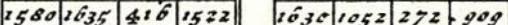
\begin{tabular}{|l|l|l|l|l|l|l|}
\hline 2582 & 2477 & 456 & $128_{5}$ \\
\hline 1631 & 1173 & 109 & 859 \\
\hline
\end{tabular} $\begin{array}{lllllllll}2582 & 1627 & 4 ! 2536 & 253\end{array}$

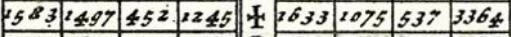

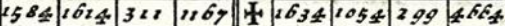

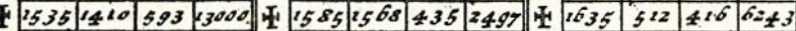

\begin{tabular}{|l|l|l|l|l|l|l|l|l|l|l|l|}
\hline 1536 & 2535 & 770 & 2492 \\
\hline 2586 & 2583 & 576 & 3136 \\
\hline 2636 & 789 & 440 & 700 \\
\hline
\end{tabular}

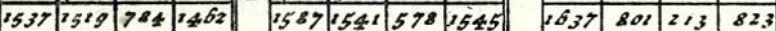
\begin{tabular}{|l|l|l|l|l|l|l|l|l|l|l|}
\hline 1539 & 1922 & 639 & 1575 \\
\hline 3589 & $268_{4}$ & 426 & 1372 \\
\hline
\end{tabular}

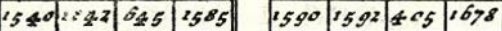

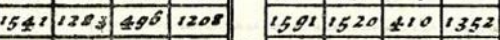

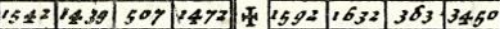

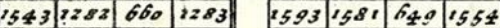

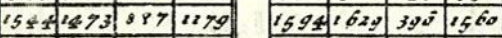

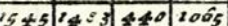
\begin{tabular}{|l|l|l|l}
1546 & 1603 & 370 & 1356 \\
\hline
\end{tabular}

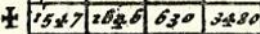

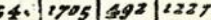
2549 20y? 939 175 \begin{tabular}{l|l|l|l|l|}
2595 & 1517 & 335 & 1584
\end{tabular} \begin{tabular}{ll|l|l|l|l}
2596 & 1039 & 4.37 & 2505 \\
\hline
\end{tabular} \begin{tabular}{|l|l|l|l|l|}
\hline 597 & 1608 & 393 & 1594 \\
\hline
\end{tabular} \begin{tabular}{l|l|l|l|l|}
2598 & 1552 & 380 & 1632 \\
\hline
\end{tabular} \begin{tabular}{|l|l|l|l|}
\hline 599 & 1480 & 386 & 1447 \\
\hline
\end{tabular} \begin{tabular}{|l|l|l|l|}
\hline 2600 & 1621 & 499 & 2775 \\
\hline
\end{tabular}

\begin{tabular}{|l|l|l|l|}
\hline 1653 & 690 & 150 & 575 \\
\hline
\end{tabular} \begin{tabular}{|l|l|l|l|}
\hline $165 x$ & 1944 & 197 & 764 \\
\hline
\end{tabular} \begin{tabular}{l|l|l|l|l|l|}
\hline 055 & 680 & 158 & 570 \\
\hline
\end{tabular}

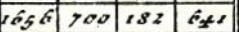
\begin{tabular}{|l|l|l|l|}
\hline 2657 & 675 & 182 & 730 \\
\hline 1658 & 659 & 198 & 750 \\
\hline
\end{tabular} \begin{tabular}{|l|l|l|l|}
\hline 1658 & 659 & 198 & $73^{1}$ \\
\hline
\end{tabular} \begin{tabular}{|l|l|l|l|}
\hline 1659 & 687 & 187 & 8.32 \\
\hline
\end{tabular} \begin{tabular}{l|l|l|l|}
1660 & 657 & 211 & 657
\end{tabular} \begin{tabular}{|l|l|l|l|}
\hline 668 & 710 & 179 & 668 \\
\hline
\end{tabular} \begin{tabular}{|l|l|l|l|}
\hline 1662 & 644 & 192 & $78 s$ \\
\hline
\end{tabular} \begin{tabular}{|l|l|l|l|}
1665 & 675 & 209 & 8.30 \\
\hline
\end{tabular}

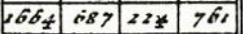

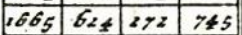
\begin{tabular}{l|l|l|l|}
2660 & 600 & 209 & 7.37
\end{tabular} \begin{tabular}{|l|l|l|l|}
\hline 3667 & 754 & 193 & 769 \\
\hline 1668 & 688 & 288 & 760 \\
\hline
\end{tabular} \begin{tabular}{l|l|l|l|}
1658 & fos & 228 & 721 \\
\hline
\end{tabular} \begin{tabular}{|l|l|l|l|}
\hline 1669 & 793 & 194 & $75 z$ \\
\hline
\end{tabular} \begin{tabular}{|l|l|l|l|}
\hline 1670 & 707 & 228 & $72 x$ \\
\hline
\end{tabular} \begin{tabular}{l|l|l|l|}
1672 & 810 & 210 & 728 \\
\hline
\end{tabular} \begin{tabular}{|l|l|l|l|}
\hline 1672 & 830 & $19 y$ & $786^{\prime}$ \\
\hline
\end{tabular} \begin{tabular}{|l|l|l|l|}
\hline 1673 & 786 & 239 & 751 \\
\hline 1674 & 874 & 230 & 842 \\
\hline
\end{tabular} \begin{tabular}{l|l|l|l|}
2674 & 874 & 231 & 842 \\
\hline
\end{tabular} \begin{tabular}{|l|l|l|l|}
\hline 2675 & 775 & 201 & 913 \\
\hline 1676 & 805 & 230 & 923 \\
\hline
\end{tabular} \begin{tabular}{ll|l|l|}
1676 & 805 & $23 \mathrm{C}$ & 913 \\
\hline 1679 & 845 & 228 & 930
\end{tabular} \begin{tabular}{lllll}
1677 & $s_{43}$ & 224 & 934 \\
\hline 678 & 854 & 230 & $g 43$
\end{tabular} \begin{tabular}{|l|l|l|l|}
\hline 2678 & 854 & 238 & 943 \\
\hline 6279 & 862 & 250 & 985 \\
\hline
\end{tabular} \begin{tabular}{l|l|l|l|}
1679 & 861 & 250 & 945 \\
\hline
\end{tabular} \begin{tabular}{l|l|l|l|l}
2680 & 807 & 281 & 946
\end{tabular} \begin{tabular}{|l|l|l|l|}
\hline 6681 & 888 & 214 & 860 \\
\hline
\end{tabular} \begin{tabular}{|l|l|l|l|}
\hline 1682 & 898 & 274 & 734 \\
\hline
\end{tabular} \begin{tabular}{|l|l|l|l|}
\hline $6 s s$ & $s g 2$ & 195 & $s 0 s$ \\
\hline
\end{tabular}

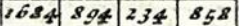
\begin{tabular}{l|l|l|l|}
\hline 1685 & 885 & 230 & 848 \\
\hline
\end{tabular} \begin{tabular}{|l|l|l|l|}
\hline 680 & 910 & 247 & 98. \\
\hline 1687 & 852 & 284 & 855 \\
\hline
\end{tabular} \begin{tabular}{l|l|l|l|}
\hline 1687 & 853 & 281 & 855 \\
\hline
\end{tabular} \begin{tabular}{l|l|l|l|}
\hline 2688 & 927 & 231 & $s 60$ \\
\hline
\end{tabular} \begin{tabular}{|l|l|l|l|}
\hline 1689 & 853 & 213 & 806 \\
\hline
\end{tabular} \begin{tabular}{l|l|l|l|l|l|l|l|}
1690 & 912 & 262 & 2078
\end{tabular} \begin{tabular}{|l|l|l|l|}
\hline 2692 & 325 & 253 & 785 \\
\hline 692 & 895 & 202 & 935 \\
\hline
\end{tabular} \begin{tabular}{l|l|l|l|}
1692 & 893 & 202 & $935^{\circ}$
\end{tabular} \begin{tabular}{|l|l|l|l|}
\hline 2693 & 818 & 219 & 2084 \\
\hline
\end{tabular} \begin{tabular}{|l|l|l|l|}
\hline 2694 & 760 & 247 & 2106 \\
\hline
\end{tabular} 2695 867 262 10.58 \begin{tabular}{l|l|l|l|}
2696 & 930 & 2.75 & $92 ;$
\end{tabular} \begin{tabular}{|l|l|l|l|}
\hline 2697 & 969 & 271 & 777 \\
\hline
\end{tabular} 1698 1020 $2 \neq 1 \quad s 70$ \begin{tabular}{ll|l|l|}
\hline 699 & 1008 & $z$ is & $9 \neq$
\end{tabular}

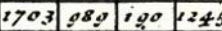
\begin{tabular}{|l|l|l|l|}
\hline 1404 & 818 & 339 & 3113 \\
\hline
\end{tabular} \begin{tabular}{|l|l|l|l|l|}
\hline 2405 & 890 & 405 & 748 \\
\hline
\end{tabular} \begin{tabular}{|l|l|l|l|}
\hline 1706 & 949 & 307 & 842 \\
\hline 707 & 2003 & 290 & 805 \\
\hline
\end{tabular}

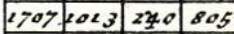
\begin{tabular}{|l|l|l|l|}
\hline 1708 & 920 & 225 & 908 \\
\hline
\end{tabular} \begin{tabular}{|l|l|l|l|}
\hline 1709 & 948 & 240 & 805 \\
\hline
\end{tabular} \begin{tabular}{|l|l|l|l|}
\hline 710 & 982 & 23 & 811 \\
\hline 1710 & 898 & 283 & $85 S$
\end{tabular} \begin{tabular}{|l|l|l|l|}
\hline 711 & 899 & 243 & 855 \\
\hline
\end{tabular} \begin{tabular}{|l|l|l|l|}
\hline 1712 & 911 & 229 & 894 \\
\hline
\end{tabular} \begin{tabular}{|l|l|l|l|}
\hline $17{ }^{\prime}$ & 837 & 202 & 860 \\
\hline
\end{tabular} \begin{tabular}{|l|l|l|l|}
\hline 2714 & 874 & 264 & 948 \\
\hline
\end{tabular} \begin{tabular}{|l|l|l|l|}
\hline 1715 & 866 & 309 & 1024 \\
\hline
\end{tabular} \begin{tabular}{|l|l|l|l|}
\hline 1716 & 997 & 272 & 905 \\
\hline
\end{tabular} \begin{tabular}{|l|l|l|l|}
\hline 1717 & $9^{2} 5$ & 250 & $9^{88}$ \\
\hline 178 & 986 & 280 & 760 \\
\hline
\end{tabular} \begin{tabular}{l|l|l|l|}
\hline 718 & 986 & 280 & 768 \\
\hline
\end{tabular} \begin{tabular}{|l|l|l|l|}
\hline 1719 & 924 & 270 & 994 \\
\hline
\end{tabular} \begin{tabular}{|l|l|l|l|}
1720 & 909 & 203 & 934 \\
\hline
\end{tabular} 1721874 2502065 \begin{tabular}{|l|l|l|l|l|l|l|l|l|}
\hline 1722 & $93^{8}$ & 208 & 2027 \\
\hline
\end{tabular}

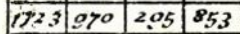
$\begin{array}{lll}172498 & 274 & 2150 \\ & \end{array}$ \begin{tabular}{|l|l|l|l|}
\hline 1725 & 932 & 286 & 921 \\
\hline 1928 & 968 & 288 & 959 \\
\hline
\end{tabular}

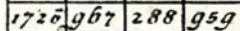
\begin{tabular}{|l|l|l|l|}
\hline $17 x 7966$ & 277 & 995 \\
\hline
\end{tabular} \begin{tabular}{|l|l|l|l|}
\hline 1728 & 984 & 336 & 2365 \\
\hline
\end{tabular} \begin{tabular}{l|l|l|l|}
\hline 1729 & 964 & 316 & 2323 \\
\hline
\end{tabular} $273006232 y$ 10g7 \begin{tabular}{|l|l|l|l|}
\hline $173^{1}$ & 1045 & 279 & 1040 \\
\hline
\end{tabular} \begin{tabular}{|l|l|l|l|}
\hline 2732 & 958 & 297 & 2348 \\
\hline
\end{tabular} \begin{tabular}{|l|l|l|l|}
\hline 2733 & 2007 & 309 & 1210 \\
\hline
\end{tabular} \begin{tabular}{|l|l|l|l|}
\hline 1734 & 2034 & 280 & 1172 \\
\hline 1755 & 990 & 848 & 1470 \\
\hline
\end{tabular} \begin{tabular}{|l|l|l|l|}
\hline 1735 & 999 & 243 & 1170 \\
\hline
\end{tabular} \begin{tabular}{|l|l|l|l|}
\hline 3736 & $107 x$ & 386 & 2279 \\
\hline
\end{tabular} \begin{tabular}{|l|l|l|l|}
\hline 1734 & 1073 & 317 & 1149 \\
\hline
\end{tabular} \begin{tabular}{|l|l|l|l|}
\hline 2735 & 1052 & 502 & 1141 \\
\hline
\end{tabular} \begin{tabular}{|l|l|l|l|l|}
\hline 739 & 1096 & 284 & 1390 \\
\hline
\end{tabular} \begin{tabular}{ll|l|l|l|}
1790 & 1031 & 280 & 1277 \\
\hline
\end{tabular}

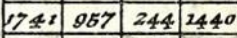
$\begin{array}{lllll}1742 & 948 & 283 & 1530\end{array}$ $\begin{array}{lllll}2743 & 947 & 297 & 1662\end{array}$

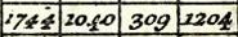
\begin{tabular}{|l|l|l|l}
\hline 745 & 1101 & 327 & 2272 \\
\hline
\end{tabular} \begin{tabular}{l|l|l|l|}
\hline 7740 & 1036 & 326 & 2274 \\
\hline
\end{tabular} \begin{tabular}{|l|l|l|l|l|}
\hline 1747 & 966 & 292 & 2227 \\
\hline
\end{tabular} \begin{tabular}{ll|l|l|l|l|l|l|l|}
17042 & 301 & 1233
\end{tabular} \begin{tabular}{|l|l|l|l|}
\hline 1740 & 1027 & 315 & 7175 \\
\hline
\end{tabular} \begin{tabular}{l|l|l|l|}
1750 & 1038 & 332 & 1270 \\
\hline
\end{tabular}

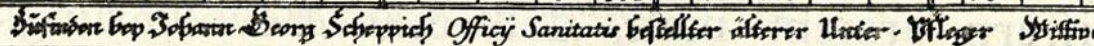

Abb. 19: Der Ausschnitt mit der Tabelle aus der Augsburger Bevölkerungstafel (1501-1750) in: FISCHER, Gesundheitswesen, Bd. I, S. 67, Abb. 6 
Laut dem kurzen Bericht in der zeitgenössischen Chronik war diese Krankheit mit starkem Husten und Brustschmerzen verbunden und befiel insbesondere viele reiche Leute in der $\operatorname{Stadt}^{430}$.

\section{Ausbruch der Pest im Jahr 1521 und Anweisungen Augsburger Stadtärzte}

Für die 1521/22 in Augsburg ausgebrochene Pestepidemie stellt sich dagegen die Überlieferung umfangreicher dar. Abgesehen von der allgemein immer dichter werdenden Quellenlage in der ersten Hälfte des 16. Jahrhunderts ergibt sich dieser Zustand vor allem aus den neuen Pestverordnungen des Rats, die von den Zeitgenossen sowohl in obrigkeitlichen Schriften wie auch in persönlichen Berichten erfasst wurden. Während dieser Epidemie wurden nämlich die institutionellen Grundlagen der städtischen Pestpolitik geschaffen, von denen die Pestbewältigung im frühneuzeitlichen Augsburg geprägt war.

Schon vor dem Ausbruch dieser Pestepidemie erließ der Rat im Herbst 1520 eine spezielle Anordnung zum Schutz der Stadtgemeinde vor Infektionen von außen ${ }^{431}$. Wegen der bestehenden Pestgefahr in der Umgebung durften nämlich fremde Bettler an den Feiertagen Allerheiligen und Allerseelen in Augsburg nicht eingelassen werden, um nach dem damaligen Brauch Almosen in der Stadt sammeln zu können. Diesmal mussten alle auswärtigen Bettler vor dem Wertachbrucker Tor außerhalb der Stadt verbleiben und dort auf die Austeilung der frommen Gaben von den Augsburgern warten. Zur Versorgung der versammelten Armen wurden vom Rat zehn Stapel Holz und zwei Karren mit Kohle geschickt und für jeden Bedürftigen ein Pfennig gespendet. In der Stadt selbst sammelten dagegen die städtischen Scharwächter vor allen Kirchen die Almosen von den Stadteinwohnern ein, die dann unter den fremden Bettlern vor der Stadt aufgeteilt wurden ${ }^{432}$.

Trotz dieser Vorsichtsmaßnahme begannen die ersten Pesterkrankungen in der Stadt im Juni 1521 und ab dem 25. Juli erreichte die Krankheit bereits ein epidemisches Ausma $\beta^{433}$. Kurz nach dem Ausbruch der Seuche wandten sich vier Doktoren Ambrosius und Ulrich

\footnotetext{
${ }^{430}$ StChr, Bd. 23, S. 451: In diesem Jahr starb hier füll reichs, gutz folck, als nie erhert was, mer denn armer, und was ain großer Husten unter dem Volk allenthalben und engnus um die Brust, als bei mans zeitten nit gehert was.

${ }^{431}$ SStBA, $2^{\circ}$ Cod. Aug. S 141, Fol. 118v-119v.

${ }^{432}$ Die Geldgaben vom Rat machten insgesamt 7 Gulden, 1 Kreuzer und 18 Pfennige aus. In der Stadt wurden dagegen von den Scharwächtern 44 Gulden, 43 Kreuzer und 4 Pfennige gesammelt, von denen zuerst den Alten je 1 Kreuzer und den Jungen 2 Pfennige, danach allen ohne Unterschied noch 1 Pfennig und der Rest den Kranken und den kinderreichen Bettlern ausgeteilt wurde.

${ }^{433}$ StChr, Bd. 23, S. 151; Bd. 25, S. 161; GASSER, Chronik, T. 3, S. 3.
} 
Jung, Sigmund Grimm und Hanns Trencklin, die damals als städtische Ärzte in Augsburg angestellt waren, mit einem Schreiben an den Rat, in dem sie ihre Vorschläge zur wirksameren Pestbekämpfung in der Stadt darlegten ${ }^{434}$. Nach Erkenntnis der Doktoren gab es zu diesem Zeitpunkt viele Anzeichen dafür, dass Gott in der nächsten Zeit auch Augsburg mit der Pest stärker heimsuchen könne. Es waren nämlich einige Häuser in der Stadt mit der Seuche befallen worden, in denen viele Leute schon daran gestorben waren. Aus diesem Grund wollten die eingeschworenen Doktoren dem Rat die notwendigen Maßnahmen für die Pestzeit schriftlich mitteilen und diesen nach Bedarf noch mit weiteren Ausführungen beraten. Weil die Pest wegen der Sünden der Menschen entstehen könne, solle man zunächst nach der Ansicht der Stadtärzte Gott mit Bittgebeten um seine Barmherzigkeit und Gnade anrufen. Mit derartigen Handlungen gegen die Pest war der Augsburger Rat aber sicher schon gut vertraut, wie die Mediziner bemerkten. Nach der Vollziehung der religiösen Praktiken sei es daher unbedingt nötig medizinische Maßnahmen zu ergreifen, mit denen man vor allem der weiteren Infektion innerhalb Augsburgs vorbeugen könne. Die Ärzte stellten in dieser Hinsicht fest, dass damals keine allgemeine Luftvergiftung in der Stadt herrsche und die Seuche sich nur aufgrund der direkten Übertragung von einem Menschen auf den anderen weiter verbreiten könne: kain gemaine fergifftung in lufft sey die mercklich gemain sey, sunder das hie von in fill orten $u \beta$ besondere ursach solche krankait umgeng und regirt, dar von den lederlich fergifft werden die so mit solichen personen handlend und wandlen auch bei wonend als sunderheist mugend beschedigen werden ${ }^{435}$. Demzufolge solle man die Häuser, in denen die Menschen von der Pest betroffen seien, sofort verschließen und die Gesunden am besten an andere Orte verlegen, damit sie von den Kranken nicht weiterhin vergiftet würden. Derartige infizierte Häuser sollen zudem dem Rat angezeigt werden, damit spezielle Verordnete in der Stadt die Aufsicht darüber haben, dass die Pestkranken bei Androhung einer Strafe ihre Häuser nicht verließen. Die Leute aus den umliegenden verpesteten Gebieten dürften dagegen generell nicht in die Stadt eingelassen werden.

Der Rat solle auch öffentlich bekannt machen, an welchen Orten man Hilfe bei Doktoren, Wundärzten, Badern und Barbieren in der Stadt fände. In der Stadt seien auch Pfleger zur Versorgung der Pestkranken zu verordnen, falls diese nicht in eine dazu bestimmte städtische Einrichtung aufgenommen werden können. Die Stadtärzte wiesen nämlich in ihrem Schreiben daraufhin, dass vom Rat ein spezielles Haus eingerichtet werden solle, in dem die Armen aus der Stadt untergebracht werden könnten. Dazu müsse man aber einen zweckmäßigen Ort

\footnotetext{
${ }^{434}$ StAA, CM IV, Nr. 7: Schreiben der vier Augsburger Stadtärzte (1521).

${ }^{435}$ Ebd.
} 
auswählen, damit die Stadtgemeinde durch die Existenz eines solchen Hauses keinesfalls beeinträchtigt würde. Bei der Planung eines städtischen Pesthospitals wollten die Stadtärzte daher die Ratsherren weiterhin beratend unterstützen: Es wirt auch von neten sein so dis krankait um werd gon und gemain werden das ain ersamer raut ain aigen huis da zu ferordneten da hin man solich arm und krancken thon mecht, das bedarff auch ain quemliche stat und ort da von gemaine statt nit beschediget wurde, wie dan durch doctores und bülat herwelt mag werd und die gemach reich kemlikait und notturfft fersehen ${ }^{436}$. Für die medizinische Versorgung in jenem Haus sollten einige Leib- und Wundärzte dorthin verordnet werden, die dann nur diese Kranken $\mathrm{zu}$ behandeln hätten und wegen der Infektionsgefahr nicht zu den gesunden Menschen gehen dürfen. Gleichzeitig solle auch das dortige Pflegepersonal unbedingt mit den notwendigen Arzneimitteln für seine eigene Prophylaxe versorgt werden.

Darüber hinaus solle der Rat auch spezielle Maßnahmen vornehmen, um die Menschen möglichst vor übermäßigem Schrecken zu schützen. Nach Ansicht der Stadtärzte können die Menschen beim Empfinden großer Furcht desto leichter von der Krankheit befallen werden. Aus diesem Grund dürften die Pestopfer nicht unter allzu großer Anteilnahme der städtischen Öffentlichkeit, d.h. in einem Prozessionszug quer durch die Stadt, begraben werden. Außerdem sei beständig darauf zu achten, dass die Luft in der Stadt weiterhin rein gehalten wurde. Gräber in der Stadt sollen während der Pestepidemie generell nicht ausgehoben bzw. geöffnet werden, sondern dazu sei ein bestimmter Ort zu verordnen, in dem man alle Verstorbenen gleichzeitig hineinlegen könne. Die Reinigung der Gassen und insbesondere der Orte, aus denen sich schlechte Gerüche erhoben, wie auch die Beseitigung der Misthaufen von den Schweinezuchten in der Stadt stammend, sei ebenfalls äußerst dringend zu raten. Mit derartigen Anweisungen legten die Augsburger Stadtärzte den Ratsherren einen Maßnahmenkatalog für die Pestzeit vor, der die wichtigsten Grundsätze der damaligen Pestlehre beinhaltete. Abschließend betonten die Mediziner in ihrem Schreiben noch einmal, dass sie ganz nach Belieben des Rates bereit wären, in dieser Hinsicht weitere Ratschläge zu erteilen.

\footnotetext{
${ }^{436}$ Ebd.
} 


\section{Die spezielle Sitzungsordnung des Rates und die Pestflucht der Augsburger} im Jahr 1521

Wie im Folgenden dargestellt wird, konnten sich die oben erläuterten Vorschläge der Mediziner rasch unter den Augsburger Stadtherren verbreiten und weitestgehend durchsetzen. Im Jahr 1521 wurden nämlich vom Rat umfassende Maßnahmen zur Pestbewältigung ergriffen, deren Grundlagen in erster Linie auf den von den Stadtärzten erteilten Anweisungen beruhten $^{437}$. Für die Dauer der Pestepidemie wurde zunächst die Tätigkeit des Augsburger Stadtregimentes auf eine besondere Art und Weise geregelt ${ }^{438}$. Am 3. September 1521 wurde eine spezielle Sitzungsordnung des Rates für die nächsten Monaten festgelegt. Danach solle jeweils nur ein Drittel der gewöhnlichen Zusammensetzung des Rates Sitzungen abhalten, damit die anderen Ratsherren in dieser Zeit vorsorglich die Stadt verlassen könnten. Dies wolle man bis zum Ende der Epidemie - bei monatlichem Wechsel - so durchführen ${ }^{439}$. Dasselbe galt auch für die Verwalter der drei wichtigsten Ratsämter in Augsburg: Baumeister, Einnehmer und Ungeldherren, die vornehmlich für die finanziellen Angelegenheiten der Stadt zuständig waren ${ }^{440}$. Von diesen Ämtern solle jeweils nur ein Amtsträger vor Ort bleiben, um die wichtigsten laufenden Amtsgeschäfte zu verwalten. Am 10. Oktober 1521 stellten die Steuerherren auch die Einschreibung der steuerpflichtigen Personen ein. Sie konnten ihre Tätigkeit jedoch bereits am 19. Januar 1522 wiederaufnehmen, da die Seuche zu diesem Zeitpunkt merklich abgeklungen war ${ }^{441}$.

Die zeitweise von ihren Amtspflichten befreiten Stadtherren konnten sich also für einen Zeitraum von höchstens zwei Monaten aus der Stadt in die pestfreie Nachbarschaft begeben. Dass sie diese Möglichkeit in Anspruch nahmen, kann man anhand der zeitgenössischen

\footnotetext{
${ }^{437}$ Zum allgemeinen Hintergrund der Umwandlungen in der städtischen Pestpolitik in Augsburg, siehe Kapitel VIII, S. 173f.

${ }^{438}$ Unter dem Stadtregiment in Augsburg ist vor allem der Kleine Rat als die politisch wichtigste Institution in der Stadt zu verstehen, die seit 1368 mehrheitlich mit Zunftvertretern besetzt war. Nach der Regimentsreform Kaiser Karls V. im Jahr 1548 stellten die Patrizier wieder einen überwiegenden Teil der Ratsherren, unter denen die Katholiken ein Übergewicht im bikonfessionellen Augsburg besaßen. Aus dem Kleinen Rat wurden auch die Verwalter der städtischen Ratsämter erwählt, deren wichtigste Vertreter auch zu einem Sonderausschuss des Kleinen Rates als die „Dreizehner“ und nach 1548 als der „Geheime Rat“ zusammenkamen. Der Große Rat erfüllte dagegen vor allem die gesellschaftliche Integrationsfunktion und nahm nicht an der alltäglichen Stadtverwaltung teil. Zur Verfassungsgeschichte Augsburgs: RogGE, Für den Gemeinen Nutzen; RoECK, Krieg und Frieden; BÁTORI, Augsburg; WARMBRUnN, Konfessionen.

${ }^{439}$ StAA, Ratsprotokolle, 1521, Fol. 11-12. Die ersten Beschränkungen der Flucht der Ratsherren vor der Pest sollten laut den chronikalischen Berichten schon 1438 beschlossen werden, siehe S. 35 .

${ }^{440}$ Das Baumeisteramt fungierte in seiner Bedeutung und nach der Zahl der Angestellten als das wichtigste Ratsamt in Augsburg, dessen Aufgaben die direkte Verwaltung des gesamten städtischen Ausgabewesens betrafen. Die Einnehmer, Ungeld- und Steuerherren, die neben den Baumeistern zu den bedeutendsten Stadtbeamten in Augsburg gehörten, waren dagegen für die Einnahmen der Stadtkasse zuständig. Vgl. dazu in: RoECK, Krieg und Frieden, Bd. 1, S. 264-267; BÁTORI, Augsburg, S. 63-64.

${ }^{441}$ StAA, Ratsprotokolle, 1521, Fol. 12; 1522, Fol. 15.
} 
chronikalischen Berichte annehmen. Laut den zeitgenössischen Schilderungen von Clemens Sender (1475-1536), einem Mönch des St. Ulrich und Afra Klosters, flohen 1521 viele reichen Augsburger vor der Pest nach Ulm, Donauwörth und Lauingen ${ }^{442}$. Ihre Anzahl wurde von Sender auf 1400 Personen geschätzt. Die Details über die Pestflucht der Augsburger im Jahre 1521 stellte dagegen Wilhelm Rem (1462-1529), ein vermögender Kaufmann zusammen, der zu einer der bedeutendsten Familien in Augsburg gehörte ${ }^{443}$. Laut seinen Schilderungen begannen die Leute am 24. August 1521 wegen der sich in der Stadt verbreitenden Epidemie in zahlreiche Orte in der Umgebung von Augsburg zu fliehen. Die bevorzugten Zielorte waren dabei vor allem die drei oben genannten Nachbarstädte, in denen man jedoch hohe Hausmieten für schlechte Wohnbedingungen zahlen musste. In Lauingen und Ulm waren dementsprechend 50 bzw. 96 Häuser von den Leuten aus Augsburg belegt, wobei zu einem späteren Zeitpunkt auch die Menschen aus Donauwörth, wegen des dortigen Ausbruchs der Pest, gleichfalls in die Stadt Ulm zogen. Die Augsburger Handwerker, die sich keine hohen Unterhaltskosten in den Nachbarstädten leisten konnten, zogen sich dagegen auf die Strassen in den umliegenden Dörfern zurück. Die beste Zuflucht während der damaligen Epidemie bot den Augsburger Bürgern vornehmlich die Stadt Ulm an, deren Rat allen hiesigen Zünften einen besonderen Empfang der Menschen aus Augsburg befahl ${ }^{444}$. Zur Verdeutlichung der dort erfahrenen Gastfreundschaft erwähnte Wilhelm Rem eine feierliche Mahlzeit auf der Trinkstube am 16. Oktober 1521 in Ulm, zu der ca. 50 Augsburger Kaufleute und Bürger neben einigen Pestflüchtlingen aus anderen Städten eingeladen worden waren. Anlässlich dieses festlichen Empfangs bezahlten die Augsburger vier Gulden für die Speisen und zwei Gulden für die Musikanten. Angesichts derartiger Festlichkeiten in Ulm scheint es möglich zu sein, dass unter den eingeladenen Augsburgern auch einige Ratsherren dort anwesend waren. Die mögliche Flucht eines größeren Teils von Machtträgern aus Augsburg beeinträchtigte aber keinesfalls die Entscheidungsfähigkeit des Stadtregimentes in der Pestzeit. Trotz der damals vorgenommenen Arbeitsteilung der Ratsherren konnten die entscheidenden Maßnahmen zur städtischen Pestbewältigung in Augsburg weiterhin ergriffen werden.

\footnotetext{
${ }^{442}$ StChr, Bd. 23, S. 152.

${ }^{443}$ StChr, Bd. 25, S. 161-162, 165-166.

${ }^{444}$ Zuflucht vor der Pest in Ulm fand auch Lucas Rem (1481-1541) mit seiner Familie, der ein ferner Verwandter von Wilhelm Rem war. Nach Augsburg kam die Familie Rem erst am 21. März 1522 zurück. Während der nächsten Epidemie im Jahre 1535 flohen sie mit ihrem Hausgesinde wieder nach Ulm, wo sie ein geräumiges Haus von Sebolt Stoffel Giengers mieteten. Zuerst schickte Lucas Rem am 12. August seine Mutter mit seinen vier Kindern und einem Teil der Dienstmägde dorthin und dann verließ er selbst am 17. August die Stadt mit seiner Frau und dem Rest der Dienstleute. Nach Augsburg kamen sie am 19. Februar 1536 zurück, in: REM, Tagebuch, S. 23-24, 27-28, 65.
} 


\section{Gründung des Pesthospitals („Brechhäuser“) mit einem Friedhof im Jahr 1521}

Der gewichtigste Ratsbeschluss von 1521 war die Gründung eines großen Pesthospitals außerhalb der Stadt. Die neue Hospitalanlage bestand aus zwei Häusern, die entsprechend für die Kranken und für die Rekonvaleszenten vorgesehen waren. In der Überlieferung aus der ersten Hälfte des 16. Jahrhunderts wurden sie allgemein „Brechhäuser“ genannt ${ }^{445}$. Diese zeitgenössische Bezeichnung wurde dabei von dem Begriff brechen abgeleitet, der in der Augsburger Schriftsprache als Synonym für die Pest verwandt wurde. In der zweiten Hälfte des 16. Jahrhunderts kam zusätzlich der Begriff „Lazarett“ in Gebrauch, der nach dem Vorbild des berühmten Venezianischen Pesthospitals „Lazaretto vecchio“ auch von den Augsburgern für ihre städtische Einrichtung gebraucht wurde ${ }^{446}$. Diese neue Hospitalanlage befand sich außerhalb der Stadt im Lugißland vor dem Stephingertor, wodurch man, nach der Beratung durch die Stadtärzte, eine völlige Isolierung der dort versorgten Pestkranken von der Stadtgemeinde umsetzen konnte. Ihre abgelegene Lage ist auf dem Stadtplan in der Chronik von Paul von Stetteten zu erkennen (Abb. 20). Im rechten Teil des Bildes sind die beiden Häuser als Lazarett neben der erst zwischen 1611-1613 erbauten St. Sebastian-Kirche nicht weit von dem Ort gekennzeichnet, wo die Flüsse Lech und Wertach ineinanderfließen. Diese beiden Gebäude hatten ursprünglich je ein Erdgeschoß und waren 125 Schuhe lang und 30 breit. Laut den Angaben in der Pestschrift der Augsburger Stadtärzte konnte man dort im Jahre 1535 fast 150 Personen behandeln ${ }^{447}$.

\footnotetext{
${ }^{445}$ StChr, Bd. 23, S. 151; Bd. 25, S. 163; GASSER, Chronik, T. 3, S. 3; STETTEN, Geschichte, VIII. 13. 288; SStBA, $2^{\circ}$ Cod. Aug. 214, T. IV, Supl. T. III., 1521; StAA, Ratsprotokolle, 1521, Fol. 14.

${ }^{446}$ Das Lazaretto Vecchio wurde 1423 in Venedig auf einer kleinen Insel in der Lagune als das erste kontinuierlich unterhaltende Pesthospital der Welt errichtet. Der Begriff "Lazarett" entstand nach der Ansicht von Klaus Bergdolt aus der Vermischung des Namens des alten Klosters Santa Maria di Nazareth, auf dessen Insel nun das Pesthospital gegründet worden war, mit demjenigen der dem Heiligen Lazarus geweihten benachbarten Aussätzigeninsel. Nach dem Venezianischen Vorbild der institutionellen Fürsorge für die Pestkranken wurden bis zum Ende des 15. Jahrhunderts die weiteren Pesthospitäler in vielen italienischen Städten, insbesondere auf dem Venezianischen Territorialgebiet eingerichtet. Dazu in: BERGDOLT, Die Pest, S. 76; PALMER, The Control, S. 183-189.

447 OCCO/SAYLER/TIEFFENBACH/TALHAUSER, Was die Pestilentz an jr selbs sey [Augsburg: Philipp Ulhart 1535], S. E IIIv.
} 


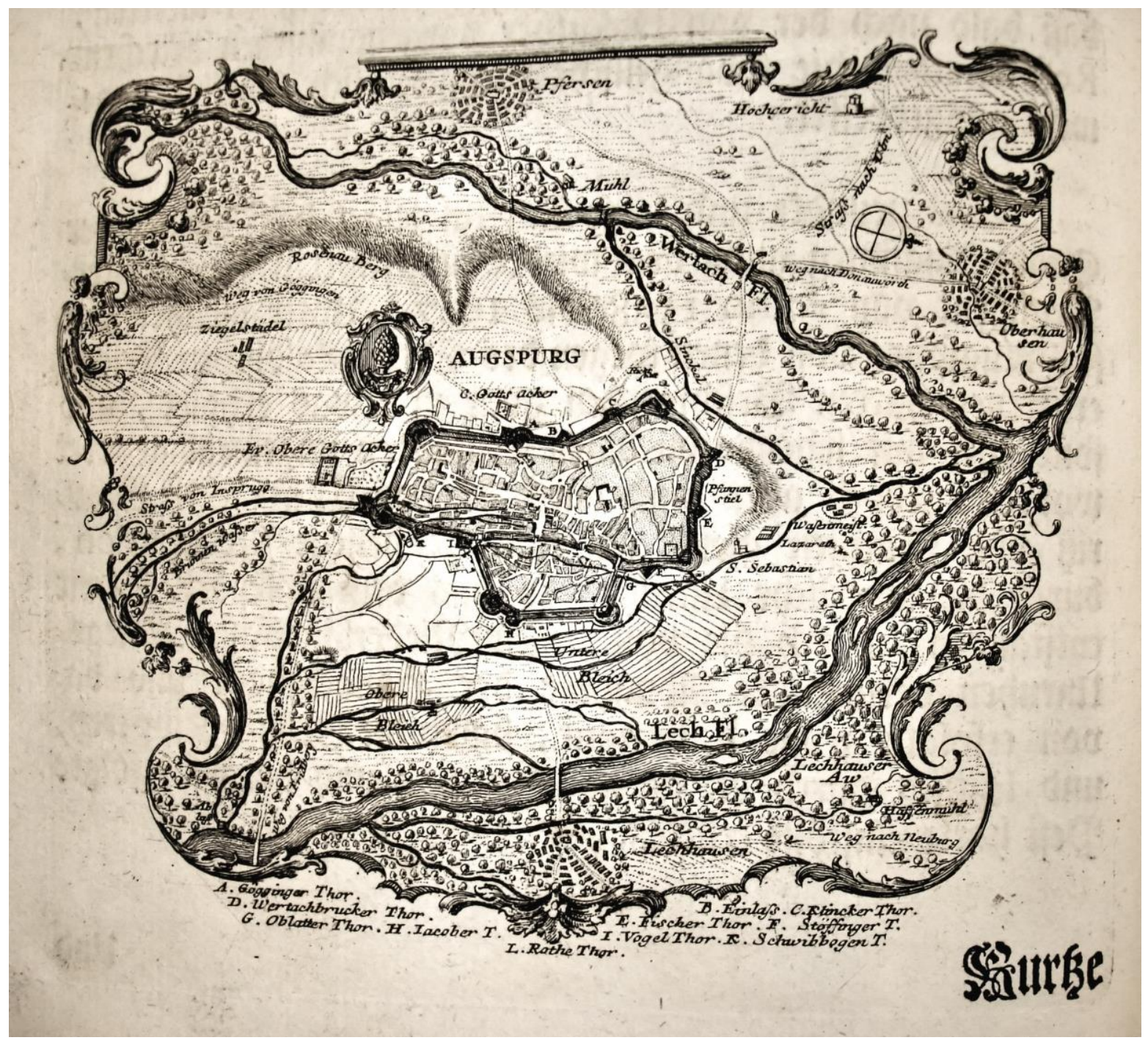

Abb. 20: Augsburger Stadtplan in der ersten Hälfte des 18. Jahrhunderts, in: Stetten, Geschichte der Heil. Röm. Reichs Freyen Stadt Augsburg, Frankfurt/Leipzig 1743

In den ersten Jahren des 17. Jahrhunderts wurde das Pesthospital unter der Leitung des Baumeisters Elias Holl großflächig umgebaut ${ }^{448}$. Die ursprüngliche Konstruktion aus Holzund Lehmwerk wurde durch Steinmauerwerk ersetzt und die beiden Häuser auf 146 Schuhe Länge und 46 Breite vergrößert. Dazu erbaute man auf jedem Haus ein weiteres Stockwerk, das jeweils in drei Stuben aufgeteilt wurde. In zwei von diesen Stuben wurden damals die nach der katholischen und evangelischen Konfession eingeteilten Rekonvaleszenten in den Brechhäusern gepflegt. Im Jahr 1611 begann dagegen der Bau der Hospitalkapelle und daneben eines kleines Hauses, in dem während der Pestepidemien die anscheinend als

\footnotetext{
${ }^{448}$ MEYER (Hrsg.), Die Hauschronik der Familie Holl, S. 54-55. Laut den Aufzeichnungen von Elias Holl wurde der Umbau des Pesthospitals etlich wenig jahr vor dem Jahr 1611 durchgeführt.
} 
Seelsorger der kranken Katholiken tätigen Kapuzinermönche wohnten ${ }^{449}$. Diese Hospitalanlage wurde nach dem Umbau anschaulich im Kupferstich von Raphael Custos auf dem Flugblatt von 1629 dargestellt ${ }^{450}$. Im Zentrum des Bildes (Abb. 21) erstreckt sich die Heerd-Gasse (heutige Sebastianstraße), die von der Vorstadt aus direkt zum Stephingertor führt. Dieser Feldweg ist rechts vom hohen Zaun begrenzt, der mehrere Gärten und Felder umschließt. Links befindet sich dagegen das von Zäunen und Mauern geschützte Gelände des Pesthospitals. Lediglich die Fassade der 1613 geweihten St. Sebastian-Kirche unterbricht die umgezaunte Front des gesamten Hospitalgeländes. Dahinter steht ein kleines Haus für die Kapuziner. Über Zaun und Mauer hinweg sind links vorne zwei große Gebäude mit Satteldach zu sehen, in denen die Pestkranken während der Epidemien versorgt wurden ${ }^{451}$.

Die 1521 erbauten Brechhäuser waren gleichermaßen wie frühere derartige Einrichtungen vornehmlich zur Versorgung der Armen in der Stadt eingerichtet worden. Dabei konnten in ihnen offensichtlich nicht alle kranken Mägde und Knechte während der Pestzeit aufgenommen werden. In den Verordnungen des Rates aus dem Jahr 1535 versuchte man auch dieses Problem zu regeln ${ }^{452}$. Wenn jemand seine erkrankten Dienstleute nicht im Haus behalten wolle, dürfe man diese wie von alter her gewesen wegbringen. Man solle die Kranken jedoch mit Vorsicht und Vernunft an einen dazu geeigneten anderen Ort verlegen, an dem möglichst wenig Gefahr für die anderen Menschen bestehe.

\footnotetext{
${ }^{449}$ In dem bei der Kapelle erbauten Haus befanden sich 4 Stuben, 4 Kammer, 2 Küchen, eine Sakristei und ein gewölbter Keller.

${ }_{450}$ Christliche Erinnerung [Augsburg: Johannes Schultes 1629], HARMS, Flugblätter, Bd. 1, Nr. 236.

${ }^{451}$ In den oberen Bildecken schweben unter Wolken zwei Engel, deren Beischriften (links BELLUM, FAMES, PESTIS; rechts MISERICORDIA; GRATIA; BONITAS) und Attribute (Flammenschwert bzw. Buch und Palmwedel) sie als Verkörperungen des strafenden und lohnenden Gottes ausweisen. Diese Eigenschaften des göttlichen Richters werden noch einmal mit den beiden Engeln zugeordneten Bibelzitaten hervorgehoben (Jeremias 14, V, 12: So spricht der Herr von diesem Volck: Ich Will Sie mit dem Schwert, Hunger und Pestilenz aufreiben; Gottes Güte ist es das Ihr nicht gar aus seit Thren 3.V, 22; Dann Barmhertzig und Gnadig ist der Herr, Gedultig und von grosser Güte, Psalm 103, V, 8). Auf der linken Seite des Himmels befindet sich dagegen ein Komet, mit dessen Erscheinen die Strafe Gottes vorangekündigt wird. Das Wappen Augsburgs in der Himmelsmitte verweist auf den Ort des Geschehens.

${ }^{452}$ StAA, Schätze, Nr. 16, p. 8R-11: Pestverordnungen des Rates pro Memoria (1535); StAA, CM IV, Nr. 13: Pestverordnungen des Rates (1535).
} 


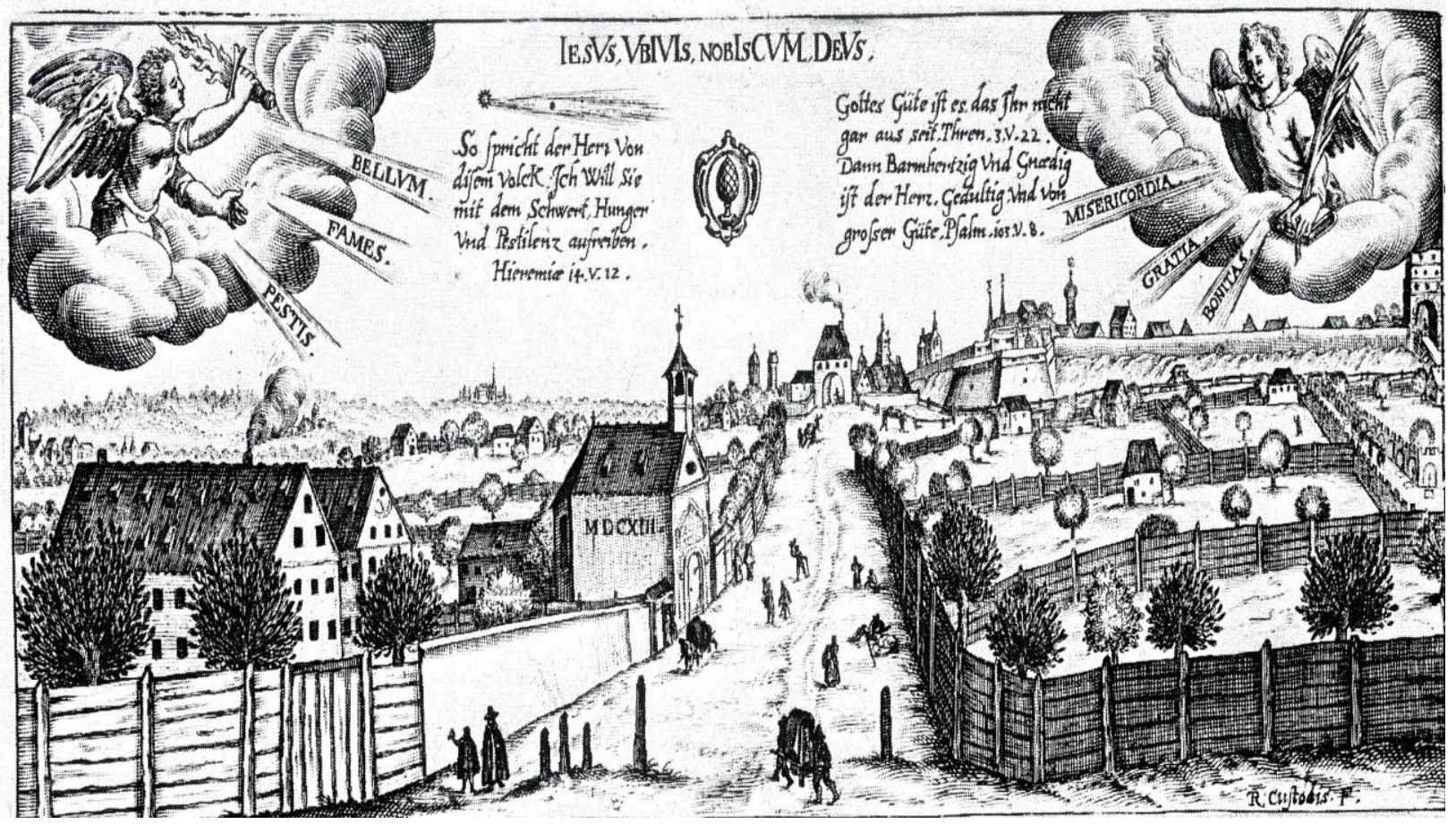

Abb. 21: Die Pesthospitalanlage in Augsburg (Kupferstich von Raphael Custos) aus dem Einblattdruck Christliche Erinnerung [Augsburg: Johannes Schultes 1629], in HARMS, Flugblätter, Bd. 1, Nr. 236

Die Errichtung der neuen Hospitalanlage war mit einer hohen finanziellen Belastung für die Stadt verbunden. Um die enormen Baukosten decken zu können, wurden 1521 zunächst aus dem Stiftungsvermögen von zwei anderen städtischen Hospitälern beträchtliche Summen verwendet. Aus den Pflegschaften von St. Sebastian-Siechenhaus und St. Jakob-Hospital wurden auf Anordnung des Rates jeweils 900 bzw. 600 Gulden entnommen ${ }^{453}$. Darüber hinaus wurden damals die Unterhaltskosten der neuen Pesthäuser auch von den Almosengaben der Stadtbewohner getragen. In den Pestverordnungen des Rates aus dem Jahr 1521 findet sich dazu eine Instruktion für alle städtischen Priester, dass sie während der Predigten die Leute besonders dazu ermuntern sollten, zum Unterhalt der neuen städtischen Einrichtung fromme Gaben zu stiften, damit die dort untergebrachten Kranken besser versorgt würden ${ }^{454}$.

Die Stiftungsbereitschaft der Augsburger war sicher noch steigerungsfähig, als am 6. November 1521 eine Bulle bezüglich der neuen Hospitalanlage von Papst Leo X. erlassen

\footnotetext{
${ }^{453}$ StAA, Ratsprotokolle, 1521, Fol. 13-14. Zu den Sankt Sebastians und Sankt Jakobs Pflegen in: KIEßLING, Bürgerliche Gesellschaft, S. 169-174; HERBERGER, Die St. Jakobs-Pfründe; Zur allgemeinen Entwicklung des Augsburger Hospitalwesens, siehe S. 187-191.

${ }^{454}$ StAA, CM IV, Nr. 13: Pestverordnungen des Rates (aus dem Inhalt datierbar für 1521).
} 
wurde $^{455}$. In dem schon erwähnten Bittschreiben des Rates ersuchte man die päpstliche Zustimmung für die neuen Regelungen des Begräbniswesens und der Seelsorge in Augsburg, die angesichts der neuen städtischen Maßnahmen in der Pestzeit erforderlich waren ${ }^{456}$. Die Ratsherren berichteten darin, dass es für die im Vergleich zu anderen deutschen Städten größere Bevölkerung in Augsburg an ausreichenden Begräbnisplätzen fehle. Die Verstorbenen aus den außerhalb der Stadtmauern gelegenen Brechhäusern sollten daher nicht auf den innerstädtischen Kirchhöfen begraben werden, damit die Stadt wegen des nächtlichen Toreschließens sowie aufgrund anderer wichtiger Ursachen nicht beeinträchtigt werde. Aus diesen Gründen beschloss der Rat, auf einigen Gärten in der nächsten Umgebung des neuen Pesthospitals noch einen Friedhof und eine Kapelle einzurichten. Dadurch konnten die Kranken direkt vor Ort mit der Seelenfürsorge versehen werden und die im Hospital Verstorbenen wie auch die Leute, die ihr Grab aus freiem Willen auf diesem Friedhof haben wollten, begraben werden.

Außerdem wies man darauf hin, dass schon rein mengenmäßig nicht alle Kranken aus der Stadt in die außerstädtischen Brechhäuser verordnet werden konnten, sondern abgesondert in ihren eigenen Häusern versorgt werden müssten. Daher wollten die Ratsherren künftig, wenn es notwendig sei, in der Vorstadt oder außerhalb der Stadt noch weitere Friedhöfe oder Kapellen gründen, damit an diesen Orten auch die anderen Verstorbenen aus der Stadt bestattet werden könnten. Nach der Beurteilung des Rates sollten nicht alle Leichen auf dem neuen Friedhof bei den Brechhäusern begraben werden, weil sich dort aus der Vielzahl der begrabenen Körper die Infektion weiter vermehren oder wieder erheben könne.

Bei der zukünftigen Durchsetzung solcher Verordnungen wollten die Ratsherren offensichtlich nicht länger von der Einwilligung der hiesigen Kirchherren abhängig sein, die wegen der möglichen Verminderung ihrer Einkünfte solche Maßnahmen in der Pestzeit zu verhindern suchten. Der Rat beanspruchte jetzt, die gesamte städtische Pestpolitik allein bestimmen zu können und nicht mehr, wie es noch im Jahre 1494 geschehen war, mit dem Bischof, dem Domkapitel und allen Pfarr- und Kirchherren in Augsburg vereinbaren zu müssen. Nach dem Abschluss des Vertrages über die Gründung des St. Stephans-Gottesackers gab es danach einige Streitigkeiten zwischen den städtischen und kirchlichen Parteien um die Verteilung der Gefälle und um die Präsentationsrechte für den anzustellenden Geistlichen, die

\footnotetext{
${ }^{455}$ SStBA, $2^{\circ}$ Cod. Aug. 214, T. IV, Supl. T. III: Pabst Leo X. Bulle, die 2 Brechhäuser ausser der Stadt, Capellen, Kirchhöfe, und Priesterschaft dabei betreffend (6. November 1521).

${ }^{456}$ SStBA, $2^{\circ}$ Cod. Aug. 215, T. IV, Supl. T. III., 1521: Des Raths zu Augspurg Bittschreiben um eine Bulle über die 2. Brechhäuser, an Pabst Leo X.
} 
endgültig erst mit einem neuen Vertrag am 13. Mai 1508 beendet wurden ${ }^{457}$. Angesichts des vergangenen Streites ersuchte der Rat in seinem Bittschreiben den Papst um eine generelle Approbation, dass die künftig zu gründenden Friedhöfe, falls der Bischof oder der Suffragan damit nicht einverstanden wären, auch von einem Priester oder Mönch eingeweiht werden könnten. Der Rat wollte auch das Patronatsrecht für sich behalten, wenn man jetzt oder in der Zukunft eine feste oder befristete Pfründe für die Priester in den neuen Kapellen auf den Friedhöfen stiftete. Demgemäß sollten die dazu verordneten Kapläne seither nur dem Papst untergeordnet werden. Die Bittgesuche des Rates wurden von Papst Leo X. in seiner Bulle beantwortet und erfüllt. Die städtische Obrigkeit in Augsburg konnte daher die wichtigsten Angelegenheiten der Seelsorge und des Begräbniswesens in der Pestzeit auch ohne Mitspracherecht der hiesigen Kirche regeln.

\section{Die Ordnung für die Brechhäuser von 1521}

Aus dem Jahr 1521 ist die erste Ordnung für die Brechhäuser überliefert, auf deren Grundlage ihre allgemeine Verwaltungsstruktur und Organisation $\mathrm{zu}$ erschließen ist ${ }^{458}$. Die Oberste Instanz für diese städtische Einrichtung war der Rat, der letztlich über alle ihre Angelegenheiten entscheiden konnte. Zur direkten Administration dieser neuen Hospitalanlage sollten vom Rat zwei Pfleger ernannt werden, die vor allem für die finanziellen und organisatorischen Aufgaben zuständig waren. Diese Verwaltungspflichten wurden anscheinend schon von Anfang an den Pflegern des städtischen Blatterhauses auferlegt, die sich aus dem Kreis der Zünfte rekrutierten ${ }^{459}$. Einen Hinweis darauf geben die Ratsprotokolle aus dem Jahr 1521, laut deren die Besoldung des im Brechhaus angestellten Priesters von den Pflegern des Blatterhauses ausgezahlt wurde ${ }^{460}$. Aus den Verordnungen des Rates aus dem Jahr 1535 geht dagegen eindeutig hervor, dass diese Stadtbeamten mit der Versorgung der Brechhäuser und mit der Verwaltung des dortigen Personals beauftragt waren $^{461}$. Ihre darüber abgefassten Berichte mussten sie direkt dem Rat oder den Bürgermeistern vorlegen. Die Pfleger des Blatterhauses fungierten also seither auch als

\footnotetext{
${ }^{457}$ KIEßLING, Bürgerliche Gesellschaft, S. 128-131.

${ }^{458}$ StAA, CM IV, Nr. 13: Pestverordnungen des Rates (aus dem Inhalt datierbar für 1521).

${ }^{459}$ Die Pfleger, die ursprünglich nur zur Verwaltung des Blatterhauses vom Rat eingesetzt wurden, sind schon seit dem Jahr 1495 bezeugt, dazu in: STEIN, Franzosenkrankheit, S. 122.

${ }^{460}$ StAA, Ratsprotokolle, 1522, Fol. 22-23.

${ }^{461}$ StAA, CM IV, Nr. 13: Pestverordnungen des Rates (1535).
} 
Mittlerinstanz zwischen den Brechhäusern und dem Rat ${ }^{462}$. Die administrative Verknüpfung dieser Institutionen könnte dabei aus der früheren Organisation der städtischen Pestfürsorge hervorgegangen sein, als die Franzosen- und Pestkranken während der Epidemien vor 1521 noch gemeinsam in zwei Häusern in der Jakobervorstadt versorgt wurden.

Nach der damaligen Pesthausordnung lag die alltägliche Verwaltung der Häuser vor Ort in den Händen des Unterpflegers und der Unterpflegerin, die aber in späteren Quellen allgemein als Brechvater und Brechmutter bezeichnet wurden ${ }^{463}$. Zur Durchführung solcher Aufgaben wollte der Rat nur Personen ernennen, die lese-, schreib- und rechenfähig waren, um das notwendige Rechnungswesen in den Brechhäusern führen zu können. Der Brechvater und die Brechmutter waren im Turnus von vierzehn Tagen verpflichtet, alle Einnahmen und Ausgaben für die Küche und andere Bedürfnisse der Häuser schriftlich zusammenzustellen und die Rechnungen den Pflegern einzureichen. Diese sollten anschließend die erhaltenen Abrechnungen überprüfen und wenn es von ihnen gefordert wurde, die entsprechenden Akten auch dem Rat vorlegen. Nach der Neuanstellung des Brechvaters wurde jeweils von zwei ehrsamen Personen ein Inventar allen vorhandenen Hausrates in den Brechhäusern aufgestellt, deren Abschriften von den Pflegern bzw. auch vom Rat aufbewahrt wurden. Das Inventar sollte dann alle Vierteljahre oder nach Bedarf von den Pflegern nachgeprüft werden.

Für die Versorgung der Brechhäuser waren Dienstleute wie Köche, Mägde und Knechte notwendig, die außer ihrem monetären Lohn auch freie Kost für sich selbst erhalten sollten. Man berief auch einen Priester zur Seelsorge der Kranken, der sie zu Beichte ermahnen und mit den Heiligen Sakramenten versehen solle ${ }^{464}$. Zur medizinischen Behandlung der Kranken wurde damals ein Barbier angestellt, der nur in den Brechhäusern tätig sein solle. Dagegen standen die Stadtärzte in keinem festen Dienstverhältnis, sondern gingen nach Bedarf zu den dortigen Pestkranken. Sie verordneten nämlich neben dem Barbier alle Heilmittel in den Brechhäusern, die aus bestimmten Apotheken in der Stadt für erschwingliche Preise zugestellt werden sollten.

In das Pesthospital durften nur diejenigen Kranken aus der Stadt aufgenommen werden, bei denen die Mediziner eindeutig die Pest diagnostiziert hatten. Davon waren allerdings Kinder

\footnotetext{
${ }^{462}$ Nach der sog. Carolingischen Regimentsreform im Jahr 1548 wurden dagegen zur Verwaltung des Blatterund Brechhauses zwei patrizischen Oberpfleger aus dem Kleinen Rat ernannt, denen seither diese zünftischen Pfleger als „Unterpfleger“ direkt unterstanden, vgl. dazu in: STEIN, Franzosenkrankheit, S. 122-123.

${ }^{463}$ StAA, CM IV, Nr. 13: Pestverordnungen des Rates (aus dem Inhalt datierbar für 1521).

${ }^{464}$ Das Bittgesuch des Priesters um eine feste Pfründe wurde am 29. September 1522 vom Rat abgelehnt. Die Pfleger des Blatterhauses sollten ihm nur einen halben Gulden bis zu dem Zeitpunkt auszahlen, an dem er endgültig vom Rat aus seinem Dienst entlassen werden würde. Die Barbiere, die zur Durchführung des Aderlasses in der Stadt angestellt waren, wurden dagegen bereits zu diesem Zeitpunkt vollständig entlassen, in: StAA, Ratsprotokolle, 1522, Fol. 22-23.
} 
unter drei Jahren ausgenommen, die trotz ihrer Erkrankung dort nicht behandelt werden sollten. Zur besseren Kontrolle der Aufnahme sollte der Barbier alle ankommenden Kranken fleißig beschauen, damit man die mit anderen Krankheiten befallenen Personen sofort aus den Brechhäusern wegschicken konnte. Wenn ein mutmaßlicher Kranker noch keine äußerlichen Pestzeichen hätte, sollte dieser zunächst von einem der Stadtärzte untersucht werden, weil diese in der Lage waren, auch aufgrund der anderen Symptome die Erkrankung zu erkennen. Der Rat betonte, dass die Pestkranken in keine anderen Hospitäler der Stadt aufgenommen werden dürfen, damit sich die Seuche dadurch nicht weiter verbreiten könne. Die Brechhäuser sollten dagegen von allen übrigen Stadteinwohnern völlig isoliert werden. Deswegen durfte niemand ohne die Erlaubnis des Brechvaters bei der Strafandrohung eines halben Guldens dort eingelassen werden. Zum Transport der Kranken und der Verstorbenen benutzte man speziell angemalte Wagen, damit diese von den Leuten leicht erkannt und dadurch immer gemieden werden konnten. Dazu ordnete der Rat eine genaue Kennzeichnung der Wagen mit zwei Farben an: schwarze Farbe für die Toten und rote Farbe für die Kranken. Die Lebensmittel und andere notwendige Sachen würden dagegen von einer vom Rat verordneten Person geliefert, die jeden Tag alles Nötige auf einer Karre in die Brechhäuser führen solle.

Nach der Aufnahme ins Pesthospital wurden die Kranken ihrer eigenen Kleidung entledigt und dann in ein vom Barbier und dem Brechvater gemeinsam verordnetes Bett gelegt. Ihre Kleidung und sonstige Sachen seien dabei in den Gemächern der Kranken aufzubewahren, damit man sie ihren Inhabern im Fall einer Genesung wieder zurückgeben konnte. Alle Waffen, die von Kranken mitgebracht wurden, mussten dagegen während ihres Aufenthaltes zur Sicherung der Hausordnung unter die Aufsicht des Brechvaters genommen werden. Nach dem Tod eines Kranken durfte seine Kleidung nicht mehr gebraucht werden, sondern musste ausnahmslos verbrannt werden. Auch wenn von den Insassen der Pesthäuser einiges Essen und Getränke übrig waren, durfte man es keinesfalls an andere Personen außerhalb des Hospitalgeländes weitergeben, sondern hatte alles in den Fluss zu schütten. Damit wollte man vermeiden, dass die Seuche mittels der von den Kranken gebrauchten Kleidung bzw. Lebensmittel nicht auf andere gesunde Menschen übertragen werden könnte.

Die Kranken und Rekonvaleszenten wurden jeweils in einem der zwei Brechhäusern getrennt voneinander versorgt, damit die genesenden Personen nicht mehr von den Kranken vergiftet werden konnten. Außerdem waren die Insassen nach Geschlechtern getrennt untergebracht, indem man die Frauen auf die Betten auf der Erde und die Männer auf die oberen Bettgestelle verwies. Die Rekonvaleszenten konnten dabei vom Brechvater zu keiner Arbeit auf dem Hospitalgelände herangezogen werden. In diesen Regelungen wurde eine besondere 
Vorgehensweise vermerkt, wenn die Insassen synnles wurden. Wenn jemand mehrmals in einen solchen Zustand geriet und dadurch die Ordnung in den Häusern gefährdete, solle er oder sie auf einer speziellen Bettstatt mit Bändern oder Ketten gefesselt werden.

Darüber hinaus durften die Kranken sowie das Hospitalpersonal in der Pestzeit wegen Angelegenheiten, die Hab und Gut betrafen, nicht vor das Stadtgericht geladen werden. Dagegen wurde vom Brechvater ein spezielles Buch geführt, in das man alle Geschäfte und Vermächtnisse der Insassen in Anwesenheit von zwei Zeugen mit Gesetzeskraft eintragen konnte. Davon durfte aber keinem Bediensteten ein Vorteil zukommen. Nach der Anordnung des Rates sollte diese Hausordnung auch in einem Buch niedergeschrieben und immer in den Brechhäusern aufbewahrt werden, damit sich die dortigen Angestellten strikt nach ihren Bestimmungen richteten.

Die Insassen in den Brechhäusern sollen keinesfalls nur von den Gesunden isoliert, sondern vor allem medizinisch versorgt werden, wodurch man zahlreiche Kranke zu heilen hoffte. Diese Überzeugung ist schon im Gründungsakt des neuen Pesthospitals erkennbar, als zur Versorgung der Kranken und der Rekonvaleszenten speziell zwei getrennte Häuser eingerichtet wurden. In den Bestimmungen der ersten Hausordnung tritt diese zeitgenössische Auffassung noch deutlicher zutage. Neben der Aufbewahrung der Kleidung von Insassen für den Fall ihrer Genesung wurde darin auch verordnet, dass die genesenen Personen nach ihrer Entlassung in ihre eigenen Häuser noch einige Zeit bis zur völligen Gesundung nicht unter die Leute gehen sollten. Diese Anordnung wurde in allen Zünften der Stadt verlautbart.

Dass die ergriffenen Maßnahmen auch gewisse Erfolge erzielen konnten, ist den zeitgenössischen Berichten zu entnehmen, die dabei auch aufschlussreiche Einblicke in die damalige Wahrnehmung von der Sterblichkeit und von der Wirksamkeit der Medizin in der Pestzeit vermitteln. Wilhelm Rem betonte nämlich in seiner Chronik ausdrücklich, dass in den städtischen Brechhäusern sehr viele Leute gesundeten, d.h. auf vier oder fünf Personen könne eine wieder genesen ${ }^{465}$. Die Augsburger Stadtärzte weisen in ihrem Traktat auch auf eine relativ niedrige Sterblichkeitsrate im städtischen Pesthospital hin, die vor allem dank der richtigen Anwendung der medizinischen Maßnahmen erreicht werden könne ${ }^{466}$. Auf die hohe Gesamtanzahl von 147 Insassen im Jahre 1535 entfielen dort wöchentlich nur 9 bis 16 Todesfälle. Außerdem wurde während der Pestepidemie von 1521 allgemein eine kleinere Sterblichkeit in der Stadt wahrgenommen. Nach der Schätzung Wilhelm Rems konnten damals ungewöhnlich viele Leute wieder zu ihrer Gesundheit gelangen: es wurden an allen

\footnotetext{
${ }^{465}$ StChr, Bd. 25, S. 163.

466 OCCO/SAYler/TIEFFENBACH/TALHAuSER, Was die Pestilentz an jr selbs sey [Augsburg: Philipp Ulhart 1535], E IIIv.
} 
orten viel leutt gesund, als nie mer gehört ist worden ${ }^{467}$. Abgesehen von der Subjektivität seiner Einschätzung kann man aber voraussetzen, dass sich die zahlreichen Maßnahmen des Rates auf den Verlauf der Epidemie in der Stadt positiv auswirkten.

Außer der Gründung der neuen Hospitaleinrichtung wurde 1521 noch das spezielle Personal vom Rat angestellt ${ }^{468}$. Zur medizinischen Versorgung der Pestkranken mit Aderlass und Heilmitteln waren fünf Barbiere in der Stadt tätig, die wöchentlich mit einem Gulden entlohnt wurden. Im Todesfall sollten ihre Familien vom Rat finanziell entschädigt werden ${ }^{469}$. Auch ernannte man spezielle Totengräber und - träger, die sich ausschließlich bei den Begräbnissen von Pestopfern betätigen ${ }^{470}$. Laut dem Befehl des Rates müssen sich diese Bediensteten allen Menschenversammlungen, Badestuben oder Wirtshäusern enthalten, um keine weitere Verbreitung der Seuche zu verursachen. Mit Pestopfern sollen sie bei Androhung einer Strafe ehrlich umgehen und sich insbesondere bemühen, die Leichen entsprechend tief in der Erde zu begraben. Zur besseren Kontrolle des Begräbniswesens wurde während der Pestzeit auch ein Aufseher verordnet, der speziell die Arbeit der Totengräber überwachen sollte.

\section{Die städtische Pestordnung von 1521}

Im Jahr 1521 erließ der Rat auch die ersten städtischen Anordnungen zu den Handlungsweisen in der Pestzeit, die bei Androhung von Geldstrafen von allen Stadtbewohnern beachtet werden mussten ${ }^{471}$. Diese Verordnungen waren direkt nach den dargestellten Vorschlägen der Stadtärzte entworfen. Zum Schutz vor der weiteren Verbreitung der Seuche wurden die Leute zunächst allgemein ermahnt, dass sie sich in der Pestzeit von unnötigen Versammlungen wie z.B. beim Tanzen fern halten sollen ${ }^{472}$. Insbesondere die genesenden Pestkranken durften noch vier Wochen lang nicht in die Badstuben oder Wirtshäuser gehen. Wenn sie gegen diesen Befehl des Rates verstießen, mussten sie einen

\footnotetext{
${ }^{467}$ StChr, Bd. 25, S. 163.

${ }^{468}$ StAA, CM IV, Nr. 13: Pestverordnungen des Rates (aus dem Inhalt datierbar für 1521).

${ }^{469}$ Für diesen medizinischen Dienst in der Stadt wurden die folgenden Barbiere angestellt: Hanns Trämel auf dem Beckenhaus, Ulrich Scheybenhart in Beuttingers Bader, Hans Heys im Schwarzenbad, Hans Gäspler im Blindenbad und Marx Blanck in Hansen Schmids Haus, in: StAA, Ratsprotokolle, 1521, Fol. 14. Die Badstuben sollten auf Begehr der Bader nur an drei Tagen in der Woche gehalten werden, und zwar bis Michaelis bis 6 Uhr am Nachmittag, und danach bis 5 Uhr am Nachmittag, in: StAA, Ratsprotokolle, 1521, Fol. 10.

${ }^{470}$ Der Totenträger sollte mit 6 Kreuzer Trägerlohn bezahlt werden, es sei denn jemand wollte freiwillig mehr zahlen, in: StAA, Ratsprotokolle, 1521, Fol. 12.

${ }^{471}$ StAA, CM IV, Nr. 13: Pestverordnungen des Rates (aus dem Inhalt datierbar für 1521).

472 Auch die gewöhnlichen Trinkfeste bei den Zünften anlässlich der Geburt eines Kindes von Zunftmeistern wurden in der Pestzeit nicht abgehalten, in: StChr, Bd. 25, S. 163.
} 
halben Gulden Strafe entrichten oder konnten aus der Stadt verwiesen werden, so lange sie das auferlegte Bußgeld nicht bezahlten. In diesen Verordnungen wurde jedoch kein allgemeines Stadtverbot für die Kranken oder Rekonvaleszenten verzeichnet, weshalb man voraussetzen kann, dass im Jahr 1521 noch keine strikte Isolierung von Pestkranken im Stadtraum eingeführt worden war. Diese Annahme scheint eine kurze Bemerkung in der Chronik von Wilhelm Rem zu bestätigen ${ }^{473}$. Laut seinem Bericht durften die Pestkranken zwar nicht in die allgemeinen Kirchen gehen, aber es wurde ihnen erlaubt, in zwei Kapellen die Messen zu hören: in der Minners Kapelle der Sankt Johannes Kirche und in der Bittschlins Kapelle der Sankt Ulrich Kirche.

Zur Vorbeugung der Infektionsgefahr wurde dagegen der Handel mit alter Kleidung, Betten und Hausrat auf allen Plätzen in Augsburg bei Strafe eines Guldens bis zum Widerruf des Rates untersagt. Sachen, die man aus einem derartigen Handel anschaffen konnte, stammten in dieser Zeit mutmaßlich vor allem aus den Hinterlassenschaften von Pestopfern. Nach der zeitgenössischen Erkenntnis konnte sich aus derartigen Gegenständen die giftige Materie weiter in der Stadt verbreiten, dem wollte die städtische Obrigkeit mit diesem Verbot vorbeugen. Bei derselben Bestrafung war auch das Aufhängen der Wäsche von Kranken auf den Strassen verboten.

Die städtische Obrigkeit wollte zugleich den Schrecken unter den Einwohnern während der Seuchenzeit möglichst einschränken, wodurch nach der damaligen Auffassung der Mediziner, die Erkrankung an der Pest leichter ihren Anfang nehmen könne. Aus diesem Grund verordneten die Ratsherren, dass den Verstorbenen, die damals zahlreicher als in den seuchenfreien Zeiten waren, nur am Samstag mit Glockenläuten gedacht wurde. Man solle auch die Anzahl der Pestopfer nicht öffentlich angeben. In diesem Zusammenhang ist auch das eingeführte Verbot des Tragens der das ganze Gesicht verhüllenden Trauermützen in der Pestzeit zu betrachten ${ }^{474}$. Nach der Erkenntnis des Rates können diese speziellen Kopfbedeckungen den Leuten nämlich mehr Entsetzen als Trost spenden.

Darüber hinaus wurden auch die Verordnungen zur Reinhaltung der Stadt erlassen, mit denen man sich vornehmlich vor der Luftvergiftung schützen wollte ${ }^{475}$. In Augsburg und in seinem Territorium wurde jetzt allgemein das Waschen in Röhrkästen und Schöpfbrunnen bei Strafe von 20 Kreuzer verboten. Die Leute sollten auch kein Spülwasser, das Abgeschöpfte vom Sauerkraut oder ähnliche Unsauberkeiten zu Hause behalten, sondern alles in die rechte Rinne auf den Strassen ausschütten. Dabei musste man aber beachten, dass das Wasser darin weiter

\footnotetext{
${ }^{473}$ StChr, Bd. 25, S. 163.

${ }^{474}$ SteTten, Geschichte, VIII. 13. 288; GASSER, Chronik, T. 3, S. 3; StChr, Bd. 25, S. 163.

${ }^{475}$ StAA, CM IV, Nr. 13: Pestverordnungen des Rates (aus dem Inhalt datierbar für 1521).
} 
floss. Auch wenn die Menschen vor ihren Häusern oder auf den Strassen einige Karren voll Mist, Asche, Müll oder anderen Unsauberkeiten stehen ließen, wollte der Rat sie je nach Ausmaß ihres Vergehens bestrafen. Dabei wurde insbesondere bei der Strafandrohung von einem Gulden das Schütten von Harn und Exkrementen auf die Gassen verboten, die man seither ausschließlich in den heimlichen Gemächern behalten oder in den Lech beseitigen solle.

Unter den Anweisungen zur Reinhaltung des Stadtraumes wurden auch obrigkeitliche Maßregeln für einige Handwerkergruppen erlassen, die vor allem durch unsachgemäßen Umgang mit Blut- und Fleischabfällen in ihrem Gewerbe leicht die Vergiftung der Luft verursachen können. Die Barbiere und Bader sollen das Blut der kranken und gesunden Menschen vom Aderlassen und Schröpfen nicht auf die Gassen in der Stadt schütten, sondern in die heimlichen Gemächer oder in den Lech tragen. Die Bader seien auch dazu verpflichtet, dass die kranken Leute nicht in ihre Badstuben eingelassen werden. Bei Nichtbeachtung dieser Befehle müssten sie einen halben Gulden bezahlen oder die Stadt verlassen, so lange das Bußgeld nicht entrichtet wurde. Der Rat ermahnte auch die Kürschner $^{476}$, Goldschläger ${ }^{477}$ und Saitenmacher ${ }^{478}$ dazu, dass sie die bei ihren Handwerksarbeiten entstandenen Fleisch- und Darmabfälle nicht auf die Gassen, bei der Strafe eines Guldens, schütten dürften. Die Färber sollen dagegen ihre verbrauchten Farben jetzt nicht mehr am Tag, sondern nur bei Nacht in den Lech beseitigen.

In der Pestzeit wurde auch die Schweinezucht unter strengere Kontrolle des Rates genommen. Die Bäcker ${ }^{479}$, Metzger und andere Leute, die Schweine in der Stadt hielten, durften die Futtertröge nicht mehr auf den Gassen aufstellen. Den Schweinemist und andere Unsauberkeiten musste man, so bald die Karre im Haus voll war, aus der Stadt in den Lech bei der Strafe von 20 Kreuzern - wegfahren. Die Kuttelwäscher ${ }^{480}$ sollten dagegen kein aus ihrer Gewerbetätigkeit übrig gebliebenes Blut, bei der Strafe eines halben Guldens, zum Füttern ihrer Schweine verwenden.

\footnotetext{
${ }^{476}$ Die Kürschner verarbeiteten in ihrem Handwerk Pelze, in: FISCHER, Schwäbisches Wörterbuch, Ebd., Bd. 4, Sp. 867: „Kürschner““.

${ }_{477}$ Goldschläger fertigten aus dem Gold dünne Blättchen. Dazu gebrauchten sie die feine Haut aus Rinder- oder Schafdärmen, in die das Gold zum Schlagen eingelegt wurde, in: GRIMM, Deutsches Wörterbuch: „Goldschläger“, „Goldschlägerhaut“.

478 Die Seitenmacher bearbeiteten vor allem das Leder aus den Seitenteilen von Tieren in: FISCHER, Schwäbisches Wörterbuch, Bd. 5, Sp. 1335-1336: „Seit“, „Seitenleder“.

${ }^{479}$ Eine wichtige Nebentätigkeit der Bäcker war die Schweinezucht, zu der vornehmlich die Abfälle ihres Gewerbes als Schweinefutter genutzt werden konnten, in: Ebd., Bd. 1, Sp. 744: „Becken-metzig“.

${ }^{480}$ Das Handwerk der Kuttelwäscher reinigte die Eingeweide von Tieren, in: Ebd., Bd. 4, Sp. 879: „Kuttelwäscher“.
} 
Der Rat verordnete auch die spezielle Aufzählung der Verstorbenen während der Pestepidemie $^{481}$. Clemens Sender berichtete nämlich in seiner Chronik, dass die Messner aus allen Augsburger Pfarren an jedem Freitag dem Ratsknecht auf den Zettel schreiben sollen, wie viele Leute innerhalb einer Woche gestorben $\operatorname{seien}^{482}$. Dabei wurden die Verstorbenen nicht nach den Todesursachen unterschieden, sondern man verzeichnete nur allgemein deren Gesamtanzahl. Laut den wöchentlichen Meldungen aus der Pfarre Sankt Ulrich starben seit dem 6. August 1521 bis zum 17. Januar 1522 insgesamt 649 Personen, wobei die höchsten Sterbezahlen auf die Monate September, Oktober und November entfielen ${ }^{483}$. Diese Todesanzeigen aus den Pfarren dienten während der Pestepidemie 1521/1522 als Vorlage für die Todeszählung für die gesamte Stadt, die in den Ratsprotokollen vermerkt wurde ${ }^{484}$. Nach ihren Angaben starben vom 25. Juli 1521 bis zum 9. März 1522 insgesamt 2.968 Menschen in Augsburg, davon 288 Personen in den neuen Brechhäusern ${ }^{485}$. In der Augsburger Bevölkerungstafel sind für die Jahre 1521 und 1522 entsprechend 3.895 und 1.980 Verstorbene verzeichnet ${ }^{486}$.

481 Das allgemeine Geburt-, Heirats- und Sterberegister in Augsburg wurde aber schon seit 1501 im Hochzeitsbuch verzeichnet, in: JAHN, Einwohnerzahl; RAJKAY, Bevölkerungsentwicklung.

${ }^{482}$ StChr, Bd. 23, S. 151.

${ }^{483}$ Nach den wöchentlichen Meldungen aus der Sankt Ulrichs Pfarre stellten sich dort die Todeszahlen folgendermaßen dar: 1. Woche (6. August 1521): 6 Menschen; 2. W: 8 M, 3.W: 11. M, 4. W: 10 M, 5. W: 15 M, 6. W: $30 \mathrm{M}, 7 . \mathrm{W}: 33 \mathrm{M}, 8 . \mathrm{W}: 43 \mathrm{M}, 9$. W: $53 \mathrm{M}, 10 . \mathrm{W}: 96 \mathrm{M}, 11 . \mathrm{W}: 78 \mathrm{M}, 12$. W: $77 \mathrm{M}, 13$. W (1. November): 41 M, 14. W: 47 M, 15. W: 48 M, 16. W: 29 M, 17. W: 26 M, 18. W: 15 M, 19. W: 24 M, 20. W: 18 M, 21. W: 17 M, 22. W (27. Dezember): 6 M, 23. W: 6 M, 24. W: 6 M, 25. W: 6 M.

${ }^{484}$ StAA, Ratsprotokolle, 1521, Fol. 12.

${ }^{485}$ In den Chroniken werden dagegen meistens die höheren Todeszahlen für diese Pestzeit angeführt. Abgesehen von den Chroniken von Wilhelm Rem und Paul von Stetten, die die Todeszahlen auch auf ca. 3.000 Personen schätzten, sind für etwa denselben Zeitraum in den anderen chronikalischen Aufzeichnungen 4.000 oder 5.000 Tote angegeben, in: Stetten, Chronik, VIII. 13.288; GASSER, Chronik, S. 3; StChr, Bd. 23, S. 152; Bd. 25, S. 163; SStBA, $2^{\circ}$ Cod. Aug. 71, Fol. 262v. Diese Todesstatistiken zeigen dabei das besondere Streben der Chronisten zur Hervorhebung der enormen Sterblichkeit in der Pestzeit, was man schon in den Chroniken aus dem Spätmittelalter beobachten kann, siehe S. 33.

${ }^{486}$ In den Jahren 1520 und 1523 starben dagegen in Augsburg 1.760 und 1.970 Menschen. 


\section{Ausbau der Pestbewältigung in Augsburg bis zur Mitte des 16. Jahrhunderts}

Seit dem Ende des 15. Jahrhunderts etablierten sich in Augsburg während der Epidemien die obrigkeitlichen Abwehrstrategien, denen die Anweisungen von gelehrten Medizinern zugrunde lagen. Die damals vom Rat ergriffenen Maßnahmen bildeten seither die Grundlage der städtischen Pestpolitik. Im Angesicht der Bedrohung durch eine Seuche griffen die Ratsherren auf die vorher erlassenen Verordnungen zurück, die danach nur in einigen Punkten modifiziert oder ergänzt wurden. Die weitere Entwicklung der städtischen Pestbewältigung bis zur Mitte des 16. Jahrhunderts wird im Folgenden ausführlich dargestellt.

\section{Anlegung des Oberen Gottesackers und Vorsorgemaßnahmen in den Jahren} 1533-1534

Im Vorfeld der nächsten Epidemie im Jahr 1535 wurden von der städtischen Obrigkeit bereits einige vorbeugende Maßnahmen ergriffen. In der Umgebung regierte die Seuche nämlich seit 1533, in deren Folge z.B. in Nürnberg vom Juli 1533 bis zum Februar 1534 insgesamt 5.754 Personen starben ${ }^{487}$. Angesichts der damals bestehenden Pestgefahr beschloss der Rat vor allem einen außerstädtischen Gottesacker anzulegen, um die Stadtgemeinde vor schädlichen Ausdünstungen aus den Gräbern zu schützen. Solche Pläne hatten die Ratsherren schon während der Pestepidemie von 1521, wie sie in ihrem Bittschreiben an Papst Leo X. formulierten $^{488}$. Dieses Konzept setzten sie endgültig während der Jahreswende von 1533/34 um, als der Obere Gottesacker vor dem Roten Tor (Abb. 20) angelegt wurde ${ }^{489}$. Zu diesem Zweck kaufte der Rat am 22. November 1533 der Priorin des Sankt Katharina Klosters einen Acker bei den Ziegelstadeln für 600 Gulden ab, zu dem man zusätzlich noch die daneben liegenden Gärten für die Summe von 500 Gulden erwarb. Auf dem neuen Friedhofsgelände, das mit einer Mauer umgeben wurde, erbaute man auch ein Haus für die Totengräber und eine Scheune für die dort nötigen Gerätschaften.

Obwohl die städtische Obrigkeit für ihre künftigen Gründungen der außerstädtischen Friedhöfe schon am 6. November 1521 eine generelle Bewilligung vom Papst erhalten hatte,

\footnotetext{
${ }^{487}$ PORZELT, Die Pest in Nürnberg, S. 38.

${ }^{488}$ Siehe S. 143-145.

${ }^{489}$ StChr, Bd. 23, S. 359; Bd. 29, S. 69; SStBA, $2^{\circ}$ Cod. Aug. 80, Fol. 115; GASSER, Chronik, T. 3, S. 27.
} 
traf diese Maßnahme auf heftigen Widerstand bei den Augsburger Kirchenherren. Für diese Reaktion scheinen dabei vor allem zwei Gründe verantwortlich zu sein.

Zum Ersten konnte die Anlage des neuen Gottesackers gemäß der päpstlichen Bulle nun selbst von den Ratsherren verordnet werden, weswegen sie ihre diesbezüglichen Beschlüsse anscheinend nicht mit den kirchlichen Machtträgern in Augsburg vereinbarten. Der neue Gottesacker sollte nämlich nach den Absichten des Rates zwei innerstädtische Kirchhöfe an der Sankt Moritz und der Sankt Ulrich Kirche ersetzen, wodurch deren Pfarrer offensichtlich mit der Beeinträchtigung ihrer Pfarrechte und der daraus fließenden Einkommen rechneten. Auf dem ersten Kirchhof herrschte bereits im Jahr 1494 ein Platzmangel für neue Begräbnisstätten, was schon damals eine wichtige Ursache für die Errichtung des Unteren Gottesackers $\operatorname{war}^{490}$. Die Verlegung des Sankt Moritz Kirchhofs führte aber zu heftigen Streitigkeiten mit den örtlichen Geistlichen, worüber Clemens Sender, ein Mönch von St. Ulrich und Afra in seiner Chronik berichtete ${ }^{491}$. Dieser Kirchhof wurde schon Ende 1533 völlig abgeschafft und der Rat ließ anschließend diesen Platz pflastern, um darauf zwölf Handelsstände zu bauen. Die Chorherren von Sankt Moritz wollten diesen Beschluss des Rates keinesfalls anerkennen, infolge dessen die städtischen Zechmeister ihnen diesen Platz mit gwalt nehmen mussten. Am 18. Dezember 1533 wurde dagegen auch ein obrigkeitliches Verbot für weitere Begräbnisse auf dem St. Ulrich Kirchhof erlassen, ungeachtet dessen, ob jemand darauf schon sein Grab angelegt hatte ${ }^{492}$. Nur die Personen, deren Grabstätten innerhalb der genannten Kirchen lagen, durften zukünftig noch dort begraben werden. Diese entschiedenen Verordnungen des Rates führten folglich dazu, dass die Augsburger Kirchherren den neuen Gottesacker nicht einweihen wollten: hat aber den gotzacker nit geweicht und die abgestorben leut, reich und arm, die nit aigne grebtnus in den kirchen haben, wie in ain ganrten als das vich begraben ${ }^{493}$.

Zum Zweiten hing der Streit um die Errichtung des neuen Gottesackers offensichtlich mit dem Beginn der Reformation in Augsburg zusammen, die seit 1533 von dem mehrheitlich protestantischen Rat schrittweise eingeführt wurde ${ }^{494}$. Die immer größere Einflussnahme weltlicher Obrigkeit auf Lebensbereiche, die früher von den geistlichen Machtträgern selbst geordnet oder wenigstens noch mitgeprägt werden konnten, erreichte ihren Höhepunkt am 17. Januar 1537 mit der offiziellen Einführung der Reformation in Augsburg. Die obrigkeitliche Beschränkung der Privilegien und Einkünfte von Pfarren bezüglich des Begräbniswesens

\footnotetext{
${ }^{490}$ Siehe S. 78-79.

${ }^{491}$ StChr, Bd. 23, S. 359.

${ }^{492}$ StAA, Ratsprotokolle, 1533, Fol. 87v.

493 StChr, Bd. 23, S. 359.

${ }^{494}$ Zur Einführung der Reformation in Augsburg, siehe S. 88-89.
} 
kann man in diesem Kontext auch als eine der ersten Verordnungen des Rates zur Unterdrückung der katholischen Kirche in der Stadt ansehen. Darüber hinaus wäre es auch möglich, dass die Anlegung dieses neuen Gottesackers von den katholischen Priestern als eine vom Reformationsgedanken geprägte Maßnahme wahrgenommen wurde. Obwohl sich die Idee der Auslagerung innerstädtischer Begräbnisstätten ursprünglich auf die Pestlehre gelehrter Medizinern zurückzuführen lässt, wurden diese medizinischen Schutzmaßnahmen seit der Drucklegung der theologischen Pestschrift von Luther 1527 auch mit der reformatorischen Bewegung assoziiert ${ }^{495}$. Durch seine eifrige Befürwortung der Friedhofsverlegungen wurde dieser medizinischen Anweisung von den Zeitgenossen unter Umständen auch ein konfessioneller Charakter zugeschrieben. Wie die Kontroversen um die neue Begräbnisordnung von 1536 in Leipzig anschaulich zeigen, konnten die katholischen Theologen eine direkte Verbindung zwischen den außerstädtischen Friedhöfen und dem Luthertum herstellen $^{496}$. Nach ihrer Deutung stellten nämlich die Begräbnisse außerhalb der Stadt einen verdeckten Angriff auf jene alltäglichen Praktiken der Katholiken dar, die die Lebenden und die Toten $\mathrm{zu}$ einer ewigen Gemeinschaft verband. Derartige Begräbnispraktiken bedeuteten das Ende eines religiösen Konzeptes, nach dem die „Gegenwart der Toten“ als ein wichtiger Teil der christlichen Gemeinde gedeutet wird ${ }^{497}$. In der lutherischen Doktrin wurde dagegen die Vorstellung der Fürsprache für die Toten durch die Lebenden und die Ausübung der dazugehörigen religiösen Praktiken streng abgelehnt, weshalb das Verbot der innerstädtischen Friedhöfe mit der theologisch begründeten Trennung der Lebenden und der Toten verknüpft werden könne.

Die Gründung des Oberen Gottesackers wurde trotz des heftigen Widerstandes der Kirchenherren endgültig umgesetzt, wobei seine Einweihung offensichtlich nicht vom Bischof oder einem anderen hohen katholischen Würdenträger in Augsburg, sondern vermutlich nur von einem der Reformation zugeneigten Priester oder Mönch durchgeführt werden konnte. Am 18. Februar 1534 wurde Zimprecht Hoffer, Ratsmitglied und Baumeister in Augsburg als erster Toter dort begraben ${ }^{498}$. Die Auswahl dieses außerstädtischen Gottesackers als Begräbnisstätte für einen Ratsherrn zeigt die große Unterstützung des Rates

\footnotetext{
495 Siehe S. 112.

${ }^{496}$ Zum Streit um die Begräbnisordnung von 1536 in Leipzig und zum Thema der Verlegung von Friedhöfen im deutschsprachigen Raum: KoSLOFSKY, The reformation of the Dead, S. 17-78; DERS., >Pest< - >Gift - >Ketzerei<; DERS., Die Trennung der Lebenden von den Toten.

${ }^{497} \mathrm{Zu}$ diesem religiösen Konzept im Mittelalter in: OEXLE, Die Gegenwart der Toten.

${ }^{498}$ SStBA, $2^{\circ}$ Cod. Aug. 71, Fol. 313v.
} 
für die neue Friedhofsanlage, was auch in Leipzig von den protestantischen Ratsmitgliedern und Bürgermeistern öffentlich getan wurde ${ }^{499}$.

Weitere Vorsorgemaßnahmen wurden vom Rat am 14. April 1534 getroffen $^{500}$. Mit ihnen wollte man sich sowohl vor der Übertragung der Infektion von außen schützen als auch auf die mögliche Epidemie vorbereiten. Angesichts der ständigen Seuchenbedrohung befahlen die Ratsherren, dass die Torwärter keine auswärtigen Kranken in die Stadt einlassen dürften. Dasselbe galt für die mit altem Hausrat hausierenden Leute, die damals nur mit Erlaubnis des Rates oder der Bürgermeister in die Stadt hineingelangen konnten. Auch die Stadtbewohner dürften nur dann Fremde in ihre Häuser aufnehmen, wenn die städtischen Behörden dazu eine entsprechende Genehmigung erteilten. Mit diesen Maßnahmen wolle man die Stadtgemeinde vor der Seuche schützen, die zusammen mit Flüchtlingen und deren Sachen aus anderen infizierten Orten nach Augsburg eingeschleppt werden könnte.

Trotz der erlassenen Verordnungen wollten sich jedoch auch die Stadtherren auf den möglichen Seuchenausbruch vorbereiten, worin sich die zeitgenössische Vorstellung der Pest als einer wiederkehrenden Plage deutlich widerspiegelt. Der Rat beriet ganz im Allgemeinen darüber, welche Vorkehrungen, z.B. welche zusätzlichen Anstellungen, für die Bewältigung der Pest getroffen werden könnten. In der Stadt plante man bis zu acht Barbieren fest zu besolden, damit sie ständig in Augsburg verblieben und zu den Kranken gingen. Die Stadtärzte sollten jetzt besondere Vorsorge bei der Aufwartung der Kranken in der Stadt walten lassen. Für die Organisation des städtischen Begräbniswesens beschloss der Rat dagegen, künftig eine spezielle Ordnung festzulegen. Außerdem wollte man noch zukünftig genau bestimmen, welches Personal in den Brechhäusern anzustellen sei. In diesem Zusammenhang unterstrichen die Ratsherren, dass nur diejenigen Pestkranken, welche sich in die städtische Einrichtung des Brechhauses begeben bzw. einweisen lassen, ihren Lebensunterhalt von der Stadt Augsburg finanziert bekämen. Damit wolle man bewirken, dass mehr Leute in die Brechhäusern aufgenommen und dadurch strikter von den Gesunden isoliert werden könnten. Demselben Zweck sollte ursprünglich eine andere Verordnung dienen, die jedoch aufgegeben wurde. In diesem gestrichenen Punkt wollte der Rat nämlich befehlen, dass, falls ein Kranker nicht in den städtischen Brechhäusern versorgt werden wolle, sein Haus für die Krankheitsdauer und noch vier Wochen nach der Genesung zugesperrt werden müsste. Ein solches Gebäude dürfte jeweils nur eine Person verlassen, um die Lebensmittel für alle Hausbewohner einzukaufen.

\footnotetext{
${ }^{499}$ KOSLOFSKY, The reformation of the Dead, S. 74.

${ }^{500}$ StAA, CM IV, Nr. 13: Ratsverordnungen wegen der Pestgefahr (1534, 14. April).
} 


\section{Ausbruch der Epidemie und die städtische Pestordnung von 1535}

Die in den anderen Städten herrschende Epidemie erreichte im Sommer 1535 auch Augsburg, wo sie nach den Angaben in der Chronik von Sender am 4. Juli begann ${ }^{501}$. Ihr Höhepunkt fiel dabei auf die Woche um den 29. September 1535, als in der Stadt insgesamt 118 Personen starben. Danach ließen die Mortalitätsauswirkungen der Seuche deutlich nach und seit dem 5. März 1536 gab es keine Pestopfer mehr in Augsburg. Diesmal war der Verlauf der Pestepidemie nicht so tödlich wie in den früheren Jahren gewesen, so dass nach den Angaben von Sender in dieser Zeitperiode insgesamt 2.327 Menschen in der Stadt starben ${ }^{502}$.

Die Tätigkeit des Stadtregiments in der Pestzeit wurde genau wie im Jahr 1521 geregelt, indem ein dritter Teil aller Ratsmitglieder und nur die drei wichtigsten Ratsämter: Baumeister, Einnehmer und Ungelter seit August 1535 zusammenkamen und die Amtsgeschäfte verwalteten. Die Einschreibung der Steuer wurde erneut eingestellt und erst wieder am 17. Januar 1536 von den Steuerherren durchgeführt ${ }^{503}$. Auch diesmal wurden die umfangreichen Verordnungen zur städtischen Pestbewältigung vom Rat erlassen, die direkt an den in den Jahren 1521/22 ausgearbeiteten Maßnahmenkatalog anknüpften. In diesen obrigkeitlichen Handlungen ist dabei ein vermehrtes Streben nach Intensivierung der früheren Anordnungen zu erkennen, die sowohl die spezielle Gesetzgebung wie auch die Anstellung des städtischen Personals für die Pestzeit betrafen.

Am 12. August 1535 erließ der Rat eine städtische Pestordnung, die mit Hilfe gedruckter Anschläge in Augsburg verkündet wurde ${ }^{504}$. Diese obrigkeitlichen Anordnungen für die Pestzeit basierten auf den entsprechenden Regelungen aus der Zeit der Epidemie von 1521, die nun jedoch erweitert und ergänzt wurden. Eine detaillierte Analyse dieses Gesetztextes lässt die wichtigsten Grundzüge des damaligen Pestkonzeptes erkennen, das grundsätzlich durch ein Zusammenspiel des religiösen und medizinischen Diskurses konstruiert wurde.

\footnotetext{
${ }^{501}$ StChr, Bd. 23, S. 396; Auch in: GASSER, Chronik, T. 3, S. 29; STETTEN, Geschichte, VIII. 20. 338.

${ }^{502}$ In einer anonymen Chronik aus dem 17. Jahrhundert wurde die Anzahl von ca. 1.540 Verstorbenen während dieses Ausbruchs der Pest verzeichnet: SStBA, $2^{\circ}$ Cod. Aug. 53; Laut der Bevölkerungstafel starben in den Jahren 1535-36 entsprechend 1.482 und 1.492 Personen, was im Vergleich zu den pestfreien Jahren 1534 mit 1.282 und 1537 mit 1.462 keinen beträchtlichen Unterschied in der Mortalität in Augsburg ausmacht. In den gedruckten Ausgaben der Bevölkerungstafel seit dem 17. Jahrhundert wurde zwar unter dem Jahr 1535 die Zahl von 13.000 Verstorbenen markiert, die sich jedoch nach dem Überprüfen mit den handschriftlichen Einträgen im städtischen Hochzeitsbuch und dem ersten Einblattdruck mit diesen vitalstatistischen Daten als ein Druckfehler entblößte: Augsburger Bevölkerungstafel, Nr. 142-144, 347, SStBA, $2^{\circ}$ Lw Einblattdrucke nach 1500; Dazu auch in: JAHN, Einwohnerzahl, S. 391; RAJKAY, Bevölkerungsentwicklung, S. 257.

${ }^{503}$ StAA, Ratsprotokolle, 1535: Fol. 105-106v, 107v; 1536: Fol. 108v.

${ }^{504}$ Augsburger gedruckte Pestordnung vom 12. August 1535, SStBA, $2^{\circ} \mathrm{S} 14$, Kapsel 1, Nr. 21; Der handschriftliche Entwurf neben anderen Verordnungen für die Pestzeit wurde dagegen verzeichnet, in: StAA, CM IV, Nr. 13: Pestverordnungen des Rates (1535).
} 
In der Einleitung der städtischen Pestordnung wird zunächst eine religiöse Auslegung der ausgebrochenen Pestepidemie dargestellt. Der zeitgenössischen Erkenntnis zufolge lag der Ursprung der Seuche im Verhängnis Gottes, der die Stadt Augsburg und einige andere Gebiete Oberdeutschlands mit der Rut der Sucht des Brechens bestrafte. Diese göttliche Strafe entspränge aus den menschlichen Missetaten, die auch von den Menschen in Augsburg alläglich begangen werden. Daher wolle der Rat zunächst allen Stadtbewohnern daran erinnern und sie dazu ermahnen, dass ain yeder/ zuvorderst/ den übernatürlichen Artzt/ Christum/ unnsern Hailand/ der allain alle Kranckhaiten gewißlich erkennen/ auch an Seel und leib hailen kan, anruflich ersuchen wolle. Mit seiner Strafe riefe Gott nämlich alle Menschen in Trübsal, Krankheit oder Furcht an, dass sie sich wieder zu ihm bekehren sollen. Allen Leidenden, die sich mit warer rew/ pußwirckung/glauben/lieb/ und hoffnung an ihn wandten, verheiße Gott nicht nur Heilung sondern auch beständige Gesunderhaltung. In diesen Ausführungen ist auch die Einflussnahme der sich in Augsburg durchsetzenden Reformation zu erkennen, weil im Mittelpunkt der religiösen Praktiken nur Christus als einziger Fürsprecher in der Pestzeit genannt wurde ${ }^{505}$.

Der weitere Inhalt der städtischen Pestordnung ist jedoch ausschließlich vom medizinischen Diskurs geprägt, nach dem alle nachfolgenden Anordnungen des Rates formuliert wurden. Im Vergleich zu den ersten Maßregeln von 1521 stand nun die Infektionsvorbeugung im städtischen Raum in Vordergrund, wozu schon im ersten Teil des Raterlasses umfangreiche Regelungen angeführt waren. Gleich nach der religiös motivierten Einleitung wies der Rat nämlich daraufhin, dass diese Krankheit beflecklich war, wodurch sich ein Mensch leicht von einem anderen vergiften könne. Damit man sich aber nicht gegenseitig schadete, sollten die Menschen unbedingt dess hoechsten schatz der vernunft/ inen allain vor andern Creaturn gnedigklich mitgetailt/ gebrauchen. Deshalb wolle der Rat spezielle Verordnungen für die Pestzeit vornehmen, die dem Wohl der ganzen Stadtgemeinde dienen sollen.

Nach der Erkenntnis des Rates könnten die Kranken von den Gesunden in solher haimsuchung Gottes nicht sofort unterschieden werden, weil die eindeutigen Krankheitszeichen üblicherweise erst später ausbrechen. Ein infizierter Mensch könne daher viele gesunde Menschen beflecken, bevor er von ihnen abgesondert wurde. Aus diesem Grund befahl der Rat sich von unnötigen Menschenansammlungen, insbesondere beim Tanzen fernzuhalten und diese ausdrücklich zu meiden. Auch die Besuche im Rathaus solle man in der Pestzeit auf das Notwendige beschränken, um die Stadtherren vor der Ansteckungsgefahr zu schützen. Daher dürften sich die Menschen nur dann zum Rat, Gericht, zu den

\footnotetext{
${ }^{505}$ Siehe S. 87-95.
} 
Steuerherren, Baumeistern oder anderen städtischen Behörden begeben, wenn sie eine bedeutsame Angelegenheit zu verhandeln hätten. Um der weiteren Verbreitung der Infektion wirksamer entgegenzutreten, richtete der Rat einen direkten Appell an alle Hausväter. Sie sollten nämlich ihre Familien und Hausgenossen dazu ermahnen, dass sie sich weder zu den Pestkranken selbst, in deren Häuser oder an andere Orte begaben, noch zu Begräbnissen gingen. Stattdessen solle jeder Einwohner sich und andere vor dieser Gefahr möglichst beschützen. Die Bürger oder Einwohner Augsburgs dürften auch keine mit der Pest befleckten Personen, ungeachtet dessen, wie nah sie mit ihnen verwandt oder befreundet seien, in ihre Häuser oder irgendwo anders in der Stadt aufnehmen. Im Gegensatz zu den städtischen Regelungen von 1521 wurde jetzt auch eine völlige Quarantäne der Kranken und Rekonvaleszenten eingeführt. In dieser Pestordnung befahl der Rat, dass alle Pestkranken, denen Gott zur Erlangung ihrer Gesundheit aufhülfe, sich noch vier Wochen danach in ihren Häusern aufhalten müssten und unter keinem Vorwand herauskommen dürften. Wenn aber jemand gegen dieses obrigkeitliche Verbot verstoße, müsse er sofort aus der Stadt ausgewiesen werden. Dagegen beschlossen die Ratsherren keine vollständige Quarantäne der Menschen, die die Pestkranken in ihren Häusern beherbergten ${ }^{506}$. Die Personen aus infizierten Haushalten wurden lediglich dazu ermahnt, dass sie während der Krankheitsdauer ihrer Mitbewohner möglichst wenig unter die Leute gingen. Dabei sollen sie sich insbesondere der Besuche beim Rathaus, bei der Ratskanzlei und bei den Zunfthäusern sowie bei anderen öffentlichen Orten im höchsten Maße enthalten. Bei Übertretung dieses Verbots verhänge der Rat eine zu dem begangenen Vergehen entsprechende Strafe. Auch die Badestuben dürften die Pestkranken und ihre Mitbewohner nicht besuchen, weshalb die Bäder fleißig kontrollieren werden sollen. Für einen Verstoß gegen dieses Verbot drohe der sofortige Verweis aus der Stadt. Die anderen Punkte der Verordnung zur Infektionsvorbeugung betrafen den Umgang mit Gegenständen aus Gewandstoff, über die sich nach Ansicht der medizinischen Pestlehre die giftige Materie weiter verbreiten könne. Unter den diesbezüglichen Maßnahmen wurden erneut Regelungen aus dem Jahr 1521 angeordnet, wie das Verbot des Handels mit alter Kleidung, mit Betten und Hausrat auf allen Plätzen in Augsburg und das Verbot des Aufhängens und Waschens von Kleidung und Bettzeug von Kranken und Rekonvaleszenten auf den Straßen. Zum Waschen, Aufhängen und Trocknen solcher Gegenständen aus Gewandstoff bestimmte der Rat nun einige Orte außerhalb der Stadt: an der Sinckalt, unter der Hertgassen und bei den Hütten neben der neuen Mühle.

\footnotetext{
506 Eine weitgehende Quarantäne der Mitbewohner aus den infizierten Häusern wurde zwar in den vorhergehenden Ratsberatungen erwogen, endgültig aber aus den vorzunehmenden Verordnungen gestrichen, in: StAA, CM IV, Nr. 13: Ratsverordnungen wegen der Pestgefahr (1534, 14. April). Dazu siehe S. 155.
} 
Außerdem wurde auch ein allgemeines Verbot des Ausklopfens und Sonnens von Gewändern der Kranken und Gesunden auf Straßen, Kirchhöfen, Kreutzgärten oder Höfen bei der Bestrafung eines Guldens erlassen.

Die übrigen Regelungen in dieser Pestordnung betrafen grundsätzlich die Reinhaltung der Stadt, welche bis auf wenige Veränderungen mit denjenigen von 1521 übereinstimmten ${ }^{507}$. Bezüglich des Waschverbots in der Stadt erlaubte der Rat nun die Sachen von gesunden Wöchnerinnen und ihren Kindern in Röhrkästen und Schöpfbrunnen zu waschen. Die Strafe für unerlaubtes Ausschütten von Fleisch- und Darmabfällen bei den Gewerken der Kürschner und Saitenmacher wurde zudem von 20 auf 15 Kreuzer gemildert ${ }^{508}$. In der städtischen Pestordnung wurde auch die neue Regelung der Begräbnisse verzeichnet, die während der Epidemie ausschließlich auf zwei Gottesäckern stattfinden sollte. Die Schweinezucht in Augsburg wurde dagegen unter strengere Kontrolle des Rates gestellt. Unter Androhung der Geldstrafe von 20 Kreuzern bei Zuwiderhandlung, dürften die Schweine nun noch einmal täglich, d.h. am frühen Morgen, zur Schwemme getrieben werden. Anschließend müssten sie sofort wieder in die Stadt zurückgetrieben werden. Auch bezüglich der städtischen Rinderzucht verordnete man, dass der Kuhharn nach dem morgendlichen Hornblasen in den Lech getragen werden müsse. Ein Verstoß dagegen solle mit einem Gulden geahndet werden. In einem anderen Ratsbeschluss, der in diese gedruckte Pestordnung nicht aufgenommen wurde, sondern den man direkt den Betroffenen mitteilte, erklärte man, dass die Bäcker und die Bierschenken während der Pestzeit kein Wasser zum Backen und Sieden aus dem Lech schöpfen sollten ${ }^{509}$. Dieses Verbot steht dabei in engem Zusammenhang mit jenen obrigkeitlichen Anordnungen, die das Ausschütten von Unrat und Abfällen gerade in diesen Fluss geboten. In der damaligen Wahrnehmung konnte dadurch das Wasser des Lechs auch mit der Pest vergiftet worden sein.

Am Ende der städtischen Pestordnung stellt der Rat fest, dass im Verlauf der Epidemie noch andere Ordnungen erlassen und die dazu verordneten Personen angestellt werden müssten. Mit diesem umfangreichen Maßnahmenkatalog erhofften die Stadtherren bewirken zu können, dass solche vor augen schwebende Sucht/ dardurch vermittelst Goetlicher hilff/ darein wir fürnemlich/ all unser vertrawen setzen/ nit wenig geringert werden solle. Dabei wolle der Rat je nach Bedarf die erlassenen Verordnungen künftig erweitern und verbessern.

\footnotetext{
${ }^{507}$ Siehe S. 150-151.

${ }^{508}$ Die Goldschläger wurde in dieser städtischen Pestordnung nicht mehr erwähnt.

509 StAA, CM IV, Nr. 13: Pestverordnungen des Rates (1535); StAA, Schätze, Nr. 16, p. 8R-11: Pestverordnungen des Rates pro Memoria (1535).
} 


\section{Die Begräbnisordnung von 1535 und andere Anordnungen zur Eindämmung der Seuche}

Nach der Verlautbarung der städtischen Pestordnung unternahmen die Ratsherren noch weitere Maßnahmen, mit denen die Verbreitung der Seuche in der Stadt verhindert werden sollte. Zum wirksameren Schutz der Stadtgemeinde vor den schädlichen Ausdünstungen aus den Gräbern wurde im Jahr 1535 vom Rat eine spezielle Begräbnisordnung festgelegt ${ }^{510}$. Aufgrund des obrigkeitlichen Verbotes durften seither generell alle Verstorbenen nicht mehr auf den Kirchhöfen oder in den Kapellen innerhalb der Stadt begraben werden, ungeachtet dessen, ob jemand schon dort sein Grab hatte. Demgemäß sollte das gesamte Begräbniswesen während der Epidemie in Augsburg ausschließlich auf zwei städtischen Gottesäckern organisiert werden: für die St. Ulrich und die St. Moritz Pfarrei auf dem neuen Oberen Gottesacker vor dem Roten Tor und für die übrigen Sprengel auf dem Unteren Gottesacker hinter der St. Stephan Kirche. Zur Ausführung der Begräbnisse wurden von der Stadt acht Totenträger und vier Totengräber angestellt, deren Tätigkeit zwei Aufseher (fissner) kontrollierten. Diese Bediensteten hatten sich Tag und Nacht an zwei bestimmten Orten aufzuhalten, damit die Stadtbewohner sie im Notfall sofort aufsuchen konnten. Die erste Gruppe der städtischen Verordneten vom Oberen Gottesacker sollte ständig in einer Hütte (Borhitten) auf dem St. Ulrich Kirchhof anwesend sein, während sich die zweite Gruppe vom Unteren Gottesacker in der St. Gallus Kapelle aufhielt. Für ihren alltäglichen Dienst erhielten sie wöchentlich einen Gulden.

Nach den Anleitungen der Begräbnisordnung sollten alle am Tag verstorbenen Menschen sofort aus ihren Häusern auf den festgelegten Gottesacker hinausgetragen werden. Dagegen wurden die Toten in der Nacht zunächst in speziellen Hütten innerhalb der Stadt aufbewahrt, um gleich nach der Morgendämmerung auf den Gottesäckern begraben zu werden. Die verordneten Aufseher sollten diese Hütten abends und morgens fleißig inspizieren, damit die Leichen daraus immer rechtzeitig bestattet würden. Auch während der Begräbnisse müssten sie immer darauf achten, dass die Toten ausreichend tief begraben wurden, damit daraus keine schädlichen Ausdünstungen aufsteigen könnten. Zunächst müsse man die Leichen mit einer Schicht Kalk und Erde bedecken und danach das Grab zusätzlich mit aufgeweichter Erde zuschütten.

\footnotetext{
510 StAA, CM IV, Nr. 13: Begräbnis- und Pestverordnungen des Rates (1535); StAA, CM IV, Nr. 13: Pestverordnungen des Rates (1535); StAA, Schätze, Nr. 16, p. 8R-11: Pestverordnungen des Rates pro Memoria (1535).
} 
Das Verbot der innerstädtischen Begräbnisse galt für alle Kirchhöfe und Grüfte in den Kirchen, von dem man nur mit einer speziellen Erlaubnis des Rates befreit werden konnte. Davon ausgenommen waren nur die einflussreichsten Bürger in der Stadt. Als am 3. Dezember 1535 Herr Raymund Fugger starb, hatte seine Familie eine spezielle Einwilligung der Ratsherren erworben, um ihn in der Grabkapelle der Fuggers in der St. Anna Kirche begraben zu dürfen. Dabei bemühten sich die Fugger möglichen Protesten unter der Stadtbevölkerung aufgrund dieser Ausnahme vorzubeugen, indem man den Sarg ohne die üblichen Begräbnisfeierlichkeiten durch den Garten in die Fuggerkapelle hineingetragen hatte $^{511}$. Auch Herr Laux Lang, dessen Familie in engen Beziehungen mit dem Herzog Georg von Bayern-Landshut stand, konnte nach seinem Tod am 15. Dezember 1535 ausnahmsweise in der Kapelle auf dem Fronhof innerhalb der Stadt begraben werden ${ }^{512}$.

Im Ratsdekret vom 4. November 1535 wurde auch der Schweinehandel speziell geregelt ${ }^{513}$. Der Rat hatte bemerkt, dass viele Schweine nach Augsburg zum Verkauf getrieben wurden, und zwar aus den Orten, in denen zurzeit das Schweinesterben umging. Da man glaubte, dass daraus, insbesondere während der gegenwärtigen Pestepidemie, viel Unrat entspringen könne, beschloss der Rat der weiteren Vergiftung des städtischen Raumes entschieden entgegenzuwirken. Laut seinem Befehl mussten alle in Augsburg ankommenden Händler eine amtliche Urkunde vorlegen, dass ihre Tiere nicht aus Orten hierher getrieben worden seien, wo zu diesem Zeitpunkt eine Schweinepidemie herrschte. Zudem war ein derartiger Handel ausschließlich auf dem städtischen Saumarkt vor der St. Jakobs Kirche gestattet, damit die Schweine nicht schon vor den Stadttoren ohne obrigkeitliche Kontrolle verkauft wurden. Während einer erneuten Schweineseuche 1537 in der Umgebung Augsburgs wurde dasselbe Ratsdekret mit der Begründung erlassen, dass unreines und fauliges Schweinefleisch für die Menschen im höchsten Masse schädlich sei ${ }^{514}$. In den Jahren 1541 und 1544 erließ der Rat weitere Verordnungen betreffs der Schweinezucht in Augsburg, die eindeutig als Vorsorgemaßnahme gegen die Seuchen anzusehen $\operatorname{sind}^{515}$. Nach der Erkenntnis der Ratsherren erhoben sich in der Stadt viel Gestank und schädliche Ausdünstungen wegen der unsachgemäßen und unsauberen Schweinehaltung, was sowohl die hiesigen Bürgern als auch ehrenwerte Gäste mit Missfallen wahrnahmen. Derartige Unordnung könne nicht nur der Stadt einen schlechten Ruf einbringen, sondern trüge auch merklich zur Vergiftung der Luft

\footnotetext{
${ }^{511}$ StChr, Bd. 29, S. 69-70; dazu vgl. auch PöLnITZ, Anton Fugger, S. 347-348.

${ }^{512}$ StChr, Bd. 29, S. 70.

${ }^{513}$ StAA, Schätze, Nr. 16, p. 16: Schweinkauffs fursehung im Sawsterbend (4. November 1535).

${ }^{514}$ StAA, Schätze, Nr. 16, p. 30R-31: Schweine betreffend (8. November 1537).

${ }^{515}$ StAA, Schätze, Nr. 16, p. 64-64R: Das umbtreiben der Schwein betreffend (14. Mai 1541; 23. November 1544).
} 
im Stadtraum bei. Aus diesen Gründen befahl der Rat, dass die Schweine möglichst schnell zur nächsten Schwemme und wieder zurück getrieben werden sollen. Beim Treiben der Schweine auf die Äcker habe man die Gassen zu meiden und solle die Tiere möglichst beim Stadtgraben zum nächsten Tor hinausführen. Das Herumlaufen der Schweine bei Tag und Nacht auf den Gassen und insbesondere am Flussufer wurde seither ebenfalls vom Rat bestraft.

Nach dem Ausklingen der Epidemie erließ der Rat im März 1536 eine Anordnung, die auch im engen Zusammenhang mit der städtischen Pestpolitik steht ${ }^{516}$. Angesichts der geschwinden schweren leuff, womit offensichtlich die vergangene Seuche gemeint ist, solle der gesamte Handel mit Kleidung, Lebensmitteln und anderen Gegenständen nur noch auf dem Trendelmarkt und einigen wenigen anderen Handelsplätzen oder Läden in Augsburg stattfinden. Das Hausieren mit solchen Gegenständen, die in dieser Zeit noch aus den Häusern der Pestopfer stammen könnten, sei wegen der möglichen Infektionsgefahr vollständig verboten.

\section{Erweiterung des städtischen Dienstpersonals in der Pestzeit 1535}

Zur Umsetzung der städtischen Maßnahmen wurden 1535 zahlreiche neue Bedienstete vom Rat abgeordnet. Darin ist eindeutig eine allgemeine Tendenz zur Erweiterung des Dienstpersonals für die Zeit der Pest zu erkennen ${ }^{517}$. Zum Transport der Kranken in die Brechhäuser waren vier Sesselträger ernannt, die die kranken Leute auf speziellen Tragbahren durch die im Umfeld am wenigsten bewohnten Tore hinaustragen sollten. Diese Dienstleute mussten sich dabei zusammen mit den Totenträgern und Totengräbern an den zwei oben genannten Orten ständig zur Verfügung halten, um Tag und Nacht für die Stadtbewohner auffindbar zu sein. Außerdem verordnete der Rat sechs Männer und acht Frauen, die den Kranken in der Stadt aufwarten und ihnen religiösen Trost und Zuspruch spenden sollten. Wenn jemand an der Pest gestorben war, halfen diese Pflegerinnen auch noch bei den Vorbereitungen zum Begräbnis, indem sie die Leichen in spezielle Säcke einnähten. Namen und Wohnorte dieser Bediensteten waren in der Stadt öffentlich verlautbart worden. Für ihren Dienst erhielten die Pfleger und Sesselträger jeweils einen Gulden wöchentlich. Die

\footnotetext{
${ }^{516}$ StAA, Schätze, Nr. 16, p. 21-21R: Abschaffung der hausierung und der verhinderung der Oberkait Beuelhs den Petelknechten wider die Straifennden Petler gegebenn (18. Juni 1541).

517 StAA, CM IV, Nr. 13: Begräbnis- und Pestverordnungen des Rates (1535); StAA, CM IV, Nr. 13: Pestverordnungen des Rates (1535); StAA, Schätze, Nr. 16, p. 8R-11: Pestverordnungen des Rates pro Memoria (1535).
} 
Reinhaltung des Stadtraums bewachte der Rat dagegen mit Hilfe von Gassenhauptleuten, die besonders beachten sollten, dass die Stadtbewohner vor ihren Häusern oder auf den Gassen keinen Unrat liegen ließen ${ }^{518}$. Alle Verstöße gegen die städtischen Hygieneverordnungen mussten sie ihren Viertelhauptleuten melden, die die Strafe nach dem Ausmaß der Übertretungen verhängten.

Zur medizinischen Versorgung der Kranken in der Stadt wollte der Rat ursprünglich sechs Barbiere anstellen, von denen mutmaßlich wegen des milderen Verlaufs der Epidemie letztlich nur drei angenommen wurden ${ }^{519}$. Ihre Behausungen sollten mit roten Fahnen markiert werden. Die Ratsherren beabsichtigten auch eine spezielle Apothekerordnung zu erlassen, deren Bestimmungen aber nicht überliefert sind. Diese Apothekerordnung sollte mutmaßlich der Versorgung der Stadtbewohner mit preiswerten Arzneimitteln dienen. Auch die vier Stadtärzte waren aufgrund ihrer Dienstverträge zur Behandlung der Pestkranken verpflichtet. Der Rat wandte sich dabei an diese eingeschworenen Doktoren mit der Anweisung, dass sie für den gemeinen Mann noch eine deutschsprachige Druckschrift über die Pestilenz zusammenstellen sollten. Dass diese obrigkeitliche Aufforderung von ihnen bald ausgeführt wurde, bezeugt die schon erläuterte Pestschrift aus dem Jahr $1535^{520}$.

Von den vier städtischen Doktoren sollten ursprünglich auch zwei Doktoren zur medizinischen Behandlung und Aufnahmekontrolle in die Brechhäuser verordnet werden ${ }^{521}$. Schließlich beschloss der Rat noch einen weiteren gelehrten Arzt für die Zeit der Pest zu besolden, der ausschließlich zur Versorgung dieser städtischen Einrichtung angestellt wurde. Für diesen Dienst ernannte man Doktor Bernhardin Schludin (gest. 1566), der den Rat um ein spezielles Gehalt in der Pestzeit ersuchte ${ }^{522}$. Auf sein Bittschreiben war auch die Idee der Ratsherren für eine befristete Anstellung eines speziellen Pestarztes zurückzuführen ${ }^{523}$.

\footnotetext{
518 Die Gassenhauptleute, die auch Hauptleute über zehn Häuser genannt waren, bildeten eine Verbindungsinstitution zwischen dem Rat und der Bürgerschaft. Die Bewohner aus bestimmten Häusern hatten sich unter ihrem Kommando z.B. beim Läuten der Sturmglocke bei Feuer oder Kriegsgefahr vor den Stadttoren oder auf den großen Plätzen zu versammeln. Die Gassenhauptleute unterstanden dabei in den acht Stadtvierteln jeweils zwei Viertelhauptleuten, die vornehmlich aus dem Kleinen Rat ernannt wurden. Seit der Mitte des 15. Jahrhunderts spielte die territoriale Organisation der Bürgerschaft unter den Gassenhauptleuten eine immer wichtigere Rolle bei der Durchführung von militärischen und administrativen Maßnahmen des Rates. Dazu in: RoGGE, Für den Gemeinen Nutzen, S. 142-150.

${ }^{519}$ Dazu wurden folgende Barbiere angestellt: Georg Grapmair, in der Breuerin Hof - Plindenbader, Hanns Ernst, Poggenburger Bader - Plindenbader und Hanns Pärtlin, Beckenbader - Leupolds Bader, in: StAA, Schätze, Nr. 16, p. 8R-11: Pestverordnungen des Rates pro Memoria (1535).

${ }^{520}$ OCCO/SAYLER/TIEFFENBACH/TALHAUSER, Was die Pestilentz an jr selbs sey [Augsburg: Philipp Ulhart $1535]$.

${ }^{521}$ StAA, CM IV, Nr. 13: Pestverordnungen des Rates (1535).

${ }^{522}$ Bernhard Schludin stammte aus Lindau und war Sohn eines Kapitäns. Wie aus seinem Schreiben an den Rat hervorgeht, zog er um 1530 nach Augsburg. Kurze Notiz zu seinem Leben in: MARTZ, Gesundheitswesen, S. 27.

${ }^{523}$ StAA, CM IV, Nr. 24: Supplikation von Doktor Schludin an den Rat (aus dem Inhalt für 1535 datierbar); StAA, Schätze, Nr. 16, p. 8R-11: Pestverordnungen des Rates pro Memoria (1535).
} 
Schludin berichtete davon, dass er sich vor einigen Jahren mit der Erlaubnis des Rates in Augsburg niedergelassen habe, um hier seine Tätigkeit als Leib- und Wundarzt auszuüben. Da er aber nachher mehr arme als reiche Leute, meistens für einen bescheidenen Lohn oder sogar umsonst kurieren müsste, geriet er bald in finanzielle Nöte. Aus diesem Grund wandte er sich an den Rat mit der Bitte um eine geringe städtische Besoldung, von der er nun leben könne, bis eine von den vier Stellen der eingeschworenen Doktoren wieder zu besetzten sei. Als Gegenleistung dafür wolle er seine medizinischen Fähigkeiten zum Wohl der ganzen Stadtgemeinde anbieten, die insbesondere in der gegenwärtigen wie auch in der künftigen Pestzeit erforderlich wären. Nach seiner Ansicht könnten vier Stadtärzte während der Epidemie für die Versorgung einer so zahlreichen Bevölkerung wie in Augsburg sicher nicht ausreichen. Da er aber wusste, dass die Ratsherren zurzeit keinen fünften Doktor in den städtischen Dienst aufnehmen wollten, ersuchte er sie nur um eine befristete Arztstelle, die der pestilentz zugehörig miest sein. Doktor Schludin bemerkte in diesem Zusammenhang, dass er sein Bittgesuch aus prophylaktischen Gründen schriftlich vorlegen müsse. Sein persönliches Erscheinen hätte nämlich von den Ratsherren missbilligt werden können, weil er gegenwärtig auch mit der Pest befallene Menschen besuche. Die Supplikation wurde vom Rat positiv beschieden, worauf die anschließende Anstellung von Doktor Schludin auf ein Jahr mit der Besoldung von 32 Gulden erfolgte.

Abgesehen von dieser speziellen Stelle für einen gelehrten Arzt lässt sich auch ein weiterer Ausbau des städtischen Personals in den Brechhäusern beobachten ${ }^{524}$. Zur Krankenbehandlung war dort Meister Heinrich, ein Barbier ernannt, der für seinen Dienst wöchentlich zwei Gulden und pro Quartal noch zwei weitere Gulden für das Bad erhielt. Sowohl der Doktor wie auch der Barbier hatten zu ihrer Hilfe je einen Knecht, der mit einem halben pro Woche Gulden besoldet war. Der Knecht des Doktors musste vor allem die verordneten Arzneimittel aus der Apotheke abholen, der andere Knecht war dem Barbier bei der Ausführung seiner wundarzneilichen Behandlung behilflich. Die alltägliche Verwaltung der Hospitaleinrichtung leiteten nach wie vor der Brechvater und die Brechmutter, die dafür einen Gulden pro Woche erhielten. Nach den Anweisungen des Rates sollten sie sich möglichst wenig unter den Kranken aufhalten und aus prophylaktischen Gründen keinen Kontakt zu den übrigen Stadtbewohnern pflegen.

Anstatt des Priesters wurde dagegen ein spezieller Vorsteher (furstentter) in den Brechhäusern mit einem Gulden wöchentlich besoldet, der die dortigen Kranken mit der religiösen Unterweisung und Trostspendung versehen sollte. Der Verzicht auf die Anstellung eines

\footnotetext{
${ }^{524}$ StAA, CM IV, Nr. 13: Begräbnis- und Pestverordnungen des Rates (1535).
} 
Priesters wie noch im Jahr 1521 bezeugt die wachsende Einflussnahme der Reformation in Augsburg um diese Zeit, wodurch die katholische Kirche auch aus diesem Bereich verdrängt wurde.

Darüber hinaus verordnete man einen Aufseher (sperrer) zur Aufbewahrung und Verteilung der Nahrungsmittel, eine Köchin und eine Wäscherin, die zu den wichtigsten Dienstleuten gehörten und wöchentlich einen halben Gulden Lohn erhielten. Daneben waren bis zu zehn Mägden und Knechte in den Brechhäusern tätig, die sich laut den Verordnungen des Rates mit den alltäglichen Hospitalarbeiten wie der Aufwartung der Kranken, Aufräumarbeiten, Nachtwachen oder dem Einnähen der Toten in die Leichensäcke beschäftigen sollten. Jede dieser Personen wurde wöchentlich mit 20 Kreuzern entlohnt. Für dieselbe Besoldung wurde auch ein Totengräber angestellt, der auf dem benachbarten Friedhof die Toten aus den Brechhäusern begraben sollte. Sämtliche Kleidung der verstorbenen Insassen musste er zur Lagerung unter der Aufsicht des Brechvaters übergeben, die auf den Befehl der Pfleger nach dem Ablauf der Epidemie ausnahmslos verbrannt wurde. Zur Kontrolle des Tores auf dem Hospitalgelände war vorübergehend auch ein Knecht mit der wöchentlichen Besoldung von 8 Kreuzern angestellt und dann aber entlassen worden, weil er laut der kurzen Bemerkung in der Pestverordnung sich übel verhielt oder nicht mehr notwendig sei.

Die Versorgung mit Lebensmitteln fand in enger Kooperation mit dem städtischen Blatterhaus statt, dessen Pfleger sowie der Blatterhausvater dafür direkt verantwortlich waren. Davon war einzig die Übergabe des Weins ausgenommen, die von den Baumeistern in die Brechhäuser verordnet und abgerechnet wurde ${ }^{525}$. Die Lieferungen aus dem Blatterhaus gingen dabei nach einem bestimmten Plan vor sich, wodurch man einer weiteren Infektionsverbreitung möglichst vorbeugen wollte. Ein Knecht aus der Stadt fuhr mit einer beladenen Karre bis vor die Brechhäuser und signalisierte seine Ankunft mit Blasen eines Hornes, damit die dortigen Dienstleute herauskamen und die gelieferten Nahrungsmittel abholten. Das Blatterhaus erfüllte auch eine Vermittlungsfunktion zwischen der Stadtgemeinde und den in den Brechhäusern angestellten Personen. Weil diese ihren Dienstort wegen der Ansteckungsgefahr nicht verlassen durften, half ihnen das Blatterhaus bei der Erledigung dringender Angelegenheiten in der Stadt. Der Brechvater bereite dazu jeden Samstag einen Zettel mit den Angaben vor, was für seine Dienstleute in Augsburg zu beschaffen wäre. Daneben wurde täglich eine Berechnung für das Blatterhaus zusammengestellt, in der notiert

\footnotetext{
525 Die wichtigsten Angestellten in den Pesthäusern erhielten 2 Maß Wein pro Tag, wobei den anderen Dienstleuten nur $1 \mathrm{Maß}$ ausgeschenkt wurde, in: StAA, CM IV, Nr. 13: Begräbnis- und Pestverordnungen des Rates (1535).
} 
wurde, welche Anzahl von Kranken dort aufgenommen wurde und wie viele Insassen inzwischen starben.

Die Brechhäuser waren also institutionell mit dem Blatterhaus verbunden, dessen Pfleger als die direkten Verwalter dieser beiden städtischen Hospitaleinrichtungen fungierten. Außer den Lebensmitteln besorgte das Blatterhausamt auch Bettzeug und Kleidung für die Insassen der Brechhäuser, die vornehmlich von den Altkleiderhändlern angekauft wurden. Ein derartiger Handel war in der Pestzeit generell verboten, so dass man nur ausnahmsweise - mit der Erlaubnis des Rates - solche Ankäufe in der Stadt durchführen konnte. Derartige Sachen durften jedoch keinesfalls aus den Häusern angekauft werden, in denen die Bewohner an der Pest erkrankt waren. Darüber hinaus waren die Pfleger des Blatterhauses offensichtlich auch für die Ausführung der anderen obrigkeitlichen Maßnahmen in der Pestzeit zuständig. Nach den Verordnungen des Rates sollten sie nämlich die Tätigkeit der Sesselträger und der städtischen Dienstleute auf den Gottesackern beaufsichtigen. Wenn die Pfleger selbst die diesbezüglichen Angelegenheiten nicht regeln konnten, sollten sie darüber direkt den Rat oder die Bürgermeister benachrichtigen. Das Blatterhausamt war also offenbar die wichtigste Institution in Augsburg, die mit der direkten Umsetzung der städtischen Pestbewältigung beauftragt wurde.

\section{Die Pestepidemie während des Augsburger Reichstages in den Jahren 1547-1548}

Die bis zur Mitte des 16. Jahrhunderts letzte Pestepidemie brach im August 1547 aus und dauerte bis zum Februar 1548 an $^{526}$. Die Sterblichkeitsrate war in dieser Zeit deutlich höher als während der vorherigen Seuchen. Nach den Angaben der Bevölkerungstafel starben in den Jahren 1547 und 1548 insgesamt 3.480 sowie 1.227 Menschen in Augsburg ${ }^{527}$. Der wichtigste Grund dafür war mutmaßlich die erhöhte Ansteckungsgefahr aufgrund der großen in der Stadt versammelten Bevölkerungsmenge. Die vielen Menschen waren anlässlich des vom 1. September 1547 bis zum 30. Juni 1548 unter Kaiser Karl V. abgehaltenen Reichstages nach Augsburg gekommen ${ }^{528}$. Angesichts der um sich greifenden Pest erarbeitete der Rat ab dem

\footnotetext{
${ }^{526}$ StAA, Ratsprotokolle, 1547: Fol. 13; 1548: Fol. 46v. Der Ausbruch dieser Pestepidemie wurde auch in den Chroniken kurz verzeichnet: SStBA, $2^{\circ}$ Cod. Aug. 53, Fol. 167v; GaSSER, Chronik, T. 3, S. 65; SteTten, Geschichte, VIII. 31. 417, 32, 429.

${ }^{527}$ Im Jahr 1546 und 1549 betrugen die jährlichen Mortalitätsraten in Augsburg 1.356 bzw. 1.757 Toten, in: Augsburger Bevölkerungstafel, SStBA, 2 Lw Einblattdrucke nach 1500, Nr. 347 (1511-1584).

${ }^{528}$ Zum Verlauf des Reichstages 1547/48 in Augsburg in: MACHOCZEK, Der Reichstag zu Augsburg 1547/48, S. 60-105.
} 
27. August 1547 die zu treffenden Vorsorgemaßnahmen und stimmte sich dabei auch mit den kaiserlichen Beratern $\mathrm{ab}^{529}$. Weil die Ratsherren während des Reichstags mit der Vielzahl der Angelegenheiten überfordert waren, ernannten sie am 22. September spezielle Pfleger für die Brechhäuser, die ab diesem Zeitpunkt direkt für die Organisation der städtischen Pestbewältigung zuständig waren ${ }^{530}$. Diese Bevollmächtigten des Rates arbeiteten an einem Entwurf der städtischen Pestordnung, die bereits am 29. September vollendet war und anschließend als kaiserlicher Befehl erlassen wurde ${ }^{531}$. Die darin festgelegten Anordnungen wurden nach demselben Muster wie 1535 formuliert, jedoch in einigen Punkten wegen des stattfindenden Reichstages beträchtlich verändert. Schon in der Einleitung dieser kaiserlichen Pestordnung erläuterte man den Ursprung der gegenwärtigen Epidemie auf andere Art und Weise. In dieser Hinsicht vermerkte man, dass sich wegen der großen in Augsburg befindlichen Volksmenge und aufgrund anderer Ursachen sich leichtlich ain vergiftung des lufts und sterbsucht an dem gebrechen und andern kranckhaiten bey diser stat Augspurg zuotragen moechte. Um dieser Gefahr wirksam entgegenzutreten zu können, befahl der Kaiser spezielle Verordnungen zu erlassen, die sowohl die Augsburger wie auch alle Ausländer, was stands, grads, wesens oder nation die seyen bei der Androhung von ernstlichen Strafen zu beachten hätten. Obwohl man die religiöse Deutung der Pest in diesen einleitenden Bemerkungen nicht anführte, war diese zeitgenössische Vorstellung nach wie vor präsent und zwar indem man zunächst eine Aufforderung zur frommen Hingabe an Gott verzeichnete. Der grundsätzliche Unterschied zum Ratserlass von 1535 bestand aber darin, dass die Maßregeln beträchtlich verschärft wurden. Diese Tendenz ist u.a. deutlich in den aufzuerlegenden Geldstrafen $\mathrm{zu}$ erkennen, die im Allgemeinen verdoppelt wurden. Die wichtigsten Veränderungen betrafen jedoch die Quarantäne der Pestkranken und ihrer Mitbewohnern, die nun restriktiv durchgeführt werden sollte. Alle Stadtbewohner und alle Fremden, die an der Pest erkrankten, mussten dem kaiserlichen Befehl nach mitsamt ihrer Kleidung in die städtischen Brechhäusern abgesondert und dort versorgt werden. Laut den Ratsprotokollen verordnete man zur Unterbringung der Pestkranken zusätzlich die Hütten am Lech, in die

\footnotetext{
${ }^{529}$ StAA, Ratsprotokolle, 1547: Fol. 13, 26, 27v, 28v, 29; 1548: Fol. 46v.

${ }^{530}$ Für die Wahrnehmung dieser Verwaltungsaufgaben wurden vermutlich die Ratsherren Bernhart Walther, Hanns Schaller und Heinrich Haisermann ernannt. Sie wurden nämlich am 29. September 1547 zur direkten Umsetzung der festgelegten Anweisungen verordnet.

${ }^{531}$ Der Text der Augsburger Pestordnung abgedruckt in: MACHOCZEK, Der Reichstag zu Augsburg 1547/48, S. 206-210: Ordnung zu Augspurg im reichstag anno 1548 contra morbum pestilentialem per romanorem cesarem uffgericht anno etc. 1548. Trotz der Datierung dieser Pestordnung auf das Jahr 1548 kann man ihre erste Verlautbarung in der Stadt schon für den Herbst 1547 voraussetzten. Darauf weisen die Aufzeichnungen in den Ratsprotokollen hin. Der handschriftliche Entwurf dieses Textes wurde zudem am Rande mit der Jahresangabe 1547 markiert, in: StAA, CM 4, Nr. 13: Die Pestordnung (1547).
} 
vornehmlich die Fremden eingewiesen werden sollten ${ }^{532}$. Die Mitbewohner der infizierten Personen mussten nun 14 Tage in ihren Häusern ausharren oder die Stadt sofort verlassen. Die städtischen Bediensteten sollten den eingesperrten Hausbewohnern alles Notwendige liefern. Die Vermögenden sollten dafür eine kleine Gebühr entrichten und die Armen vom Almosenamt unterstützt werden. Die dazu verordneten Dienstleute mussten sich beständig an vier Punkten in der Stadt aufhalten, um von den Bedürftigen leicht benachrichtigt zu werden. Auch die Begräbnisse sollten jetzt nur noch an zwei Tageszeiten stattfinden: morgens von 5 bis 7 Uhr und abends von 18 bis 19 Uhr, damit man nicht am hellen Tage die Toten zu Grabe trüge. In der kaiserlichen Pestordnung wurde dagegen die Bestimmung von 1535 über die Organisation des Begräbniswesens auf nur zwei Gottesäckern nicht verlautbart. Daraus kann jedoch nicht geschlussfolgert werden, dass mit der Rekatholisierung Augsburgs unter dem kaiserlichen Schutz diese Anordnung wegen ihrer möglichen Konnotation mit dem Luthertum aufgegeben wurde ${ }^{533}$. Ein Eintrag in den Ratsprotokollen weist nämlich daraufhin, dass die innerstädtischen Begräbnisse während der Pestzeit nach wie vor von der städtischen Obrigkeit untersagt worden seien. Am 1. März 1548 erlaubte der Rat eine verstorbene Stadtbewohnerin namens Arnoldin in ihrem Grab bei der St. Moritz Kirche zu bestatten, mit der Begründung, dass sich die Epidemie in Augsburg völlig nachgelassen habe und seit drei Wochen kein Mensch mehr an der Pest gestorben sei $^{534}$. Diese medizinische Schutzmaßnahme wurde also auch nach der Restitution des Katholizismus in Augsburg eingehalten. In diesem Kontext kann man daher einen fundamentalen Wandel im Umgang mit den Toten erkennen, der sich nach der Durchsetzung des medizinischen Diskurses ungeachtet der konfessionellen Unterschiede vollzog. Diese kulturelle Umwandlung wurde von Craig Koslofsky als „die Transformation der traditionellen christlichen Gemeinschaft von Lebenden und Toten zu einem durch Hygiene bestimmten Gemeinwesen der Lebenden“ charakterisiert, die sich im Fall Augsburgs schon seit dem Ende des 15. Jahrhunderts schrittweise durchzusetzen begann $^{535}$.

Als sich die Epidemie an vielen Orten in der Stadt weiter ausbreitete, erließ man am 18. Oktober 1547 eine Anordnung zum vermehrten Schutz des Rates vor der Infektionsgefahr ${ }^{536}$. Die Parteien, die vor den Ratsherren ihre Angelegenheiten anbringen

\footnotetext{
${ }^{532}$ StAA, Ratsprotokolle, 1547: Fol. 28v.

${ }^{533}$ Siehe S. 112, 154-156.

${ }^{534}$ StAA, Ratsprotokolle, 1547: Fol. 46v.

${ }^{535}$ KOSLOFSKY, >Pest $<$ - >Gift $<$ - >Ketzerei<, S. 207. In Leipzig, wo 1536 eine konfessionelle Kontroverse um eine neue Begräbnisordnung ausbrach, wurde das Verbot der innerstädtischen Bestattungen vom katholischen Herzog mit Ausnahme für die dagegen protestierenden Professoren, Magister und Studenten der dortigen Universität eingeführt.

${ }^{536}$ StAA, Ratsprotokolle, 1547: Fol. 39.
} 
wollten, durften seither nicht mehr persönlich erscheinen, sondern mussten ihre Supplikationen schriftlich vorlegen und geduldig eine Antwort der entsprechenden Stadtbehörden abwarten. Auch die Personen, die mit der Verwaltung von städtischen Pflegschaften und ähnlichen Ämtern beauftragt waren, sollten nur noch von einigen Ratsmitgliedern empfangen und nicht mehr zum gesamten Rat vorgelassen werden. Man erließ auch eine Verordnung zur Ausführung besonderer Reinigungsmaßnahmen im städtischen Raum ${ }^{537}$. Nach dem Befehl des Rates sollten sich alle Leute um die Sauberkeit in ihren Häusern, auf den Straßen und Plätzen sowie insbesondere in den Röhrkästen und bei den Metzgern bemühen. Demgemäß mussten die Stadtbewohner innerhalb von fünf Tagen nach der obrigkeitlichen Verordnung Kehricht, Kot, Mist, Lumpen oder anderen Müll aus der Stadt wegbringen. Der Rat begründete diese Maßnahme damit, dass die Unsauberkeit nicht nur den guten Ruf der Stadt beeinträchtigte, sondern vor allem zur Vergiftung der Luft beitrüge, was die Verbreitung von Krankheiten unter so zahlreich anwesenden Menschen weiter begünstige. Daher solle jeder Stadtbewohner, vor dessen Haus solche Abfälle aufgefunden würden, vom Rat ernsthaft bestraft werden.

\section{Anordnungen zum Bettelwesen im Kontext der Seuchenbewältigung in der ersten Hälfte des 16. Jahrhunderts}

Unter den kaiserlichen Anordnungen für die Organisation des Reichstages findet sich auch ein Erlass gegen herumziehende Bettler, der in direkten Zusammenhang mit der Gefahr der weiteren Verbreitung der Seuche gebracht wurde ${ }^{538}$. Nach damaliger Erkenntnis konnten nämlich infolge des Zusammentreffens großer Menschenmengen pestilentzen und andere beschwaerliche kranckhaiten ausbrechen. Die Seuchen entsprängen insbesondere dadurch, wenn die Bettler mit iren kranckhaiten und erbseüchten in die Städte eingelassen würden. Aus diesem Grund wurde auf Befehl des Kaisers angeordnet, dass alle Bettler Augsburg sofort verlassen müssten. Der Rat solle dabei alle zur Verfügung stehenden Möglichkeiten in Anspruch nehmen, damit diese Verordnung wirklich ausgeführt und eingehalten werden könnte. Ein ähnliches Einlassverbot für Bettler hatte der Rat - wegen bestehender Seuchengefahr - bereits 1521 beschlossen ${ }^{539}$.

\footnotetext{
${ }^{537}$ StAA, Schätze, Nr. 16, p. 128R-129R: Ratsdekret wegen des Reichtags (22. November 1547).

${ }^{538}$ MACHOCZEK, Der Reichstag zu Augsburg 1547/48, S. 204-206: Ordnung, wie es mit frembden und anderen stenden uff dem reichstag zu Augspurg gehalten werden solle, anno etc. 1548. Hier S. 206.

${ }^{539}$ Siehe S. 135.
} 
Im Zusammenhang mit diesen Anordnungen kann man auch annehmen, dass die Einführung des generellen Bettelverbots von 1541 in Augsburg auch von der Entwicklung der städtischen Pestpolitik beeinflusst wurde ${ }^{540}$. Die ersten Regelungen zur Einschränkung des Bettelwesens sind schon ab Mitte des 15. Jahrhunderts zu beobachten, als zu diesem Zweck die ersten Bettelordnungen vom Rat erlassen wurden ${ }^{541}$. Dabei konnte die Bettelei erst mit der Gründung des Almosenamtes, das bemerkenswerterweise sofort nach dem Ende der Pestepidemie im Jahre 1522 entstand, unter strengere Kontrolle der Stadtbehörden gestellt werden. Der Rat ernannte damals sechs Almosenherren, die mit Hilfe der dazu verordneten Knechte an Sonn- und Feiertagen die Almosen in den Pfarreien und Klöstern oder auch von Haus zu Haus gehend sammelten, welche anschließend unter den Bedürftigen in der Stadt verteilt wurden. Die Armen selbst durften noch nur mit Erlaubnis der Almosenherren vor den Kirchen und an öffentlichen Orten betteln. Die weitere Reorganisation des Armenwesens erfolgte im Jahr 1541, als zwei wichtige Veränderungen eingeführt wurden: 1. das völlige Verbot des Bettelns und 2. die Verteilung der Almosen vor allem in Form von Naturalien ${ }^{542}$.

In der Forschungsliteratur werden die Augsburger Armenordnungen von 1522 und 1541 allgemein als Ausdruck obrigkeitlicher Zentralisierung der Armenfürsorge gedeutet, wodurch die Almosengaben unter städtischer Kontrolle zielgerecht an die Bedürftigen verteilt werden konnten. Dabei wurde in diesem Bereich auch die Einflussnahme der Reformation hervorgehoben, die vor allem mit dem generellen Verbot des Bettelns von 1541 in Augsburg deutlich zutage trat. Nach Luthers Lehre sollte nämlich jede christliche Gemeinde durch eine geregelte Armenfürsorge das Betteln der eigenen Bürger überflüssig machen. Die Auswirkungen der städtischen Pestpolitik in der ersten Hälfte des 16. Jahrhunderts auf die Regelungen des Bettelwesens sind dagegen in diesen Studien völlig unbeachtet geblieben. Die zeitgenössische Vorstellung, dass die Bettler für die weitere Verbreitung von Seuchen verantwortlich seien, konnte sicherlich - neben den in der Forschung immer wieder angeführten Faktoren - die städtische Organisation des Armenwesens beträchtlich prägen. Die wichtige Rolle dieser zeitgenössischen Wahrnehmung ist dabei schon in den dargestellten Verordnungen gegen die Bettler von 1520 und 1548 eindeutig zu erkennen. Das Verbot des

\footnotetext{
${ }^{540}$ Die Augsburger Armenordnung von 1541 abgedruckt in: ROTH, Reformationsgeschichte, Bd. III, S. 207.

541 Die einschlägigen Studien zur Armenfürsorge im frühneuzeitlichen Augsburg vor allem in: CLASEN, Armenfürsorge (1-2); BISLE, Armenpflege, S. 5ff. Zur Augsburger Armenordnung von 1521 in: JÜTTE, Armenfürsorge, S. 41; WincKELMANN, Armenordnungen, S. 212. Allgemein zur Sozialgeschichte der Armut in der Frühen Neuzeit: JÜTTE, Arme, Bettler, Beutelschneider.

${ }^{542}$ Mit der Armenordnung von 1541 konnte vor allem die Bettelei hiesiger Armer unter städtische Kontrolle gebracht und weitgehend abgeschafft werden, da der Zufluss von auswärtigen Bettlern nach Augsburg ein ständiges Problem für die städtische Obrigkeit in der Frühen Neuzeit bildete, dazu in: CLASEN, Armenfürsorge (2), S. 107-115.
} 
Bettelns im Jahr 1541 kann man daher im Kontext der städtischen Pestpolitik auch als eine weitere Schutzmaßnahme vor der Infektionsgefahr ansehen. 


\section{Die soziale Konstruktion der Pest in Augsburg um 1500 - Einblick in den historischen Hintergrund}

Wie in den vorhergehenden Kapiteln dargestellt wurde, entwickelte sich seit dem Ende des 15. Jahrhunderts die soziale Konstruktion der Pest in Augsburg, deren Grundlagen von einem engen Zusammenspiel des religiösen und medizinischen Diskurses geprägt waren. Die beiden diskursiven Ansätze entstanden dabei schon nach dem Ausbruch des „Schwarzen Todes“ in der Mitte des 14. Jahrhunderts, als die noch unbekannte Seuche sowohl von Geistlichen als auch von gelehrten Medizinern in Europa nach den zeitgenössischen Wahrnehmungsmustern expliziert wurde. Diese zeitgenössischen Deutungen wurden jedoch keinesfalls gleichzeitig in Augsburg rezipiert. Die Auslegung der Pest als Strafe Gottes war nämlich schon während der ersten Epidemien allgemein präsent, was sich in der Ausübung von Prozessionen, Zelebrierung von Pestmessen oder Bittgebeten an die Pestheiligen anschaulich widerspiegelte. Die religiöse Auffassung der Seuche wurde dabei vor allem von der Kirche unter der Stadtbevölkerung verbreitet und bildete seither einen festen Bestandteil der sozialen Konstruktion der Pest. Die Pestlehre der Mediziner, die nach den galenisch-hippokratischen Grundsätzen ebenfalls in der Mitte des 14. Jahrhunderts in zahlreichen Traktaten formuliert wurde, besaß dagegen um diese Zeit noch keine größere Relevanz in Augsburg. Erst seit dem Ende des 15. Jahrhunderts spielten die Lehren der zeitgenössischen Medizin eine immer wichtigere Rolle, da die städtische Obrigkeit begann ihre Pestbewältigung in der Stadt aufgrund der Anweisungen von Medizinern zu organisieren. Dadurch vollzog sich eine fundamentale Umwandlung in der sozialen Konstruktion der Pest, die nun auch grundlegend vom medizinischen Diskurs bestimmt wurde. Diese Wende in Augsburg um 1500 ergibt sich aus der historischen Verflechtung verschiedener Faktoren.

\section{Aufstieg der gelehrten Mediziner zu städtischen Experten}

Die Voraussetzung für den kulturellen Wandel im Umgang mit der Pest war die Entwicklung einer neuen Wissensordnung, die sich mit der schrittweisen Verbreitung und Rezeption der medizinischen Vorstellungen etablieren konnte. Dieser Prozess setzte in Augsburg offensichtlich mit der Popularisierung der Pestlehre mittels des Buchdruckes ein, als seit den Siebziger Jahren des 15. Jahrhunderts die an ein breites Lesepublikum gerichteten 
Pestschriften vermehrt gedruckt werden konnten ${ }^{543}$. Dadurch entstand ein allgemeiner Hintergrund zur Durchsetzung des medizinischen Diskurses in breiteren Bevölkerungsschichten. Entscheidend war jedoch in diesem Prozess ein anderer Faktor: der Aufstieg gelehrter Mediziner zu städtischen Experten, von denen die gesundheitspolitischen Entscheidungen des Rates maßgebend mitgestaltet wurden. In der Forschung wird dabei die Bedeutung der akademisch gebildeten Ärzte in diesem Zusammenhang umstritten bewertet. Vor allem in den neueren Arbeiten ist immer wieder die Ansicht vertreten, dass die obrigkeitlichen Maßnahmen zur Pestbewältigung nicht direkt aufgrund der medizinischen Lehre ergriffen wurden, sondern sich eher auf den allgemeinen Beobachtungen der Machtinhaber über Ansteckungsverläufe in der Pestzeit stützten ${ }^{544}$. Demzufolge spielten die Doktoren keine wichtige Rolle bei der Anordnung der obrigkeitlichen Pestpolitik und besaßen lediglich eine ausführende Funktion. Im Gegensatz zu solchen Annahmen lässt sich jedoch für Augsburg eindeutig aufzeigen, dass die Stadtärzte an der Regelung des städtischen Medizinalwesens auf entscheidende Weise beteiligt waren.

Die in der Reichsstadt praktizierenden Ärzte lassen sich erst in der zweiten Hälfte des 15. Jahrhunderts als zahlenmäßig relevante Gruppe in den Quellen fassen; ihre Gesamtzahl konnte bis zu 6 Personen betragen ${ }^{545}$. Davon waren zumeist zwei Doktoren als Stadtärzte vom Rat angestellt. Die Zahl der studierten Mediziner in Augsburg stieg weiterhin an, so dass in der ersten Hälfte des 16. Jahrhunderts zwischen 4 und 11 Doktoren in der Reichstadt tätig waren, wobei sich die Gesamtzahl der von der Stadt besoldeten Ärzte auf vier erhöhte ${ }^{546}$. Die beträchtliche Zunahme der gelehrten Ärzteschaft in Augsburg im gesamten 16. Jahrhundert verdeutlicht die von Werner Dieminger zusammengestellte Tabelle:

\footnotetext{
${ }^{543}$ Siehe Kapitel III, S. 44f.

${ }^{544}$ Die kritische Einschätzung der Rolle von gelehrten Medizinern bei der obrigkeitlichen Pestbewältigung ist vor allem in den folgenden Arbeiten anzutreffen: CARMiCHAEL, Plague and the poor, S. 112; DingES, Pest und Pestpolitik, S. 295; DERS., Pest und Staat, S. 75; KInZELBACH, Gesundbleiben, S. 243-245. Dagegen wurden die Ärzte als wichtiges Element der organisierten Pestbekämpfung dargelegt in: CIPOLLA, Public Health, S. 7, 11-17, 20-22; FISCHER, Gesundheitswesen, Bd. I, S. 162; HENDERSON, Epidemics, S. 165-186.

545 Vor der Mitte des 15. Jahrhundert lassen sich nur einzelne Mediziner in Augsburg belegen, die ihre medizinische Ausbildung vermutlich an den Universitäten erwarben. Ein vermehrtes Aufkommen von Doktoren ist jedoch erst um 1450 nachweisbar, dazu siehe S. 40-41.

${ }^{546}$ DIEMINGER, Bader, S. 32.
} 
Tab. 1: Entwicklung der Medizinerzahl im 16. Jahrhundert in Augsburg (Dieminger 1999, S. 32)

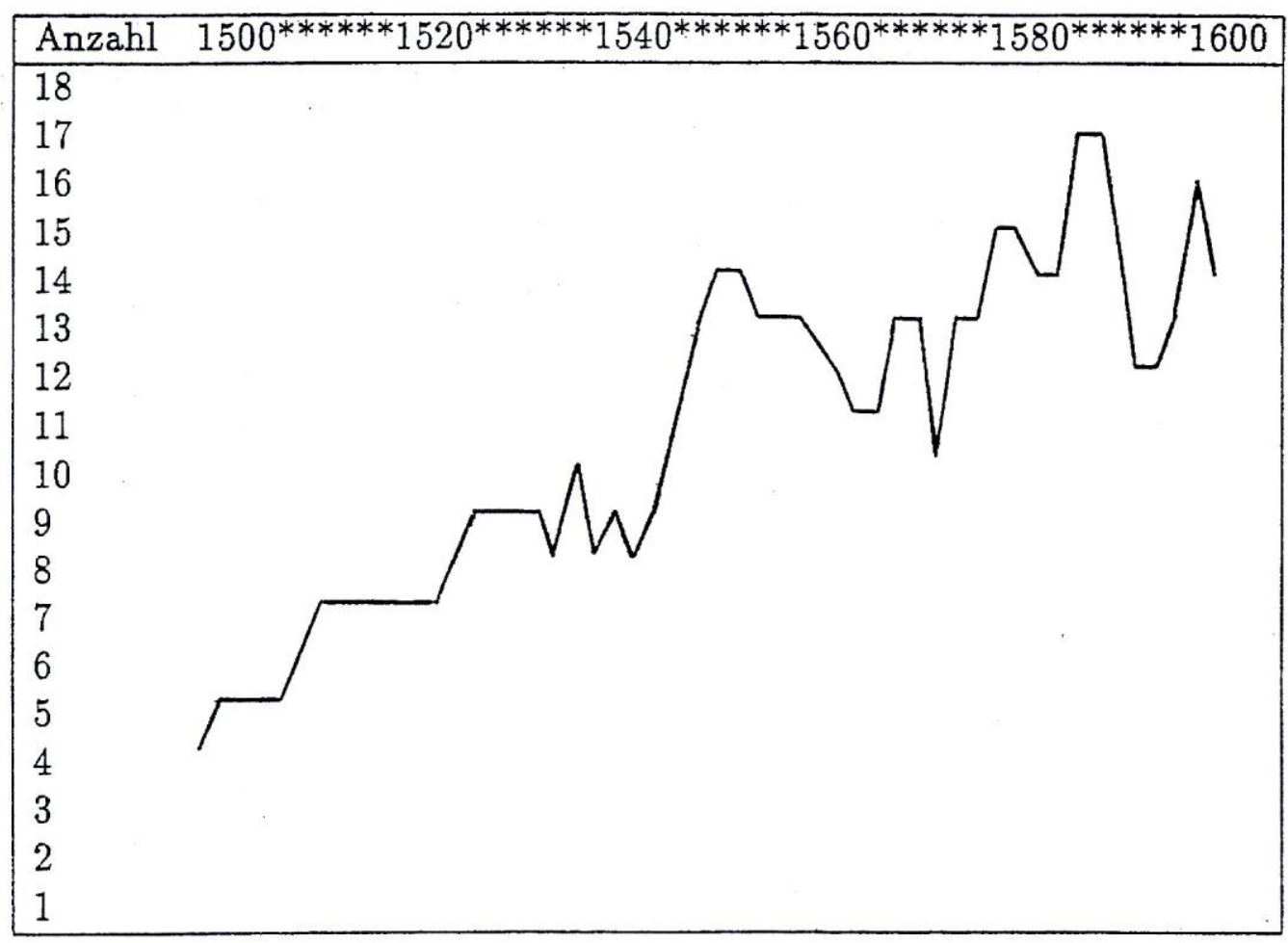

Die weitgehenden Veränderungen auf dem Augsburger Medizinermarkt bestätigen auch Diemingers andere statistische Erhebungen ${ }^{547}$. Die Versorgung der Augsburger Bevölkerung mit gelehrten Ärzten verbesserte sich ab Anfang des 16. Jahrhunderts weiter, so dass sich ihre Anzahl im Verhältnis zur Bevölkerung bis 1550 verdoppelte:

Tab. 2: Versorgung der Augsburger Bevölkerung mit studierten Ärzten im 16. und beginnenden 17. Jahrhundert (Dieminger 1999, S. 33)

\begin{tabular}{|l|l|l|l|l|l|l|l|}
\hline Jahr & 1500 & 1510 & 1520 & 1530 & 1540 & 1550 & 1560 \\
\hline Einw./Med. & 7500 & 6158 & 4511 & 3596 & 3316 & 3086 & 2672 \\
\hline Jahr & 1570 & 1580 & 1590 & 1600 & 1610 & 1615 & 1619 \\
\hline Einw./Med. & 3230 & 2793 & 2319 & 2915 & 2418 & 2057 & 1576 \\
\hline
\end{tabular}

\footnotetext{
${ }^{547}$ DERS., S. 33, 37.
} 
Parallel dazu verschlechterte sich in dieser Zeit die gesamte Versorgung mit handwerklichen Medizinern: Bader, Barbiere und Wundärzte. Der wichtigste Grund dafür scheint vor allem in ihrer konstanten Gesamtzahl im Verhältnis zum raschen Zuwachs der Gesamtbevölkerung in Augsburg zu liegen. Auch die größere Mortälitätsrate innerhalb dieser Medizinergruppe, die sich während der Pestepidemien mit der wundarzneilichen Behandlung der Beulen befasste und dadurch anscheinend häufiger als die gelehrten Ärzte in direktem Kontakt mit den Kranken stand, könnte dafür verantwortlich sein:

Tab. 3: Versorgung der Augsburger Bevölkerung mit Handwerkschirurgen im 16. und beginnenden 17. Jahrhundert (Dieminger 1999, S. 37)

\begin{tabular}{|l|l|l|l|l|l|l|l|l|}
\hline Jahr & 1501 & 1516 & 1550 & 1568 & 1583 & 1610 & 1615 & 1619 \\
\hline Einw./Hch. & 911 & 1052 & 1371 & 1339 & 2215 & 624 & 542 & 588 \\
\hline
\end{tabular}

Aus diesen Gründen nahm die Gesamtmenge von Medizinern in Augsburg bis zum Ende des 16. Jahrhunderts beträchtlich ab:

Tab. 4: Die medizinische Versorgung der Augsburger Bevölkerung mit Heilkundigen im 16. und beginnenden 17. Jahrhundert (Dieminger 1999, S. 37)

\begin{tabular}{|l|l|l|l|l|l|l|l|l|}
\hline Jahr & 1501 & 1516 & 1550 & 1568 & 1583 & 1610 & 1615 & 1619 \\
\hline Einw./Heilk. & 813 & 852 & 950 & 945 & 1161 & 496 & 430 & 428 \\
\hline
\end{tabular}

Das zahlenmäßige Verhältnis der Doktoren zu den handwerklich ausgebildeten Medizinern verschiebt sich also im 16. Jahrhundert zunehmend zu Gunsten der ersten Gruppe. 1501 kamen statistisch auf einen gelehrten Arzt fünf Bader, 1550 etwa zwei Doktoren auf fünf Bader, während ihr Verhältnis im Jahre 1611 sogar eins zu drei ausmachte.

Außer der quantitativen Steigerung der gelehrten Ärzteschaft ist auch der Verlauf ihrer Universitätsausbildung sehr aufschlussreich. Die Ärzte in Augsburg reisten bis zur Mitte des 16. Jahrhunderts vornehmlich nach Italien, um dort ihr Doktorstudium zu absolvieren ${ }^{548}$.

\footnotetext{
${ }^{548}$ MARTZ, Gesundheitswesen, S. 66. In der zweiten Hälfte des 16. Jahrhunderts wurden die Augsburger Doktoren dagegen vorwiegend an deutschsprachigen Universitäten promoviert, darunter in erster Linie an der Universität in Basel. Die Aufzählung der nachweisbaren Studienorte von gelehrten Augsburger Ärzten im gesamten 16. Jahrhundert ergibt folgendes Bild: der deutschsprachige Raum: Basel 15, Freiburg 2, Ingolstadt 4, Tübingen 6, Wien 1, Wittenberg 2; Frankreich: Avignon 1, Dole 1, Montpellier 5, Orange 1; Italien: Bologna 6, Ferrara 2, Florenz 2, Padua 13, Pisa 3, Siena 1; Spanien: Valencia 1.
} 
Von den 25 bis zum Jahre 1550 nachweisbaren Doktoren aus Augsburg wurden insgesamt 12 in den italienischen Städten Bologna, Ferrara, Padua, Perugia und Siena, sowie je einer in Ingolstadt und Avignon promoviert. Für die übrigen 11 Mediziner lassen sich solche Angaben nicht ermitteln. Eine derartige Auswahl von Studienorten unter den Augsburger Medizinern ist in diesem Kontext von größter Bedeutung, da die norditalienischen Städte als Vorreiter der institutionellen Pestbewältigung in Europa galten ${ }^{549}$. Schon im 15. Jahrhundert wurden in vielen norditalienischen Städten Vorsorge- und Bekämpfungsmaßnahmen von den Obrigkeiten verordnet, denen jeweils die Erkenntnisse der damaligen medizinischen Pestlehre zugrunde lagen. In Venedig gründete man auf den anliegenden Inseln in der Lagune das erste dauerhaft existierende Pesthospital in Europa: 1423 das Lazaretto Vecchio für die Kranken und im Jahr 1468 das Lazaretto Nuovo als Quarantänestation für Pestverdächtige aus der Stadt und für ankommende Kaufleute und Waren aus infizierten Gebieten. In Mailand wurde dagegen zwischen 1468 und 1488 ein geräumiges Pesthospital eingerichtet, das in vier spezielle Abteilungen für Kranke, Krankheitsverdächtige, Rekonvaleszente und Krankenpersonal untergliedert war. Diese Hospitaleinrichtungen sowie andere obrigkeitliche Pestverordnungen in Venedig und Mailand waren ein Vorbild für die Organisation der entsprechenden Maßnahmen in anderen norditalienischen Städten.

Im deutschsprachigen Raum setzte dagegen die medizinisch geprägte Pestpolitik der Städte grundsätzlich erst am Ende des 15 Jahrhunderts ein $^{550}$. Als ein allgemeiner Nachweis dafür können vor allem die ersten Quellenangaben über die Einrichtungen für die Pestkranken herangezogen werden. Abgesehen von einigen kleinen Häusern in Aachen (zweite Hälfte des 14. Jahrhunderts) und in Wien (erste Hälfte des 15. Jahrhunderts) sind die ersten Pesthäuser erst in der zweiten Hälfte des 15. Jahrhunderts in größerer Anzahl nachweisbar: Braunschweig (1473), Münster (1475), Augsburg (1494), Frankfurt/M. (1494), Celle (1495), Überlingen (1498) und Warendorf (Ende des 15. Jahrhunderts). Nach der Klassifikation von Otto Ulbricht sind dabei zwei Typen solcher Institutionen zu unterscheiden ${ }^{551}$ :

1. „Pesthäuser“: kleine Gebäude, meistens innerhalb der Stadt und in mitten anderer Häuser gelegen, die zur Unterbringung der Pestkranken während der Epidemien ausgestattet bzw. umgestaltet wurden;

\footnotetext{
549 PALMER, The Control, S. 183-194; DINGES, Süd-Nord-Gefälle; RoDENWALDT, Gesundheitsgesetzgebung; DERS., Pest in Venedig.

${ }^{550}$ UlBRICHT, Pesthospitäler, S. 96-103; DingES, Süd-Nord-Gefälle, S. 29-30.

${ }^{551}$ Diese Klassifikation basiert nicht auf den zeitgenössischen Quellenbezeichnungen, sondern berücksichtigt ausschließlich die jeweilige Organisation der Einrichtungen für die Pestkranken.
} 
2. „Pesthospitäler“: spezielle Gebäudekomplexe mit Friedhof und Kapelle für eine größere Anzahl von Kranken, die strikt nach den Grundsätzen der damaligen Pestlehre außerhalb der Stadtmauern erbaut wurden.

Demnach wurden die ersten „Pesthospitäler“ erst zu Beginn des 16. Jahrhunderts in den großen Städten im Süden des Reiches gegründet, von denen Nürnberg (1498 Baubeginn, 1520 erstmals genutzt), Frankfurt (1518), Ravensburg (Bauplan 1520) und Augsburg (1521) als Vorläufer gelten können. Dabei kann vorausgesetzt werden, dass die norditalienischen Lazarette ein wichtiges Vorbild für die Gründungen der ersten deutschen „Pesthospitäler“ darstellten, was vor allem durch die engen wirtschaftlichen Beziehungen zwischen diesen Gebieten begünstigt wurde. Auf einen diesbezüglichen Wissenstransfer weisen vor allem die Geschehnisse in Nürnberg direkt hin ${ }^{552}$. Im Jahre 1518 erwarb der dortige Rat die Hausordnungen von zwei Lazaretten in Venedig, auf deren Grundlage die Organisation des neu erbauten Sebastianhospitals in Nürnberg geregelt wurde. In diesem Zusammenhang ist davon auszugehen, dass die Studienaufenthalte der Mediziner an italienischen Universitäten zur Rezeption der dortigen gesundheitspolitischen Handlungsmuster in Augsburg beitrugen.

Für die erfolgreiche Durchsetzung von gelehrten Medizinern in Augsburg war der Umstand von Bedeutung, dass einige Doktoren schon seit dem Ende des 15. Jahrhunderts zu höchstem Ansehen in ihrer medizinischen Tätigkeit gelangten. Die berühmtesten Ärzte in der Stadt gehörten zur Familie Jung, die drei Generationen von prominenten Medizinern hervorbrachte ${ }^{553}$. Der aus Wimpfen am Neckar stammende Johann Jung (ca. 1435 - 1515) war zuerst als Stadtarzt in Ulm tätig und in den Jahren 1490-93 wurde er zum Leibarzt des Kaisers Maximilian I. ernannt. Im Anschluss daran zog er nach Augsburg, wo er als Stadtarzt mit 80 Gulden Jahressold angestellt wurde. Im Jahr 1494 erhielt er einen kaiserlichen Adelsbrief für sich und seine fünf Söhne, von denen Ambrosius (1471 - 1543) und Ulrich (1478-1539), wie bereits erwähnt, ebenfalls Ärzte wurden. Sie galten als die herausragendsten Mitglieder dieser Medizinerfamilie. Der Ältere, Ambrosius, war seit 1494 Leibarzt des Augsburger Domkapitels und in den Jahren 1510-1522 auch als Stadtarzt tätig. Seine eifrige Tätigkeit als Autor der in Augsburg beliebten Pestschriften wurde schon erläutert ${ }^{554}$. Ulrich Jung dagegen war seit 1507 Stadtarzt in Augsburg und im Jahr 1520 wurde er zum Leibarzt des Kaisers Karl V. und Königs Ferdinand I. ernannt. Im demselben Jahr erhielt er zusammen mit seinem Bruder Ambrosius einen erneuten kaiserlichen Adelsbrief. Am 11. Dezember

\footnotetext{
${ }^{552}$ ULBRICHT, Pesthospitäler, S. 104-105.

${ }^{553}$ FleischmanN, Ärztefamilie Jung; MARTZ, Gesundheitswesen, S. 18-20; GENSTHALER, Medizinalwesen, S. 43.

${ }^{554}$ Siehe S. 62-64.
} 
1538 wurden die beiden Brüder auch in den Patrizierstand in Augsburg aufgenommen. Ihr hohes Ansehen trat auch dadurch zutage, dass die wichtigsten Persönlichkeiten der Stadt, wie z.B. die Familie Fugger, ihre medizinische Kunst in Anspruch nahmen. Die medizinische Tradition der Familie Jung setzte dann Ambrosius II. (1510-1559) fort, der ein Sohn von Ambrosius Jung war. Auch er wurde 1544 als Stadtarzt in Augsburg angenommen, erreichte aber nicht die Bedeutung seines Vaters bzw. Onkels ${ }^{555}$.

Die zweite berühmte Ärztefamilie in Augsburg war die Familie Occo, aus der insgesamt vier Generationen Doktoren hervorgingen. Der Stammvater war Adolph Occo I. (1447-1503), der einem alten adligen Geschlecht aus Friesland entstammte ${ }^{556}$. Seit 1474 war er in Augsburg tätig, wo er insbesondere bei den Bischöfen Johann von Werdenberg und Friedrich von Hohenzollern ein großes Ansehen besaß. 1488 war er Hof- und Leibarzt bei Pfalzgraf Philipp in Heidelberg und in den Jahren 1490-1494 bei Erzherzog Sigmund von Österreich. Dieser auch als Humanist bekannte Arzt übte anschließend seine medizinische Tätigkeit bis zu seinem Tod in Augsburg aus, indem er vor allem die bedeutendsten Familien der Stadt kurierte. Sein Adoptivsohn Adolph Occo II. (1494-1572) wurde ebenfalls zum Doktor der Medizin ausgebildet und war seit 1523 lebenslang als angesehener Stadtarzt in Augsburg tätig. $\mathrm{Zu}$ besonderer Berühmtheit als Arzt gelangte auch sein Sohn Adolph Occo III. (15241606). Im Jahr 1564 verfasste er zusammen mit anderen Augsburger Doktoren das berühmte Medikamentenbuch „Pharmacopea Augustana“, das auch in anderen Städten weit verbreitet war und dort rezipiert wurde ${ }^{557}$. In Anerkennung seiner Tätigkeit verlieh ihm Kaiser Maximilian II. im Jahr 1573 eine goldene Verdienstmedaille und vermehrte sein Adelswappen mit einem sich in die Höhe schwingenden goldenen Adler im blauen Feld. Die ärztliche Tradition in der Familie Occo setzten auch zwei seiner Söhne fort: David (15671595) und Adolph IV. (1578-1628), von denen der Erste hauptsächlich in Wien und der Zweite lebenslang in Augsburg praktizierten.

Die Mediziner aus den Familien Jung und Occo erfreuten sich einer hohen sozialen Stellung, die außer den erwähnten Erhebungen in den Adel- oder Patrizierstand noch durch die Hochzeiten mit Töchtern aus vornehmen Familien gefestigt wurde. Dabei waren die Eheschließungen mit Frauen aus Adels-, Patrizier- und Kaufmannsfamilien auch für die anderen gelehrten Mediziner in Augsburg, insbesondere in der ersten Hälfte des 16.

\footnotetext{
555 Die Tochter von Ambrosius II. Jung, Regina heiratete dagegen den bekannten Arzt Leonhard Rauhwolff (1540-1596), der seit 1563 mit einigen Abständen auch als Stadtarzt in Augsburg tätig war.

${ }^{556}$ BÜHLER, Ärztegeschlecht der Occo; GenSTHALER, Medizinalwesen, S. 44-45; MARTZ, Gesundheitswesen, S. 21-24.

${ }^{557}$ Eine ausführliche Studie zur „Pharmacopea Augustana“ in: GENSTHALER, Medizinalwesen, S. 93-147.
} 
Jahrhunderts typisch ${ }^{558}$. Mit einer derartigen Heiratspolitik konnten die Ärzte nicht nur ihr Sozialprestige weiter erhöhen, sondern auch ihre persönlichen Kontakte zur gesellschaftlichen und politischen Führungsschicht in der Reichsstadt ausbauen. Daneben nahmen manche gelehrte Mediziner auch selbst an der Gestaltung des politischen und religiösen Lebens teil, indem sie sich vor allem während der Reformation aktiv in Augsburg engagierten ${ }^{559}$. Gereon Sailer (gest. 1562) war maßgeblich für die Berufung Straßburger Prediger nach Augsburg 1530/31 verantwortlich. 1535 wurde er vom Rat auch zu Martin Luther nach Wittenberg geschickt, um mit ihm über die Sakramentfrage zu verhandeln. Dagegen beriet Ambrosius Jung als weltlicher Kirchenpropst den Rat in kirchlichen Angelegenheiten. Die sozial hochgestellten Doktoren konnten daher auch dank ihrer engen verwandtschaftlichen Beziehungen zur sozialen Führungsschicht sowie durch ihr politisches Engagement die Augsburger Politik beeinflussen bzw. mitbestimmen. Ihr größtes Interesse richtete sich dabei auf das städtische Medizinalwesen, dessen Regelungen entscheidend von ihren Anweisungen geprägt wurden.

\section{Beratungen der Augsburger Stadtärzte zur Regelung des städtischen Medizinalwesens}

Die ersten gesundheitspolitischen Verordnungen des Rates kann man bei der Organisation des Apothekenwesens in Augsburg nachweisen ${ }^{560}$. Nachdem sich in der Mitte des 15. Jahrhunderts die Anzahl der Apotheken in der Stadt auf vier erhöht hatte, beschloss der Rat 1453 mit einer Arzneitaxe die Preisgestaltung der Arzneien genau festzulegen ${ }^{561}$. Die Mitarbeit der gelehrten Mediziner an derartigen Anordnungen lässt sich aber erst seit dem Ende des 15. Jahrhunderts deutlich beobachten. Am 28. Juni 1490 ersuchten die Augsburger Ratsherren die Räte in Ulm und Nürnberg in einem Schreiben um einen Arzt, der sie bezüglich der vorzunehmenden Regelung der hiesigen Apotheken unterweisen könnte. Im

\footnotetext{
558 MARTZ, Gesundheitswesen, S. 61-62; STEIN, Franzosenkrankheit, S. 149. In der von Elisabeth Martz bearbeiteten Zusammenstellung der Ehefrauen von Augsburger Doktoren im 16. Jahrhundert sind 14 Frauen aus altem Adel und Patriziat, 5 aus dem reichen Bürgertum und 3 aus den Familien von Akademikern nachgewiesen. ${ }^{559}$ StEIN, Franzosenkrankheit, S. 155; FleISCHMANN, Ärztefamilie, S. 27-29; RoTH, Reformation I, S. 343, 352, 360 .

${ }^{560}$ GensthaleR, Medizinalwesen, S. 52-57, 72-77.

561 Seit 1283 gab es in Augsburg eine Apotheke, die von der patrizischen Familie Hofmair geleitet wurde. Erst mit dem Aufkommen des zweiten Apothekers Meister Peter im Jahr 1417 entstand Konkurrenz auf dem Arzneimittelmarkt. Bis zum Jahr 1457 wurde ein Apotheker vom Rat besoldet, was in der Folgezeit vermutlich wegen der Einrichtung mehrerer Apotheken nicht mehr notwendig war, um die Medikamentenversorgung in der Stadt sicherzustellen.
} 
Anschluss daran wurde 1491 eine erneuerte Arzneitaxe vom Rat erlassen ${ }^{562}$. Einige Jahre später, genauer gesagt im Jahr 1512, visitierten die Augsburger Ärzte zusammen mit den Ratsverordneten die Apotheken in der Stadt und bereiteten auch eine neue Version der Arzneitaxe vor $^{563}$. In derselben Verordnung wurde auch der Handel mit stark treibenden Heilmitteln ausschließlich auf die vom Rat eingeschworenen Apotheker beschränkt, indem sich die Mediziner und die Krämer nicht mehr an einem solchen Geschäft beteiligen sollten $^{564}$.

Der eigentliche Aufstieg gelehrter Ärzte zu städtischen Medizinexperten fand aber erst während der Pestepidemie im Jahre 1521 statt. Mit einem gemeinsam verfassten Schreiben an den Rat legten die vier Stadtärzte ihre medizinischen Anleitungen vor, auf dessen Grundlage die städtische Pestpolitik in Augsburg organisiert wurde ${ }^{565}$. Diese erfolgreiche Beratung der Doktoren führte dazu, dass sie auch bei anderen Regelungen des städtischen Medizinalwesens eine wichtige Rolle spielten. Als typisch für die Folgezeit ist nämlich zu beobachten, dass die gelehrten Mediziner kollektiv die Initiative ergriffen, um den Rat auf Missstände im städtischen Gesundheitswesen aufmerksam zu machen und gleichzeitig Vorschläge zu deren Abhilfe zu unterbreiten. Mit dieser Art von Eingaben wandte sich jeweils die gesamte Gruppe von angestellten Stadtärzten an den Rat, wodurch die Mediziner anscheinend das erforderliche Gewicht vor der städtischen Obrigkeit wirksamer erreichen konnten.

Die vermehrte Einflussnahme der gelehrten Mediziner auf die gesundheitspolitischen Anordnungen des Rates zeigte sich schon kurz nach dem Abklingen der Pest. In dem erwähnten Schreiben von 1521 wiesen die Stadtärzte am Rande auf die notwendige Reorganisation des Blatterhauses hin ${ }^{566}$. Sie warnten vor allem vor der Verwendung der

\footnotetext{
${ }^{562} \mathrm{Ob}$ ein Arzt aus Ulm bzw. Nürnberg zur Beratung des Apothekenwesens damals nach Augsburg kam, ist aber leider nicht überliefert.

${ }^{563}$ Die erste Apothekenvisitation in Augsburg, die von einem nicht näher genannten artzat durchgeführt werden sollte, ist schon für das Jahr 1417 bezeugt, als Meister Peter die zweite Apotheke in der Stadt eröffnete, dazu in: GENSTHALER, Medizinalwesen, S. 54.

${ }^{564}$ Darunter verstand man vor allem die innerlich wirkenden Heilmittel, wie z.B. Theriak, die damals zur arzneilichen Purgation des Körpers von überflüssigen Säften und schädlichen Stoffen verwendet wurde.

${ }^{565}$ Siehe S. 135-137. Obwohl es keine Quellenangaben überliefert sind, kann man eine derartige Beratung gelehrter Ärzte schon während der Pestepidemie von 1494 annehmen, als damals die ersten Maßnahmen nach den Grundsätzen der medizinischen Pestlehre vom Rat eingeführt wurden. Um diese Zeit waren die hoch angesehene Doktoren wie Adolph Occo I., Johann Jung oder Ambrosius Jung in Augsburg tätig.

${ }^{566} \mathrm{Zu}$ diesen Veränderungen in der Organisation des städtischen Blatterhauses in den Jahren 1521/22: STEIN, Franzosenkrankheit, S. 141-152. Ergänzend zur Interpretation von Claudia Stein muss man hier bemerken, dass die Reform des Blatterhauses nicht nur im allgemeinen Zusammenhang mit der Neuregelung des Armenwesens in Augsburg steht, sondern direkt auf die seit der Pestepidemie von 1521 stärkere Einflussnahme von gelehrten Medizinern auf die gesundheitspolitischen Regelungen des Rates zurückzuführen ist. Die Verbesserungsvorschläge zur Tätigkeit des Blatterhauses reichten die Stadtärzte nämlich im Jahr $1521 \mathrm{mitsamt}$
} 
Quecksilbersalbe bei der Behandlung der sogenannten Franzosenkrankheit, da deren unsachgemäßer Gebrauch die Erkrankung verschlimmere bzw. zu dauerhaften Schäden bei den Patienten führen könne. Als Alternative zu dieser umstrittenen Kur priesen sie die Anwendung eines anderen Arzneimittels an, ohne jedoch die Einzelheiten der neuen Therapie preiszugeben. Ihrer Ansicht nach könnten die Kranken damit wirkungsvoller behandelt und die im Blatterhaus anfallenden Kosten beträchtlich gesenkt werden. Der Rat reagierte im Jahr 1522 auf die Vorschläge der Mediziner, indem er erneuerte Blatterhausordnung erließ. Darin wurde ein neues Heilmittel, das Guaiacumholz verordnet, das nach einheiliger Meinung der Doktoren alle bis dahin verwendeten Arzneien gegen die Franzosenkrankheit bei weitem übertreffen sollte. Diese standardisierte Therapie wirkte sich auch auf die Anzahl der im Haus beschäftigten Heiler aus, die nun von fünf auf zwei, einen Doktor und einen Wundarzt, beschränkt wurde. Die neue Blatterhausordnung von 1522 regelte auch die Organisation des Hauses, indem man das Verhältnis zwischen den dortigen Medizinern genau festlegte. Der Aufgabenbereich des Wundarztes wurde strikt auf die Behandlung der äußeren Krankheitserscheinungen eingeschränkt. Der Wundarzt sollte bei der Ausübung seiner Tätigkeit auch durch den gelehrten Mediziner kontrolliert werden. Dadurch erreichten die gelehrten Mediziner eine weitgehende Aufsicht über die Behandlungsverfahren im Blatterhaus und stellten seither die unangefochtene Leitungsspitze dieser Institution dar.

In den Zwanziger Jahren des 16. Jahrhunderts hatten die Doktoren auch das Mitspracherecht bei der Hebammenprüfung erlangt ${ }^{567}$. Ihre Kontrolle konnten sie im Jahre 1531 indirekt ausweitern, als das städtische Hebammenwesen unter die Aufsicht von vier bis sechs „Obersten Frauen“ gestellt wurde. Diese stammten aus der Augsburger Führungsschicht und waren entweder mit einflussreichen Ratsmitgliedern oder mit gelehrten Ärzten verheiratet, wie z.B. die Ehefrau von Doktor Gereon Sailer, Felicia. Damit nutzten die Augsburger Doktoren auch ihre familiären Bindungen zur weiteren Einflussnahme auf das städtische Medizinalwesen geschickt aus.

Umfassende gesundheitspolitische Regelungen wurden vom Rat im Jahre 1532 erlassen, als die vier Augsburger Stadtärzte Johann Trenklin, Adolph Occo II., Johann Tiefenbach und Gereon Sailer weitere Reformvorschläge vorlegten ${ }^{568}$. Die größten

den Anweisungen zur städtischen Pestbewältigung ein, worauf 1522 die Blatterhausordnung vom Rat erlassen wurde. StAA, CM IV, Nr. 7 (1521): Schreiben der vier Augsburger Stadtärzte.

${ }^{567}$ HAMmond, Civic Health Care, S. 99ff, 181-192; STEIN, Franzosenkrankheit, S. 155.

${ }^{568}$ Am 4. Juni 1532 wurden zunächst die allgemeinen Vorschläge zur Ordnung des städtischen Medizinalwesens von vier Augsburger Stadtärzten an den Rat gerichtet, die in einem Schriftstück mit der späteren Vermerkung „Der Arzt querelen“ zusammengefasst wurden. Dieses Schreiben wurde dann an die Ärzte mit der Bitte zurückgeleitet, ihre Beschwerden exakter zu formulieren. Am 11. Juni legten die Doktoren wieder ihre gesundheitspolitischen Vorschläge beim Rat vor, die nun in acht Punkten verfasst waren. Der Antrag wurde nach 
Beschwerden der Doktoren betrafen die ungehinderte Tätigkeit von frembden Artzt in Augsburg, die mit ihren Behandlungsverfahren zahlreiche Schäden unter der Bevölkerung anrichteten. Darunter waren von den Stadtärzten vornehmlich ungelernte Heiler gemeint, die sich ohne obrigkeitliche Kontrolle mit der Heilbehandlung betätigten: muller, pfaffen, Juden, Cristen, weib und manns personnen, so sich bisher zu Arzneyen understannden ${ }^{569}$. Zur Eindämmung derartiger Kurpfuscherei verordnete der Rat daher, dass keine fremden Ärzte ohne Vorwissen der Bürgermeister ihre Arzneikunst in Augsburg ausüben dürften. Die städtischen Doktoren beanspruchten dabei ein Mitspracherecht bei der obrigkeitlichen Zulassung der fremden Heiler, welches ihnen jedoch vom Rat noch nicht zuerkannt wurde ${ }^{570}$. Bezüglich der herumziehenden Ärzte machten sie den Rat auch darauf aufmerksam, dass besonders viele Franzosenkranke in der Stadt durch die unprofessionelle Quecksilberbehandlung fremder Frantzosen Aerzte und landfarer völlig verderbt wurden ${ }^{571}$. Diese unter den gelehrten Medizinern umstrittene Schmierkur, die im Blatterhaus mit der erneuerten Hausordnung von 1522 eingestellt wurde, war jedoch oftmals die einzige Therapiemöglichkeit, die von den Armen bezahlt werden konnte. Aus diesem Grund baten die Stadtärzte den Rat dafür Sorge zu tragen, dass die Quecksilberschmierkur in der Stadt nur den hiesigen Barbieren erlaubt sein möge. Die Doktoren wiesen dabei darauf hin, dass die handwerklichen Mediziner der sachen genug geschickt sein und ihre Lebensgrundlage durch die vielen fremden Heiler auf Augsburgs Straßen und Märkten äußerst beeinträchtigt werde. Die Durchführung dieser umstrittenen Therapie wollten jedoch die gelehrten Mediziner noch selbst kontrollieren und zuvor, wie bei der Behandlung mit Guaiacumholz im Blatterhaus, eine notwendige Purgation der Kranken anordnen. Mit der darauf im Sinne der Doktoren erfolgten Regelung des Rates wurde den gelehrten Medizinern ein weitgehendes Aufsichtsrecht über die Heilverfahren der Franzosenkrankheit in der Stadt zuerkannt. Im Bezug auf das städtische Apothekenwesen wurde dagegen in dieser Ratsverordnung die unbeaufsichtigte Medikamentenvergabe durch Apothekerdiener untersagt und ein oder

dem Verlesen an vier Ratsverordnete weitergeleitet, die aufgrund der Anregungen von Stadtärzten die vorzunehmenden Regelungen herausarbeiteten. Zum Schriftverkehr von 1532 zwischen den Doktoren und dem Rat in: Gensthaler, Medizinalwesen, S. 78-79. Das Schreiben der Stadtärzte vom 11. Juni und die darauf unternommenen Verordnungen des Rates in: StAA, CM IV, Nr. 11 (11. Juni 1532).

569 Darüber hinaus zeigten die Doktoren beim Rat auch einige Heiler in Augsburg an, deren medizinische Tätigkeit sich als besonders schädlich erwiesen hatte: der Jud hab vill leut betrogen, Mer der pfaff Sailler, der auch ain verderber viller menschen bißher geweßt, wie dann von Maister Petter Balbierer, vor unnser frauen thor sesßhaft, vill erfaren mucht werden, Mer muller.

${ }^{570}$ Das weitgehende Aufsichtsrecht über alle in Augsburg tätigen Mediziner, darunter auch über Bader, Barbiere und Wundärzte, erreichten die Doktoren erst mit der Errichtung des Collegium Medicum im Jahre 1582, dazu in: MARTZ, Gesundheitswesen und Ärzte im 16. Jahrhundert, S. 7-10; DIENINGER, Das Collegium Medicum Augustanum, S. 1239-1243; SAlZBRunN, Das Ende des Augsburger Collegium medicum, S. 156-162.

${ }^{571}$ Dazu vgl. in: STEIN, Franzosenkrankheit, S. 156-157. 
zweimal pro Jahr eine Apothekenvisitationen von den Ratsverordneten und Doktoren angeordnet. Der Rat bekräftigte erneut das Verbot des Handels mit purgierenden Arzneien außerhalb der Apotheken und erließ eine erneuerte Arzneitaxe ${ }^{572}$.

Darüber hinaus wollten die Stadtärzte auch mehr Einfluss auf die allgemeine Ausübung der Wundarzneikunst in der Stadt gewinnen. Den gelehrten Ärzten war vor allem daran gelegen, die Trennung zwischen der inneren gelehrten und der äußeren handwerklichen Medizin strikter einzuhalten ${ }^{573}$. Demnach beanspruchten sie die alleinige Herrschaft über den inneren Körper und versuchten die Handwerkschirurgen aus diesem Bereich möglichst zu verdrängen bzw. zu beaufsichtigen. Auf Begehr der Ärzte wurde daher vom Rat verordnet, dass die Barbiere ohne vorherige Beratung durch die Doktoren keine schwerkranken Menschen mehr zur Ader lassen dürften. Dabei wurde auch hervorgehoben, dass zu den Aufgaben der Wundärzte vornehmlich die Behandlung der äußeren Schäden am Körper gehören solle. Die Forderung der Stadtärzte, keine purgierenden Arzneimittel ohne die Aufsicht der gelehrten Ärzte anzuwenden, wurde aber nicht durchgesetzt. Der Rat richtete eine diesbezügliche Anfrage an das Barbierhandwerk, worauf dieses mit einem eigenem Appell antwortete: solange sich die gelehrten Ärzte nicht völlig aus dem Bereich der äußeren Medizin heraushielten, wollten die Barbiere auf die Anwendung der innerlich wirkenden Arzneien auch nicht verzichten. Die Doktoren führten auch selbst - je nach Gelegenheit wundarzneiliche Eingriffe durch, worauf z.B. die von Bernhard Schludin gebrauchte Selbstbezeichnung der beider Leibs- und Wundarznei Doktor eindeutig hinweist ${ }^{574}$. Weil aber die gelehrten Ärzte eine solche Zusage anscheinend nicht abgeben wollten, folgte ihrer Aufforderung an den Rat damals keine obrigkeitliche Regelung ${ }^{575}$.

Als Reaktion auf die Beschwerden der Stadtärzte über die Missstände im Augsburger Medizinalwesen erließ der Rat 1533 die erste Wundarzneiordnung ${ }^{576}$. Zur besseren Kontrolle

\footnotetext{
${ }^{572}$ Die Stadtärzte nannten in ihrem Schreiben an den Rat auch diejenige Personen, die sich mit derartigem unerlaubtem Arzneihandel in Augsburg betätigten: Claus Ehinger soll bißher als Zeltlin und Coliguinten, und annder Artzney verkaufft haben, das ganz schedlich sey, Deßgleichen die Zanprecher, in: StAA, CM IV, Nr. 11 (11. Juni 1532).

573 Zur damaligen Vorstellung von der Zweiteilung des menschlichen Körpers vgl. in: STEIN, Franzosenkrankheit, S. 156-167.

${ }^{574}$ StAA, CM IV, Nr. 24: Supplikation von Doktor Schludin an den Rat (aus dem Inhalt für 1535 datierbar).

${ }^{575}$ Die Verabreichung der purgierenden Arzneimittel von Handwerkschirurgen wurde erst im Jahre 1568 per Ratsverordnung verboten, was auch auf die Initiative der Doktoren erfolgte. Wegen des darauf erhobenen Widerspruches von Wundärzten wurden Anfragen über die entsprechenden Regelungen nach Ulm und Nürnberg geschickt, woraus sich ergab, dass in Nürnberg ein solches Verbot schon verhängt worden war. Wohl aufgrund dieser auswärtigen Erkundigungen sowie einer Empfehlung der Ratsverordneten über die Apothekenvisitation erließ der Rat endgültig ein entsprechendes Verbot, wovon aber der Wundt und Faal tranckh zum Austreiben des geronnenen Blutes ausgenommen wurde. Obwohl die Zuständigkeiten bezüglich des Verabreichens von treibenden Arzneien seither obrigkeitlich geregelt waren, kam es in der folgenden Zeit noch zu einigen Streitigkeiten zwischen Wundärzten und Doktoren, dazu in: DIEMINGER, Bader, S. 112-116.

${ }^{576}$ Die Wundarzneiordnung von 1533 ist editiert und erläutert in: DIEMINGER, Bader, S. 54-60, I-V.
} 
der Handwerkschirurgen in Augsburg verordnete man, dass sich keine Barbiere oder Bader mit der Wundarznei oder dem Aderlass betätigen dürften, ohne sich vorher von sechs eingeschworenen Meistern des Handwerks prüfen und sich zugleich vom Rat bestätigen zu $\operatorname{lassen}^{577}$. In dieser Ordnung ist auch die steigende Einflussnahme von Doktoren auf die wundarzneilichen Heilverfahren erkennbar. Bei der Strafandrohung von einem halben Gulden sollte der Aderlass seitdem nur noch an jenen Tagen durchgeführt werden, die von den Stadtärzten zuvor festgelegt worden waren. Zu Beginn eines jeden Jahres errechneten nämlich die eingeschworenen Doktoren astrologisch günstige Aderlasstage, die danach den Badern und Barbieren mitgeteilt wurden. Außerdem wurde den handwerklichen Medizinern nicht erlaubt, ohne die vorherige Beratung eines Doktors die schwerkranken Menschen zur Ader zu lassen. Die Ausnahme von dieser Regel galt nur in der pestilentz, offenbar wegen der Notwendigkeit der möglichst schnellen Ausführung von therapeutischen Maßnahmen und der großen Anzahl von Betroffenen in dieser Zeit ${ }^{578}$.

Die vier Stadtärzte traten während der Pestepidemie von 1535 wieder als Medizinexperten auf ${ }^{579}$. Mit ihrer gedruckten Pestschrift wollten sie vor allem die Obrigkeit zum Ergreifen weiterer Maßnahmen gegen die Kurpfuscherei in der Stadt bewegen. Gleichzeitig erhoben sie auch einen Expertenanspruch unter allen Medizinern in Augsburg, indem sie sowohl andere gelehrte Ärzte als auch die Wundärzte zur Beachtung der galenischhippokratischen Grundsätze bei der Behandlung der Pestkranken aufforderten. Nach der festen Überzeugung der Augsburger Stadtärzte sollte die Medizinkunst strikt auf der Lehre der antiken Autoren beruhen. Abgesehen von ihrem Misstrauen gegen zu starke Anlehnung an die Schriften von arabischen Medizinern lehnten sie vor allem die grundlegenden Erneuerungen in der Medizinlehre ab. Damit ist vor allem die damals sehr umstrittene Lehrmeinung von Theophrast von Hohenheim gemeint, der besser als Paracelsus bekannt ist $^{580}$. Dieser gelehrte Arzt kritisierte den Stand der zeitgenössischen Medizin und stellte die Zuverlässigkeit der antiken Viersäftelehre in Frage. Die Paracelsischen Ansichten wurden deshalb von den meisten zeitgenössischen Medizinern heftig kritisiert, weshalb seine Ideen keine größere Verbreitung fanden. Gleichermaßen geschah dies auch in Augsburg, als Johannes Talhauser, einer der hiesigen Stadtärzte, die umstrittenen Auffassungen des

\footnotetext{
${ }^{577}$ Die Verdrängung von ungeprüften Heilern aus Augsburg konnte anscheinend mit der Wundarzneiordnung von 1533 nicht völlig umgesetzt werden, worauf 1535 eine diesbezügliche Ratsverordnung erlassen wurde, in: StAA, CM IV, Nr. 14: Verordnung gegen die unaprobierten Wundärzte (4. Juli 1535).

${ }^{578}$ Zur Bedeutung des Aderlasses bei der Therapie von Pestkranken, siehe S. 117-120.

${ }^{579}$ Siehe S. 65-68.

${ }^{580}$ Zur Lehre von Paracelsus u.a. in: PAGEL, Paracelsus; WEEKS, Paracelsus.
} 
medizinischen Reformators Paracelsus öffentlich unterstützte ${ }^{581}$. Im Jahr 1536 erschien in Augsburg die Druckschrift „Die Grosse Wundarzney“ des Paracelsus, der ein Brief von Talhauser vorangestellt wurde ${ }^{582}$. Darin hatte er in Einklang mit Paracelsus einen Tiefstand der gelehrten Medizin festgestellt und dessen Bemühungen um eine allgemeine Erneuerung der Heilkunst entschieden begrüßt. Durch ein solches Auftreten ihres Berufsgenossen fühlten sich die anderen Doktoren in Augsburg persönlich angegriffen. Unter Vermittlung des Rates zwangen sie Talhauser daher zu einer öffentlichen Entschuldigung, die in Form einer gedruckten „Apologia“ von ihm abgefasst werden solle. Außerdem musste er auch dafür sorgen, dass sein umstrittener Brief in den nächsten Auflagen der „Wundarzney“ nicht wieder abgedruckt wurde.

Auf diese Kontroverse um Talhauser bezieht sich offensichtlich auch die Druckschrift „Conclusiones et propositiones universam Medicinam, per genera comprehendentes“ aus dem Jahr 1538, die von allen in Augsburg tätigen Doktoren: Ambrosius Jung, Adolph Occo II., Johann Tieffenbach, Gereon Sailer, Damian Behem, Bernhard Schludin und Johann Vogt zusammengestellt wurde ${ }^{583}$. Die Ärzte unterstrichen darin ausdrücklich, dass sie niemals von den galenisch-hippokratischen Grundsätzen der Medizinlehre abweichen würden. Anschließend legten sie die Regeln zur Durchführung sachgemäßer Behandlungen dar und wiesen noch auf die Notwendigkeit weiterer Verbesserungen im Augsburger Apothekenwesen hin. Angesichts des Streites mit den anderen Doktoren verließ Talhauser Augsburg noch im selben $\mathrm{Jahr}^{584}$.

\footnotetext{
${ }^{581}$ Zur Kontroverse um Doktor Johannes Talhauser, in: TELLE, Talhauser; auch in: STEIN, Franzosenkrankheit, S. 153-154; GENSTHALER, Medizinalwesen, S. 95-97.

582 PARACELSUS, Der grossen Wundartzney/ das Erst (ander) Buch [Augsburg: Heinrich Steiner 1536].

583 Jung/OCCO/TIEFFEnBACH/SAILER/BEHEM/SCHLudi/Vogt, Conclusiones et propositiones [Augsburg: Philipp d. Ä. Ulhart 1538].

${ }^{584}$ Er zog danach nach Tübingen und diente dort seit 1538 Herzog Ulrich als Leibarzt und diplomatisch tätiger Rat. In den Jahren 1540-1544 nahm er auch eine Lehrtätigkeit an der medizinischen Fakultät der Universität Tübingen auf. Im Jahr 1544 kehrte Talhauser wieder nach Augsburg zurück, wo er ohne städtische Anstellung seine medizinische Praxis weiter ausübte. Während der Epidemie von 1547/48 stellte Talhauser die Pestschrift von Doktor Matthias Böham von Neuhaus zum Neudruck zusammen, indem er dessen Text noch um einen weiteren Teil ergänzte. Er starb 1548 in Augsburg. Zur erwähnten Pestschrift, siehe S. 68-70.
} 


\section{Kommunalisierung der Armen- und Krankenfürsorge}

Seit dem Ende des 15. Jahrhunderts kann man also eine steigende Einflussnahme gelehrter Mediziner auf die obrigkeitlichen Regelungen des Medizinalwesens beobachten. Dass die Augsburger Stadtherren auf die Vorschläge der Stadtärzte mehrheitlich auch mit entsprechenden Verordnungen reagierten, war die Folge der sich vollziehenden Umwandlungen in der Reichstadt um 1500. Die Entwicklung der städtischen Pestpolitik steht nämlich im engen Zusammenhang mit der sich damals abschließenden Kommunalisierung der Armen- und Krankenfürsorge in Augsburg ${ }^{585}$. In der Forschung wird damit ein Prozess des allmählichen Übergangs der diesbezüglichen Zuständigkeiten von kirchlichen Institutionen auf die städtischen Räte bezeichnet. Ursprünglich gehörte die karitative Fürsorge zu den ausschließlichen Angelegenheiten der Kirche, deren Anfänge in Augsburg mit der Gründung des Heiligen-Kreuz-Hospitals im 10. Jahrhundert bezeugt sind ${ }^{586}$. Als rein kirchliche Stiftung war auch das Heilig-Geist-Hospital zwischen 1239 und 1245 vor dem Roten Tor errichtet worden, das von einer nach den Regeln des Heiligen Augustinus lebenden Laienbruderschaft unter Aufsicht des Augsburger Bischofs geführt wurde. In dieser großen Hospitaleinrichtung, die am Ende des 14. Jahrhunderts in den Pfarrbezirk Ulrich und Afra innerhalb der Stadtmauern verlegt wurde, versorgte man mehr als 250 alte und chronisch kranke Bürger ${ }^{587}$. Auch das erstmals 1264 erwähnte Siechenhaus bei der Kirche des Heiligen Servatius, in dem eine Gemeinschaft von Aussätzigen wohnte, unterstand den kirchlichen Machtträgern ${ }^{588}$. Seit dem Ende des 13. Jahrhunderts lässt sich aber deutlich eine verstärkte Anteilnahme der Bürgerschaft im Wohltätigkeitsbereich erkennen. Mit religiös motivierten Stiftungen für Arme und Kranke versuchten die Bürger zunehmend ihr ewiges Seelenheil zu sichern. Für diese frommen Zwecke spendeten sie vor allem Geld, Lebensmittel und Kleider an Hospitäler oder direkt an die Bedürftigen in der Stadt. In den Quellen werden diese Spenden als selgerät,

\footnotetext{
${ }^{585}$ Zur Augsburger Armen- und Krankenfürsorge in: BISLE, Armenpflege; CLASEN, Armenfürsorge (1-2); DERS., Arm und Reich; KIEßLING, Gesellschaft, S. 159-175, 215-235; LENGLE, Spitäler; SEIDA UND LANDSBERG, Historisch-statistische Beschreibung; STEIN, Franzosenkrankheit, S. 95-100; WERNER, Stiftungen. Allgemein zu diesem Thema vor allem in: SACHßE/TENnstedT, Geschichte; REICKE, Spitalwesen; JÜTTE, Armenfürsorge; FISCHER, Städtische Armut; BULST/SPIEß, Sozialgeschichte mittelaterlicher Hospitäler.

${ }^{586}$ Das Heiligen-Kreuz-Hospital war eine Einrichtung des Domstifts, zu dessen Erstausstattung von einem Bürger namens Walgar der Ort Margertshausen zum Unterhalt für zwölf arme Menschen gestiftet wurde. Bischof Ulrich wies zu diesem Zweck um 955 ein Gebäude unterhalb der Stadtmauer an. Um Mitte des 12. Jahrhunderts wurde dieses Hospital in die Vorstadt verlegt und seine Verwaltung von den AugustinerChorherren von Hl. Kreuz übernommen, KIEßLING, Gesellschaft, S. 160; LENGLE, Spitäler, S. 202.

${ }^{587}$ LENGLE, Heilig-Geist-Spital, S. 156; DERS., Spitäler, S. 202; HöRMANN, Heilig-Geist-Hospital, S. 145-176.

${ }^{588}$ KIEßLING, Gesellschaft, S. 168-169; LENGLE, Spitäler, S. 203. Im Jahre 1288 wurde diese Einrichtung erweitert, als das Ehepaar Langenmantel ein weiteres Haus für acht Sieche stiftete.
} 
gotberat oder jartag bezeichnet ${ }^{589}$. Darüber hinaus wurden auch von der reichen Bürgerschaft die sogenannten Seelhäuser speziell für arme Frauen oder kleine Hospitäler gestiftet, die zur Versorgung bestimmter Personengruppen vorgesehen waren ${ }^{590}$. Im Jahre 1440 entschloss sich das Kramerehepaar Konrad und Afra Hirn zur Stiftung eines Pilgerhauses in der Jakobervorstadt, in welchem vier Betten für Pilger auf dem Weg zu den Walfahrtsorten Rom, Compostela und Loretto bereitgestellt wurden ${ }^{591}$. Auf die Initiative der Familie von Lorenz Egen geht das 1445 gegründete Antonspital zurück, in das zwölf alte und arme Männer aus dem Handwerk aufgenommen werden sollten ${ }^{592}$.

Mit der gesteigerten Stiftungstätigkeit der Bürgerschaft machte sich auch bald der Einfluss des Rates auf das Wohlfahrtswesen in der Stadt bemerkbar. Die mildtätigen Güter und Gelder für die Fürsorgeeinrichtungen wurden von den Bürgern unter die Aufsicht der städtischen Pflegschaften gestellt, die sich allmählich zu regulären Ratsämtern entwickelten. Durch die Einsetzung der Pfleger konnte der Rat daher schon am Ende des 13. Jahrhunderts ein Mitspracherecht in den ursprünglich rein kirchlichen Institutionen erlangen und damit ihre Verwaltung weitgehend mitbestimmen ${ }^{593}$. Außerdem gründeten die Ratsherren auch selbst Hospitäler in der Stadt. Im Jahr 1348 wurde in der sich entwickelnden Vorstadt vor dem Sträfinger Tor das Jakobshospital erbaut, das zur Versorgung für arme Pilger und Kranke sowie verarmte Ratsmitglieder und Bürger vorgesehen war ${ }^{594}$. In der ersten Hälfte des 15. Jahrhunderts wurden dagegen zwei städtische Siechenhäuser zur Aufnahme Aussätziger eingerichtet: St. Sebastian vor dem Jakobertor und St. Wolfgang an der Wertachbrücke ${ }^{595}$. Alle diese Institutionen waren rein städtische Gründungen, die von Anfang an durch vom Rat ernannte Pfleger verwaltet wurden. Zu diesem Zeitpunkt wurde jedoch die Versorgung von Armen und Kranken in Augsburg noch nicht entscheidend vom Rat finanziert und durchgeführt. Die überwiegende Form der fürsorglichen Maßnahmen wurde von Stiftungen

\footnotetext{
${ }^{589}$ LENGLE, Spitäler, S. 205; HerBERGER, Seelhäuser, S. 283-296; KIEßLING, Gesellschaft, S. 219-225.

${ }^{590}$ Ein Überblick der von der Bürgerschaft gestifteten Seelhäuser für die armen Bürgerinnen im Spätmittelalter, in: KIEßLING, Gesellschaft, S. 225-230; HERBERGER, Seelhäuser, S. 283-296.

${ }^{591}$ Trometer, Pilgerhaus; BisLe, Armenpflege, S. 110; STEIN, Franzosenkrankheit, S. 108-109. Das Pilgerhaus (später auch Almosenhaus genannt), stand seit den 1520er Jahren unter der direkten Verwaltung von Almosenherren und diente seither zur Versorgung der Kranken, die mit heilbaren Gebrechen und Schäden befallen waren. Diese Einrichtung wurde 1578 mit einer großzügigen Stiftung des Augsburger Bürgers Martin Zobel versehen und an die Barfüßergräben verlegt. So konnten in der zweiten Hälfte des 16. Jahrhunderts ungefähr siebzig Kranke dort versorgt werden.

${ }^{592}$ LENGLE, Spitäler, S. 202.

593 Zur Bedeutung der städtischen Pflegschaften in der Einflussnahme des Rates auf die Verwaltung des Heiligen-Geist-Hospitals und des St. Servatius Siechenhauses, in: KIEßLING, Gesellschaft, S. 159-175.

${ }^{594}$ LENGLE, Spitäler, S. 202; KIEßLING, Gesellschaft, S. 173-174, 231.

${ }^{595}$ KIEßLING, Gesellschaft, S. 169-173; STEIN, Franzosenkrankheit, S. 96, 109-112. Während in St. Sebastian vornehmlich 15 weibliche Kranke untergebracht waren, versorgte man in St. Wolfgang die gleiche Anzahl Aussätzigen beiderlei Geschlechts.
} 
aus der wohlhabenden Bürgerschaft geleistet, so dass die karitative Fürsorge bis weit in das 15. Jahrhundert hinein zum größten Teil auf privater und kirchlicher Initiative beruhte ${ }^{596}$.

Die weitgehende Kommunalisierung der Armen- und Krankenfürsorge vollzog sich erst schrittweise seit dem Ende des 15. Jahrhunderts, was vornehmlich mit der allgemeinen Entwicklungslinie der Stadt zusammenhängt. Mit der in den 1470er Jahren einsetzenden wirtschaftlichen Aufschwungsphase stieg Augsburg zur Handels- und Finanzmetropole Oberdeutschlands auf und dank der Verbindungen zum Haus Habsburg unter der Regentschaft Maximilians I. (1486/96-1519) wuchs die Reichsstadt in europäische Dimension hinein $^{597}$. Damit war der starke Ausbau der städtischen Administration verbunden, so dass sich bis zur Jahrhundertwende etwa 40 Ratsämter herausbildeten und eine Reorganisation der Exekutive (Scharwächter, Stadtknechte, Gassenhauptleute) durchgeführt wurde ${ }^{598}$. Der Rat erlangte damals auch eine weitgehende Verwaltungsautonomie. Seine Stellung gegenüber dem Bischof als zweitem Bezugspunkt der ehemals geteilten Stadtherrschaft verstärkte sich, zumal die entscheidenden Kompetenzen für die Gerichts- und Finanzhoheit an die Bürgerschaft übergangen waren. Als Konsequenz dieser Entwicklung verlegte Bischof Friedrich von Zollern 1486 seine bevorzugte Residenz nach Dillingen. Außerdem wurde durch die regelmäßige Wiederbesetzung der städtischen Spitzenämter mit denselben Bürgern die Ausbildung einer semiprofessionellen politischen Führungsgruppe gefördert. Aufgrund dieser vielseitigen Umwandlungen bildete sich folglich auch ein neues Verständnis der Ratsherrschaft heraus, die immer stärker als eine von der Gemeinde abgehobene Obrigkeit definiert wurde. Der Rat leitete dabei ihre Autorität vornehmlich vom Kaiser und Gott ab und legitimierte sein Handeln gegenüber der Bürgerschaft mit dem Begriff des „Gemeinen Nutzens“. Damit war auch die allgemeine Intensivierung der Ratsherrschaft verbunden, die vor allem als eine Reaktion der Stadtbehörden auf die sozialen Veränderungen anzusehen ist. Die rasche Entwicklung der Stadt löste einen enormen Zustrom von Menschen aus, so dass sich die Bevölkerung Augsburgs innerhalb von vier Jahrzehnten - von 1490 bis 1530 - von ca.

\footnotetext{
${ }^{596}$ KIEßLING, Gesellschaft, S. 230-231; RoGGE, Für den Gemeinen Nutzen, S. 216-217.

${ }^{597}$ Zur allgemeinen Entwicklung Augsburgs um 1500: KIEßLING, Augsburg; RoGGE, Für den Gemeinen Nutzen, S. 99-105; ZORN, Augsburg, S. 166-168. Die erste wirtschaftliche Wachstumsphase erfolgte in Augsburg schon in der ersten Hälfte des 15. Jahrhunderts, als die neu entwickelte Barchantproduktion für die Entwicklung des Textilsektors sorgte. Danach herrschte jedoch seit 1448/50 andauernde Depression in der Wirtschaft, die erst seit den 1470er Jahren mit der Entwicklung der neuen wirtschaftlichen Leitsektoren überwunden werden konnte. Die zweite Wachstumsphase basierte dabei auf dem in zahlreichen Handelsgesellschaften organisierten Fernhandel, auf dem Bank- und Darlehensgeschäft sowie auf dem Engagement von Augsburgern im Tiroler und Ungarischen Bergbau. Trotz dieser wirtschaftlichen Expansion war jedoch die Augsburger Wirtschaftsstruktur nach wie vor stark von den Handwerkern geprägt. Zur Entwicklungslinie der Augsburger Wirtschaft: KIEßLING, Wirtschaft; KELLENBENZ, Wirtschaftsleben; SCHREMMER, Wirtschaftsmetropole.

598 Zur Veränderung der Ratsherrschaft in Augsburg am Ende des 15. Jahrhunderts vgl. vor allem die einschlägige Studie: RogGE, Für den Gemeinen Nutzen; dazu auch KIEßLING, Augsburg; SIEH, Stadtverfassung.
} 
18.000 auf etwa 35.000 Personen verdoppelte ${ }^{599}$. Durch einen derartig großen Bevölkerungszuwachs veränderte sich die soziale Struktur der Stadt, woraus folglich viele Probleme für die Stadtgemeinde entstanden. Die harte ökonomische Lage zahlreicher Zuwanderer konnte nicht beträchtlich verbessert werden, weil die großen Gewinne aus dem wirtschaftlichen Aufschwung überwiegend Teilhabern oder Besitzern der großen Handelsgesellschaften zugute kamen. Die Augsburger Vermögen konzentrierten sich am Anfang des 16. Jahrhunderts noch stärker als zuvor auf wenige Steuerzahler. Im Jahre 1516 kontrollierten 6,5\% der reichsten Bürger fast $90 \%$ des gesamten zu versteuernden Vermögens in der Stadt, während die auf ca. 80 \% der Bevölkerung geschätzte Unterschicht nur $1,3 \%$ des Steuervermögens besa $\beta^{600}$. Eine direkte Folge dieser sozialen Entwicklung war die zunehmende Polarisierung in arm und reich, zudem waren zahlreiche Einwohner durch die ständige Mangelernährung in ihrer Existenz bedroht. Der Anteil von Bedürftigen in Augsburg, die regelmäßig auf Almosen zur Sicherung ihres Lebensunterhalts angewiesen waren, wird in der Forschung auf $10 \%$ der gesamten Stadtbevölkerung geschätzt, was in dieser Zeitperiode ca. 3.000 Menschen in Augsburg betroffen haben dürfte ${ }^{601}$. Zu diesem Kreis sind vor allem arme Gesellen und Tagelöhner aus dem Textilgewerbe, Witwen, Waisenkinder, Behinderte und Kranke zu zählen. Die Existenzgrundlage der Armen verschlechterte sich weiter, wenn in der Stadt Epidemien ausbrachen oder Missernten zu Teuerungen von Lebensmitteln führten ${ }^{602}$. Aus diesen Gründen ergriff der Rat seit der zweiten Hälfte des 15. Jahrhunderts viele Maßnahmen zur städtischen Armenfürsorge, um während der raschen Stadtentwicklung und bei all ihren sozialen Konsequenzen die bestehende Ordnung aufrechterhalten zu können ${ }^{603}$. Der Rat versuchte einerseits mit den entsprechenden Verordnungen die Bettelei in der Stadt möglichst einzuschränken und unter strengere Kontrolle zu nehmen. Andererseits verfolgte der Rat eine zunehmend organisierte Versorgungspolitik mit Lebensmitteln, d.h. in Zeiten von Missernten und Teuerung wurde subventioniertes Korn verkauft oder kostenlos Brot an die Armen verteilt. Zu diesem Zweck

\footnotetext{
599 JAHN, Einwohnerzahl, S. 394; DERS., Sozialstruktur, S. 188.

${ }^{600}$ Wie sich diese Wachstumsphase der Augsburger Wirtschaft auf die einzelnen sozialen Schichten auswirkte und wie extrem ungleich sich dabei die Gewinne verteilten, belegen anschaulich die folgenden Statistiken, die anhand der Angaben des gesamten städtischen Steuervermögens zusammengestellt sind: 1475: Oberschicht: 3,5\% Bevölkerung - 70\% Stadtvermögen, Mittelschicht: 13,2\% Bevölkerung - 25,5\% Stadtvermögen, Unterschicht 83,3\% Bevölkerung - 4,5\% Stadtvermögen; 1516: Oberschicht: $6,5 \%$ Bevölkerung - 89,9\% Stadtvermögen, Mittelschicht: 15,6\% Bevölkerung - 8,8\% Stadtvermögen, Unterschicht: 77,9\% Bevölkerung 1,3\% Stadtvermögen. RoGGE, Für den Gemeinen Nutzen, S. 102; GEFFCKEN, Schichtung, S. 218.

${ }^{601}$ KießLing, Gesellschaft, 217, 234; DerS., Augsburg, S. 246; RogGe, Für den Gemeinen Nutzen, S. 213-216. Eine ähnliche Größenordnung von Bedürftigen von etwa fünf bis zehn Prozent der Gesamtbevölkerung wird auch für Freiburg i. Br. und Straßburg um 1500 angenommen, in: FISCHER, Armut, S. 57-58.

${ }^{602}$ Der sprunghafte Anstieg der Getreidepreise ist in Augsburg für die folgenden Jahre nachweisbar: 1489-1491, 1496, 1500-1502, 1509-1511, 1515-1517, 1519, in: RoGGE, Für den Gemeinen Nutzen, S. 103.

${ }^{603}$ ClASEN, Armenfürsorge (1-2); RogGE, Für den Gemeinen Nutzen, S. 218-230.
} 
wurden spezielle Kornhäuser zur Anlage von städtischen Getreidevorräten errichtet und der Getreidehandel und Brotverkauf von den Stadtbehörden verstärkt kontrolliert. Das gesamte Armenwesen wurde mit der Gründung des Almosenamtes im Jahr 1522 vom Rat neu geordnet und für die regelmäßige Verteilung von Almosen an die Bedürftigen ein spezielles städtisches Fürsorgesystem eingerichtet ${ }^{604}$. Mit der modifizierten Almosenordnung von 1541 verhängte der Rat auch ein allgemeines Bettelverbot in Augsburg ${ }^{605}$. In diesem Zusammenhang ist auch die städtische Krankenfürsorge mit der Errichtung der Pesthäuser sowie des Blatterhauses am Ende des 15. Jahrhunderts zu deuten. Diese Einrichtungen waren vornehmlich für die medizinische Versorgung von Armen in der Stadt vorgesehen ${ }^{606}$. Unter den ärmeren Einwohnern waren insbesondere die Dienstleute auf die städtische Pflege angewiesen, weil sie im Krankheitsfall oft ohne Hilfe zurückgelassen wurden. Insbesondere während der Pestepidemien war die Not aufgrund der großen Anzahl armer Menschen so groß, dass eine besondere obrigkeitliche Lösung dringend geboten war $^{607}$. Die Kommunalisierung der Armen- und Krankenfürsorge erreichte ihren Höhepunkt nach der offiziellen Einführung der Reformation in Augsburg im Jahr 1537, als durch die Vertreibung des katholischen Klerus und die Auflösung der noch bestehenden Klöster und Stifte die Verantwortung für das gesamte städtische Fürsorgewesen in die Hände des Rates gelangte ${ }^{608}$. Auch nach der Rekatholisierung der Stadt durch Kaiser Karl V., die nach der Niederlage des von Augsburg unterstützten Schmalkaldischen Bundes im Jahre 1548 durchgeführt wurde, verblieben die ehemaligen kirchlichen Hospitäler weiterhin in den Händen des Rates. Die städtische Obrigkeit verwaltete folglich eine ganze Reihe von Einrichtungen, die zur Behandlung spezifischer Krankheitsbilder bestimmt waren ${ }^{609}$. Auch wenn im 16. Jahrhundert einige neue

\footnotetext{
${ }^{604}$ Nach wie vor existierte aber die traditionelle Wohltätigkeit von Seiten der Kirche sowie bürgerliche Stiftungen für Armen, wie z.B. die zwischen 1514-1523 von Jakob Fugger gestiftete Sozialsiedlung, die sogennante Fuggerei, dazu in: TIETZ-STRÖDEL, Fuggerei.

${ }^{605}$ Siehe S. 170-171.

${ }^{606}$ Zur Bestimmung der Zielgruppe für die Augsburger Pesthäuser, siehe S. 129 und 142; Über die Aufnahmekriterien im Augsburger Blatterhaus, in: STEIN, Franzosenkrankheit, S. 118-119.

607 Auch in anderen großen Städten im deutschsprachigen Gebiet wurden auch in der Frühen Neuzeit Pesthospitäler - vor allem als obrigkeitliche Reaktion auf die infolge der enormen Bevölkerungsentwicklung ebenfalls stark anwachsende Armut - gegründet. Genauso wie in Augsburg waren diese geräumigen Einrichtungen in erster Linie für arme Dienstboten und Fremde geplant. In den kleineren und weniger bevölkerungsreichen Städten, die über keine finanziellen Mittel zur Erbauung eines speziellen Pesthospitals verfügten, wurden dagegen für einen befristeten Zeitraum einige dazu geeignete Häuser genutzt oder hölzerne Hütten am Rande der Stadt errichtet, dazu in: UlBRICHT, Pesthospitäler, S. 96-118.

${ }^{608}$ STEIN, Franzosenkrankheit, S. 100; ROTH, Reformationsgeschichte, Bd. 2, S. 290ff; IMMENKRÖTER, Reformation und Parität, S. 400-403.

${ }^{609}$ Zur Spezialisierung der städtischen Krankeneinrichtungen in Augsburg, in: STEIN, Franzosenkrankheit, S. $105-115$.
} 
private Stiftungen wie z.B. die Fuggerschen Holz- und Schneidhäuser gegründet wurden, so war in dieser Zeit die kommunale Form der Krankenfürsorge in Augsburg vorherrschend ${ }^{610}$.

\section{Zusammenfassung}

Die vier Augsburger Stadtärzte verfassten gemeinsam im Jahr 1535 eine Druckschrift, um aufgrund der damaligen Wissensbestände zu erklären, was die Pestilentz an jr selbs sey. Dieses Ziel verfolgte auch die vorliegende Studie, in der das Phänomen der Pest in der Reichstadt Augsburg um 1500 in ihrem zeitgenössischen Kontext analysiert wurde. Diesem Forschungsvorhaben lagen zwei methodologische Überlegungen zugrunde.

Zum Ersten wurde die Möglichkeit der retrospektiven Diagnose der Pest in der historischen Forschung hinterfragt. In der Mehrzahl der bisherigen Studien wird implizit angenommen, dass sich die moderne Krankheitseinheit Pest, die nach ihrem Auslöser, dem Bakterium Yersinia pestis definiert ist, auch in den Zeiten vor der Entstehung der mikrobiologischen Wahrnehmungsweise in der zweiten Hälfte des 19. Jahrhunderts identifizieren und zurückverfolgen lasse. Demnach wären die historischen Seuchenbilder, die mit dem Namen „Pest“ von den Zeitgenossen bezeichnet wurden, mit dieser modernen Krankheitseinheit gleichzusetzen. In dieser Annahme bleibt aber unbeachtet, dass solche Schilderungen keine medizinische Datensammlung darstellen, die zuverlässige Hinweise auf einen bestimmten Erreger im Sinne der modernen Bakteriologie liefern können. Im vormikrobiologischen Zeitalter bestanden andere Erklärungsmodelle für Seuchen, die nach den damaligen kulturspezifischen Wahrnehmungsmöglichkeiten formuliert wurden und mit den Erkenntnissen der heutigen Medizinwissenschaft unvereinbar sind. Eine retrospektive Diagnose im Sinne der modernen Krankheitseinheit ist daher ausschließlich im Rahmen der Paläopathologie möglich, indem man in organischem Material mit molekulargenetischen Verfahren bakterielle DNA nachweist. Aus diesen Gründen wurde die retrospektive Diagnose der Pest in der vorliegenden Arbeit abgelehnt. Bei der Analyse der schriftlichen Quellen

\footnotetext{
${ }^{610}$ Zur Durchführung der wundarzneilichen Behandlungen, wie z.B. die Kurierung von Brüchen oder die Entfernung von Steinen wurden 1540 von den Fuggern das Schneidehaus am Krautmarkt und 1583 das große Schneidehaus am Rossmarkt gegründet, dazu in: DIEMINGER, Bader, S. 131-139; Zur Behandlung der Franzosenkrankheit stiftete man dagegen um 1525 das Holzhaus in der Fuggerei und 1560 das Holzhaus auf dem Gänsbühl, dazu in: STEIN, Franzosenkrankheit, S. 128-139. Einschlägig zu Stiftungen Jakob Fuggers des Reichen: SCHELLER, Memoria an der Zeitwende.
} 
musste die „Pest“ immer diejenige Krankheit bleiben, die von den Zeitgenossen nach den damaligen Kategorien als Pest wahrgenommen wurde. Im Zusammenhang damit wurde auch der problematische Versuch unterlassen, die modernen Erkenntnisse auf das damalige Seuchengeschehen zu projizieren, um den Verlauf der früheren Epidemien nach dem heute gültigen medizinischen Maßstab zu deuten.

Zum Zweiten wurde in der vorliegenden Studie das von Martin Dinges herausgearbeitete Modell zur Erforschung der „sozialen Konstruktion von Seuchen“ angewendet. Dieser Forschungsansatz basiert auf der Vorstellung, dass sowohl die Krankheit selbst als auch die Methoden zu ihrer Bewältigung in einer Gesellschaft jeweils konstruiert werden. Dies geschieht in einem komplexen Prozess der Interaktionen zwischen vier Gruppen von sozialen Akteuren: 1.) Kranke in einem sozialen Umfeld, 2.) Heiler mehr oder minder professioneller Art, 3.) Obrigkeiten oder Staaten, die für autoritative Regelungen und deren Durchsetzung in einer Gesellschaft zuständig sind und 4.) „an Seuchen Interessierte“, wie die Kirche oder andere Interessensgruppen, die in erster Linie nicht durch eine Heilfunktion definiert sind. In einer Wechselbeziehung mit der „Umwelt““ werden Diskurse und Praktiken von sozialen Akteuren entwickelt, die wiederum zu „öffentlichen Meinungen“ werden und in einem historisch variablen Machtgefälle zum Einsatz kommen.

Für die spezielle Ausrichtung der vorliegenden Untersuchung wurde dieses Forschungsmodell entsprechend modifiziert. Bei der Erforschung der Pest ist vor allem festzuhalten, dass die verschiedenen sozialen Akteure keinesfalls als homogene Gruppen anzusehen sind, sondern dass sich diese in sehr differenzierten, vielschichtigen Gruppen bewegen. Die Grenzen zwischen diesen vier Gruppen sind häufig auch als fließend zu betrachten. So konnte etwa die Erkrankung an der Pest oder schon die potentielle Ansteckungsgefahr während der Epidemien bedeuten, dass eine und dieselbe Person gleichzeitig verschiedenen Gruppen zugehörig war.

Die sozialen Akteure wurden in dieser Arbeit auch bezüglich ihrer Relevanz im Prozess der sozialen Konstruktion der Pest stärker differenziert. Seit der Entwicklung der obrigkeitlichen Pestpolitik, die den Umgang mit der Seuche in der gesamten Stadtgemeinde weitgehend prägte, gilt die städtische Obrigkeit als ein besonders gewichtiger Akteur. Darüber hinaus ist zu konstatieren, dass das soziale Aushandeln öffentlicher Meinungen innerhalb einer beschränkten Anzahl von Denkweisen stattfinden konnte, weil in jeder Epoche immer nur eine bestimmte Zahl von Diskursen existiert. Die damalige Vorstellungswelt über die Pest ließ sich dabei in zwei Diskurse, den religiösen und den medizinischen einordnen, die in einer engen Wechselbeziehung zueinander standen und im Laufe der Zeit einigen Veränderungen unterlagen. In diesem Zusammenhang ist jedoch hervorzuheben, dass die auf dem 
zeitgenössischen Wissensstand über die Pest beruhenden Praktiken gleichzeitig auch von verschiedenartigen Handlungsmotivationen und Interessen der sozialen Akteure beeinflusst werden konnten.

Die Hauptthese der Arbeit war es, dass sich mit der Entwicklung der obrigkeitlichen Pestpolitik am Ende des 15. Jahrhunderts die soziale Konstruktion der Pest in Augsburg herausbilden konnte, deren Grundlage von einem engen Zusammenspiel des religiösen und medizinischen Diskurses geprägt war. Im Zentrum der Untersuchung stand die erkenntnisleitende Frage, auf welche Weise sich diese zwei Diskurse in der Reichstadt Augsburg verbreiteten und damit die Seuchenbewältigung in der Stadtgemeinde bestimmten. Der Zeitrahmen der durchgeführten Analyse umfasste die Zeitperiode seit den ersten in Augsburg belegten Pestepidemien in der zweiten Hälfte des 14. Jahrhunderts bis zur Etablierung der städtischen Pestbewältigung in der Mitte des 16. Jahrhunderts. Im gesamten Untersuchungszeitraum war eine Vielfalt von Quellenbegriffen für die Pest anzutreffen, die von den Zeitgenossen in verschiedenen lexikalischen Varianten verwendet wurden. Dieses breite Begriffsspektrum lässt sich in drei semantische Gruppen einteilen. Zur ersten Gruppe gehört die „Pestilentz“, die in der nach dem Ausbruch der ersten Epidemie in Europa von gelehrten Medizinern formulierten Pestlehre als ein zentraler Begriff fungierte. Der zweite gebräuchliche Name war „Brechen“, womit vornehmlich sichtbare Krankheitserscheinungen auf dem Körper, z. B. die Pestbeulen, bezeichnet wurden. Als dritte Gruppe von den in Augsburg geläufigen Pestbegriffen ist die Bezeichnung „großes Sterben“ zu nennnen. Mit diesem sprachlich am vielfältigsten fixierten Quellenausdruck wird primär ein krankheitsbedingtes Massensterben betont. In der Analyse der Augsburger Quellenbegriffe war festzustellen, dass „Pestilentz“, „Brechen“ und „großes Sterben“ von den Zeitgenossen synonym verwendet wurden. Eine beschränkte Differenzierung in ihrer Gebrauchspraxis konnte man nur im Hinblick auf die ausgewerteten Quellengattungen erkennen.

Anhand der chronikalischen Überlieferung wurden die Grundzüge der Wahrnehmung und Bewältigung der Pest im spätmittelalterlichen Augsburg herausgearbeitet. Dabei fiel vor allem die fundamentale Rolle des religiösen Diskurses auf, der die Handlungen der Zeitgenossen während der Pestzeit am stärksten steuerte. In der Erscheinung der Pest sah man die übernatürliche Wirkungskraft Gottes schlechthin und verankerte ihren Ursprung im religiösen Kontext. Die ausgebrochenen Epidemien wurden als göttliche Strafe für begangene Sünden gedeutet, welche man nur durch den Einsatz religiöser Mittel abwenden kann. Zum üblichen Maßnahmenkatalog der Zeitgenossen gehörten Bittprozessionen, Votivmessen, Fastenzeiten, Gebete zu Schutzpatronen wie dem Heiligen Sebastian oder dem Heiligen 
Rochus, wie auch die Errichtung verschiedenartiger Stiftungen für die Kirche. Darüber hinaus wurden die ersten Epidemien vornehmlich als ,großes Sterben“ wahrgenommen, wodurch die übliche Vorstellung der Pest als einer Krankheit mit sehr hohen Todeszahlen zum Ausdruck gebracht wurde. Die hohe Virulenz der Pest war - zeitgenössisch betrachtet - einer der relevantesten Unterschiede zu anderen im Spätmittelalter auftretenden Krankheiten. Die in den spätmittelalterlichen Chroniken verzeichneten Todesstatistiken sollten dabei als anschauliche Hervorhebung der ungewöhnlich hohen Sterblichkeit in der Pestzeit und nicht als exakte Wiedergabe der Opferzahlen angesehen werden.

Angesichts der Seuchengefahr stellte damals die Flucht in die nicht von der Pest befallenen Nachbarorte eine wichtige Handlungsoption für die Zeitgenossen dar. Die Möglichkeit der Pestflucht beanspruchten nicht zuletzt die Stadtherren bis 1438 völlig uneingeschränkt. Vor diesem Hintergrund ist vorauszusetzen, dass die Bewältigung der Pest im spätmittelalterlichen Augsburg nur in bescheidenem Ausmaß von der Obrigkeit verwaltet werden konnte. Die überlieferten Quellenbelege über die Aktivität der Stadtherren beziehen sich dabei ausschließlich auf die Bestattung von Pestopfern. Der Rat bemühte sich nämlich darum, vor allem für die armen Stadtbewohner möglichst viele Gemeinschaftsgruben auf den Kirchhöfen innerhalb der Stadt zur Verfügung zu stellen. Wie der Konflikt mit dem Augsburger Bischof Anshelm von Nenningen im Jahr 1420 deutlich aufzeigte, konnten solche städtischen Maßnahmen aufgrund des Widerstands der Kirche zumindest temporär verhindert werden. Dieser Vorfall enthüllt einerseits die beschränkten Handlungsmöglichkeiten der städtischen Obrigkeit während der Pestzeit, andererseits bestätigt er die Vorherrschaft des religiösen Diskurses im Spätmittelalter, dessen mächtiger Träger die Kirche war.

In der Augsburger Überlieferung konnten keine Angaben über die medizinischen Praktiken während der spätmittelalterlichen Epidemien gefunden werden, obwohl die grundlegenden Strukturen des Medizinalwesens schon damals bestanden. Es ist jedoch mit Sicherheit davon auszugehen, dass der medizinische Diskurs zu diesem Zeitpunkt nur eine untergeordnete Rolle spielte. Die Gründe dafür sind vielfältig, u.a. die medizinische Fürsorge in der Pestzeit war alleinige Aufgabe der Familie und Haushaltsmitglieder und zudem nur solchen Bürgern zugänglich, die einen Arzt auch bezahlen konnten. Die Armen waren daher von der ärztlichen Betreuung weitgehend ausgeschlossen bzw. auf die Behandlung nicht ausgebildeter Heiler angewiesen. Außerdem ist die Verbreitung der medizinischen Pestlehre innerhalb breiterer Bevölkerungsschichten im Spätmittelalter als gering einzuschätzen. Die städtische Obrigkeit erließ auch keine Verordnungen, die alle Stadtbewohner dazu anhielten, sich entsprechend 
den damaligen medizinischen Grundsätzen zu verhalten. Im spätmittelalterlichen Augsburg waren daher die Doktoren bzw. Wundärzte die alleinigen Träger der so genannten Pestlehre.

Die Verbreitung medizinischen Wissens außerhalb des angestammten Gelehrtenkreises setzte in Augsburg erst mit den Pestschriften von gelehrten Ärzten ein, die seit den Siebziger Jahren des 15. Jahrhunderts gedruckt wurden. Auf dem Augsburger Buchmarkt wurde seither eine große Auswahl von deutschsprachigen Schriften angeboten, die in verschiedenen Druckformen von Einblattdrucken, über Regimina sanitatis mit einem Abschnitt über die Pest, bis hin zu selbständigen Werken über die Pestlehre erschienen. Aus diesem umfangreichen Schrifttum, das sowohl von Augsburger wie auch von auswärtigen Doktoren abgefasst wurde, konnten die Stadtbewohner ihren Vermögensverhältnissen oder ihrem Bildungsniveau entsprechend geeignete Druckschriften erwerben. Viele dieser Texte wurden dabei nicht nur während der Pestepidemien in Augsburg, sondern häufig auch beim Ausbruch von Seuchen in anderen Orten aufgelegt. Die Wiederauflagen einiger Bücher bestätigen ebenfalls ihre große Beliebtheit unter den Zeitgenossen. In den medizinischen Pestdrucken aus dem Zeitraum bis 1550 wird vorrangig der gemeine man als erwünschter Leser angesprochen. Das Hauptziel dieser deutschsprachigen Pestschriften bestand also darin, breiten Bevölkerungsschichten die notwendige Aufklärung über die Seuche zu vermitteln. Diese meist praxisorientierten Gebrauchsschriften weisen bei Form und Einteilung des Textstoffes zahlreiche Gemeinsamkeiten auf. Abgesehen von den medizinischen Pestblättern, die in kurzer und einheitlicher Form einen grundsätzlichen Maßnahmenkatalog gegen die Pest darlegen, lassen sich in den meisten Druckschriften deutlich drei zusammenhängende Grundteile über die Ätiologie, Prophylaxe und Therapie unterscheiden.

Als theoretische Grundlage der damaligen Pestlehre galt das antike Medizinkonzept von den vier Säften, die in einem dauerhaften Mischungsverhältnis für jeden Menschen individuell festgelegt waren und seine körperliche Konstitution bestimmten. Krankheiten wurden demnach als ein widernatürlicher Zustand des Körpers gedeutet, der vornehmlich durch eine verderbliche Veränderung der Körpersäfte ausgelöst wurde. Der zeitgenössischen Medizin zufolge lag das Wesen der Pest in einer giftigen Materie begründet, die in den Körper eindrang und eine rasche Vergiftung des Blutes und aller Flüssigkeiten verursachte. Dadurch wurde die Zusammensetzung aller körperlichen Säfte gänzlich zerstört und infolgedessen erhob sich das sogenannte pestilenzialische Fieber, das zumeist von äußerlichen Krankheitserscheinungen wie Beulen oder Blattern begleitet wurde. Die gelehrten Mediziner waren aber nicht imstande zu erklären, was für ein Stoff genau für die Entstehung der Pest verantwortlich sei und worin sich die allgemeinen und wagen Erkenntnismöglichkeiten in der 
vormikrobiologischen Ära widerspiegeln. In allen Traktaten wiesen sie hingegen daraufhin, dass der menschliche Körper diese giftartige Substanz vor allem aus der Luft empfange. Über den Ursprung dieses Phänomens wurden verschiedene Erklärungen abgegeben, die sich vor allem auf astronomische und natürliche Ursachen bezogen. Eine derartige Luftvergiftung könnte durch ungünstige Konstellationen der Planeten, schädliche Auswirkungen unregelmäßig verlaufender Jahreszeiten sowie durch Fäulnisvorgänge und Ausdünstungen von stillstehenden Gewässern, unbegrabenen Leichen, Abfallstoffen oder anderen faulenden Dingen mit üblem Geruch ausgelöst werden. Nach der damaligen Vorstellung verlor die Luft aufgrund dieser verschiedenartigen Vorgänge ihre gesunde Beschaffenheit, weshalb in Folge dessen, eine Pestepidemie entstehen konnte.

Für die Ärzte war es auch offensichtlich, dass die Pest von einem Kranken leicht an einen Gesunden weitergegeben werden konnte. In allen analysierten Traktaten bis 1550 wurde aber noch keine zusammenhängende Ansteckungslehre dargelegt. Die Erkenntnis des Infektionsphänomens war für die Mediziner nur bei der praktischen Pestbewältigung relevant und wurde in den theoretischen Ausführungen nicht näher erörtert. Aufgrund der in den Pestschriften verstreuten Hinweise kann man jedoch feststellen, dass die Ansteckung vor allem im Zusammenhang mit den Vorstellungen über die Luftvergiftung gedeutet werde. Nach der zeitgenössischen Wahrnehmung verdarben vergifteter Atem sowie alle anderen von dem Kranken ausgehenden Stoffe gleich den gennanten anderen Faktoren die Luft, wodurch andere Menschen die giftige Materie in sich aufnehmen konnten. Deswegen könnte die Ansteckung sowohl im direkten Kontakt mit dem Kranken wie auch mit den von ihm gebrauchten Gegenständen, darunter insbesondere solche aus Tuch, erfolgen. Auf diese Weise erklärten die Doktoren die epidemische Verbreitung der Pest.

Die Analyse der Augsburger Pestschriften zeigte deutlich, dass die medizinische Pestlehre stark von der Religion beeinflusst war. Abgesehen von den rein astrologischen Ausführungen Alexander Seitz', der den Ausbruch der Pestepidemien ausschließlich mit den primären Auswirkungen himmlischer Einflüsse zu erklären versuchte, war die Ätiologie der Pest allgemein im religiösen Diskurs der Zeit eingebettet. Der Auffassung der damaligen Mediziner zufolge war sowohl der Ursprung wie auch die Abwendung einer Seuche letztlich allein von Gottes Verhängnis abhängig. Daher wurden die Zeitgenossen in den Pestschriften ausdrücklich zur Ausübung der religiösen Praktiken ermahnt. Dabei sollten insbesondere die Pestheiligen Sebastian und Rochus, wie auch die Gottesmutter Maria um ihre spezielle Fürsprache bei Gottvater angerufen werden. Die wichtige Rolle des Heiligenkultes belegen anschaulich die streng religiösen Pestblätter, die von Augsburger Druckern aufgelegt wurden. 
Im Zusammenhang mit der Heiligenverehrung lassen sich aber spätestens seit den Dreißiger Jahren des 16. Jahrhunderts die reformatorischen Einflüsse in den Augsburger Pestschriften deutlich erkennen. In der protestantischen Auslegung des Fürsprechens wurden die Gottesmutter Maria und die üblichen Pestheiligen wie z.B. Sebastian oder Rochus völlig fallengelassen und die Bedeutung von Christus in den Vordergrund der religiösen Pestbewältigung gerückt.

In den Pestschriften stellten die gelehrten Ärzte zahlreiche Anweisungen zur Prophylaxe zusammen, die auf den zeitgenössischen Anschauungen über die Ätiologie der Pest basierten und ihre praktische Umsetzung im Alltag ausführlich erläuterten. Unter anderem wurden Schutzmaßnahmen erörtert, die eine Ansteckung mit der Pest verhindern sollten. Außerdem wurden vorbeugende Mittel zum Reinigen der Luft sowie Maßnahmen für eine gesunde Lebensführung erklärt. Daneben empfahlen die Ärzte verschiedene Prophylaktika für den täglichen Gebrauch sowie, unter ihrer persönlichen Beratung und Betreuung, eine individuelle Reinigung des Körpers von überschüssigen Flüssigkeiten. Obwohl in den medizinischen Schriften eine große Auswahl an Prophylaxemaßnahmen geboten wurde, galt der Rückzug aus dem verpesteten Ort als sicherstes Mittel gegen die Pest. In der Reformationszeit wurde aber diese Anschauung der Mediziner durch die Schriften protestantischer Theologen maßgeblich beeinflusst und verändert. Vor einer Flucht in die gesunde Luft der Umgebung sollten demzufolge alle Gläubigen gründlich erwägen, ob sie nicht statt dessen verpflichtet wären, ihren Verwandten, Freunden und Nachbarn Hilfe zu leisten oder ihrem geistlichen oder weltlichen Amt nachzugehen. Andererseits sollten alle Menschen, die während der Pestepidemie vor Ort ausharren mussten, mit allen zur Verfügung stehenden medizinischen Mitteln für ihre eigene Gesundheit sorgen. Ohne diese Pflege würde man nicht nur sich selbst schaden, sondern durch die eigene Vernachlässigung auch viele andere Personen mit der gefährlichen Krankheit vergiften. Auch damit könnten die Menschen gegen das Gebot der christlichen Nächstenliebe verstoßen und die ganze Gemeinde in Todesgefahr bringen. Die Anwendung medizinischer Maßnahmen wurde von der Reformationsbewegung theologisch legitimiert.

In der damaligen Medizinlehre wurde auch ein kohärentes Heilverfahren für die Pestkranken ausgearbeitet. Die medizinische Behandlung bestand vornehmlich darin, dass man die giftige Materie möglichst schnell aus dem erkrankten Körper auszutreiben versuchte. Die Reinigung vom Pestgift sollte durch die Abgabe von Blut, Schweiß, das Öffnen von Pestbeulen und Ausscheidungen wie Harn und Stuhlgang erfolgen. Zu diesem Zweck machten sich die Mediziner alle bewährten Heilmethoden der damaligen Medizin zunutze: Aderlass, 
Arzneimittel und Wundarznei, die in einer bestimmten Abfolge angewendet werden sollten. Die Mediziner nahmen die Pest als die gefährlichste Krankheit jener Tage wahr, hielten aber ihre erfolgreiche Bewältigung für möglich. Die Wirkungskraft medizinischer Maßnahmen werde aber letztlich allein durch göttlichen Willen bedingt. Diese zeitgenössische Vorstellung ist keinesfalls als Ausdruck der Ratlosigkeit gegenüber der schwer heilbaren Krankheit zu interpretieren, sondern ergibt sich logischerweise aus der religiös geprägten Pestlehre.

In der vorliegenden Arbeit wurde nachgewiesen, dass die städtische Obrigkeit erst während der Pestepidemie 1494 die ersten Maßnahmen ergriffen hatte, welche den medizinischen Grundsätzen entsprachen. An einem entlegenen Ort an der Stadtmauer wurde der St. Stephan Gottesacker für die Bestattung der Pestopfer angelegt. Die Begräbnisse auf zwei Kirchöfen im Stadtzentrum durften nicht mehr stattfinden und der städtische Raum sollte zum ersten Mal vor der Verunreinigung durch schädliche Ausdünstungen aus den überfüllten Massengräbern geschützt werden. In der Jakobervorstadt wurden zwei spezielle Häuser eingerichtet, die für die medizinische Versorgung der Armen unter den Pestkranken vorgesehen waren. Eines dieser Häuser wurde bereits im Jahr 1495 in ein städtisches Blatterhaus umgewandelt, in welchem man vorrangig Personen unterbrachte, die an der sogenannten Franzosenkrankheit litten, welche sich $\mathrm{zu}$ dieser Zeit rasch in Augsburg verbreitete. Die Gründung der neuen Friedhofsanlage und der Pesthäuser musste der Rat mit den kirchlichen Machthabern wegen der neuen Regelungen der Seelsorge und der Opfergaben in der Pestzeit vereinbaren.

Im Jahr 1521 änderte der Rat die städtische Pestpolitik dahingehend, dass diese unmittelbar an den Anweisungen der Augsburger Stadtärzte ausgerichtet wurde und diesen Folge leistete. Die Ratschläge der gelehrten Mediziner in den Mittelpunkt rückend, wurde eine neue Hospitalanlage außerhalb der Stadt errichtet. Diese sogenannten Brechhäuser waren vornehmlich für die medizinische Versorgung der Armen in der Pestzeit vorgesehen. Für die finanzielle und organisatorische Administration der Häuser ernannte der Rat zwei Pfleger, die gleichzeitig die Aufsicht über das städtische Blatterhaus versahen. Die administrative Verknüpfung dieser Institutionen ging dabei aus der früheren Organisation der städtischen Pestfürsorge hervor, als die Franzosen- und Pestkranken während der Epidemien vor 1521 noch gemeinsam in zwei Häusern in der Jakobervorstadt untergebracht waren. Die alltägliche Verwaltung der Häuser befand sich vor Ort in den Händen des Brechvaters, dem zahlreiche Dienstleute zur Verfügung standen. Zur medizinischen Behandlung der Kranken wurden die beim Rat angestellten Barbiere und Stadtärzte verordnet. Der Rat erließ zudem auch die ersten Anordnungen darüber, wie sich städtischen Bewohner während der Pest zu verhalten hatten. 
Diese sehr konkreten Handlungsanweisungen, z.B. eine Verordnung zum Schutz vor der weiteren Verbreitung der Seuche und zur Reinhaltung des städtischen Raums, waren bei Androhung empfindlicher Geldstrafen von allen zu beachten.

Die damals vom Rat ergriffenen Maßnahmen bildeten seither die Grundlage der städtischen Pestpolitik, welche bis zur Mitte des 16. Jahrhunderts weiter entwickelt wurden. Im Vordergrund standen vor allem Anordnungen zur wirksameren Infektionsvorbeugung im städtischen Raum. Zu diesem Zweck wurde 1535 eine völlige Quarantäne der Kranken und Rekonvaleszenten eingeführt, so dass sie sich in ihren Häusern aufhalten mussten und bei Strafe des sofortigen Verweises aus der Stadt ihre Häuser nicht verlassen durften. Der Höhepunkt derartiger Vorsorgemaßnahmen wurde während der Pestepidemie in den Jahren 1547-1548 erreicht, als unter Kaiser Karl V. der Reichstag in Augsburg stattfand. Alle Pestkranken mussten - dem kaiserlichen Befehl nach - mitsamt ihrer Kleidung in die städtischen Brechhäuser gerbracht und dort versorgt werden. Die Mitbewohner der infizierten Personen sollten dagegen in ihren Häusern einer 14-tägigen Quarantäne unterliegen oder für diese Zeit die Stadt verlassen. Diese stark restriktive Tendenz ist auch in anderen Punkten der aus Anlass des Reichstages erlassenen Pestordnung deutlich zu erkennen. So wurden beispielsweise die angedrohten Geldstrafen im Vergleich zu vorhergehenden Verordnungen verdoppelt.

Darüber hinaus beschloss der Rat angesichts der bestehenden Pestgefahr im Jahr 1533 einen außerstädtischen Gottesacker anzulegen, um die städtische Gemeinde vor schädlichen Ausdünstungen aus den Gräbern zu schützen. Diese Maßnahme setzte man unter heftigem Widerstand der katholischen Kirchenherren um. Mit der Gründung des sogenannten Oberen Gottesackers wurde die Schließung von zwei innerstädtischen Kirchhöfen als Begräbnisplätze angeordnet, wodurch deren Pfarrer nicht nur in ihren Pfarrechten beeinträchtigt wurden, sondern auch mit einer Minderung ihres aus den Bestattungen resultierenden Einkommens rechnen mussten. Die Idee der Friedhofsverlegung, die ursprünglich auf die Pestlehre von Medizinern zurückzuführen ist, wurde seit ihrer eifrigen Befürwortung durch Martin Luther mit der Reformation im Allgemeinen assoziiert. In der Begräbnisordnung von 1535 wurden generell alle innerstädtischen Begräbnisse in der Pestzeit verboten und die Pestopfer seither ausschließlich auf zwei Gottesäckern außerhalb der Stadt bestattet.

Im Zusammenhang mit der städtischen Pestpolitik sind auch die Regelungen zur Eindämmung des Bettelwesens in der ersten Hälfte des 16. Jahrhunderts zu bewerten. Die zeitgenössische Vorstellung von Bettlern als eine potentielle Seuchenquelle konnte - neben der in der 
Forschung immer wieder angeführten Einflussnahme der Reformation und der Zentralisierung der Armenfürsorge - die städtische Organisation des Armenwesens ebenso bestimmen.

Die fundamentale Umwandlung in der sozialen Konstruktion der Pest, die sich in der Reichstadt Augsburg seit dem Ende des 15. Jahrhunderts vollgezogen hatte, ergab sich aus der historischen Verflechtung verschiedener Faktoren. Die Voraussetzung für den kulturellen Wandel im Umgang mit der Pest war die Entwicklung einer neuen Wissensordnung, die sich mit der schrittweisen Verbreitung und Rezeption der medizinischen Vorstellungen etablierte. Dieser Prozess setzte in Augsburg offensichtlich mit der Popularisierung der Pestlehre mit Hilfe gedruckter Schriften ein, wodurch ein breit angelegter Hintergrund zur Durchsetzung des medizinischen Diskurses in nahezu allen Bevölkerungsschichten entstehen konnte. Am wichtigsten war jedoch der Aufstieg gelehrter Mediziner zu städtischen Experten, wodurch die gesundheitspolitischen Entscheidungen des Rates von diesen maßgeblich mitgestaltet werden konnten. In der zweiten Hälfte des 16. Jahrhunderts ergriffen die Augsburger Stadtärzte kollektiv die Initiative, um den Rat auf Missstände im städtischen Gesundheitswesen aufmerksam zu machen und gleichzeitig Vorschläge zu deren Abhilfe vorzulegen. Aufgrund ihrer Aufforderungen wurden damals alle wichtigen Bereiche des städtischen Medizinalwesens weitgehend neu bzw. erstmalig geregelt: das Apothekenwesen, die Behandlung der Franzosenkrankheit, die Prüfung der Hebammen, die Wundarzneikunst, die Kontrolle ungelernter Heiler und nicht zuletzt die städtische Pestbewältigung. Für die erfolgreiche Durchsetzung ihrer Richtlinien war von Bedeutung, dass viele Augsburger Doktoren schon seit dem Ende des 15. Jahrhunderts zu größtem Ansehen in ihrer medizinischen Tätigkeit gelangt waren sowie eine hohe soziale Stellung und enge verwandtschaftliche Beziehungen zur städtischen Führungsschicht erreicht hatten. Die bei vielen Medizinern beliebten Studienaufenthalte an italienischen Universitäten leisteten einen wesentlichen Beitrag zur Rezeption der dort gelehrten medizinischen Sachverhalte. Ebenso führten sie dazu, dass die gesundheitspolitischen Handlungsmuster und die institutionelle Pestbewältigung aus den italienischen Städten rezipiert wurden und später in Augsburg Anwendung fanden.

In der vorliegenden Studie wurde deutlich aufgezeigt, dass die städtische Pestpolitik in einem engen Zusammenhang mit der zu einem Ende gelangenden Kommunalisierung der Armenund Krankenfürsorge steht. Der wirtschaftliche Aufschwung Augsburgs um 1500 war von einer raschen Steigerung der Bevölkerungsanzahl begleitet, deren direkte Folge eine starke Polarisierung im städtischen Sozialgefüge war. Beim Ausbruch der Epidemien war insbesondere die existenzielle Grundlage der Armen bedroht. Aus diesen Gründen musste der 
Rat entschiedene Maßnahmen ergreifen, um die bestehende Ordnung in der Stadtgemeinde während der Pestzeit aufrechterhalten zu können. Mit der sich damals entwickelnden Pestpolitik des Rates konnte der medizinische Diskurs unter der Gesamtbevölkerung in Augsburg weit verbreitet werden. Dafür waren vornehmlich zwei Neuerungen maßgebend: Zum Ersten erhielten nun auch die Armen einen Zugang zur medizinischen Versorgung in der Pestzeit, den sich zuvor vorrangig die vermögenden Bürger leisten konnten. Die große Anzahl der mittellosen Kranken wurde seither in den städtischen Brechhäusern auf Kosten der Stadt gepflegt. Zum Zweiten wurden spezielle Pestordnungen vom Rat erlassen, denen unmittelbar die Anleitungen der städtischen Ärzte zugrunde lagen. Dadurch wurden die Grundsätze der damaligen Pestlehre auch mit Hilfe der Ratsverordnungen innerhalb der Bevölkerung weiter getragen. 


\section{Quellen- und Literaturverzeichnis}

\section{Ungedruckte Quellen}

\section{Staats- und Stadtbibliothek Augsburg (SStBA)}

$2^{\circ}$ Cod. Aug. 53: Chronik der Stadt Augsburg bis 1635.

$2^{\circ}$ Cod. Aug. 71: Chronik der Stadt Augsburg von Math. Mannlich 911-1545.

$2^{\circ}$ Cod. Aug. 80: Anonymus, Die Elste Augspurger Cronica, hebt an von 302 nach Christi geburt, handelt von aufnemmen der Statt; und zeigt an, wie diese zum Christlichen glauben kommen, und was sy für anstöß gehabt hab. Bis 1596.

$2^{\circ}$ Cod. Aug. 214-215: Sammlung der wichtigsten Urkunden der Stadt Augsburg von Jo. Elias Leopold Herwart 1763, T. III: Supl. T. II; T. IV, Supl. T. III.

$2^{\circ}$ Cod. Aug. H 14: MaIR PAul HeKTOR, Ordnung und Beschreibung etlicher alter loeblicher Gebräuch und Herkommen, fürnehmlich wie es in den Reichstagen mit Empfahungen, Schankungen gehalten worden ist 1502-1579.

$2^{\circ}$ Cod. Aug. S 141: MAIR PAul HeKTOR, Memoribuch über alle Schanckungen, Gebräuche beim Ein- und Entgegenreiten hoher Personen...1501-1563.

$4^{\circ}$ Cod. Aug. 62: Rad JoH. MichaEl, Grabschriften auf den Gottesaeckern und in den Kirchen von Augsburg.

\section{Stadtarchiv Augsburg (StAA)}

Baumeisterrechnungen, 1494

Collegium Medicum:

IV: Doctores Medicinae in genere betr. (1460-1801 Fasc. 6)

Nr. 3: Pläne des Rates zur Anlegung des Gottesackers (1494)

Nr. 7: Schreiben der vier Augsburger Stadtärzte (1521)

Nr. 11: Schreiben der Stadtärzte vom 11. Juni 1532 und die darauf unternommenen Verordnungen des Rates (11. Juni 1532)

Nr. 13: Pestverordnungen des Rates $(1521,1534,1535,1547)$

Nr. 14: Verordnung gegen die unaprobierten Wundärzte (4. Juli 1535)

Nr. 24: Supplikation von Doktor Schludin an den Rat (aus dem Inhalt für 1535 datierbar) 
Ratsprotokolle: 1521, 1522, 1533, 1535, 1536, 1547, 1548.

Schätze:

Nr. 16: Eine Sammlung städtischer Verordnungen und Erlassen, angefertigt durch den Augsburger Stadtschreiber Johann Hagk 1536-1633

\section{Gedruckte Quellen}

AgRicola, Johann, Ain grüntlicher fleissiger außzug, auß allen bewerten Kriechischen und lateinischen lerern, dermassen bißher noch nye beschehen, Von ursachen, zaichen, fürsehung, und haylung der grewlichen Pestilentz sampt allen zufellen die sich in diser Kranckhait begeben mögen, Alles auß gutem grund, on all Sophistisch oder Arabisch, in der Artzney vngegründt, zusetz vnd erdichtes geschwetz. Durch Doct. Johan. Agricolam, der Artzney vnd Kriechischen sprach leser zu Jngolstat [Augsburg: Philipp Ulhart 10. März, 1533].

ANONYMUS, Ain regiment wider Die Pestilentz vast gut Vnd nutzlich $\mathrm{Zu}$ dyser zeyt [Augsburg: Johannes Schönsberger um 1525].

ANONYMus, Ain Pater noster zu beten, und zu Betrachten so unns Der Allmechtig got zu sendet Die pestilentz Krieg/ wider werigkait/ unser gwissen zu rainigen. Got unser sünd beklagen/ und in bitten sein zorn von uns wenden/ Dann auß ernstlich biten/ der berewten hertzen/ verhoffen wir/ got der allmechtig wurde lassen von seinem zorn/ und uns barmhertzigkait beweysen/ wie wol er uns strafft zu hail unser seel/ und auß unser sünd/ Aber er der in allem vil barmhertzig ist/ Rueffen wir an got unsern erloeser umb genad und barmertzigkait [Augsburg: Sigmund Grimm und Marx Wirsung 1521].

ARdEA, HANS, Ain gut und vast nutzliches Regiment wie man sich in der Pestilentz halten soll [Einbl. Augsburg: 1536], SStBA, 2 LR 204\#.

Augsburger Bevölkerungstafel Nr. 142 (1501-1679), 143 (1501-1744), 144 (1501-1771), 347 (1511-1584), SStBA, $2^{\circ}$ Lw Einblattdrucke nach 1500.

Augsburger gedruckte Pestordnung vom 12. August 1535, SStBA, $2^{\circ}$ S 14, Kapsel 1, Nr. 21.

BAIERLAND, ORTOLF VON, Versechung leib sele ere vnd gut [Augsburg: Hans Schobser 1490].

BiLD, Hieronymus (BeARB.), Der Ain vnnd neützig Psalm, Qui habitat in adiutorio altissimi Jst ein aertzney wider die Pestilentz Creütz vnd Leyden. Jn der weyß, O Herre Gott begnade mich [Augsburg: Heinrich Steiner 1544]. 
BÖHAM, MATTHIAS, Ain nutzliche/ vnnd für den gemainen Man/ genugsam gegründte vnderricht/ wie sich diser zeyt der Pestilentz halben zuhalten sey [Augsburg: gedruckt von Philipp Ulhart und zum Neudruck aufgelegt von Johannes Vogt dem Jüngeren um 1570; bearbeitet und ergänzt von Wolfgang Talhauser um 1547].

Christliche Erinnerung/ neben kurtzer historischer Verzaichnus/ Wie offt der Gott der Herr/ von Anno 1042 biß dahero/ wegen ubermachter Sünde/ mit der Pest/ neben anderen Plagen/ diese Statt Augsburg habe heimgesucht. Menigllich zu wahrer Buß und besserung des Leben (verfasst von Kaspar Augustin, Kupferstich von Raphael Custos, gedruckt in Augsburg 1629 von Johannes Schultes), in: WolfGang HaRMS (Hrsg.), Deutsche illustrierte Flugblätter des 16. und 17. Jahrhunderts, Bd. 1, Tübingen 1985, Nr. 236 :

Chroniken der deutschen Städte vom 14. bis 16. Jahrhundert, hrsg. von der Historischen Kommission bei der Bayerischen Akademie der Wissenschaften: Bd. 4 (Chronik von 1368-1406 mit Fortsetzung bis 1447; Chronik von der Gründung der Stadt Augsburg bis zum Jahre 1469; Chronik des Erhard Wahraus 1126-1445), Leipzig 1865; Bd. 5 (Chronik der Burghard Zink, 1368-1487), Leipzig 1866; Bd. 22 (Chronik des Hector Mülich, 13481487; Anonyme Chronik von 991-1483), Leipzig 1892; Bd. 23 (Chronik von Clemens Sender bis 1536), Leipzig 1894; Bd. 32 (Paul Hector Mairs I. Chronik von 1547-1565), Leipzig 1917.

Einblattdruck, Bild der Heiligen Sebastian und Rochus, [Augsburg oder Ulm: um 1460-1463], in: RichARD SCHMIDBAUER (Hrsg.), Ein unbekanntes Augsburger Pestblatt des 15. Jahrhunderts, in: Festschrift für Georg Leidinger zum 60. Geburtstag am 30. September 1930, München 1930, S. 229-231, Taf. 27.

Einblattdruck, Bild des strafenden Gottes (1) mit Christus und Maria, [Augsburg: um 14601470], in: Wilhelm Ludwig SchreIBER (Hrsg.), Handbuch der Holz- und Metallschnitte des XV. Jahrhunderts, 10 Bde. Stuttgart-Nendeln 1969, Taf. 751a.

Einblattdruck, Bild des strafenden Gottes (2) mit Christus und einem Heiligen, [Augsburg: um 1470-1480], in: WilHELM Ludwig SchreIBER (Hrsg.), Handbuch der Holz- und Metallschnitte des XV. Jahrhunderts, 10 Bde. Stuttgart-Nendeln 1969, Taf. 752.

Einblattdruck, Gebet zum Heiligen Sebastian mit seiner Bilddarstellung (1) [Augsburg, Hans Froschauer: um 1500], in: WILHELM LUDWIG SCHREIBER (Hrsg.), Handbuch der Holz- und Metallschnitte des XV. Jahrhunderts, 10 Bde. Stuttgart-Nendeln 1969, Taf. 1676b.

Einblattdruck, Gebet zum Heiligen Sebastian mit seiner Bilddarstellung (2) [vermutlich in Augsburg: um 1475], in: Wilhelm Ludwig SchreIBER (Hrsg.), Pestblätter des 15. Jahrhunderts, Straßburg 1918, Taf. 19. 
Einblattdruck, Gebete zu Heiligen Rochus und Sebastian mit ihren Bilddarstellungen [Augsburg: um 1473], in: Wilhelm Ludwig SchreIBER (Hrsg.), Handbuch der Holz- und Metallschnitte des XV. Jahrhunderts, 10 Bde. Stuttgart-Nendeln 1969, Taf. 1695c.

Einblattdruck, Wie sich der mensch halten sol wider die pestilentz. Und auch wie er sich regiren sol wenn sy ist. Und den rat sol man dick überlesen“, [Augsburg: Hermann Kästlin um 1483; Hans Schauer um 1495], in: KARL SudHOFF (Hrsg.), Ein neues deutsches Pestblatt, gedruckt zu Augsburg ca. 1483 bei Hermann Kästlin, in: Archiv für Geschichte der Medizin 3 (1910), S. 63-66; und in: WiLhELM Ludwig SChrEIBER (Hrsg.), Pestblätter des 15. Jahrhunderts, Straßburg 1918, Taf. 41.

ELLENBOG, UlRICH, Ain wunderbäre instruction und underwysung wider die pestilentz herfliessend von kayserlichem hoff und aller bewärtesten doctoribus in cristenlicher und haydescher nacion funden wärden mügen [Memmingen: Albrecht Kunnen 1494], ed. von ANTON BREHER, Der Memminger Stadtarzt Ulrich Ellenbog und seine Pestschriften, Berlin 1942, S. 39-89

DERS., Ordnung die doctor Ulrich von ellenbog Anno 1482 zuo Memingen der gemaind gesetzt hat, und ditz in der yetzigen anruor 1494 bestaett haut. di $\beta$ regiment und ordnung uff das kürtzest ist getailt in zwai capittel [Memmingen: Albrecht Kunnen 1494], ed. von ANTON BREHER, Der Memminger Stadtarzt Ulrich Ellenbog und seine Pestschriften, Berlin 1942, S. 90-98.

Engel, Johann, Tractat von der Pestilentz Joanni Engel, der freyen künsten und artzney Doctor/ au $\beta$ der leer der Doctorn der artzney und Astronomey gezogen [Augsburg: Sigmund Grimm und Marx Wirsung 4. November 1518].

FENCKEN, MichAEL, Ain gut nützlich Regiment, wie man sich halten soll, vor, in, vnd nach der zeyt der erschröcklichen kranckhayt der Pestilentz/ durch Michaelem Fencken, der Artzney Doctorem, diser zeyt Ordinarien der löblichen Vniuersitet zu Ingolstat [Augsburg: Alexander Weißenhorn 1533].

Folz, HANS, Spruch von der Pestilentz, ed. von ERnSt MARTIN, Strassburg 1879, S. 1-18.

GASSER, ACHILles PIRMIN, Ander und Dritter Theil/ Der weitberümpten Keyserlichen Freyen vnd deß H. Reichßstatt Augspurg in Schwaben/ Chronica / Auß Achillis P. Gasseri ... Chronica ... gezogen/ vnnd ... inn vnser Teutsche Spraach inn offentlichen Truck gegeben/ ... Durch Wolffgangum Hartmannum Pfarherrn zu Eberspach, Getruckt zu Franckfurt am Mayn/ bey Christ. Egen. Erben, 1595-1596. 
Hagenmeyer, Christa, Die 'Ordnung der Gesundheit' für Rudolf von Hohenberg. Untersuchungen zur diätetischen Fachprosa des Spätmittelalters mit kritischer Textausgabe. Phil. Diss. Heidelberg 1972.

JACOBI, JoHANNES, Tractatus de pestilentia [Augsburg: Johann Keller um 1480].

JunG, AmBrosius, Ein außerwelt loblich tractat/ und regiment in dem schwaeren zeit der pestilentz au $\beta$ / gezogen. au $\beta$ den bewaertin und weysisten alten gsch/ rifften der artzney. Durch Ambrosium jung der siben/ freyen kunst und der artzney doctor. der zeit wirdigen/ herrn vom thum zu Augspurg geschworner doctor [Augsburg: Hans Schönsberger, 14. November 1494].

DERS., Tractatulus perutilis de pestilentia [Augsburg: Hans Schönsberger, 20. November 1494].

DERS., Ain nutzliche trostliche und kurze underrichtung (1)/ wie man sich in disen schwären leüffen der pestilentz halten sol / durch Doctor Ambrosium Jungen / statarzt zu Augspurg / dem gemainen mann zu hilff und guettem / der nit andern beystand hat / verordnet [Augsburg: Silvan Ottmar 1521].

DERS., Ain nutzliche trostliche und kurze underrichtung (2)/ wie man sich in disen schwären leüffen der pestilentz halten sol / dem gemainen mann zu hilff und guettem / der nit andern beystand hat/ verordnet durch Doctor Ambrosium Jungen / den ältesten und erfarnsten Artzet, zu Augspurg [Augsburg: Silvan Ottmar 1535]

DERS., Ain nutzliche trostliche und kurze underrichtung (3)/ wie man sich in disen schwären leüffen der pestilentz halten sol / durch Doctor Ambrosium Jungen / der Artzney Doctor zu Augspurg / dem gemainen mann zu hilff und guettem / der nit andern beystand hat / verordnet [Augsburg: Silvan Ottmar 1539, Valentin Ottmar 1563; Strassburg: Wendelin d. Ä. Rihel 1535; Innsbruck: Rupprecht Höller 1562].

Jung, Ambrosius/ Occo, Adolph II./ TiefFenbach, Johann/ Sailer, Gereon/ Behem, DAMIAN/ Schludi, BERnHARD/ VoGT, JOHANN, Conclusiones et propositiones universam Medicinam, per genera comprehendentes, ad eum modum quem Augustana Medici iuxta placita veterum medicorum observant, apertis ulnis amplecentur et circa aegrotos curationes instituunt [Augsburg: Philipp d. Ä. Ulhart 1538].

KLAINMÜLLER (WOHLOPTER), JOHANN, Ain hymlischen vnn Natürlichen anzaygung dieser sterberlichen leüff mit ainem nutzlichen vnd trostlichen regiment wie sich der Mensch hallten soll mit aderlassen Ertzneyen vnn guotter regierung zuo Eren ainer gantzer gemain der kayserlichen Stat Augspurg geordnet durch Doctorem Johannem Klainmüller oder Waelhopter [Augsburg: Hans Schönsberger, 12. Oktober 1521]. 
Lobera de Avila, Luis/ Krautwadel, Michael (Bearb.), Ein nutzlich Regiment der gesundtheyt/ Genant das Vanquete/ oder Gastmal der Edlen Diener von der Complexion/ Eigenschafft/ Schad/ und Nutz allerley Speyß/ Trancks/ und von allem/ darmit sich der Mensch in Gesundtheyt enthelt/ Mit sampt einem kurtzen Regiment/ Wye man sich in der Pestilentz/ Pestilentzischen Fieber und Schweyß halten soll [Augsburg: Heinrich Steiner $1531]$.

LOBERA DE AviLA, LuIS, Vanquete de nobles cavalleros e modo de bivir desole que se leuantan hasta q se acuestan, y habla de cada manjar que complexion y propiedad tienee que danos y prouechos haze, e tra ta del regimento curativo e preservativo delas fiebres Pestilencia e otrus cosas utillisimas [Augsburg: Heinrich Steiner 1530].

Luther, Martin, Ob man vor dem sterben fliehen möge, Wittenberg 1527, in: D. Martin Luthers Werke: Kritische Gesamtausgabe. Schriften, Bd. 23, Weimar 1901, S. 323-379.

Magenbuch, JoHAnnes, Ain nützlichs vnd Bewaerts gemains Regiment/ vnd für kommen des vergyfften luffts/ in der zeyt des Sterbens oder Regierung der Pestilentz/ Durch den Hochgeleerten Doctor Johann Magenbuch von Nuernberg/ menigklich one schaden zu brauchen [Augsburg: Philipp Ulhart d. Ä. um 1530].

MEYer, Christian (Hrsg.), Die Hauschronik der Familie Holl (1487-1646) insbesondere die Lebensaufzeichnungen des Elias Holl, Baumeisters der Stadt Augsburg, München 1910.

Milchtaler, Johannes, Ain Kurtz vnd notwendig vnderricht vnd Regiment/ wie sich zu zeyten der Pestilentz/ vorhin vnd so ainer mit/ oder ohn außwendige zaychen des leybs antast wurde/ auffs fürderlichst halten vnnd artzneyen solle/ Durch Doctor Johansen Milchtaler/ Leybartzet zů Schwatz [Augsburg: Heinrich Steiner 1534].

Musculus, Wolfgang (Bearb.), Der ain vnd neintzichst Psalm tröstlich in der Gemain zu der Zeyt der Pestilentz zu singen [Augsburg: um 1540].

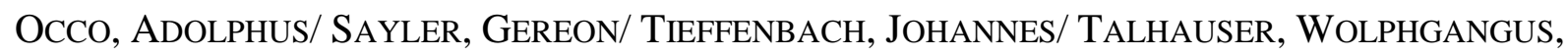
Was die Pestilentz an jr selbs sey/ mit jren ursachen und Ertzneyen/ Den Ersamen und Weysen Bürgermaister und Rathe/ auch gantzer gemain der statt Augspurg/ durch die bestelten doctores daselbst/ zu nutz und gut gemacht und beschriben [Augsburg: Philipp Ulhart 1535].

Ordnung der Gesundheit, ed. von CHRISTA HAGENMEYER, Die 'Ordnung der Gesundheit' für Rudolf von Hohenberg. Untersuchungen zur diätetischen Fachprosa des Spätmittelalters mit kritischer Textausgabe. Phil. Diss. Heidelberg 1972, S. 275-450.

OSIANDER, ANDrEAS, Wie und wohin ein Christ fliehen soll, Nürnberg, 1533, ed. von BERNHARD SCHNEIDER, in: Osiander, Andreas d. Ä.: Gesamtausgabe, hrsg. von GERHARD 
MüLler/ GotTFRIED SEeBAß, Bd. 5. Schriften und Briefe 1533 bis 1534, Gütersloh 1983 S, 384-411.

PARACELSUS, Der grossen Wundartzney/ das Erst (ander) Buch/ des Ergründeten und bewerten/ der bayden artzney/ Doctors Paracelsi/ von allen wunden/ stich/schüß/ braend/ thierbiß/ baynbrüch/ und alles was die wundartzney begreifft. Von der alten und neüwen künsten erfyndung/ nichts underlassen [Augsburg: Heinrich Steiner 1536].

Petrarca, Francesco/ Vigilius, StePhanus (Übers.), Das Glückbuch/ Beydes des Gutten und Boesen/ darin leere und trost/ wesz sich menigklich hierin halten sol [Augsburg: Heinrich Steiner 1539].

Seitz, AleXANDER, Ain schoener Tractat von dem Saturnischen gschoß der Pestilentz mit ettlichen klugen fragstucken/ darinn der ungelert man sich irret/ zu gferlichem abbruch seins lebens/ ee zeit/ alles durch den hochgelerten philosophum Alexander Seitzen von Marckbach Doctor zu München/ auss menschlicher lieb trewlich beschriben/ und verantwurt [Augsburg: Otmar Silvan, 15. Februar 1522], in: Peter UkenA (Hrsg.), Alexander Seitz sämtliche Schriften, Bd. 1: Medizinische Schriften, Berlin 1970, S. 143 207.

DERS., Dem fursichtigen Ersamen und weysen Burger maister und Ratte/ der loblichen Statt München/ ain schoener nützlicher tractat von aderlassen/ durch den hochgelerten Doctor Alexander Sytz vonn Marpach vleyssigclich beschriben [Landshut: Johann Weyssenburger 1520].

SteINHÖWEL, HEINRICH, Büchlin der ordnung/ wie sich der mensch halten sol/ zu den zyten dieser grusenlichen kranckheit [Ulm: Johannes Zainer von Reutlingen, 11. Januar 1473], ed. von ARNOLD C. KLEBS/ KARL SudHOFF, Die ersten gedruckten Pestschriften, München 1926.

von Stetten, Paul, Geschichte der Heil. Röm. Reichs Freyen Stadt Augsburg/ Aus Bewährten Jahr=Büchern und Tüchtigen Urkunden gezogen/ und an das Licht gegeben, 2 Bde. Frankfurt/Leipzig 1743/1758.

Stocker, Johannes, Ain Regiment für die Pestilentz/ Durch den hochgelerten Johan Stockar/ Doctor der Ertzney/ vnd Stat Artzt zu Vlm beschriben vnd in seynen leben gebraucht vnd geyebt [Augsburg: Sigmund Grimm/Marx Wirsung 1520].

TheophiLus, Johannes, Zwu Gotselige/ Gnadenreiche vnd Christliche/ Bekantnussen unsers heyligen Christlichen Glaubens/ Darmit ein yeder Christ/ so selig begert zu werden/ zu yeder zeyt seines lebens gewapnet und geruest sein sol/ Vorab in disen fehrlichen leuefften und unfall der grausamen Pestilentz/ So uber alle ander Artzney ganz nuetzlich/ 
dienstlich und troestlich sein/ zu disem Newen Jahre (...) [Augsburg: Heinrich Steiner $1544]$.

TORMANITA, HANS (später genannt als HANS ARDEA), Vil menschen weren der pestilentz frey Weyßten sy dar für ein rechte ertzney (1) [Einbl. Augsburg: Günther Zainer um 1472], in: WilHelm Ludwig SchreIBER (Hrsg.), Pestblätter des 15. Jahrhunderts, Straßburg 1918, Taf. 20.

TORMANITA, HANS (später genannt als HANS ARDEA), Vil menschen weren der pestilentz frey Weyßten sy dar für ein rechte ertzney (2) [Einbl. Augsburg: Günther Zainer um 1472], ed. von KARL SUdHOFF, in: Archiv für Geschichte der Medizin, Bd. 2, Leipzig 1909, S. 113.

ÜBERLINGEN, ANDREAS vON, Pestilentzbůchlin/ das da antzaigt Wie vnd wamit man sich vor der Pestilentz bewaren soll Vnd ob ain mensch damit vergifft wurd/ wie vnd mit was artzney er jm helffen mag. Gemacht von dem Hochgelerten Maister Andres von Vberlingen der artzney beruempten Doctor. Jtem ain ander regimant/ durch maister Andres Reichlin/ auch Doctor der artzney/ gar maisterlich gesetzt [Augsburg Johann Otmar, 27. Oktober 1512].

VERINGER, MARCUS DEAS, Ein kurtz nützlichs Regiment/ wie sich ein jegklicher vor der Pestilentz bewaren/ und der selben entpfliehen/ auch mit hilff des Allmechtigen Gottes/ seines leibs gesundhait/ erhalten soll/ Darzu den krancken mit dieser sucht der Pestilentz beladen/ fast dienlich. Marcus Deas Veringer der Artzney Doktor/ und Stadt Artzet zum Newenmarckt [Augsburg: Heinrich Steiner 1533].

Vogter, BARTHOlOMEUs, Ajn nutzlich vnnd notwendigs Artzney Buechlin für den gemainen menschen Darinnen von allen Kranckhaiten allerlay art so dem menschen zuestehn moegen die zuo vertreiben/ Mit vil bewerten stucken Kreütern/ Salben/ Pflaster/ und Recepten u. Durch den weytberuembten Maister Bartholome Vogter/ Augen Artzt zu Dillingen / Bey dem Hochwirdigen u. Herrn/ Herren Cristoff Bischoffen zu Augspurg/ newlich beschriben und inn Truck gegeben [Augsburg: Philipp Ulhart 1531].

WOLFHART, UlRICH, Ain kurtzer Bericht von Doctor Ulrich Wolfhart zu Memmingen, wie man sich vor der erschröcklichen kranckhait des gebrechens der Pestilentz preserviern und bewaren mög/ Auch wann ains darmit angriffen/ wie es sich halten/ vnd die bewaerten kostlichen stuck/ darzu dienstlich/so er auf das best in seiner Apoteck/ so die Alt genennt wirdt/ har lassen zurichten/ gebrauchen/ Got zu lob vnd ehr/ gmainer stat Memmingen/ auch maenigklich zu nutz vnd wolfart [Augsburg: Philipp Ulhart um1535].

ZWICKER, ADAM, Ein kurtze wolgegründte underrichtung und erklerung von Doctor Adam Zwicker zu Memmingen, wie man sich vor der erschröcklichen kranckheit der Pestilentz 
praeservieren/ unnd bewaren soll/ und wenn eins darmit angriffen würde/ wie es sich halten soll/ mit bewerten köstlichen stücken brauchen Gott dem herren zu lob und ehr/ zu nutz gemeiner löblichen statt zu Memmingen [Augsburg: Silvan Ottmar 1537].

\section{Literatur}

BÁTORI, IngRID, Die Reichsstadt Augsburg im 18. Jahrhundert. Verfassung, Finanzen und Reformversuche (=Veröffentlichungen des Max-Planck-Instituts für Geschichte, Bd. 22), Göttingen 1969.

Benedictow, Ole J., Plague in the Late Medieval Nordic Countries. Epidemiological Studies. Oslo 1992.

Bergdolt, Klaus, Der Schwarze Tod in Europa. Die große Pest und das Ende des Mittelalters, München 1994.

DERS., Die Pest. Geschichte des Schwarzen Todes, München 2006.

BIRABEN, JEAN-NoËL, Les hommes et la pest en France et dans les pays européens et mediterranéens, 2 Bde., Paris/Den Haag 1975/76.

BISLE, MAX, Die öffentliche Armenpflege der Reichsstadt Augsburg mit Berücksichtigung der einschlägigen Verhältnisse in anderen Reichsstädten Süddeutschlands, Paderborn 1904.

Blankenburg, Susann, Das Hebammenwesen Augsburgs zur Zeit der Reichsstadt: unter besonderer Berücksichtigung der Hebammenordnungen, Diss. Univ. Ulm 2003.

BRANDIS, TILO, Handschriften- und Buchproduktion im 15. und frühen 16. Jahrhundert, in: LUdGER GRENZMANN/ KARL StACKMANN (Hrsg.), Literatur und Laienbildung im Spätmittelalter und in der Reformationszeit. Symposion Wolfenbüttel (Germanistische Symposien. Berichtsbände 5), Stuttgart 1981, S. 176-196.

BRAUN, PlaCIDUS, Geschichte der Bischöfe von Augsburg: chronologisch und diplomatisch verfasst, und mit historischen Bemerkungen beleuchtet, 4 Bde., Augsburg 1814-1815.

Breher, Anton, Der Memminger Stadtarzt Ulrich Ellenbog und seine Pestschriften, Berlin 1942.

Bulst, NeITHARD, Heiligenverehrung in Pestzeiten. Soziale und religiöse Reaktionen auf die spätmittelalterlichen Pestepidemien, in: ANDREA LÖTHER u.a. (Hrsg.), Mundus in imagine. Bildersprache und Lebenswelten im Mittelalter. Festgabe für Klaus Schreiner mit einem Geleitwort von Reinhart Kosseleck, München 1996, S. 63-97. 
DERS., Krankheit und Gesellschaft in der Vormoderne: das Beispiel der Pest, in: NeITHARD Bulst/ Robert Delort (Hrsg.), Maladies et société (XIIe-XVIII siècle), Paris 1989, S. $17-47$.

DERS., Die Pest verstehen: Wahrnehmungen, Deutungen und Reaktionen im Mittelater und in der Frühen Neuzeit, in: Dieter Groh/ Michael Kempe/ Franz Mauelshagen (Hrsg.), Naturkatastrophen. Beiträge zu ihrer Deutung, Wahrnehmung und Darstellung in Text und Bild von der Antike bis ins 20. Jahrhundert, Tübingen 2003, S. 145-163.

DERS., Der Schwarze Tod. Demographische, wirtschafts- und kulturgeschichtliche Aspekte der Pestkatastrophe von 1347-1352. Bilanz der neueren Forschung, in: Saeculum 30, 1979, S. 45-67.

Bulst, Neithard/ Delort, Robert (Hrsg.): Maladies et société (XIIe-XVIII siècle), Paris 1989.

Bulst, Neithard/ Spieß, KARL-Heinz (Hrsg.), Sozialgeschichte mittelalterlicher Hospitäler, (Vorträge und Forschungen, Konstanzer Arbeitskreis für Mittelalterliche Geschichte. Band 65), Ostfildern: Thorbecke, 2007.

BüHL, Charlotte, Die Pestepidemien des ausgehenden Mittelalters und der frühen Neuzeit in Nürnberg (1483-1533). Zulassungsarbeit Erlangen. Wieder in: RUDOLF ENDRES (Hrsg.), Nürnberg und Bern. Zwei Reichsstädte und ihre Landgebiete, Erlangen 1990, S. 121-168.

BÜHLER, HANS VIKTOR: Das Ärztegeschlecht der Occo, in: Suddhoffs Archiv für Geschichte der Medizin und Naturwissenschaften 28 (1935), S. 14-42.

BurCKHARD, GEORG, Ein bis jetzt unbekannter medizinischer Schriftsteller des 16. Jahrhunderts (Dr. Johannes Michael aus Schwaz), Sudhoffs Archiv 4 (1911), S. 20-30.

CAmpbell, A. Montgomery, The Black Death and Men of Learning, New York 1931.

Carmichael, Ann G., Plague and the poor in Renaissance Florence, Cambridge 1986.

Cipolla ,CARlo M., Public Health and the Medical Profession in the Renaissance, Cambridge 1976.

Clasen, Claus Peter, Arm und Reich in Augsburg vor dem Dreißigjährigen Krieg, in: GunTHeR GotTLIEB u.a. (Hrsg.), Geschichte der Stadt Augsburg von der Römerzeit bis in die Gegenwart. Stuttgart 1985, S. 312-336.

Ders., Armenfürsorge (1) in Augsburg, in: GunTHER GotTlieb u.a. (Hrsg.), Geschichte der Stadt Augsburg von der Römerzeit bis in die Gegenwart. Stuttgart 1985, S. 337-342.

DERS., Armenfürsorge (2) in Augsburg vor dem Dreißigjährigen Krieg, in: Zeitschrift des Historischen Vereins für Schwaben 78 (1984), S. 65-115. 
Cohn, SAmuel K., The Black Death and the Burning of Jews, in: Past and Present, No. 196 (August 2007), S. 3-36.

DERS., The Black Death Transformed. Disease and Culture in Early Renaissance Europe, London-Oxford-New York 2002.

Cunningham, Andrew, Transforming plague. The laboratory and the identity of infectious disease, in: ANDREw Cunningham/ PERry WiLliams (ed.), The laboratory revolution in medicine, Cambridge 1992, S. 209-244.

DIEMINGER, WERnER, Bader, Barbiere, Wund- und Zahnärzte in der Reichsstadt Augsburg von 1316 bis 1806, Diss. Univ. Ulm 1999.

DIENINGER, FrIEDRICH, Das Collegium Medicum Augustanum, in: Deutsches Ärzteblatt 61 (1964), S. 1239-1243.

Dieudonné, Adolf, Die Pest in den letzten Jahrhunderten, Leipzig 1906.

Dinges, Martin, Neue Wege in der Seuchengeschichte?, in: MARTin Dinges/ ThOMAS ScHLICH (Hrsg.), Neue Wege in der Seuchengeschichte (Medizin, Gesellschaft und Geschichte. Jahrbuch des Instituts für Geschichte der Medizin der Robert Bosch Stiftung, Beiheft 6), Stuttgart 1995, S. 7-24.

DerS., Pest und Pestpolitik in der europäischen Neuzeit, in: Mischa MeIER (Hrsg.), Pest. Die Geschichte eines Menschheitstraumas, Stuttgart 2005, S. 283-313, 440-450.

DERS., Pest und Staat: Von der Institutionengeschichte zur sozialen Konstruktion?, in: Martin Dinges/ Thomas Schlich (Hrsg.): Neue Wege in der Seuchengeschichte (Medizin, Gesellschaft und Geschichte. Jahrbuch des Instituts für Geschichte der Medizin der Robert Bosch Stiftung, Beiheft 6), Stuttgart 1995, S. 71-97.

Ders., Seuchen in Mittelalter und Früher Neuzeit, in: Petra FeuersteIn-Herz (Hrsg.), Gotts verhengnis und seine straffe - zur Geschichte der Seuchen in der Frühen Neuzeit, (Ausstellungskataloge der Herzog August Bibliothek Nr. 84), Braunschweig 2005, S. 1526.

DERS., Süd-Nord-Gefälle in der Pestbekämpfung. Italien, Deutschland und England im Vergleich, in: WOLFGANG U.EcKART/ ROBERT JÜTTE (Hrsg.): Das Europäische Gesundheitssystem, Stuttgart 1994, S. 19-51.

Dinges, Martin/ Schlich, ThOMAs (Hrsg.), Neue Wege in der Seuchengeschichte (Medizin. Gesellschaft und Geschichte. Jahrbuch des Instituts für Geschichte der Medizin der Robert Bosch Stiftung, Beiheft 6), Stuttgart 1995.

DORMEIER, HEINRICH, "Ein geystliche ertzney fur die grausam erschrecklich pestilentz". Schutzpatrone und frommer Abwehrzauber gegen die Pest, in: Das große Sterben. 
Seuchen machen Geschichte, hrsg. von Hans WiLDEROTTER. Ausst. Kat. Dresden 1996, S. 54-93.

DERS., Laienfrömmigkeit in den Pestzeiten des 15./16. Jahrhunderts, in: NeITHARD BulST/ Robert Delort (Hrsg.): Maladies et société (XIIe-XVIII siècle), Paris 1989, S. 269-306.

Drancourt, Michel/ RAOUlt, DiDIER, Molecular insights into the history of plague, in: Microbes and Infection 4 (2002), S. 105-109.

Du CANGe, Charles du Fresne, Glossarium mediae et infimae Latinitatis, Graz 1883-1887.

ECKER-OFFENHÄUßER, UTE, "Pest, Franzosen, Scharbock" - Krankheitserfahrung und medizinischer Alltag des 17. Jahrhunderts im Spiegel der Werke des Augsburger Wundarztes Joseph Schmid. Magisterarbeit, Augsburg 1994.

DIES., Joseph Schmid. Handwerkschirurg und Schriftsteller in Augsburg im 17. Jahrhundert, in: Medizin, Gesellschaft und Geschichte (15) 1997, S. 117-139.

EsSER, THILO, Pest, Heilsangst und Frömmigkeit. Studien zur religiösen Bewältigung der Pest am Ausgang des Mittelalters (Münsteraner Theologische Abhandlungen 58), Altenberge 1999.

Feldmann, Dominik/ Walser, Andrea/ Landois, Antonia/ Pfotenhauer, Bettina (Hrsg.), Der Feind in der Stadt: vom Umgang mit Seuchen in Augsburg, München und Nürnberg. Eine Ausstellung der Bayerischen Archivschule der Generaldirektion der Staatlichen Archive Bayerns, München 2016.

Feuerstein-Herz, Petra (Hrsg.), Gotts verhengnis und seine straffe - zur Geschichte der Seuchen in der Frühen Neuzeit, (Ausstellungskataloge der Herzog August Bibliothek Nr. 84), Braunschweig 2005.

DIES., Im Druck der Seuchen - Seuchen und Buchdruck in der Frühen Neuzeit am Beispiel der Bestände der Herzog August Bibliothek, in: DIES. (Hrsg.), Gotts verhengnis und seine straffe - zur Geschichte der Seuchen in der Frühen Neuzeit, (Ausstellungskataloge der Herzog August Bibliothek Nr. 84), Braunschweig 2005, S. 27-36.

FISCHER, ALFONS, Geschichte des deutschen Gesundheitswesens, Bd. 1: Vom Gesundheitswesen der alten Deutschen zur Zeit ihres Anschlusses an die Weltkultur bis zum Preußischen Medizinaledikt (Die ersten 17 Jahrhunderte unserer Zeitrechnung), Berlin 1933.

FISCHER, HERMANN (Hrsg.), Schwäbisches Wörterbuch, Tübingen 1904-1936.

FISCHER, THOMAS, Städtische Armut und Armenfürsorge im 15. und 16. Jahrhundert. Sozialgeschichtliche Untersuchungen am Beispiel der Städte Basel, Freiburg i. Br. und 
Straßburg (Göttinger Beiträge zur Wirtschafts- und Sozialgeschichte, Bd. 4), Göttingen 1979.

FleischmanN, JOSEF, Die Ärztefamilie Jung, in: Lebensbilder aus dem bayerischen Schwaben Bd. 4, München 1955, S. 14-25.

Fossel, ViKTOR, Hieronymus Fracastoro: Drei Bücher von den Kontagien, den kontagiösen Krankheiten und deren Behandlung (1546), Leipzig 1910.

FÖßEL, Amalie, Der schwarze Tod in Franken 1348-1350, Magisterarbeit Erlangen 1986. Wieder in: Mitteilungen des Vereins für Geschichte der Stadt Nürnberg 74 (1987), S. 177.

FrIES, KARL, Pest zu Augsburg im dreißigjährigen Krieg. Ein Beitrag zur Geschichte der Pest. Diss. Med. Augsburg 1887.

GeFfCKen, Peter, Münze(n), in: Günther Grünsteudel/ Günter Hagele/ Rudolf FrANKENBERgER (Hrsg.), Augsburger Stadtlexikon, Augsburg 1998, S. 667-670.

DERS., Soziale Schichtung in Augsburg 1396 bis 1521. Beitrag zu einer Strukturanalyse Augsburgs im Spätmittelalter, Diss. München 1983.

Gensthaler, Gerhard, Das Medizinalwesen der Freien Reichsstadt Augsburg bis zum 16. Jahrhundert: mit Berücksichtigung der ersten Pharmakopöe von 1564 und ihrer weiteren Ausgaben, Augsburg 1973.

GEORGES, KARL ERNST, Ausführliches lateinisch-deutsches Handwörterbuch: aus den Quellen zusammengetragen und mit besonderer Bezugnahme auf Synonymik und Antiquitäten unter Berücksichtigung der besten Hilfsmittel, Hannover 1916-1919.

Gier, Helmut/ Janota, Johannes (Hrsg.), Augsburger Buchdruck und Verlagswesen: von den Anfängen bis zur Gegenwart, Wiesbaden 1997.

Gorbach, Sherwood L./ Bartlett, John G./ Blacklow, NeIl R. (Hrsg.), Infectious Diseases, $2^{\text {nd }}$ Edition, Philadelphia 1998.

GotTlieb, Gunther u.a. (Hrsg.), Geschichte der Stadt Augsburg von der Römerzeit bis in die Gegenwart, Stuttgart 1985.

Graus, FrantiseK, Pest, Geißler, Judenmorde. Das 14. Jahrhundert als Krisenzeit (Veröffentlichungen des Max-Planck-Instituts für Geschichte 86), Göttingen 1987.

GRIMM, JACOB UND WILHELM, Deutsches Wörterbuch, 16. Bde., Leipzig 1854-1960.

GRMEK, MIRKO DRAŽEN, Diseases in the Ancient Greek World, Baltimore/London 1989.

Groß, UwE, Kurzlehrbuch Medizinische Mikrobiologie und Infektiologie, 2. Auflage, Stuttgart 2009. 
GrÜNSTEUdel, GÜNTHER/ HAGELE, GÜNTER/ FrAnKEnBERger, RudOlF (Hrsg.), Augsburger Stadtlexikon, Augsburg 1998.

HeCKER, Justus FrIEDRICH KARL, Der Schwarze Tod im vierzehnten Jahrhundert. Nach den Quellen für Aerzte und gebildete Nichtärzte bearbeitet, Berlin 1832.

DERS., The Black Death in the Fourteenth Century, London 1833; New York 1835, 1859 und 1885.

HAESER, HEINRICH, Lehrbuch der Geschichte der Medizin und der epidemischen Krankheiten, 3 Bde., Jena 1882.

Hahn, Helmut/ Kaufmann, Stefan H. E./ Schulz, Thomas F./ Suerbaum, Sebastian (Hrsg.), Medizinische Mikrobiologie und Infektiologie, 6. komplett überarb. Auflage, Heidelberg 2009.

Hammond, Mitchell, The Origins of Civic Health Care in Early Modern Germany, Diss. Phil., Blacksburg Virginia 2000.

Hatje, Frank, Leben und Sterben im Zeitalter der Pest. Basel im 15. bis 17. Jahrhundert, Basel-Frankfurt/M. 1992.

HECKER, Justus FRIEDRICH KARL, Die großen Volkskrankheiten des Mittelalters. Historischpathologische Untersuchungen, Berlin 1865.

HeINDL-HeInZ, IDAmARIE, Das Gesundheitswesen der Stadt Augsburg im 17. und 18. Jahrhundert, München 1950.

Henderson, JoHn, Epidemics in Renaissance Florence: Medical Theory and Government Response, in: NeITHARd Bulst/ Robert Delort (Hrsg.), Maladies et société (XIIeXVIII siècle), Paris 1989, S. 165-186.

Herberger, TheOdor, Die Seelhäuser und die Seelgeräthe in Augsburg, in: Zeitschrift des Historischen Vereins für Schwaben und Neuburg 3 (1876), S. 283-296.

DERS., Die St. Jakobs-Pfründe in Augsburg, Augsburg 1848.

HERLIHY, DAVID, Der Schwarze Tod und die Verwandlung Europas, Berlin 1997.

HoENIGER, RoBert, Der Schwarze Tod in Deutschland, Berlin 1882.

HOFFMANN, RoBERT, Die Augsburger Bäder und das Handwerk der Bader, in: Zeitschrift des Historischen Vereins für Schwaben und Neuburg 12 (1885), S. 1-33.

HöRmANN, LuDwIG, Zur Geschichte des Heilig-Geist-Hospitals in Augsburg, in: Zeitschrift des Historischen Vereins für Schwaben und Neuburg 6 (1879), S. 145-176.

HofFMANN, RoBert, Die Augsburger Bäder und das Handwerk der Bader, in: Zeitschrift des Historischen Vereins für Schwaben und Neuburg 12 (1885), S. 1-33. 
HÖFLER, MAX, Deutsches Krankheitsnamen-Buch, München 1899 [Nachdruck: Hildesheim 1970].

Horrox, Rosemary, The Black Death, Manchester 1994.

HöHL, MONIKA, Die Pest in Hildesheim: Krankheit als Krisenfaktor im städtischen Leben des Mittelalters und der Frühen Neuzeit (1350-1750), Hildesheim 2002.

IBS, JÜRgEN H., Die Pest in Schleswig-Holstein von 1350 bis 1547/48. Eine sozialgeschichtliche Studie über eine wiederkehrende Katastrophe, Frankfurt a.M. u.a. 1994.

IMMENKRÖTER, HERBERT, Kirche zwischen Reformation und Parität, in: GUNTHER GOTTLIEB u.a. (Hrsg.), Geschichte der Stadt Augsburg von der Römerzeit bis in die Gegenwart. Stuttgart 1985, S. 391-412.

JACQUART, DANIELle, La médecine médiévale dans le cadre parisien: XIVe - XVe siècle, Paris 1998.

JAHN, JOACHIM, Die Augsburger Sozialstruktur im 15. Jahrhundert, in: GunTHER GOTTLIEB u.a. (Hrsg.), Geschichte der Stadt Augsburg von der Römerzeit bis in die Gegenwart. Stuttgart 1985, S. 187-193.

DERS., Augsburgs Einwohnerzahl im 16. Jahrhundert - ein statistischer Versuch, in: Zeitschrift für Bayerische Landesgeschichte 39 (1976), S. 379-396.

JANKRIFT, KAY PETER, Up dat god sich aver uns verbarmen wolde. Formen, Strukturen und Entwicklungen der Auseinandersetzung mit Seuchen in westfälischen und rheinischen Städten im Mittelalter (8.-16. Jh.) [Habilitationsschrift, Münster 2002].

JÄCKEL, DIRK, Judenmord-Geißler-Pest: Das Beispiel Straßburg 1349, in: MischA MEIER (Hrsg.), Pest. Die Geschichte eines Menschheitstraumas, Stuttgart 2005, S. 162-178.

JENTSCH, ALEXANDER, Regimen sanitatis von Heinrich von Loufenberg ein mittelhochdeutsches Gedicht untersucht und erläutert, Diss. Phil. Strassburg 1908.

JoACHIMSOHN, PAUL, Zur städtischen und klösterlichen Geschichtsschreibung Augsburgs im fünfzehnten Jahrhundert, in Allemania, Bd. XXII (1894), S. 1-32, 123-159.

JÜTTE, RoBert, Arme, Bettler, Beutelschneider: eine Sozialgeschichte der Armut in der Frühen Neuzeit, Weimar 2000.

DERS., Ärzte, Heiler und Patienten: medizinischer Alltag in der frühen Neuzeit, München 1991.

DERS., Obrigkeitliche Armenfürsorge in deutschen Reichsstädten der Frühen Neuzeit. Städtisches Armenwesen in Frankfurt am Main und Köln, Köln 1984. 
KellenbenZ, HermanN, Wirtschaftsleben der Blütezeit, in: GunTHER GotTlieb u.a. (Hrsg.), Geschichte der Stadt Augsburg von der Römerzeit bis in die Gegenwart. Stuttgart 1985, S. 258-301.

KIEßLING, RoLF, Bürgerliche Gesellschaft und Kirche in Augsburg im 14. und 15. Jahrhundert. Ein Beitrag zur Strukturanalyse der spätmittelalterlichen Stadt, Augsburg 1971.

Ders., Bürgertum und Kirche im Spätmittelalter, in: GunTHER GotTlieb u.a. (Hrsg.), Geschichte der Stadt Augsburg von der Römerzeit bis in die Gegenwart. Stuttgart 1985, S. 208-213.

Ders., Augsburg zwischen Mittelalter und Neuzeit, in: GunTHER GotTlieb u.a. (Hrsg.), Geschichte der Stadt Augsburg von der Römerzeit bis in die Gegenwart. Stuttgart 1985, S. 241-251.

Ders., Augsburgs Wirtschaft im 14. und 15. Jahrhundert, in: GunTHER GotTLIEB u.a. (Hrsg.), Geschichte der Stadt Augsburg von der Römerzeit bis in die Gegenwart. Stuttgart 1985, S. 171-181.

KinZelbach, ANNEMARIE, Gesundbleiben, Krankwerden, Armsein in der frühneuzeitlichen Gesellschaft. Gesund und Kranke in den Reichsstädtischen Überlingen und Ulm, 15001700 (Medizin, Gesellschaft und Geschichte. Jahrbuch des Instituts für Geschichte der Medizin der Robert Bosch Stiftung, Beiheft 8), Stuttgart 1995.

KLEBS, ARNOLD, Geschichtliche und bibliographische Untersuchungen, in: DeRS./ KARL SudHOFF, Die ersten gedruckten Pestschriften, München 1926, S. 1-167.

Klebs, ARnOld/ SudHOFF, KARL, Die ersten gedruckten Pestschriften, München 1926.

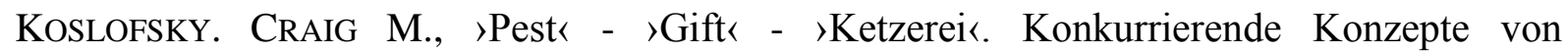
Gemeinschaft und die Verlegung der Friedhöfe (Leipzig 1536), in: JuSSEN BERNHARD/ KOSLOFSKY CRAIG (Hrsg.), Kulturelle Reformation. Sinnformationen in Umbruch 14001600 (Veröffentlichungen des Max-Planck-Instituts für Geschichte, 145), Göttingen 1999. DERS., The reformation of the Dead. Death and Ritual in Early modern Germany, 1450-1700, Wiltshire 2000.

DERS., Die Trennung der Lebenden von den Toten. Friedhofsverlegungen und die Reformation in Leipzig 1536, in: OtTO Gerhard OeXLE (Hrsg.), Memoria als Kultur (Veröffentlichungen des Max-Planck-Instituts für Geschichte, 121), Göttingen 1995, S. 335-385.

KÖRNER, HANS, Der früheste deutsche Einblattholzschnitt (Studia Iconologia 3), Mittenwald 1979. 
Krug, Raphael M., Es ist doch zem Jungsten ein end daran. Die Augsburger Steuerbücher im Spätmittelalter (1346-1430) als Medium städtischer Verwaltung, Augsburg 2006.

KupFERSCHMIDT, HugO, Die Epidemiologie der Pest: der Konzeptwandel in der Erforschung der Infektionsketten seit der Entdeckung des Pesterregers im Jahre 1894, Aarau 1993.

KÜNAST, HANS-JÖRG, <<Getruckt zu Augspurg>>. Buchdruck und Buchhandel in Augsburg zwischen 1468 und 1555, Tübingen 1997.

Labisch, Alfons/ SpREe, ReINHARD, Neuere Entwicklungen und aktuelle Trends in der Sozialgeschichte der Medizin in Deutschland - Rückschau und Ausblick, in: Vierteljahrsschrift für Sozial- und Wirtschaftsgeschichte 84 (1997), S. 171-210; 305-321.

LaChmund, Jens/ Stollberg, GunNar (Hrsg.), The Social Construction of Illness (Medizin. Gesellschaft und Geschichte. Jahrbuch des Instituts für Geschichte der Medizin der Robert Bosch Stiftung, Beiheft 1), Stuttgart 1992.

LANG, MATTHIAS, „Der Vrsprung aber der Pestilentz ist nicht natürlich, sondern übernatürlich...“. Medizinische und theologische Erklärung der Seuche im Spiegel protestantischer Pestschriften 1527-1650, in: OTTO UlBRICHT (Hrsg.), Die leidige Seuche: Pest-Fälle in der Frühen Neuzeit, Köln 2004, S. 133-180.

Le Roy, LAdURIE EMmAnuel, Un concept. L'unification microbienne du monde (XIVeXVIIe siècles), in: Schweizerische Zeitschrift für Geschichte 23 (1973), S. 627-694.

Lengle, Peter, Das »)Gottberatbuch « des Heilig-Geist-Spitals in Augsburg, in: PANKRAZ FRIED (Hrsg.), Bayerisch-schwäbische Landesgeschichte an der Universität Augsburg 1975-1977. Vorträge - Aufsätze - Berichte (Augsburger Beiträge zur Landesgeschichte Bayerisch-Schwabens 1), Sigmaringen 1979, S. 153-162.

Ders., Das Augsburger Heilig-Geist-Spital, in: WALTER PÖtzl (Hrsg.), Herrschaft und Politik. Vom Frühen Mittelater bis zur Gebietsreform (Der Landkreis Augsburg 3), Augsburg 2003, S. 206-215.

Ders., Heilig-Geist-Spital, in: WolfGAng BAER u.a. (Hrsg.), Augsburger Stadtlexikon, Augsburg 1985, S. 156.

DERS., Spitäler, Stiftungen und Bruderschaften, in: GunTHER GotTLieb u.a. (Hrsg.), Geschichte der Stadt Augsburg von der Römerzeit bis in die Gegenwart. Stuttgart 1985, S. 202-208.

LERSCH, BERnHARD MAXIMILIAN, Geschichte der Volksseuchen nach und mit den Berichten der Zeitgenossen, Berlin 1896.

LEVEN, KARL-HEINZ, »At times these ancient facts seem to lie before me like a patient on a hospital bed« - Retrospective Diagnosis and Ancient Medical History, in: HeRMAN F. J. 
Horstmanshoff/ Marten Stol (Hrsg.), Magic and Rationality in Ancient Near Eastern and Graeco-Roman Medicine, Leiden 2004, S. 369-386.

DERS., Die Geschichte der Infektionskrankheiten von der Antike bis ins 20. Jahrhundert, Landsberg/Lech 1997.

DERS., Die „Justinianische“ Pest, in: Jahrbuch des Instituts für Geschichte der Medizin der Robert Bosch Stiftung 6 (1987), S. 137-161.

DERS., Krankheiten - historische Deutung vs. retrospektive Diagnose, in: NORBERT PAUL/ Thomas Schlich (Hrsg.), Medizingeschichte. Aufgaben, Probleme, Perspektiven, Frankfurt am Main/New York 1998, S. 153-185.

DERS., Von Ratten und Menschen - Pest, Geschichte und das Problem der retrospektiven Diagnose, in: Mischa MeIER (Hrsg.), Pest. Die Geschichte eines Menschheitstraumas, Stuttgart 2005, S. 11-32, 377-381.

LeXER, MATthiAS, Mittelhochdeutsches Handwörterbuch: zugleich als Supplement und alphabetischer Index zum Mittelhochdeutschen Wörterbuch von Benecke-MüllerZarnacke, Leipzig 1872-1878.

MachoczeK, Ursula (Bearb.), Deutsche Reichtagsakten unter Kaiser Karl V.. Der Reichstag zu Augsburg 1547/48, 1. Bd. (Deutsche Reichtagsakten. Jüngere Reihe, Bd. 18 herausgegeben von der historischen Kommission bei der Bayerischen Akademie der Wissenschaften durch Eike Wolgast), München 2006.

MARTIN, ERNST (Hrsg.), Hans Folz, Spruch von der Pestilentz, Strassburg 1879.

Martin, Helmut, Die Pest im spätmittelalterlichen Würzburg. Pesterwähnungen in den Quellen. Vom Schwarzen Tod 1348 bis zum Tode Bischof Lorenz' von Bibra 1519, in: Archiv des Historischen Vereins für Unterfranken und Aschaffenburg 117 (1994), S. 239281.

MartZ, Elisabeth, Gesundheitswesen und Ärzte im 16. Jahrhundert, Med. Diss. München 1950.

Mauelshagen, Franz, Pestepidemien im Europa der Frühen Neuzeit (1500-1800), in: Mischa MeIER (Hrsg.), Pest. Die Geschichte eines Menschheitstraumas, Stuttgart 2005, S. 237-265.

MCCORMick, Michael, Rats, Communications, and Plague. Toward an Ecological History, in: Journal of Interdisciplinary History 34 (2003), S. 1-25.

MeIER, Mischa (Hrsg.), Pest. Die Geschichte eines Menschheitstraumas, Stuttgart 2005.

DERS., Die sogenannte Justinianische Pest und ihre Folgen, in: Ders. (Hrsg.), Pest. Die Geschichte eines Menschheitstraumas, Stuttgart 2005, S. 86-107. 
Meyer, Christian G., Tropenmedizin: Infektionskrankheiten, 2. überarb. u. erw. Auflage, Landsberg 2007.

MischlEWSKI, ADALBERT, Das Antoniusfeuer in Mittelalter und früher Neuzeit in Westeuropa, in: Neithard Bulst/ Robert Delort (Hrsg.), Maladies et société (XIIeXVIII siècle), Paris 1989, S. 249-268.

MÜLLER, INGO WILHELM, Humoralmedizin: physiologische, pathologische und therapeutische Grundlagen der galenistischen Heilkunst, Heidelberg 1993.

Müller, Konrad M., Die Pest - Pestheillige, Pestkapellen, Pestsäulen: Von himmlischer Hilfe und in irdischer Not, Wallerstein 2015.

NAPHY, William/ Spicer, AndREw, Der Schwarze Tod. Die Pest in Europa, Essen 2003.

Nockemann, EdiTH, Der Einblattholzschnitt des 15. Jahrhunderts und seine Beziehung zum spätmittelalterlichen Volksleben, Bottrop 1940.

NoHL, Johannes, Der schwarze Tod. Eine Chronik der Pest 1348 bis 1720, Potsdam 1924.

NutTon, Vivian, The Reception of Fracastoro's Theory of Contagion. The Seed That Fell among Thorns? in: Osiris, 2nd series, 1990 (6), S. 196-234.

Oexle, Otto Gerhard, Die Gegenwart der Toten, in: Herman Braet (Hrsg.), Death in the Middle Ages (Mediaevalia Lovaniensia. Series 1. Studia 9), Löwen 1983, S. 19-77.

Pagel, Walter, Das medizinische Weltbild des Paracelsus. Seine Zusammenhänge mit Neuplatonismus und Gnosis (Kosmosophie, Bd. 1), Wiesbaden 1962.

PALMER, RichARD JOHN, The Control of plague in Venice and northern Italy 1348-1600,

Ph.D. thesis, University of Kent at Canterbury 1978.

PATRICK, ADAM, A Consideration of the Nature of the English Sweating Sickness, in: Medical History 9 (1965), S. 272-279.

Penrose, Elisabeth Cartwright, A History of England from the first Invasion by the Romans tot he Present, London 1859.

PietschmanN, Nico, Das Armen- und Krankenwesen im Spätmittelalter und der Frühen Neuzeit: Lebensbedingungen und Lebenswirklichkeit - Die Reichsstädte Augsburg und Nürnberg im Vergleich, in: Zeitschrift des Historischen Vereins für Schwaben, Bd. 107 (2015), S. 151-170.

Pomata, Gianna, Contracting a Cure: Patients, Healers and the Law in Early Modern Bologna, Baltimore/London 1998.

Porzelt, Carolin, Die Pest in Nürnberg. Leben und Herrschen in Pestzeiten in der Reichsstadt Nürnberg (1562-1713), St. Ottilien 2000. 
PÖLNITZ, GöTZ FRH. vON, Anton Fugger, Bd. 1: 1453-1535, (Studien zur Fuggergeschichte, Bd. 13), Tübbingen 1958.

PÜSCHEL, ERICH, Der Englische Schweiß des Jahres 1529 in Deutschland, in: Sudhoffs Archiv 42 (1958), S. 169-181.

RAJKAY, BARBARA, Die Bevölkerungsentwicklung von 1500 bis 1648, in: GUNTHER Gottlieb u.a. (Hrsg.), Geschichte der Stadt Augsburg von der Römerzeit bis in die Gegenwart. Stuttgart 1985, S. 252-258.

Ranger, Terence/ Slack, Paul (Hrsg.), Epidemics and Ideas. Essays on the Historical Perception of Pestilence, Cambridge 1992.

REICKE, SIEGFRIED, Das deutsche Spitalwesen und sein Recht im Mittelalter, Stuttgart 1932 [Nachdruck 1979].

ReSCH, Claudia, »Englischer Schweiß« 1529 in Augsburg: »Suchet man leybsärtzney, warumb sucht man nit ärtzney der seelen?«, in: Medizin, Gesellschaft und Geschichte 28 (2009), S. 97-119.

RiHA, Ortrun, Funktionswandel durch den Kontext: Ortolf-Auszüge als Pesttraktat, in: GuNDOLF KeIL (Hrsg.), "Ein teutsch puech machen": Untersuchungen zur landessprachlichen Vermittlung medizinischen Wissens, Wiesbaden 1993, S. 62-69.

RITZMANN, IRIS, Judenmord als Folge des »Schwarzen Todes«: Ein medizinhistorischer Mythos?, in: Medizin, Geschichte und Gesellschaft 17 (1998), 101-130.

Roberts, Charlotte/ Manchester, Keith, The Archaeology of Disease (3. Ed.), Stroud: Sutton 2005.

Rothschild, Bruce M./ Martin, Larry D., Paleopathology. Disease in the Fossil Record, Boca Raton Fla. 1993.

RodenwaldT, ERnst, Die Gesundheitsgesetzgebung des Magistrato della Sanitá Venedigs: 1486-1550, Heidelberg 1956.

DERS., Pest in Venedig 1575-1577. Ein Beitrag zur Frage der Infektkette bei den Pestepidemien West Europas, in: Sitzungsberichte der Heidelberger Akademie der Wissenschaften. Mathematisch-naturwissenschaftliche Klasse Jahrgang 1952, 2. Abhandlung, Heidelberg 1953, S. 121-381.

RoEcK, BERnd, Eine Stadt in Krieg und Frieden. Studien zur Geschichte der Reichsstadt Augsburg zwischen Kalenderstreit und Parität, 2 Bde. (Schriftenreihe der historischen Kommission bei der bayerischen Akademie der Wissenschaften, Bd. 37), Göttingen 1989. RogGe, JöRG, Für den Gemeinen Nutzen. Politisches Handeln und Politikverständnis von Rat und Bürgerschaft in Augsburg im Spätmittelalter, Tübingen 1996. 
ROTH, FRIEDRICH, Augsburgs Reformationsgeschichte: gekrönte Preisschrift, 4 Bde.: 15171555, München 1901-1911.

RUMMEL, PETER, Ulrich von Augsburg: Bischof, Reichsfürst und Heilliger, Augsburg 1993.

Sachße, Christoph/ Tennstedt, Florian, Geschichte der Armenfürsorge in Deutschland. Vom Mittelalter bis zum 1. Weltkrieg, Stuttgart 1980.

SAlzBrunn, Ingeborg, Das Ende des Augsburger Collegium medicum, in: Bayerisches Ärzteblatt, 28. Jg. Heft 2/73, S. 156-162.

Scheller, Benjamin, Memoria an der Zeitwende. Die Stiftungen Jakob Fuggers des Reichen vor und während der Reformation (ca. 1505-1555), (Stiftungsgeschichten Bd. 3), Berlin 2004.

SCHENDA, Rudolf, Der „gemeine Mann“ und sein medikales Verhalten im 16. und 17. Jahrhundert, in: JOACHIM TElLE (Hrsg.), Pharmazie und der gemeine Mann. Hausarznei und Apotheke in deutschen Schriften der frühen Neuzeit (Ausstellungskataloge Herzog August Bibliothek, 36), Braunschweig 1982, S. 9-20.

Schlich, Thomas, Wissenschaft. Die Herstellung der wissenschaftlichen Fakten als Thema der Geschichtsforschung, in: PAUl Norbert/ ThOMAS Schlich (Hrsg.), Medizingeschichte. Aufgaben, Probleme, Perspektiven, Frankfurt a.M/ New York 1998, S. 107-129.

Schmitt, Wolfram, Regimina, in: Lexikon des Mittelalters, Norbert Angermann u.a. (Hrsg.), Bd. VII, München 1995, Sp. 575-576.

Schmitz-EICHHOF, MARIE Theres, St. Rochus. Ikonographische und medizinhistorische Studien (=Kölner Medizinhistorische Beiträge 3), Köln 1977.

SCHMÖLzER, HILDE, Die Pest in Wien: "Des wütenden todts ein umbständig Beschreibung...", Wien 1985.

SchotTENLOHER, KARL, Doktor Alexander Seitz und seine Schriften. Ein Kleinbild aus dem Münchner Ärzteleben des XVI. Jahrhunderts, München 1925.

SCHÖNER, ERICH, Das Viererschema in der antiken Humoralpathologie, Wiesbaden 1964.

SchÖPPler, HeRmann, Die Geschichte der Pest zu Regensburg, München 1914.

SCHREIBER, WILHELM LudWIG, Pestblätter des 15. Jahrhunderts, Straßburg 1918.

Ders., Handbuch der Holz- und Metallschnitte des XV. Jahrhunderts, 10 Bde. StuttgartNendeln 1969.

SCHREMMER, ECKART, Die Wirtschaftsmetropole Augsburg, in: MAX SPINDLER (Hrsg.), Handbuch der bayerischen Geschichte, Bd. III/2, München 1971, S. 1080-1096. 
Schwalb, ANDrea BIRgit, Das Pariser Gutachten von 1348. Eine Textedition und Interpretation der ersten Summe, Diss. Tübingen 1990.

Schwarz, Klaus, Die Pest in Bremen. Epidemien und freier Handel in einer deutschen Hafenstadt 1350-1713, Bremen 1996.

Shaw, Brent D., Grmek's Pathological Vision, in: Social History of Medicine 4 (1991), S. 329-334.

SEIDA Und LANDSBERG, FrANZ EUGEN vON, Historisch-statistische Beschreibung aller Kirchen-, Schul-, Erziehungs- und Wohlthätigkeitsanstalten in Augsburg. Von ihrem Ursprunge an bis auf die neuesten Zeiten, 2 Bde., Augsburg 1812.

SiEGEL, RudolPh E., Galen's System of Physiology and Medicine, New York 1968.

SiEH, KATARINA, Die Augsburger Stadtverfassung um 1500, in: Zeitschrift des Historischen Vereins für Schwaben und Neuburg 77 (1983), S. 125-149.

SLACK, PAUL, The impact of plague in Tudor and Stuart England, London u.a. 1985.

Sournia, JEAn-Charles, Discipline du diagnostic rétrospectiv, in: NeITHARD Bulst/ Robert Delort (Hrsg.): Maladies et société (XIIe-XVIII siècle), Paris 1989, S. 57-64.

Stein, Claudia, Die Behandlung der Franzosenkrankheit in der Frühen Neuzeit am Beispiel Augsburg, Stuttgart 2003.

Sticker, GEORG, Abhandlungen aus der Seuchengeschichte und Seuchenlehre, Bd. 1: Die Pest. Teil 1 und 2: Die Pest als Seuche und als Plage, Gießen 1908-1910

StRASSER, Gerhard F., Ansteckungstheorien der Pest in der Frühen Neuzeit am Beispiel von Girolamo Fracastoro und Athansius Kircher, in: Petra FeuersteIN-Herz (Hrsg.), Gotts verhengnis und seine straffe - zur Geschichte der Seuchen in der Frühen Neuzeit, (Ausstellungskataloge der Herzog August Bibliothek Nr. 84), Braunschweig 2005, S. 6976.

SUDHOFF, KARL, Deutsche medizinische Inkunabeln. Bibliographisch-literarische Untersuchungen, Leipzig 1908.

DERS., Ein neues deutsches Pestblatt, gedruckt zu Augsburg ca. 1483 bei Hermann Kästlin, in: Archiv für Geschichte der Medizin 3 (1910), S. 63-66.

DERS., Pestschriften aus den ersten 150 Jahren nach der Epidemie des „schwarzen Todes“ 1348, Archiv für Geschichte der Medizin, 1911-1925 Leipzig.

DERS., Der Ulmer Stadtarzt Dr. Heinrich Steinhöwel (1420-1482) als Pestautor, in: Ders./ ARNOLD KLEBS, Die ersten gedruckten Pestschriften, München 1926, S. 169-211ff. 
Telle, JoAChim (Hrsg.), Pharmazie und der gemeine Mann. Hausarznei und Apotheke in deutschen Schriften der frühen Neuzeit (Ausstellungskataloge Herzog August Bibliothek, 36), Braunschweig 1982.

DERS., Wolfgang Talhauser. Zu Leben und Werk eines Augsburger Stadtarztes und seinen Beziehungen zu Paracelsus und Schwenkfeld, in: Medizinhistorisches Journal 7 (1972), S. $1-30$.

Trometer, Johann, Das Augsburger Pilgerhaus, Diss. Augsburg 1997.

TRÜB, PAUL, Heilige und Krankheit, Stuttgart 1987.

Thwaites, Guy/ Taviner, Mark/ Gant, Vanya, The English Sweating Sickness, 14851551, in: New England Journal of Medicine 336 (1997), S. 580-582.

Twigg, Graham I., The Black Death in England. An Epidemiological Dilemma, in: Neithard Bulst/ Robert Delort (Hrsg.), Maladies et société (XIIe-XVIII siècle), Paris 1989, S. 75-98.

Ulbricht, OtTo (Hrsg.), Die leidige Seuche: Pest-Fälle in der Frühen Neuzeit, Köln 2004.

DERS., Pesthospitäler in deutschsprachigen Gebieten in der Frühen Neuzeit. Gründung, Wirkung und Wahrnehmung, in: DeRs. (Hrsg.), Die leidige Seuche: Pest-Fälle in der Frühen Neuzeit, Köln 2004, S. 96-132.

Wahrmann, Carl Christian/ Buchsteiner, Martin/ Strahl, AntJe (Hrsg.), Seuche und Mensch: Herausforderung in den Jahrhunderten. Ergebnisse der internationalen Tagung vom 29.-31. Oktober 2010 in Rostock (Historische Forschungen Bd. 95), Berlin 2012.

Warmbrunn, PAul, Zwei Konfessionen in einer Stadt: das Zusammenleben von Katholiken und Protestanten in den paritätischen Reichsstädten Augsburg, Biberach, Ravensburg und Dinkelsbühl von 1548 bis 1648, Wiesbaden 1983.

Watson, Gilbert, Theriac and Mithridatium: A study in therapeutics (= Publications of the Wellcome Historical medical library, Neue Folge, 9), London 1966.

WEBER, DIETER, Geschichtsschreibung in Augsburg. Hektor Müllich und die reichsstädtische Chronistik des Spätmittelalters, Würzburg 1984.

Weeks, ANDREw, Paracelsus. Speculative Theory and the Crisis of Early Modern Reformation, Albany 1997.

WERNER, ANTON, Die örtlichen Stiftungen für die Zwecke des Unterrichts und der Wohltätigkeit in der Stadt Augsburg: Historisch und systematisch dargestellt, Augsburg 1899.

WinCKELMANN, OTTO, Über die ältesten Armenordnungen der Reformationszeit (1522-1525), in: Historische Vierteljahresschrift 17 (1914/15), S. 187-228, 361-400. 
WINKLER, LuTZ, Galens Schrift 'De antidotis': ein Beitrag zur Geschichte von Antidot und Theriak, Marburg 1980.

WoEHLKENS, ERICH, Pest und Ruhr im 16. und 17. Jahrhundert. Grundlagen einer statistischtopographischen Beschreibung der großen Seuchen, insbesondere in der Stadt Uelzen, Uelzen 1954.

Wright, Peter/ Treacher, Andrew, The Problem of Medical Knowledge. Examing the Social Construction of Medicin, Edinburgh 1982.

Wüst, Wolfgang, Armut und Besitz, Frömmigkeit und Fürsorge. Spitäler in Mittelater und Frühen Neuzeit. Die Zucht- und Policeyordnung des Heilig-Geist-Spitals in Augsburg von 1764, in: Zeitschrift des Historischen Vereins für Schwaben, Bd. 108 (2016), S. 185-234.

ZELler, Michael, Rochus. Die Pest und ihr Patron (Nürnberger Schriften 2), Nürnberg 1989.

ZINN, KaRL GeORG, Kanonen und Pest. Über die Ursprünge der Neuzeit im 14. Und 15. Jahrhundert, Opladen 1989.

ZOEPFL, FRIEDRICH, Das Bistum Augsburg und seine Bischöfe im Mittelalter, Augsburg 1955.

DERS., Das Bistum Augsburg und seine Bischöfe im Reformationsjahrhundert, Augsburg 1969.

ZoRn, WolfGang, Augsburg. Geschichte einer deutschen Stadt, Augsburg 1972. 